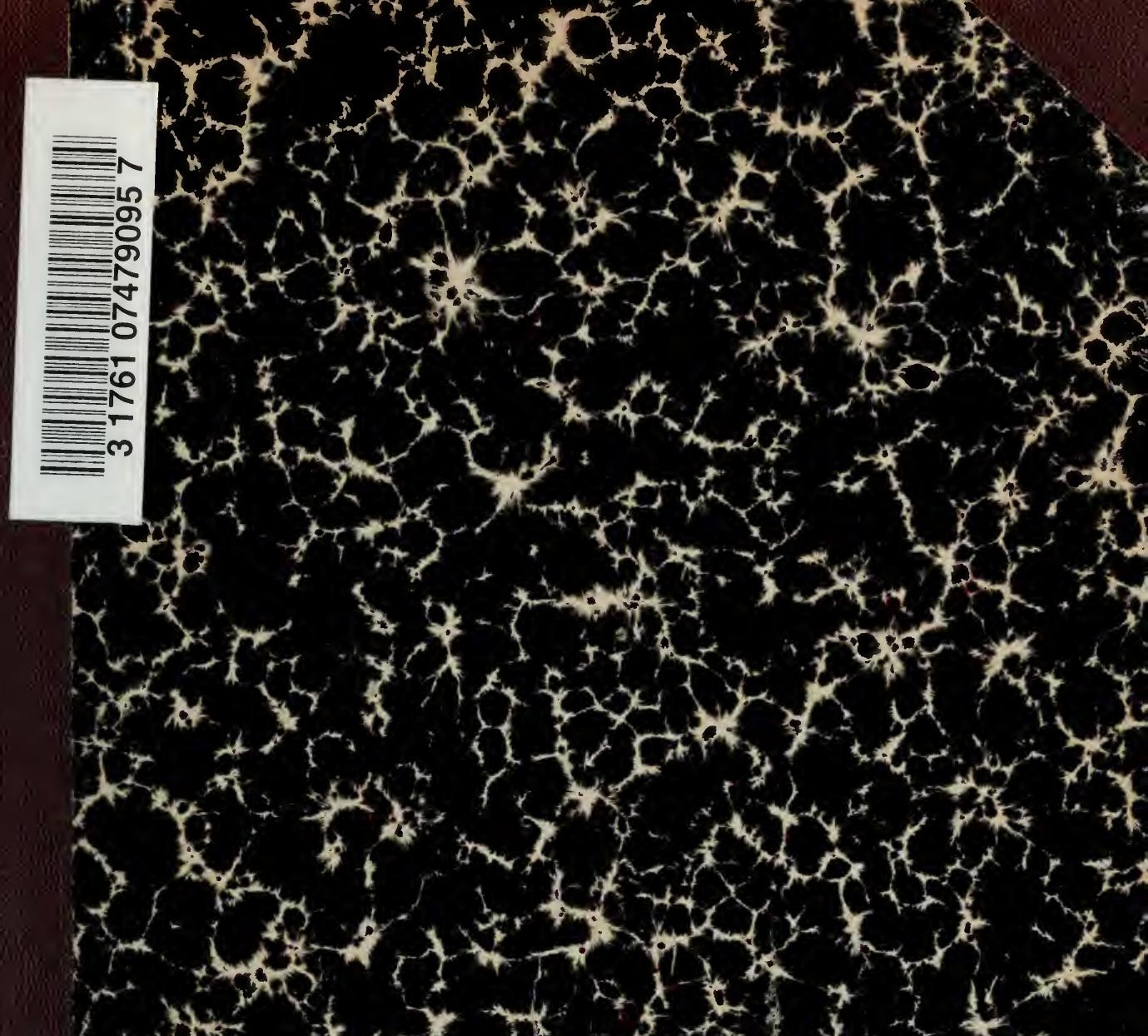

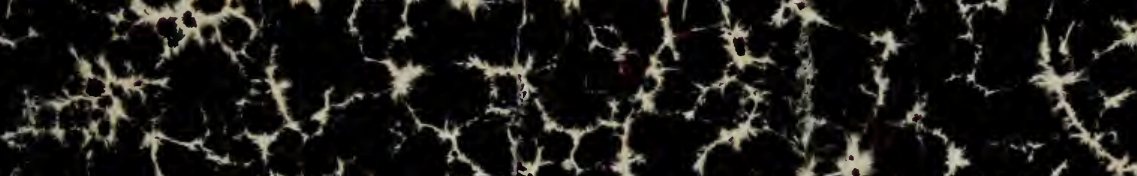

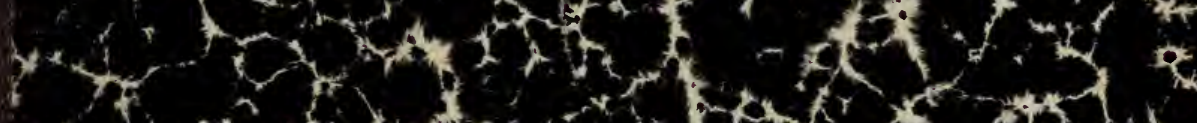
(

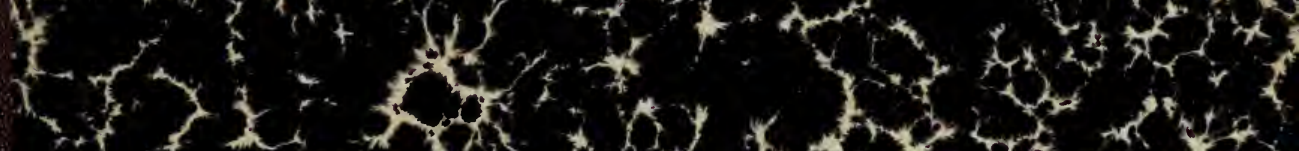

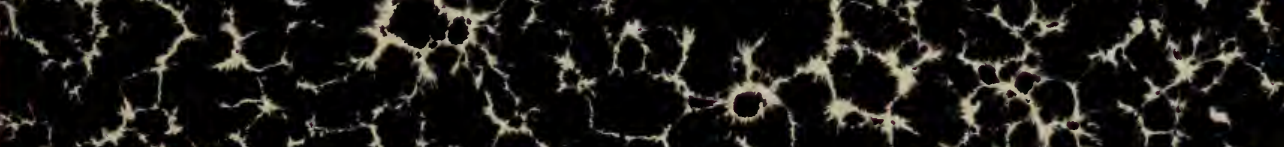

$\mathrm{GCY}_{274}+4+2+4$

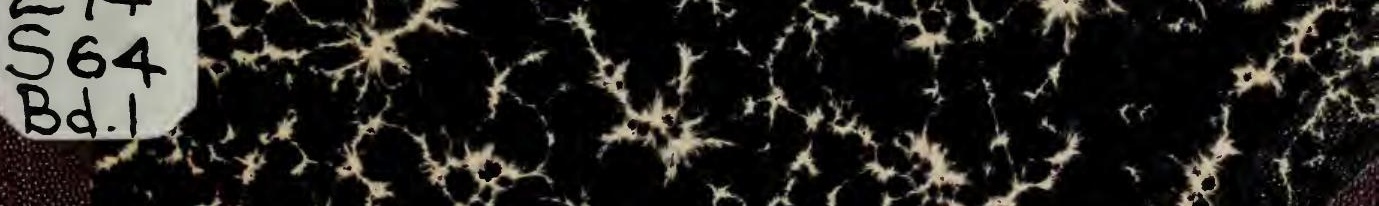
$x^{2}+2$ 


Digitized by the Internet Archive in 2010 with funding from University of Toronto 


\title{
Studien zur Ozeanographie der südwestlichen Ostsee
}

\author{
I.
}

Von

Dr. Hans Spethmann

Privatdozent für Geographie an der Universität Berlin

Mit 18 Textfiguren

$$
\frac{568.9814}{16.9 .53}
$$

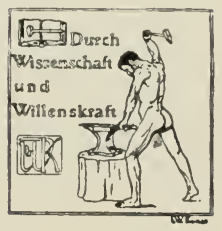

Leipzig 1913

Verlag von Dr. Werner Klinkhardt 
Ge

2.74

564

pd. 1 


\section{VORWORT.}

Für die Beltsee und den Öresund liegt ein ungemein reiches ozeanographisches Tatsachenmaterial vor. Seit Jahren sind in diesem Gebiet seitens Dänemark hydrographische Messungen in dichter Reihenfolge systematisch rorgenommen. Von 1902 ab haben sie durch die Fahrten der "Internationalen Meeresforschung" eine bedeutsame Erweiterung erfahren. Eine größere Anzahl isoliert stehender Forschungen bietet eine willkommene Ergänzung.

Den mannigfachen Beobachtungen ist für unser Gebiet bereits von zwei Seiten, von Jacobsen und Ruppin näher getreten worden ${ }^{1}$ ), so daß es bedenklich erscheinen möchte, wenn noch ein Dritter zu dem gleichen Gegenstand das Wort ergreift. Indessen, Jacobsen konnte 1908 für seinen Zweck nur dänische Beobachtungen bringen, Ruppin verwertet vier Jahre später neben den Messungen auf den dänischen Feuerschiffen nur deutsche Beobachtungen. Bei dem innigen Durcheinander der Lage der einzelnen Stationen erscheint es mir hingegen das Gegebene, alle Beobachtungen beider Länder gleichzeitig zu berücksichtigen, wenn man die Hydrographie der Beltsee und des Sundes aufklären will.-Jacobșęn fäat sich streng an denselben Zeitraum, an die Jahre 1880 bis 1907, Ruppin nimmt bald Material von 1902 bis 1907, bald von 1902 bis 1911, ohne genaueres darüber mitzuteilen oder die Werte einander auszugleichen. Die Resultate beider Autoren sind also nicht miteinander vergleichbar. Drittens bezweckt Jacobsen nur Mittelwerte zu liefern. Ruppin will einen „Rückblick über die gewønnenen Tatsachen" geben, wobei er gleichfalls in erster Linie Mittelwerte berücksichtigt und von ihren Ergebnissen ausgeht. Meines Erachtens ist

$\left.{ }^{1}\right)$ I. P. Jacobsen, Mittelwerte von Temperatur und Salzgehalt, bearbeitet nach hydrographischen Beobachtungen in dänischen Gewässern 1880-1907. Meddelelser fra Kommissionen for Havundersögelse, Serie Hydrografi, Bd. I, Kopenhagen 1908 und E. Ruppin, Beiträge zur Hydrographie der Belt- und Ostsee. Wissenschaftl. Meeresuntersuchungen, N. F., Bd. XIV, Abteilung Kiel. 1912. 
es bei der auBerordentlich starken Verwicklung der hydrographischen Verhältnisse der Beltsee und des sundes ratsamer, von Einzelphänomenen aus zu Mittelwerten fortzuschreiten, orler, sobald dieses nicht angängig ist, möglichst viele Einzelphänomene in den Kreis der Diskussion zu ziehen. Für unbedingt notwendig aber erachte ich es bei den großen Schwankungen von Jahr zu Jahr, eine einheitliche Periode zugrunde \%u legen, für die nur die Zeit seit Norember 1902 in Frage kommt, da seit dem Beginn der .Internationalen Meeresforschung" auch die Messungen auf den dänischen Feuerschiffen nach den gleichen Methoden und Grundsätzen organisiert wurden. Eine von diesen Gesichtspunkten vorgenommene neue Bearbeitung ozeanographischer Fragen in der südwestlichen Ostsee hat deshalb in wichtigen Punkten ron Ruppin abweichende Ergebnisse gezeitigt. Ich werde diese im einzelnen in den folgenden Ausführungen nicht jeweils hervorkehren, sondern in einem späteren Abschnitt über die Entwicklung der ozeanographischen Kenntnisse der südwestlichen Ostsee im Zusammenhang dartun.

Der verarbeitete Beobachtungsschatz ist von mir nur zu einem verschwindend kleinen Bruchteil selber gewonnen worden. Auf zwei Fahrten mit dem Forschungsdampfer "Poseidon" zwischen Bornholm und Alsen und auf einer in das Kattegat, Skagerak und in die Nordsee lernte ich 1909 den ozeanographischen und meteorologischen Dienst versehen, und als Hilfsassistent im ozeanographischen Laboratorium in Kiel während des Winters 1909/10 wurde mir die Art der Behandlung der Messungen bekannt. Da die Messungen und ihre Auswertung auf dem "Poseidon“" wie in Kiel, dank der Organisation der "Internationalen Meeresforschung“, sich in allen wichtigen Punkten nach denselben Verfahren vollziehen, wie sie auf den Fahrten der anderen Staaten unseres Gebietes angewandt werden, so glaube ich auch einen Einblick in die Beschaffung des übrigen, im folgenden benutzten Materials gewonnen zu haben.

Spiekeroog, den 16. September 1913.

Hans Spethmann. 


\section{Inhaltsverzeichnis.}

Einfiilhrung

Die Tiefencerhältnisse

Die Verteilung der Tiefen: Sund, Samsö Belt und Großer Belt, Stammbelt, Kleiner Belt S. 3. - Morphometrische Werte S. 7. - Die Tiefenverhältnisse des südlichen Kattegat und der Arkonasee S. 8.

Die Gewinnung der täglichen Beobachtungen . . . . . . . . . . . . . .

Die Lage der Feuerschiffe Schultz Grund, Gjedser Rev, Drogden und Lappe Grund S. 10. - Die Vornahme der Beobachtungen S. 11. - Die Genauigkeit der Beobachtungen S. 12.

Sturm und Strom

Wind und Strom S. 13. - Die angewandte Methode S. 14. - Drogden S. 16. - Schultz Grund S. 18. - Lappe Grund S. 18. - Giedser Rev S. 19.-Gesamtbild für die vier Feuerschiffe S. 20. - Die Stromstärke S. 22. - Der hydrographische Wert S. 23. - Tabellen S. 24. Auslauf und Einlauf

Nethodisches S. 32. - Die IIonatsmittel S. 33. - Die Jahresmittel S. 34. - Die Wasserstandsschwankungen S. 35. - Die Stromgeschwindigkeit S. 37. - Der Wasseraustausch durch Sund und Stammbelt S. 39. Tabellen S. 40.

Schwankiungen des Salzgehaltes I

Drogden S. 44. - Lappe Grund S. 50. - Schultz Grund S. 52. -

Gjedser Rev. S. 54. - Fehmarnbelt S. 56. - Die monatlichen und die jährlichen Schwankungen S. 56. - Die Ursache der Schwankungen S. 59. Synoptisches Bild für die Schwankungen an den vier Feuerschiffen S.61. Tabellen 63.

Schwankungen des Salzgehaltes II.

Die Gewinnung der Beobachtungen S. 72. - Die Genauigkeit der Beobachtungen S. 74. - Der Gang des Salzgehaltes vom südlichen Kattegat zur Arkonasee S. 75. - Die Extreme am Boden S. 80. - Das atlantische Wasser S. 82. - Die Extreme an der Oberfläche S. 8t. - Die Schwankungen zwischen Boden und Oberfläche S. 85. - Tabellen S. 86.

Synoptisches Bild der Salzgehaltsverteilung. . . . . . . . . . . . . . .
Die Arkonasee S. 92. - Die Funktionen der Kadetrinne und des Sundes

S. 95. - Der Stammbelt S. 96. - Die Kieler Bucht S. 98. - Der Große Belt und der Samsö Belt S. 99. - Das südliche Kattegat S. 100. - Tabellen S. 102 . 



\section{Einführung.}

Aus dem Becken des Atlantischen Ozeans zweigt an der Westküste Skandinaviens die Norwegische Rinne nach Süden ab. Mit Tiefen über $200 \mathrm{~m}$ folgt sie dem Umriß der Halbinsel bis ins Skagerrak, und mit solchen bis zu $100 \mathrm{~m}$ lassen sich ihre Ausläufer bis zur Höhe von Anholt im Kattegat leicht erkennen. Dann aber verliert sie sich, der Boden des Meeres bleibt in Steigung, und bis zu $-18 \mathrm{~m}$ müssen wir nach manchem Auf und Ab hinan, wenn wir in die große Hohlform gelangen wollen, die mit Wasser erfüllt in den Norden unseres Kontinents bis nach Haparanda und St. Petersburg hineingreift. Zwischen Falster und Darsser Ort liegt dieser wichtige Punkt des höchsten Durchlasses, von dem wir langsam wieder hinabgelangen, bis zu $50 \mathrm{~m}$ nördlich Arkona, bis zu $100 \mathrm{~m}$ auf der Höhe von Memel und bis zu etwas mehr denn $460 \mathrm{~m}$ am tiefsten Punkt, dem Landsort Tief zwischen Gotland und der Einfahrt nach Stockholm.

Die Schwelle, die wir durchmessen haben, ist ein Unterteil einer größeren Schwelle, die die südwestliche Ostsee durchzieht und die vom Nordende der dänischen Inseln bis zur südlich gelegenen deutschen Küste reicht. Deutlich stoßen an sie im Norden und im Osten Tiefen bis zu $50 \mathrm{~m}$ und mehr, sie selbst aber bleibt überall über $30 \mathrm{~m}$, wenn man von einigen isolierten, noch nicht $4 \mathrm{qkm}$ großen Stellen von 50 bis $81 \mathrm{~m}$ Tiefe absieht. Über diese große Schwelle muß alles Wasser fließen, das aus dem Innern der Ostsee zum Ozean will oder sich in umgekehrter Richtung bewegt. Gleichzeitig erfährt es noch einen anderen Zwang; seine Ausbreitungsfläche engt sich beträchtlich ein. Nur zwei schmale Straßen vermitteln ihm ein Durchfluten, der Öresund und jene namenlose Straße, die zwischen Rügen und Möen beginnt und sich bis zum Fehrmarnbelt hinzieht, westlich dessen sie sich in die beiden Arme des Großen und Kleinen Beltes gabelt. 
Die doppelte Komplikation der Verminderung der Tiefe und der Einengung des Raumes an dem änßeren Endo eines Binnenmeeres gibt diesem Gebiete seine ozeanographische Stellung. Hier muß sich der Konflikt zwischen dem auslaufenden Süßwasser und dem einlaufenden Salzwasser schon aus morphologischen Gründen mit besonderer Heftigkeit entwickeln. Er wird noch verschärft durch einen klimatischen Faktor. Die reichliche Zufuhr an Süßwasser, die die Ostseo alljährlich empfängt und in den Ozein ergießt, erzengt an der Oberfläche einen auslaufenden Strom. Er muß sich nach westlichen Richtungen bewegren, entgegengesetzt zu den vorwaltenden Winden, die ihn nach Osten zu treiben sich bemühen.

Der Schauplatz dieser Kämpfe deckt sich ungefähr mit dem Begriff Sund und Beltsee. Als Krümmel den letzten der beiden Namen 189; aufstellte, verband er mit ihm das Kattegat, den Oresund und die Belte bis zur Darsser Schwelle zwischen Darsser Ort und Falster. Auf Beschluß des Zentralausschusses für die Internationale Erforschung der nordeuropäischen Meere wurde in Einvernehmen mit Kr ümmel der Begriff in seiner Ausdehnung beschränkt, Sund und Kattegat wurden nicht hineingezogen, so daß er nur das eigentliche Gebiet der Belte umschließt, Samsö, Großen und Langelandbelt, Kleinen und Alsenbelt, Kieler Bucht, Fehmarn Belt, Mecklenburger Bucht nebst Kadetrinne. Im Interesse internationaler Verständlichkeit wollen wir diese Cmgrenzung beibehalten, obwohl uns die erste Fassung Krümmels ohne Kattegat weit treffender $\mathrm{zu}$ sein scheint.

Die Schwelle, die wir hinsichtlich ozeanographischer Wirkungen behandeln, setzt sich also aus Beltsee und Sund, unter dem stets der Öresund gemeint ist, zusammen. Im Norden stößt sie an das Kattegat, im Osten an das, was vielfach "Ostsee im eigentlichen Sinne" genannt wird. Ich habe an anderer Stelle schon auf die Schwierigkeiten aufmerksam gemacht ${ }^{1}$ ), die sich durch die doppelte Verwendung des Namens „Ostsee“ ergeben, einmal für die gesainte Wasserfläche von SkagenNarstrand bis zu den Innenenden des Bottnischen und Finnischen Busens, dann für die Fläche von den Ålandsinseln bis zur Beltsee und zum Sund. Für diese engere Begrenzung habe ich das Wort „Gotlandsee" vorgeschlagen, da ungefähr in ihrer Mitte die große Insel Gotland gelegen ist und da das Gotlandbecken, das im Süden bis auf die Höhe von Memel reicht, ihr das ozeanographische und morpholo-

$\left.{ }^{1}\right)$ H. Spethmann, Der Wasserhaushalt der Ostsee. Zeitschr. Ges. f. Erdkunde, Berlin 1912. 
gische Gepräge aufdrückt. In Westen wollen wir sie bei Bornholın begrenzen, da das Wasserbecken zwischen Vorpommern, Bornholm, Schonen, Seeland, Möen und Falster, das in der Hauptsache von dem Arkonabecken eingenommen wird und im folgenden "Arkonasee" genannt wird, einen besonderen Charakter trägt. Es ist das vermittelnde Glied zwischen den Tiefen der Ostsee und der Überlaufschwelle, zwischen den Gebieten der Ausbildung konstanter Zustände und fortwährenden Mischens. Wir unterscheiden demnach außer der Beltsee und dem Sund das Kattegat, die Arkonasee und die Gotlandsee.

Der genaue Umriß der Beltsee, der für die morphometrische Auswertung von Belang wird, ist folgender: Die Verbindungslinie zwischen der Nordspitze von Seelands Odde, Schultz Grund Feuerschiff, der Nordspitze der Insel Helm und Hassenöre dient als Scheide gegen das Kattegat. Zwischen Falster und Möen wird eine schmale Stelle am Grön Sund benutzt, zwischen Möen und Seeland eine Linie von Ulvshale nach der Ostspitze von Jungshoved. Schwierig ist die lineare Eintragung der Darsser Schwelle. Ein vorspringendes Kap bei Ulslev an der Ostküste von Falster wie der Schnittpunkt von 12 Grad 30' O. L. mit der Westküste des Darss, der nahe Darsser Ort liegt und wegen des ungemein raschen Wanderns dieser Spitze besser geeignet ist, geben die Landmarken für einen Kreisbogen ab, der so gelegt ist, da B die Kadetrinne ganz der Beltsee zufällt. Den Sund begrenzt im Norden die Verbindungslinie zwischen der Nordwestspitze Kullens und Gilleleje, im Süden eine solche von Falsterbo nach dem östlichsten Punkte von Stevns Klint auf Seeland.

\section{Die Tiefenverhältnisse.}

Die Verteilung der Tiefen: Sund, Samső Belt und Großer Belt, Stammbelt, Kleiner Belt S. 3. - Morphometrische Werte S. 7. - Die Tiefenverhältnisse des südlichen Kattegat und der Arkonasee S. S.

Der morphologische Grundzug der Beltsee und des Sundes ist die Entwicklung von Rinnen. So viele Inseln, Engen und Untiefen sich dem Wasser auch hindernd in den Weg stellen, so ergeben sich doch ungezwungen mehrere Hauptrinnen, die als Kleiner Belt, Großer Belt und Sund schon seit langem unterschieden werden. Die flachste Rinne unter ihnen ist der Sund. Er stellt eine ron einem Scheitel sich sanft nach zwei Seiten neigende Rinne dar. Der Scheitel ist südlich Salt- 
holm gelegen in Gestalt zweier Uberlaufschwellen, den Drogden und der Flintrinne. Die erste ist dio flachste unter ihnen, nach den Seckiarten erreicht sie nur 6,9 m Tiefe, während dio andere bis $10,5 \mathrm{~m}$ hinabgeht. Die Rinnen laufen mit Tiefen unter $10 \mathrm{~m}$ westlich und östlich um Saltholm derart herum, daß die Irogden mit der Fortsetzung des Holländer Tiefs den kürzesten Weg nach Norden abgeben und auch orographisch am besten entwickelt sind. Nördlich des Saltholm Flachs vereinigen sich beide, un östlich von Hven auf die Enge HelsingörHelsingborg zu halten, von der aus sie sich trichterförmig ins Kattegat öfnen, ohne irgendeine Schwelle zu überschreiten. Wohl treten dagegen im Sund kleine Schwellen in der Rinne auf, die jedoch nicht gestatten, größere Becken abzugliedern. Es sind zwischen Rücken Vertiefungen um 10 bis $20 \mathrm{~m}$, die zum Teil an Stromengen liegen. Südlich der beiden Überlaufschwellen ist kein schärferes Bodenrelief vorhanden, es fällt ziemlich gleichmäBig auf 40 bis $50 \mathrm{~m}$ ins Arkonabecken hinab.

Samsö Belt und Großer Belt besitzen an ihrem Boden eine sie einheitlich verbindende Rinne und würden als ein Ganzes angesehen werden dürfen, wenn nicht südlich der Insel Samsö eine Verbindung zum Kleinen Belt abzweigt, die einen sehr hohen Rücken überschreiten muß, der den allgemeinen Tiefenverhältnissen nach beurteilt die Rolle einer hydrographischen Scheide spielt. Der Samsö Belt ist eine wenig einheitlich gegliederte Wasserfläche zwischen der Sejröbucht, der Insel Samsö und Yder Flach. Im Relief wird sie durch eine ausgesprochene Rinne zusammengehalten, die wie die schon im Sund besprochenen Rinnen und überhaupt rlle Rinnen dieses Gebietes sich aus einer Anzahl von Rücken und Becken zusammenfügt. Sie bleibt stets unter $30 \mathrm{~m}$ und geht stellenweise bis über $55 \mathrm{~m}$ hinab. Eine flache Schwelle mit nur $33 \mathrm{~m}$ Maximalhöhe trennt sie von ihrer Fortsetzung in der breiten, talartigen Wasserfläche des Großen Beltes, in welchen sie sich halbwegs bis nach Sprogö verliert. Auf der Höhe dieser Insel setzt eine neue ein, die sich vielfach bis über $40 \mathrm{~m}$ tief ununterbrochen bis zum Südende des Großen Beltes fortsetzt, ohne eine schärfere Gliederung zu besitzen. Zwischen den beiden Rinnen liegt ein breiter und flacher Sattel mit einem tiefsten Úberlauf von $28 \mathrm{~m}$, der im GroBen Belt einen kleinen Nordteil von einem größeren Südteil scheidet. Von dem letzteren zweigen sich zwischen Seeland und Lolland die Eingänge zum Smaaland Fahrwasser ab, das jedoch hauptsächlich durch den Omösund und Agersösund mit dem Großen Belt verbunden ist. Außerdem besitzt das Smaaland Fahrwasser einen direkten Ausgang in die Arkonasee durch 
den Stor Strom und Grön Sund zwischen Falster einerseits und Seeland und Möen anderseits, der vielfach, aber nicht überall, von einer 10-20 m tiefen Rinne durchfurcht ist, während der Guldborg Sund zwischen Falster und Lolland nicht $10 \mathrm{~m}$ erreicht.

Dieser führt in das Stück Beltsee zwischen Darsser Schwelle und einer Schwelle zwischen Fehmarn und der Südspitze von Langeland, die nur südlich des Gulstav Flach unterbrochen ist. Es ist jenes wichtige Stück, das die beiden Wege des Großen und Kleinen Beltes vereint, wie ein Stamm seine İste, und zur Arkonasee führt; wir wollen es kurz als „Stammbelt" bezeichnen. Der talartige Charakter verliert sich in ihm, der Enge des Fehmarnbeltes geht die geräumige Kieler Bucht voraus und folgt die ebenfalls breite Mecklenburger Bucht. Die Tiefen bleiben unter $20 \mathrm{~m}$, fallen aber nur im Vinds Grar nordwestlich Fehmarn unter $30 \mathrm{~m}$. Erst südlich Gjedser stellen sich Tiefen über $20 \mathrm{~m}$ ein, die aber westlich der Kadetrinne keine so ausgesprochene Schwelle formen wie nördlich von ihr die Darsser Schwelle mit $18 \mathrm{~m}$ maximaler Tiefe.

Die zweite ZufuhrstraBe des Stammbeltes, der Kleine Belt mit seiner Fortsetzung durch den Norden der Kieler Bucht, zerfällt in zwei Teile, die beide talartige Züge tragen und die sich lediglich durch ihre Breite und Tiefe voneinander trennen. Der südliche, bis Aarö reichend, ist vergleichsweise breit und bleibt mit Ausnahme einer Unsicherheit bein Alsenstein durchweg unter $30 \mathrm{~m}$, eine Tiefe, die bis zur FehmarnLangeland Schwelle auf $-20 \mathrm{~m}$ abnimmt. Der nördliche enge Teil ist dagegen vielfach über $20 \mathrm{~m}$ und besitzt eine Schwelle bei der Insel Aarö mit 14 m Maximaltiefe. Weiter nordwärts erreicht er aber vom Bredning bis fast zum Tragten durchgehends Tiefen bis $40 \mathrm{~m}$ und an einer Stelle bei der Insel Fänö die größte Tiefe der Beltsee mit $81 \mathrm{~m}$.

Fassen wir das Übergangsgebiet zwischen der Arkonasee und dem Kattegat zusammen, so ergeben sich die beiden Straßen des Sundes und des Stammbeltes, von denen die letztere sich in den Großen und Kleinen Belt teilt. Der Sund besitzt seine tiefste Úberlaufschwelle in der Flintrinne, der Stammbelt in der Darsser Schwelle, der Große Belt am Südeingang, der Kleine Belt nicht weit vom Nordausgang. Diese Schwellen spielen naturgemäß eine wichtige ozeanographische Bedeutung. Wir haben deshalb ihre Querschnitte auf Grund der Seekarten berechnet und in der umseitig stehenden Tabelle vereinigt.

Die Grenzen sind folgendermaßen gezogen worden. Für den Kleinen Belt haben wir auf der deutschen Seekarte 330 (Tit. II, Nr.5) von 1907 
von Orbyhage an nach der Südostspitze der kleinen Insel Linderum gemessen, von dort nach der Nordbake auf Aarö und in gerader Linie über Bastholı hinweg zum leuer auf Baagö. Von Böstende führt der Schnitt in der Richtung auf die Kirche von Husby über den Baagösund. Die Schwelle nördlich Sprogö ist gerechnet vom Fischleuer auf Reersö nach der Nordküste von Musholm so, daß der westlichste Punkt des Hafens von Musholm angepeilt wurde. Die Südwestecke der Insel in Verbindung mit den Skalkenbjerg lieferte das Profil über die Hauptstraße des Großen Beltes. Am Südausgang der Wasserstraße wurde vom Feuer Kjels Noor bei Fakkebjerg auf die Kirche von Kappel gehalten. Beide Querschnitte wurden auf der deutschen Seekarte 46 (Tit. II. Nr. 9) genommen. Die Darsser Schwelle wurde gemäß der schon angegebenen L'mgrenzung vermessen. Die Schwellen des Sundes wurden in ihren beiden Strecken östlich und westlich Saltholm berechnet. Für Oscars Grund in der Flintrinne wurde auf der deutschen Seekarte 246 (Tit. II, Nr. 16) die Richtung der Verbindungslinie Feuerschiff Oscars Grund und Glocken Tonne bei Söndre Flint gewählt, für Drogden die Verbindung der Bojen bei Holmetunge und Viderdalskrog, von der ersten wurde zur Südspitze von Saltholm weitergezogen, von der andern genau östlich bis zur Küste von Seeland.

Die Messungen ergaben:

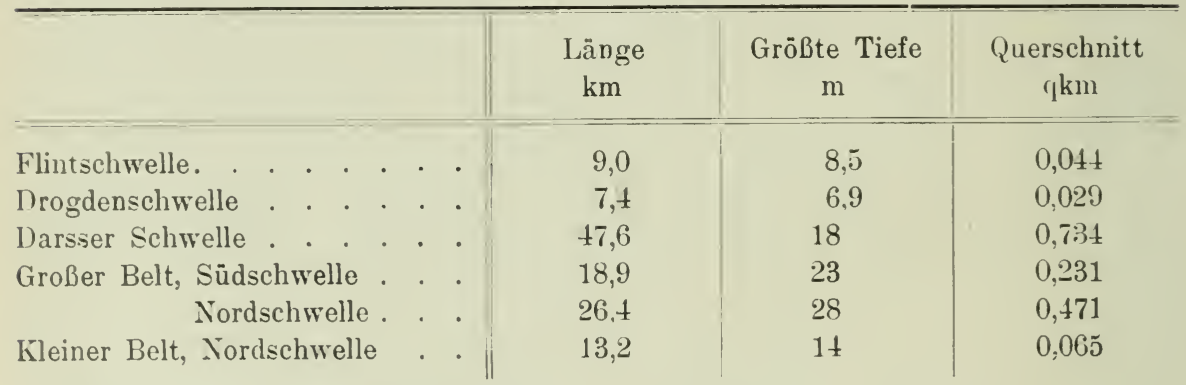

Wir sehen, welche bedeutende Rolle die Darsser Schwelle und die Sundschwelle mit ihren geringen Tiefen spielen müssen, während die Schwelle im nördlichen Kleinen Belt durch die niedrigere Lage der beiden des Großen Beltes ziemlich außer Funktion bei der Kommunikation zwischen Kattegat und Arkona gesetzt wird. Die Sundschwellen zeichnen sich ferner noch durch ein geringes Querschnittsareal aus, das rund zehnmal kleiner ist als das der Darsser Schwelle.

Außerdem haben wir auch die Querschnitte für die Wasserflächen bei vier Leuchtschiffen, die uns wegen der auf ihnen angestellten Be- 
obachtungen noch beschäftigen werden, zusammengestellt, und zwar wurden von ihnen aus die beiderseitigen landnächsten Punkte für die Querschnitte gewählt. Die Lage der Schnitte war bei Schultz GrundFeuerschiff im Norden des Samsöbeltes die dortige Begrenzung der Beltsee, bei Lappe Grund die Richtung vom Schloß bei Stubbarp auf der schwedischen Seite mit dem Feuerschiff, bei Gjedser Rev die Verbindung von Gjedser Odde mit dem Bakelberg des Mecklenburgischen Fischlandes, bei Drogden jene von Vigerdalskrog auf Amager mit dem Feuerschiff, dann die Kirche ron Bunkerflo auf Schonen. Es ergeben sich folgende Werte:

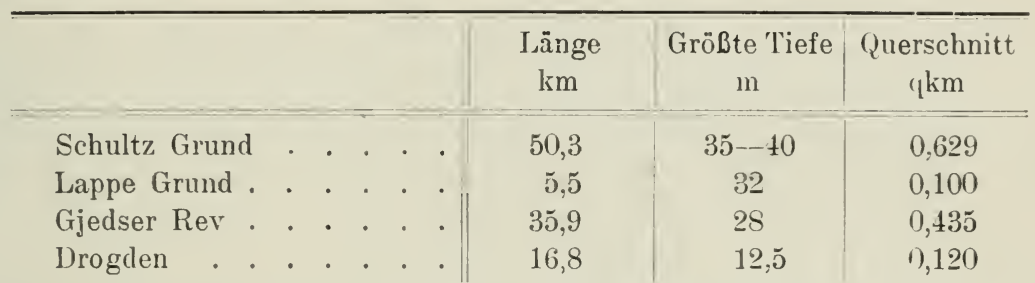

Auch hier fällt beim Sund das geringe Maß der DurchlaBöffnung auf. Das Relief der Schwellen ist wie das der ganzen Beltsee flach geböscht. Beträgt doch die größte Tiefe nur $81 \mathrm{~m}$, sie ist bei der Insel Fänö im nördlichen Kleinen Belt gelegen. Die beiden nächstgrößeren Tiefen finden sich in der Nähe, zwei Stellen mit $69 \mathrm{~m}$. Alle drei Orte sind nur kleine kesselartige Löcher von geringer Ausdehnung, überhaupt tragen die wenigen Tiefen unter $50 \mathrm{~m}$ gern diesen Charakter, so zwei Löcher zwischen Sprogö und Korsör mit 58 m, eins zwischen Asnäs Riff und Horse Klint mit $\check{5} \mathrm{~m}$, eins bei Hatter Barn mit $\check{5} \mathrm{~m}$. Über die gesamte Terteilung der Tiefen geben die folgenden Zahlen Auskunft, die nach einer früheren Arbeit von mir zusammengestellt sind und um eine Neuberechnung des Sundes auf der gleichen Karte nach denselben Gesichtspunkten vermehrt wurden. ${ }^{1}$ )

Areale der Tiefenstufen in Quadratkilometern:

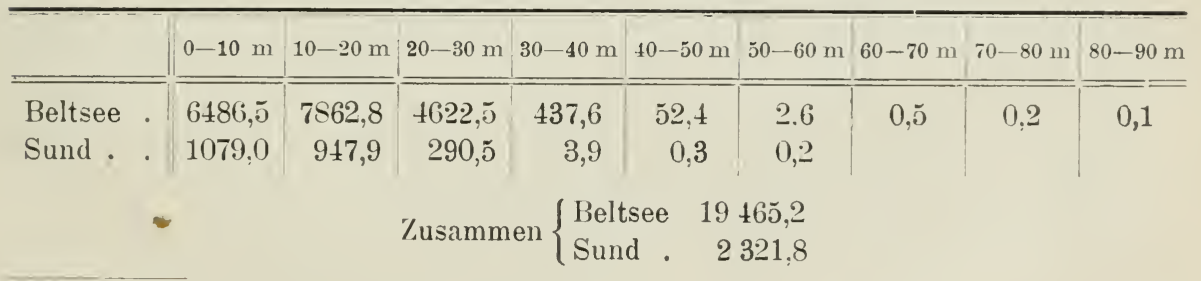
II , 1911.

1) H. Spethmann. Tiefenkarte der Beltsee. Peterm. Mitteilungen, 57. Jahrg., 
H. Spethmann.

Lănge der Isobathen in Kilometern:

\begin{tabular}{|c|c|c|c|c|c|c|c|c|c|}
\hline & $0 \mathrm{~m}$ & $10 \mathrm{~m}$ & $20 \mathrm{~m}$ & $30 \mathrm{~m}$ & $40 \mathrm{~m}$ & $50 \mathrm{~m}$ & (i) $\mathrm{m}$ & $70 \mathrm{~m}$ & $80 \mathrm{~m}$ \\
\hline Beltsee & 3507,9 & 3617,7 & 2790,9 & 748,4 & $176,7)$ & 19,6 & 20 & 2,0 & 1,0 \\
\hline sund. & 399,0 & $5: 3: 2,0$ & 24,0 & 633,0 & 10,5 & 1,0 & & & \\
\hline
\end{tabular}

Flächen der Isobathenareale in Quadratkilometern:

\begin{tabular}{|c|c|c|c|c|c|c|c|c|c|}
\hline & () $\mathrm{m}$ & $10 \mathrm{~m}$ & $20 \mathrm{~m}$ & $30 \mathrm{~m}$ & $40 \mathrm{~m}$ & $50 \mathrm{~m}$ & $60 \mathrm{~m}$ & $70 \mathrm{~m}$ & $80 \mathrm{~m}$ \\
\hline Beltsee & 19465,2 & $1: 978,7$ & ธ115,9 & 493,4 & 55,8 & 3,4 & 0,8 & 0,3 & 0.1 \\
\hline Sund. & 2321,8 & $1 \geq 42, S$ & 294,9 & 4,4 & 0,5 & 0.2 & & & \\
\hline
\end{tabular}

Bōschung in I'romille:

\begin{tabular}{|c|c|c|c|c|c|c|c|c|}
\hline & $0-10 \mathrm{~m}$ & $10-20 m$ & $20-30 \mathrm{~m}$ & $30-40 m$ & $40-50 \mathrm{~m}$ & $50-60 \mathrm{~m}$ & $60-70 m$ & $70-80 \mathrm{~m}$ \\
\hline Beltsee & $\check{5}, \check{c}$ & 4.1 & 3,8 & 10,6 & 18,7 & 41,5 & 40,0 & 75,0 \\
\hline Sund & 4.3 & 4,0 & 4,9 & 94,4 & 193,3 & $25, \tilde{5}$ & & \\
\hline
\end{tabular}

Bōschung in Bogenmaß:

\begin{tabular}{l||c|c|c|c|c|c|c|c|c|c|}
\hline & $0-10 \mathrm{~m}$ & $10-20 \mathrm{~m}$ & $20-30 \mathrm{~m}$ & $30-40 \mathrm{~m}$ & $40-50 \mathrm{~m}$ & $50-60 \mathrm{~m}$ & $60-70 \mathrm{~m}$ & $70-80 \mathrm{~m}$ \\
\hline $\begin{array}{l}\text { Beltsee } \\
\text { Sund }\end{array}$ & $0^{0} 19^{\prime}$ & $0^{0} 14^{\prime}$ & $0^{0} 13^{\prime}$ & $0^{0} 36^{\prime}$ & $1^{\prime} 04^{\prime}$ & $2^{0} 23^{\prime}$ & $2^{\circ} 18^{\prime}$ & $4^{\circ} 17^{\prime}$ \\
$0^{\prime \prime} 14^{\prime}$ & $0^{\prime} 17^{\prime}$ & $5^{0} 24^{\prime}$ & $10^{0} 57^{\prime}$ & $1^{0} 25^{\prime}$ & &
\end{tabular}

Der ozeanographischen Bedeutung halber fügen wir hinzu:

Volumen in Kubikkilometern:

\begin{tabular}{|c|c|c|c|c|c|c|c|c|}
\hline & $0-10 \mathrm{~m}$ & $10-20 \mathrm{~m}$ & $20-30 \mathrm{~m}$ & $30-40 m$ & $40-50 \mathrm{~m}$ & $50-60 \mathrm{~m}$ & $60-70 \mathrm{~m}$ & $70-80 \mathrm{~m}$ \\
\hline Beltsee & 162,220 & 90,473 & 28,047 & 2,746 & 0296 & 0,021 & 0,006 & 0,002 \\
\hline Sund . & 17,823 & 7,689 & 1,497 & $0,02 \bar{\jmath}$ & 0,004 & 0,001 & & \\
\hline \multirow[t]{2}{*}{ Sumine } & 180,043 & 98,162 & 29,544 & 2,771 & 0,300 & 0,022 & 0,006 & 0.002 \\
\hline & & \multicolumn{2}{|c|}{ Zusammen } & $\left\{\begin{array}{l}\text { Beltsee } \\
\text { Sund }\end{array}\right.$ & $\begin{array}{r}283,811 \\
27,039 \\
310850\end{array}$ & & & \\
\hline
\end{tabular}

Als mittlere Tiefen ergeben sich für die Beltsee $14,6 \mathrm{~m}$, für den Sund $11,6 \mathrm{~m}$, für beide zusammen bei einem Areal von $21787,0 \mathrm{~km}$ $14,3 \mathrm{~m}$.

Ehe wir die Darstellung des Reliefs verlassen, wollen wir noch einen Blick auf das nördlich und östlich anstoßende Gelände werfen.

Das Kattegat im Norden der Beltsee und des Sundes wird durch die Linie Läsö-Anholt in zwei verschiedene Tiefenregionen geteilt. Von Süden anfangend, ist es in seiner östlichen Hälfte bis zur Höhe von Anholt $25-35 \mathrm{~m}$ tief. Aber schon bei dem Großen Mittelgrund, der 
zwischen Anholt und Kullen liegt und sich bis auf $6,7 \mathrm{~m}$ erhebt, nimmt eine weiter im Süden noch schmale Rinne von unter $40 \mathrm{~m}$ Tiefe an Breite zu und geht bereits auf $60 \mathrm{~m}$ hinab. Nördlich von Anholt liegt ein etwa ebenso großer Teil dieser Hälfte des Kattegats unter wie über $40 \mathrm{~m}$, oft unter $60 \mathrm{~m}$. An der Westseite des Kleinen Mittelgrunds sind zwei Stellen mit $-92 \mathrm{~m}$ und $-100 \mathrm{~m}$ auf den deutschen Seekarten verzeichnet, an der Ostseite eine mit $-80 \mathrm{~m}$. Nördlich vom Kleinen Mittelgrund liegt bereits eine große Fläche zwischen $-40 \mathrm{~m}$ und $-60 \mathrm{~m}$ mit Löchern bis $\mathrm{zu}-116 \mathrm{~m}$. Westlich von ihr ist der Boden in seiner Gesamtheit flach, sich bis auf $-6,6 \mathrm{~m}$ hebend, aber dazwischen sind Rinnen und Löcher bis - 116, - $117 \mathrm{~m}$ und $-124 \mathrm{~m}$ eingeschaltet. Nördlich von Läsö breitet sich dann ein geschlossenes Tief von $-50 \mathrm{~m}$ aus, das gelegentlich unter $-100 \mathrm{~m}$ hinabgeht. Die Seitenbuchten. Laholm Bucht und Skelder Wiek sind untief und über $-20 \mathrm{~m}$. Bedeutend flacher ist die Westhälfte des Kattegats. Bis zur Höhe von Läsö tritt keine Tiefe unter $-40 \mathrm{~m}$ auf. Erst dort stellt sich eine Rinne mit größerem Ausmaß ein, die sich nach Osten wendet und nördlich von sich bis Skagen ein Terrain zwischen - 30 und - $40 \mathrm{~m}$ frei läßt, auf dem das Hertha Flach (-16 m) aufsitzt.

In der Arkonasee treten erst nördlich einer Linie Arkona-Grönsund wieder größere Tiefen mit $20 \mathrm{~m}$ ein, die mit Ausnahme der Möenbank, auf der noch einmal eine größere Fläche bis über - $20 \mathrm{~m}$ hinausgeht, nicht wieder überschritten werden. Südlich der Möenbank setzt die $40 \mathrm{~m}$ Isobathe wieder ein, die jetzt ein groBes geschlossenes Gebiet umspannt. Einige isolierte Flächen gehen in ihrem Bereich bei Trelleborg unter $-40 \mathrm{~m}$. Zunächst dehnt sich das große $-40 \mathrm{~m} \mathrm{Ge-}$ biet als Arkona-Becken zwischen Arkona, Möen Bank, Bornholm und Sandhammar aus, vereinzelt schon unter $-50 \mathrm{~m}$ steigend, wie nahe dem Kabel Arkona-Trelleborg ( $-58 \mathrm{~m})$. Während es sich nördlich von Bornholm allmählich weitersenkt, zieht sich südlich von Bornholm eine flache Schwelle nach Rügen hin, deren Mitte der Adlergrund aufsitzt ( $-6 \mathrm{~m})$. Ist zwischen Adlergrund und Bornholm der Schwellencharakter scharf entwickelt, so geht er südlich davon verloren, da von der Odermündung fast bis zur Höhe von Jasmund und bis nach Kolberg reichend eine große Dreiecksfläche $10-20 \mathrm{~m}$ tief ist, in der sich die Oderbank mit $-6 \mathrm{~m}$ ausdehnt. In der Dreiecksfläche fällt die tiefe Rinne außerhalb der Prorer Wiek auf, die in der Fortsetzung der Peene- oder Odermündung gelegen ist. 


\section{Die Gewinnung der täglichen Beobachtungen.}

I) Le Lage der Feuerschiffe Schultz Grund, Gjedser Rev, I)rogden und Lappe Grund S. 10. - Jie Vornahme der Beobachtungen S. 11. - I)ie Genauigkeit der Beobachtungen S. 12 .

In unserm Gebiet liegen vier Feuerschiffe, die zugleich auch ozeanographische Stationen sind, Schultz Grund, Gjedser Rev, Drogden, Lappo Grund. Schultz Grund-Feuerschiff befindet sich am Nordausgang des Samsö Beltes zwischen Seelands Riff und Schultz Grund. Zwischen beiden zieht sich eine $30 \mathrm{~m}$ bis teilweise unter $40 \mathrm{~m}$ tiefe Rinne hindurch, an deren Nordwestseite das Fahrzeug bei 27,3 m Tiefe derart verankert ist, daß es am Abhang der Rinne schwimmt. Von ihm bis zur Mitte der Rinne wird keinen ganzen Kilometer betragen. Das Schiff liegt frei mit Ausnahme des Winkels zwischen Westnordwest und Nordnordost, wo sich der über $-10 \mathrm{~m}$ ansteigende Schultz Grund ausdehnt, der an seiner flachsten Stelle, die gerade nordwärts gelegen ist, nur $3,5 \mathrm{~m}$ Wassertiefe hält. Das nächste Land ist im Südsüdwesten in etwa $16 \mathrm{~km}$ Entfernung, es ist die Spitze von Seeland Odde. Da diese Landzunge niedrig ist, ist das Leuchtschiff allen Winden ausgesetzt; nur jene aus Süden und Westen stehen etwas, wenn auch nur ganz gering, in Lee. Strömungen vermögen ebenfalls von allen Seiten heranzukommen, aber nicht in gleichem Maße. Am wenigsten gehindert vermag sich das Wasser von etwa Südwesten nach Nordosten zu bewegen, sonst ist verschiedener Flachs halber keine durchlaufende Richtung mehr vertreten; jene nach Südsülosten und in geringerem Maße jene nach Westen gewähren noch eine freie Passage.

Das Feuerschiff Gjedser Rev ist nicht bei Gjedser zu finden (dort ist das Feuerschiff Gjedser Havn gelegen), sondern halbwegs zwischen Gjedser Odde, der Südostspitze Falsters, und dem Fischland am östlichen Grenzgebiet der mecklenburgischen Küste. Es ist ziemlich gleich weit von Dänemark und von Deutschland entfernt und schwimmt vor dem äußersten Vorposten eines Riffes, das submarin als Fortsetzung des Höhenzuges an der Westseite des südlichen Falster sich in NordwestSüdostrichtung in die 0stsee erstreckt. Nirgends geht es bei ungefähr $17 \mathrm{~km}$ Länge unter $10 \mathrm{~m}$ Tiefe hinab, erst dann hört es schnell auf, so daß sein äußerstes Ende fast an die Kadetrinne stößt. Diese zieht, schon näher der deutschen Seite gelegen, in leichtem Bogen von Nordnordost nach Südwesten mit Tiefen von fast $30 \mathrm{~m}$ südöstlich um das Feuerschiff herum. Letzteres ist durch seine exponierte Lage allen Winden zugänglich, wobei jene aus Nordosten und Südwesten die größte 
Wasserfläche bestreichen können, ehe sie zum Fahrzeug gelangen. Auch das Wasser hat allseits ungehinderten Zutritt, doch werden bei ihm infolge der hohen Lage des Riffes Strömungen, die aus Westen kommen, etwas nach Südosten, und solche, die aus Nordosten kommen, etwas nach Süden abgelenkt.

Das Feuerschiff Drogden liegt am Südeingang zur Drogdenrinne dicht südlich des $6 \mathrm{~m}$ flachen Quartus Grundes. Es befindet sich gleichfalls frei nach allen Seiten, nur nach Nordwesten und Nordosten bieten Amager und Saltholm ganz geringen Landschutz. Der Strom vermag sich gemäß der rinnenartigen Gestaltung des Reliefs in der Hauptsache nur aus nördlichen oder südlichen Richtungen zu bewegen. Feuerschiff Lappe Grund besitzt am meisten Landschutz, da es nur wenig nördlich der Sundenge von Helsingör-Helsingborg ausgelegt ist. Ziemlich in Strommitte gelegen, vermag das Wasser in der Hauptsache bei ihm gleichfalls nur aus zwei Richtungen zu passieren, aus Südsüdosten und Nordnordwesten.

Auf sämtlichen vier Feuerschiffen, ferner noch im Kattegat auf Anholt Knob, Läsö Rende und Läsö Trindel, Skagens Rev sowie auf Kobbergrund resp. Östre Flak erfolgen die Beobachtungen seit dem 1. November 1902 nach einheitlichen Gesichtspunkten, die sich gänzlich denen der internationalen Meeresforschung anschließen, so daß die Resultate gegenseitig verglichen werden können. Mit diesem Zeitpunkt beginnt eine ununterbrochene Reihe gleichartig durchgeführter Beobachtungen, deren Aufzeichnungen beim AbschluB dieser Arbeit in dem jährlich erscheinenden Nautisk-Meteorologisk Aarbok, das das dänische meteorologische Institut zu Kopenhagen herausgibt, bis zum 31. Dezember 1911 veröffentlicht vorliegen.

Auf den Feuerschiffen, von denen für unser Gebiet die vier näher beschriebenen in Frage kommen, werden mit Beginn jeder Wache die Lufttemperatur (C) und der Wind nach Richtung (16 KompaBstrichrose) und Stärke (Beaufort) gemessen; ebenso wird vierstündlich die Bedeckung des Himmels geschätzt. Von hydrographischen Arbeiten geschieht vierstündlich die Feststellung des Oberflächenstromes nach Stärke (Seemeile in Stunde) und Richtung (16 Kompaßstrichrose). Außerdem wird morgens $8 \mathrm{Uhr}$ eine Temperatur und Salzgehaltsserie aus verschiedenen Tiefen in je $5 \mathrm{~m}$ Abstand und rom Boden genommen, so bei Schultz Grund aus $0 \mathrm{~m}, 5 \mathrm{~m}, 10 \mathrm{~m}, 15 \mathrm{~m}, 20 \mathrm{~m}, 26 \mathrm{~m}$, bei Lappe Grund aus $0 \mathrm{~m}, 5 \mathrm{~m}$, $10 \mathrm{~m}, 15 \mathrm{~m}, 20 \mathrm{~m}, 23 \mathrm{~m}$, bei Gjedser Rer aus $0 \mathrm{~m}, 5 \mathrm{~m}, 11 \mathrm{~m}$ und bei Drogden von 1902 bis 1908 aus $0 \mathrm{~m}, 3 \mathrm{~m}, 6 \mathrm{~m}$, später aus $0 \mathrm{~m}$, 5 $\mathrm{m}, 8 \mathrm{~m}$. 
An Instrumenten werden benutzt: Lompaß und teilweise Log zur Schätzung und Messung von Stromrichtung und Stromstärke; halbgradteilige Thermometer für die Temperatur der Luft und des Oberflächenwassers, guttaperchierte Thermometer nach Negretti und Zambra für das Tiefenwasser. Sämtliche Thermometer sind aus Jenaer Normalglas 16 III hergestellt und monatlicher Kontrolle seitens des dänischen meteorologischen Instituts unterworfen. Zum Wasserschöpfen dienen Wasserflaschen ron Arwidson \& Rung. Das spez. Gewicht wird mit kontrollierten Glasaräometern bestimmt, die in Glaszylindern abgelesen werden, welche in Balanzierbügeln schwingen. Die Reduktion der Aufzeichnungen auf $17,5^{\circ}$ und die Umwertung des Salzgehaltes in Zehntel Promille nach Knudsens Tabellen geschieht in Kopenhagen. Zur Kontrolle wird mit Ausnahme auf Drogden zweimal in der Woche die gemessene Wasserprobe des Bodens der Station in Flaschen eingefüllt, um gleichfalls in Kopenhagen titriert zu werden. ${ }^{1}$ ) Diese vergleichenden Kontrollbestimmungen hat I. P. Jacobsen zur Mittelbildung der Differenzen zwischen Titrierungen und Aräometerbeobachtungen benutzt. ${ }^{2}$ ) Es ergeben sich positive und negative Abweichungen, die im Monatsmittel niemals $1^{0}{ }_{00}$ erreichten. Als Gesamtmittel für die Beobachtungen auf allen Feuerschiffen wurde $+0,2^{\circ} \%_{00}$ ermittelt. Wir stellen im folgenden aus Jacobsens Berechnungen eine Tabelle für die Abweichungen der einzelnen Jahresmittel auf den Stationen, die für uns in Betracht kommen. zusammen:

\begin{tabular}{c|c|c|c}
\hline Station & Schultz Grund & Lappegrund & Gịedser Rev \\
\hline Tiefe & $26 \mathrm{~m}$ & $23 \mathrm{~m}$ & $11 \mathrm{~m}$ \\
\hline \hline 1902 & 0,20 & 0,10 & 0,53 \\
1903 & 0,25 & 0,07 & 0,70 \\
1904 & 0,21 & 0,04 & 0,67 \\
1905 & $-0,05$ & 0,02 & 0,62 \\
1906 & $-0,07$ & 0,13 & 0,47
\end{tabular}

Die Abweichungen sind bei Gijedser Rev am stärksten; sieht man die Einzelbeobachtungen dieser Station ein, so begegnet man unter ihnen sehr selten Abweichungen über $1 \%$, bei den übrigen Stationen selten über $0,5^{0}{ }_{00}$. Im allgemeinen ist zu erkennen, daß die Differenzen um

1) Zusammengestellt nach den einleitenden Angaben im Nautisk-Meteorologisk Aarboli, siehe bes. Jahrg. 1902.

$\left.{ }^{2}\right)$ I. P. Jacobsen, Mittelwerte von Temperatur und Salzgehalt, bearbeitet nach hydrographischen Beobachtungen in Dänischen Gewässern 1880-1907. Medd. Kommission f. Havunders. Kopenlıagen 1908. 
eine Konstante nur wenig schwanken, doch genügt das vorliegende Beobachtungsmaterial noch nicht, diese als Reduktion zu verwerten, da es sich nur auf eine kurze Zeit beschränkt und da ferner bei den vorstehenden Stationen es sich lediglich auf je eine Tiefe bezieht, die Auswertungen für die Feuerschiffe Horns Rev, Skagens Rev und Läsö Rende, für welche Beobachtungen aus verschiedenen Tiefen vorliegen, aber bekunden, daß die Abweichungen, wenn auch in geringem Ausmaß, in den einzelnen Tiefen ein und derselben Station schwanken. Als Resultat ergibt sich, daß die jeweiligen Feuerschiffsbeobachtungen im Vergleich mit anderen Beobachtungen bis zu $0,5^{0}{ }_{00}$ für die einzelne Beobachtung mit Ausnahme für Gjedser Rev, wo hierfür $1 \%$ zu setzen ist, als ungenau angesehen werden können. Für Monatsmittel und Jahresmittel verringert sich dieser Wert naturgemäß. Als Ganzes genommen, ist den umsichtig organisierten und kritisch durchgeführten Beobachtungen, deren dichte Reihenfolge ein im Norden Europas fast einzig dastehendes ozeanographisches Material geschaffen hat, jedoch rolles Vertrauen entgegenzubringen.

\section{Sturm und Strom.}

Wind und Strom S. 13. - Die angewandte Methode S. 14. - Drogden S. 16. - Schultz Grund S. 18. - Lappe Grund S. 18. - Gjedser Rev S. 19. - Gesamtbild für die vier Feuerschiffe S. 20. - Die Stromstärke S. 22. - Der hydrographische Wert S. 23. Tabellen S. 24.

Für die Salzgehaltsverteilung und die Strömung ist sicherlich der Wind ein maßgebender Faktor. Eine Berechnung des Anteils von 16 Windrichtungen am einlaufenden und auslaufenden Strom muB zu gewissen überwiegenden Prozentzahlen für einzelne Richtungen führen. Ich habe zunächst versuchsweise diese Arbeit für Lappegrund und den größeren Teil für Gjedser Rev für die Periode 1902 bis 1911 auf Grund der 6stündigen Beobachtungen von Wind und Stromrichtungen durchgeführt, insgesamt für mehr denn 30 Tausend Beobachtungspaare, fand dann aber bei den großen Schwankungen von Jahr zu Jahr und Monat zu Monat, daß das Gesamtergebnis so sehr durch Einzelheiten belastet wird, daß aus ihm keine strengen Schlubfolgerungen gezogen werden können, sondern nur mit gewissen Wahrscheinlichkeiten für das Eintreffen von Auslauf oder Einlauf bei dieser oder jener Windrichtung gerechnet werden kann. Hierzu kam ein anderes Bedenken, das mich schließlich von dieser Arbeit abgehen ließ. Der weitaus größte Teil der Windstärken gehört mittelstarken und schwachen Luftbewegungen oder 
Windstillen an. Ich kamn ihren hydrographischen Effekt wohl für die l'ositionen der einzelnen Fenerschiffe herechnen, erziele aber damit kein Ergebnis für die Bewegungen in der Beltsee und im Sund. Stelle ich Einlauf bei einer Windrichtung an einer Station fest, so kann sich bei ihr Auslauf auf der gegenüberliegenden Seite der Wasserstraße vollziehen, ohne daß es irgendwie in den Beobachtungen zum Ausdruck käme; und sie mit anderweitigen gegenwärtig in Beziehung zu setzen, fehlt vorläufig jegliches Material. Oder der beobachtete Einlauf mag in Wirklichkeit überhaupt gar kein Einlauf sein, sondern das Feuerschiff liegt im Gebiet eines resultierenden Stromes, wie beispielsweise eines Neerstromes, so daß der beobachtete Einlauf in Wirklichkeit Auslauf ist. Die mittleren und kleinen Windstärken sind eben so wenig einheitlich durchgreifende Faktoren für eine größere Wasserfläche, Strömungen, die sie an einer Stelle entwickeln oder die unabhängig von ihnen vor sich gehen, stehen in einem in der Verteilung von Land und Wasser so buntem Gebiet wie das hier behandelte unter dem Einfluß so mannigfaltiger Wirkungen, daß wir aus vier für die Ozeanographie ziemlich zufällig gelegenen Stationen, deren hydrographische Umgebung so gut wie unbekannt ist, gar keine Schlußfolgerung für die Gesantheit der Beltsee und den Sund zu ziehen berechtigt sind.

Anders liegen die Verhältnisse, wenn wir die kräftigen Luftzirkulationen ins Auge fassen. Stürme sind hydrographisch durchgreifende Faktoren. Wenn wir sie mit Strombewegungen in Verbindung setzen, so ist ein scharf umrissenes Bild zu erwarten, das uns den Schlüssel für Bewegungen in der ganzen Beltsee gibt.

Zu dem Zweck habe ich die Stürme nach ihrer Beeinflussung der Stromrichtung, ob Auslauf oder Einlauf erzeugend, gruppiert. Als Stürme sind sämtliche Windstärken von 7 Beaufort an aufwärts zusammengefaßt. Ich habe zuerst nur die Luftbewegungen von Stärke 8 an aufwärts benutzen wollen, sah aber bald nach orientierenden Studien, daß Stärke 7 schon von gleich großem hydrographischem Effekt ist. Die Dauer der Stürme wurde gemäß den vierstündigen Beobachtungen in Wachen angegeben. ihre Herkunftsrichtung rechtsdrehend, so daB W-E bedeutet, daß der Sturm aus der Richtung zwischen W-E über N geweht hat, ohne daß damit ausgedrückt wird, ob er links- oder rechtsdrehend war. Die Angabe soll sich also lediglich auf das bestrichene Areal der Windrose beziehen. Schwierigkeiten bot mitunter, die einzelnen Stürme so zu begrenzen, daß ihr Einfluß auf das Wasser doch in einer Mittelbildung klar zum Ausdruck kommt. Nach mancherlei Versuchen habe ich mich zu folgendem Verfahren entschlossen. 
Trat zwischen zwei oder mehr Wachen, bei denen Windstärke 7 oder mehr wehte, auf einer einzigen Wache eine geringere Windstärke ein, so wurde der meistens nur vorübergehend etwas abflauende Sturm als ununterbrochen angesehen und als einziger gezählt. Bei der Feststellung der Zahl der Wachen, an denen er wirkte, wurde hingegen die Wache unter 7 Beaufort nicht mit einbegriffen. In einigen Fällen wurde derart bei größeren Stürmen ein Abflauen auch während zweier Wachen behandelt; es waren längere Sturmperioden, bei denen der Wind während zweier Wachen entweder nur bis Sturmstärko 6 herabging oder während des Passierens des Minimums aussetzte. Es ist bei Drogden achtmal, bei Schultz Grund sechsmal, bei Lappe Grund siebenmal und bei Gjedser Rev elfmal geschehen, so daß insgesamt $105 \check{3}$ Stürme gezählt wurden. In der ersten Kolumne in den Tabellen S. 24-31 sind die Zahl der Stürme nach Jahreszeiten geordnet angegeben, die Extreme ihrer Dauer nach Wachen.

Die beiden Stromrichtungen sind den Wachen entnommen, an denen jeweils der Sturm herrschte, d. h. Windstärke 7 und mehr zu verzeichnen war. Zu dem Zwecke wurden die Stromrichtungen nach "Auslauf und Einlauf" zusammengefaßt und berechnet. Die Entscheidung, ob Auslauf oder Einlauf vorlag, war mit Ausnahme bei Schultz Grund leicht zu treffen; bei diesem wurde NNW-SE als Einlauf, SSE-NIV als Auslauf aufgefaßt. Entsprechend der herrschenden Stromrichtung wurden nunmehr die Sturmrichtungen in Kolumnen für Einlauf, Auslauf und Stromstille oder für die verschiedenen Übergangsstadien dieser Phasen eingeordnet. Gleichzeitig vorgenommene Berechnungen, auch die gesamte Einwirkung des Sturmes auf den Strom festzulegen, d. h. auch für die Wirkung nach Abflauen unter Stärke 7, führten zu keinem greifbaren Ergebnis, da oft schwache Winde, die nach dem Sturm auftraten, den Einlauf oder Auslauf zeitlich verlängerten, ohne daß es möglich gewesen wäre, nur einigermaßen deutlich die Grenze des Sturmcinflusses aufzufinden. Desgleichen haben Zusammenstellungen über das Maximum der Stromstärke kein klares Bild über das Eintreten derselben zu geben vermocht. Indem die Stürme oft zwischen Stärke 7 bis 10 schwanken, schwillt auch die Stromstärke an oder ab, ohne dalj sich in der Regel ein deutliches Maximum entwickelt. Ebenso hat die gleichzeitige Berücksichtigung über das $\mathrm{Zu}$ - und Abnehmen des Salzgehaltes keine genügende Aufklärung über den hydrographischen Sturmeinfluß gebracht. Wohl ließ sich in vielen Fällen ein Maximum oder Minimum am nächsten Morgen feststellen, also augenscheinlich mit einer Verzögerung, doch ist hierbei zu bedenken, daß die Windbeobach- 
tungen vierstündig, die Salzgehaltsbeobachtungen aber nur vierundzwanzigstündig angestellt werden, so dab begreiflicherweiso schon aus diesem Grunde die Extreme des Salzgehaltes immer etwas später gefunden werden mußten.

Betrachten wir nunmehr den EinfluB der Sturmrichtung, so wollen wir zunächst die Feuerschiffe einzeln besprechen und mit Drogden beginnen, bei dem sich ungezwungen zwei große Gruppen ergreben. SSW-NNE-Sturm beschert Einlauf, Sturm aus NNE-SW Auslauf. Diese Aufteilung läßt sich noch schärfer fassen. NNE bringt nur ein einziges Mal Einlauf, am 5. Februar 1907. Der Einlauf ist schwach, 0.5 Knoten in der Stunde, und geht bei Abflauen des gleichen Windes in Auslauf über, der sonst immer bei NNE herrscht. In die Übergangsgebiete zwischen Einlauf und Auslauf schalten sich bei Stürmen die Kalmen des Stromes ein. Die meisten von ihnen sind zwischen s und SW gelegen, nur am 22. Februar 1908 tritt Stromstille auch bei SSE ein, es wehte kurz vorher und nachher aus S-SW. Eine andere kleinere Gruppe von Kalmen ist um N gelegen, je einmal kei N und NNIT. Für diese liegen zwei schöne Beispiele vom 25.-26. November 1904 and vom $14 .-17$. November 1905 vor. Das erste sei hier wiedergegeben. Es bedeutet A Auslauf, E Eir`auf, C Stromstille. Die Geschwindigkeit ist als Knoten in der Stunde verstanden.

\begin{tabular}{|c|c|c|c|c|c|c|c|}
\hline & Nor. 04 & $4 \mathrm{a}$ & $8 a$ & Mittag & $4 \mathrm{p}$ & $8 p$ & Mitternacht \\
\hline \multirow[t]{2}{*}{ Wind } & 25. & NNE 3 & NNE 4 & NNE 5 & N 8 & $N: 9$ & N 8 \\
\hline & 26. & $\times 7$ & N 6 & N 4 & N 4 & $\mathrm{~N} i$ & NE 6 \\
\hline \multirow[t]{2}{*}{ Strom } & 25. & A 0,8 & A 1,5 & A 1,8 & A 0.5 & A 0,2 & \\
\hline & 26. & C & C & C & C & $\mathrm{C}$ & A 0,5 \\
\hline
\end{tabular}

Für die südliche Richtung stellen die gleichen Tage vom Jahre 1907 einen guten Beleg dar:

\begin{tabular}{|c|c|c|c|c|c|c|c|}
\hline & Nor. 07 & $4 a$ & $8 a$ & Mittag & $4 p$ & $8 p$ & Mitternacht \\
\hline \multirow[t]{2}{*}{ Wind } & 25. & SSW 5 & SSIT 6 & SSIV 5 & SSE 6 & SSE 8 & S 8 \\
\hline & 26. & S S & S 5 & SSIV 7 & WSW 6 & IV 3 & S 2 \\
\hline \multirow[t]{2}{*}{ Strom } & 25. & C & C & C & E 0,3 & E 0,2 & C \\
\hline & 26. & $\mathrm{C}$ & $\mathrm{C}$ & $\mathrm{C}$ & E $0, \overline{5}$ & E 1,3 & E 1,0 \\
\hline
\end{tabular}

Auch bei starkem SIV tritt ein ähnliches Strombild ein, wie der 25.-26. Januar 1906 oder der 8.-9. Februar 1906 zeigten. Außerdem ist einmal bei Sturm aus W Stromstille wahrgenommen, am 24. August 1903. Zur Beobachtungszeit des nur eine Wache dauernden Sturmes, mittags 12 Uhr, kenterte gerade der Strom von Auslauf in Einlauf, so 
daß demnach auch dieser Weststurm sich der generellen Anordnung vom Auslauf einfügt.

Tritt bei Drogden im allgemeinen bei Stürmen von SSW über W nach N Einlauf, von NNE über E nach SW Auslauf, im Grenzgebiet Stromstille ein, so werden uns ohne weiteres die Strombewegungen bei Stürmen verständlich, die sich von dem einen dieser Gebiete der Windrose in das andere bewegen. Bei Rechtsdrehung muß immer einem Auslauf ein Einlauf folgen, wenn es sich um den Südrand der Windrose handelt. Unter den acht in der Tabelle angeführten Fällen kommen sieben auf diese Gruppe, wobei allerdings zweimal der Auslauf bei WSW herrscht, einmal am 3. Mai 1904 und einmal am 31. August 1903. An beiden Tagen wehte die Wache vorher normal SW und war die Abweichung nur auf je einer Wache angetroffen. Für die normale Abwickelung des Wechsels der Stromrichtung in der Abhängigkeit von den Stürmen geben die Tage rom 12.-13. März 1906 ein klares Detailbild.

\begin{tabular}{|c|c|c|c|c|c|c|c|}
\hline & März 06 & $4 \mathrm{a}$ & $8 a$ & Mittag & $4 p$ & $8 p$ & Mitternacht \\
\hline \multirow[t]{2}{*}{ Wind } & 12. & & & SSIV 10 & WSIV 10 & WSW 10 & WSW 10 \\
\hline & 13. & WNIV 9 & WNW 8 & W 9 & WNW 9 & W 7 & SW 5 \\
\hline \multirow[t]{2}{*}{ Strom } & 12. & A 1,3 & A 1,0 & A 0,3 & C & E 1,3 & E 2,3 \\
\hline & 13. & E 2,5 & $\mathrm{E} 2,5$ & $\mathrm{E} 2,5$ & $\mathrm{E} 2, \overline{5}$ & $\mathrm{E} 1,7$ & E 0,5 \\
\hline
\end{tabular}

Linksdrehung muß dagegen am Nordrand der Windrose vor sich gehen, wenn der gleiche Effekt erzielt werden soll, was bei dem übrigbleibenden achten Fall aus dem Frühjahr 1903 zutrifft. Der Vorgang, daß einem Einlaiuf ein Auslauf folgt, müßte sich in umgekehrtem Sinne vollziehen, er ist während der Beobachtungsperiode aber nicht eingetreten. Dagegen hat sich der Ubergang vom Auslauf resp. Einlauf in Stromstille mehrfach abgespielt, und zwar, wie die Tabelle sofort zeigt, in einer Weise, wie es bei Drogden beim Drehen eines Sturmes in das Grenzgebiet der Sturmrichtung zu erwarten ist. Nur für den 7.- 8. Januar 1908 trifft diese Erklärung nicht zu. Es blies ein Sturm aus SW zunächst drei Wachen, bei dieser Richtung trat bei der zweiten Wache Stromstille ein, während vorher und nachher Einlauf herrschte. Ebenso sind die Fälle ohne weiteres verständlich, an denen Stromstille in Einlauf übergeht. Sie liegen wiederum im Grenzgebiet der Sturmrichtung. Hingegen scheinen die beiden Fälle des Überganges von Stromstille in Auslauf auf den ersten Blick aus dem allgemeinen Bild herauszufallen, indem auf einen rechtsdrehenden WSTV resp. SSW ein Auslauf erfolgte. Sehen wir unś die beiden Stürme nach ihren einzelnen Beobachtungen an, so finden wir bei beiden" nur ein etwas verzögertes 
Eintreten der zu erwartenden Richtung, bei dem einen vom 5.-6. Oktober 1903 nur einen kurzen Auslauf bei Weststurm, der sich sofort in Einlauf verwandelt, bei dem andern vom 15.-16. Oktober 1909 ebenfalls ein sofortiges Übergehen in Einlauf.

Xhnlich einfach wie bei Drogden liegen dio Verhältnisse bei Schultz Grund. Hier bewegt sich die Sturmrichtung bei Einlauf zwischen S und ENE; nur rom 11.-12. November 1910 trat bei einem Sturm, der in drei Wachen von ESE auf ENE drehte, auch Einlauf in Erscheinung. Der Auslauf geschieht bei Stürmen zwischen $\mathrm{N}$ und $\mathrm{SW}$; nur bei jo einer Wache am 20. April 1903 und 21. April 1908 war auch Auslauf bei Sturm aus NNW, der jedoch gleich darauf in Einlauf überging. Der Übergang von Auslauf in Einlauf ordnet sich ganz dieser Verteilung ein, während bei dem Übergang von Einlauf zum Auslauf einmal bei SE Einlauf eintritt. Es ist einer der wenigen Fälle, wo während eines Sturmes Einlauf zwar in Auslauf übergeht, aber danach doch wieder die Oberhand gewinnt.

\begin{tabular}{l|c|c|c|c|c|c|c}
\hline & & $4 \mathrm{a}$ & $8 \mathrm{a}$ & Mittag & $4 \mathrm{p}$ & $8 \mathrm{p}$ & Mitternacht \\
\hline Wind & 12. & & & & & & SE 7 \\
Strom & $\begin{array}{l}13 . \\
12 .\end{array}$ & ESE 9 & E 9 & ENE 8 & N 8 & NNW 7 & \\
& 13. & A & A & A & A & E & E
\end{tabular}

Auch der Auslauf greift bei den Übergangsphasen von Einlauf zum Auslauf etwas weiter, zweimal bis NNW und einmal bis SSW. Stromstillen sind bei Schultz Grund als selbständige Erscheinung bei Stürmen überhaupt nicht zu finden, nur beim Kentern der Stromrichtung treten sie vorübergehend kurz auf, jedoch hierbei nur einmal in die Beobachtungszeit fallend, so daß Stromstille bei Sturm sich außerordentlich selten bei Schultz Grund zu ereignen scheint.

Bei Lappe Grund schließen die Gebiete der Sturmrichtungen bei Einlauf und Auslauf nicht mehr so scharf einander aus, wie an den beiden behandelten Feuerschiffen. Einlauf ist nur bei Stürmen zwischen SE und S nicht beobachtet, Auslauf ist sogar bei allen Sturmrichtungen erfolgt. Im allgemeinen tritt bei ihm Einlauf nur bei Stürmen zwischen SSW und NE auf, denn Südsturm wehte bei ihm nur während einer Wache am 6. April 1904, N-Sturm einmal während dreier Wachen am 3.April 1911 und NNE-Sturm während zweier Wachen am 12. April 1911. Auffallenderweise brachte ein Sturm aus ENE-ESE während der Dauer von 6 Wachen am 9.-10. Oktober 1903 und ein Sturm aus SE während 
6 Wachen vom 12.-13. Oktober 1903 Einlauf, während Stürme aus diesen Richtungen sonst immer Auslauf im Gefolge hatten.

Dieser trat hauptsächlich bei Sturmrichtungen aus NE-NNW auf, Auslauf bei Nordsturm entwickelte sich nur an einer Wache am 21. Juli 1905. Auffallend wenig waren Stromstillen $\mathrm{zu}$ notieren, nur zweimal bei $\mathrm{S}$ und einmal bei $\mathrm{SW}$, so daß sich aus diesen drei Fällen kein klares Bild ableiten läßt, ebenso, wie für die wenigen Übergänge von Stromstille in Auslauf resp. Einlauf. Gar nicht ereignet hat sich der Übergang von Auslauf in Einlauf, der umgekehrte Verlauf dagegen häufiger. Mit Ausnahme eines Nordsturmes am 30. August 1905, wo auf der Wache vorher NE wehte, setzt er immer in den Sturmrichtungen ein, die wir für Auslauf die Regel fanden, ebenso endet er immer in den Richtungen, die für Einlauf vorwalten.

Noch mehr als bei Lappe Grund greifen die Sturmrichtungen für Einlauf und Auslauf bei Gjedser Rev ineinander über. Bei ihnen tritt sowohl Einlauf wie Auslauf bei allen Sturmrichtungen auf, jedoch überwiegen gewisse Orientierungen, namentlich beim Einlauf, mit dem wir beginnen wollen. In der neunjährigen Periode vollzieht er sich im Frühjahr, Sommer und Herbst nur bei Stürmen von SW bis ENE. Bei ENE-Sturm hat er sich nur zweimal ereignet, am 25. März 1911 und am 17. April 1905, sonst ist seine Grenze schon bei NNE gelegen. Im Winter ist Einlauf bei Stürmen aus ESE bis NNW aufgetreten. Der ESE-Sturm wehte nur einmal während einer Wache am 8. Januar 1908, sonst ist Einlauf immer erst bei SSW-Stürmen erschienen, so daB, wenn wir die vorstehend aufgezählten drei Fälle aus dem Auge lassen, wir zu dem Ergebnis gelangen, daß Einlauf bei Stürmen zwischen SSW und NNE auftritt.

Der Auslauf beschränkt sich nicht auf eine halbe Windrose. Im Herbst tritt er bei allen Sturmrichtungen ein. Sieht man aber daron ab, daß nur ein einziges Mal am 9. Oktober 1905 während einer Wache bei NNW Auslauf erfolgte und ebenso am 13. des gleichen Monats, so bewegt sich im Herbst die Sturmrichtung, bei der Auslauf stattfindet, zwischen NE und WSW. Im Sommer liegt sie zwischen ENE und NW und im Frühling und Winter zwischen NNE und NW, wenn man je einen Nordsturm mit einer Wache Dauer am 26. Januar 1905 und am 3. April 1911 ausschaltet. Da sich also ergibt, daß bei Gjedser Rev Auslauf bei NNEbis NW-Stürmen erfolgt, Einlauf bei SSW- bis NNE-Stürmen, so gelangt bei Stürmen zwischen SSW und NW Einlauf sowohl wie Auslauf zur Ausbildung. In diese Zone fallen die Stromstillen, die bei Stürmen erfolgen; sie dehnen sich aber nach beiden Seiten etwas aus, so daß sich 
ihre größte Zahl \%wischen S und NNW legt. Am 22. Hezember 1909 war Stromstille anch bei einem Sturm aus SE bis SSE während zweier Wachen beobachtet. Mehrfach hat sich dagegen Stromstille auch bei Stürmen aus NE bis ENE eingestellt, so daß man für Giedser Rev Sagen muB, daß sich Stromstille bei Stürmen zwischen S bis NNW wie aus NE bis ENE ereignet.

Dementsprechend setzen aus diesen Himmelsrichtungen auch alle Stürme ein, die nach Stromstille Einlauf bringen, oder umgekehrt enden in diesen Richtungen, wenn einem Einlauf eine Stromstille folgt. Eine einzige Ausnahme ist vom 18. Juli $1907 \mathrm{zu}$ verzeichnen, wo ein WNW nach $\mathrm{N}$ übergehend nach Einlauf Stromstille im Gefolge hat, die aber nur der kurze C̈bergang zum Auslauf ist, wie die Stromrichtungen aus den nächsten Wachen bezeugen. Weniger klar liegen die Beziehungen zwischen Auslauf und Stromstille. Folgt diese dem Auslauf, so hat der Strom stets in die für sie normale Richtung gedreht mit einer einzigen Ausnahme aus dem Frühjahr 1904, wo er von ESE nach E übergehend die Grenze etwas überschreitet, umgekehrt aber, folgt der Auslauf der Stromstille, so treten unter den elf vorkommenden Fällen fünf Abweichungen ein, doch beruhen diese darauf, daß es sich meistens um Stürme handelt, bei denen mehrfach Stromstillen am Anfang und in der Mitte rorübergehend eingeschaltet sind. Waren sie nur während der Dauer eines Sturmes vorhanden, so sind sie der Zeit des Kenterns zwischen Einlauf und Auslauf oder umgekehrt zugefallen. Diese Stürme bewegen sich also alle in den normalen Bahnen, die wir aus der Mittelbildung gewanmen.

Faßt man den gesamten Eindruck zusammen, so ergibt sich zunächst, daß sich mit Ausnahme bei Gjedser Rev, wo die Verhältnisse etwas weniger übersichtlich waren, eine leichte Gruppierung der Sturmrichtungen nach Einlauf und Auslauf vornehmen läßt. Erwägt man, daß insgesamt 1053 Stürme zu ordnen waren, so dürften die wenigen Ausnahmen, die wir jeweils aufgezählt haben, kaum das allgemeine Bild stören. Dieses ist folgendes:

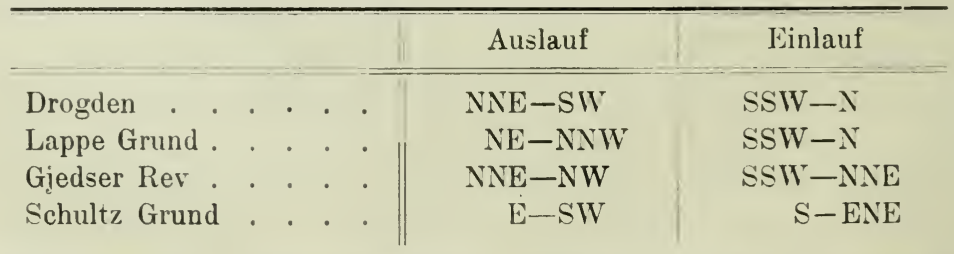

Einlauf erfolgt im allgemeinen zwischen SSW- und N-Stürmen. So verständlich er bei Stürmen aus $\mathrm{N}$ und NW ist, so sehr möchte 
uns zunächst überraschen, daß nicht nur bei Stürmen aus W, sondern selbst aus SSW entgegen der Windrichtung Einlauf in die Arkonasee orfolgt. Bei Schultz Grund erfolgt der Einlauf sogar bei Südsturm, jedoch nur viermal in den neun Jahren, so daß wir dieses Vorkommen nicht als generell bezeichnen können. Die andere Seite dieses Sturmgebietes schneidet zwischen $\mathrm{N}$ und ENE ab, und zwar ist gemäß der Lage der Wasserstraßen nur noch bei Nordsturm Einlauf bei Drogden und Lappe Grund, da bei ihnen unmittelbar im Osten größere Landflächen liegen, bei NNE-Sturm bei dem schon etwas exponierter gelegenen Gjedscr Rev und bei ENE-Sturm bei dem nach dieser Seite ganz freien Schultz Grund. Dreht der Sturm noch weiter in östliche Richtung, so kommt der Auslauf zum Durchbruch, der bei allen vier Feuerschiffen zwischen NNE und E einsetzt, ungefähr überall dort, wo der Sturm keinen Einlauf mehr bringen kann.

Die Gebiete der beiden Sturmgruppen schließen zwischen $\mathrm{N}$ und $\mathrm{E}$ einander aus, aber nicht an der anderen Berührungsfläche. Der Auslauf hört nicht dort auf, wo der Einlauf einsetzt, sondern greift über ihn hinaus und deckt sein Gebiet von SSW bis SW bei Drogden, von S bis SW bei Schultz Grund, von SSW bis NW bei Gjedser Rev und von SSW bis NNW bei Lappe Grund. Es ist demnach bei allen Feuerschiffen die Möglichkeit der Sturmrichtung bei Auslauf größer als bei Einlauf, und da beim Auslauf gerade auch die SW- und W-Stürme in Frage kommen, liegt der SchluB nahe, daß mehr Stürme mit Auslauf als mit Einlauf herrschen. Dieses stimmt jedoch nicht mit den Tatsachen überein, sondern die Zahl der Stürme mit Einlauf überwiegt im Jahresmittel bedeutend, $71 \%$ und $66 \%$ ist ihr Anteil bei Schultz Grund und Drogden, und wenn er bei Lappe Grund und Gjedser Rev $59^{\circ} \%_{0}$ resp. $58^{\circ}{ }_{0}$ ausmacht, so rührt es daher, daß, allerdings nur bei Gjedser Rev, ein Teil der Stürme mit Einlauf auf die Übergänge verteilt ist. Auch in den einzelnen Mitteln der Jahreszeiten überwiegt der Einlauf oder hält dem Auslauf die Wage.

Da ein Übergewicht des Einlaufes nicht nur in der Zahl der Stürme, sondern auch in ihrer Länge zum Ausdruck kommen muB, so haben u ir bei den Stürmen mit Einlauf und Auslauf die entsprechende Dauer der jeweiligen Stromrichtung nach Wachen in Rechnung gebracht: Die Angabe der Gesamtdauer des Einlaufes oder Auslaufes als Folge des Sturmes war dagegen schon aus den angeführten Gründen nicht möglich. Auch bei der Länge der Stürme ergab sich, daß jene mit Einlauf denen mit Auslauf überlegen sind. Am wenigsten günstig stellt sich für sie noch das Verhältnis bei Giedser Rev, und doch steht es hier 
schon wie $56: 44$, um über $64: 36$ bei Drogden auf $67: 33$ sowohl bei Schultz Grund wie bei Lappe Grund zu steigen.

Uberwiegen somit zweifellos die Stürme mit Einlauf in der Zeit des Auftretens, so ist damit keineswegs festgestellt, daß sie auch den größten Anteil am hydrographischen Einfluß besitzen. Er äußert sich am augenfälligsten in der Stromstärke. Wir haben deshalb ihre Mittelwerte für die neunjährige Periode gleichfalls getrennt nach ansgehendem und eingehendem Strom für eine Sturmzeit berechnet. Dabei ergab sich bei Gjedser Røv ein gleicher Wert für Einlauf und Auslauf, 1,2 Sm in der Stunde, so daß wegen des Überwiegens der Dauer des Einlaufes dieser in der Tat auch mehr einlaufendes Wasser bringt, als, bei Stürmen mit Auslauf ausläuft. Ebenso verhält es sich bei Drogden und Schultz Grund, wo die Stärke des Einlaufes sogar größer ist als die des Auslaufes. Der entgegengesetzte Zustand ergab sich dagegen für Lappe Grund, wo der Auslauf mit sogar 2,1 Sm in der Stunde den Einlauf mit 1,8 Sm übertrifft. Multiplizieren wir die Geschwindigkeit mit der Zahl der Wachen, so erhalten wir Auslauf: Einlauf wie 4725:4334. Da hierin nicht alle Einläufe und Ausläufe bei Sturm enthalten sind, so habe ich jene für die Übergangsgebiete auch noch zusammengestellt und berechnet, wobei sich das Verhältnis vom gesamten Auslauf zum gesamten Einlauf bei Stürmen wie 36:64 stellte.

Auch für die einzelnen Jahreszeiten trifft das generelle Bild zu. Bei Schultz Grund überwiegen fast immer die Einlaufstärke und Einlaufdauer, das gleiche trifft für Drogden zu und gilt hinsichtlich der Einlaufdauer auch für Gjedser Rev. Die Stromstärke ist dagegen im Winter beim Einlauf um 0,2 Sin geringer als beim Auslauf, und da für diese Zeit nur 85 Wachen Einlauf 89 Wachen Auslauf gegenüberstehen, so ist für den Winter der hydrographische Effekt der Stürme beim Auslauf größer als beim Einlauf. Bei Lappe Grund ist dem Jahresmittel entsprechend Einlaufstärke geringer als die Auslaufstärke, umgekehrt hierzu aber die Auslaufdauer größer als die Einlaufdauer.

In dem jahreszeitlichen Wechsel der Stromstärke ergibt sich nur bei Lappe Grund und Drogden insofern ein roher gleichmäßiger Gang zwischen Einlauf- und Auslaufstärke, indem die Maxima und Minima zusammenfallen. Die Maxima der Stromdauer gehören bei den beiden Feuerschiffen derselben Jahreszeit an, während bei Schultz Grund und Gjedser Rev kein Parallelismus in diesen Erscheinungen besteht. Auch die Differenzen zwischen den Stromstärken und der Stromdauer verlaufen individuell, indem bei der Stromstärke Lappe Grund und Drog- 
den gerade entgegengesetzt zueinander stehen, die Differenzen der Stromdauer aber in ihrer Zu- und Abnahme miteinander harmonieren. Schultz Grund und Gjedser Rev bewegen sich auch nach dieser Seite abweichend voneinander wie auch von den beiden übrigen Stationen.

Die erhaltenen Resultate gelten für die Positionen der Feuerschiffe. Wir sind nicht berechtigt, sie in der erhaltenen Form auf den Querschnitt der Wasserstraßen, in denen sie liegen, zu übertragen. Soll es versucht werden, über die Bewegungen in diesen während einer Sturmperiode Rückschlüsse zu ziehen, so ist die jeweilige Lage der Beobachtungsstationen von dem Gesichtspunkt der Stromverteilung zu würdigen Betrachten wir sie nach dem Gesetz, das rechts Einlauf und rechts Auslauf geht, so liegen die Feuerschiffe Schultz Grund und Drogden an der Einlaufseite, Lappe Grund ziemlich in der Mitte des Stromes, doch fällt es bei Berücksichtigung der Morphologie des Bodens gleichfalls auf die Einlaufseite, Gjedser Rev an der Auslaufseite. Ist also bei den drei zuerst genannten Stationen bei Sturm Einlauf beobachtet, so kann gleichzeitig auf der anderen Seite des Großen Beltes und des Sundes sehr wohl ein Auslauf existiert haben. Beobachtungsmaterial hierüber ist mir für die Querschnitte bei den Feuerschiffen nicht bekannt. Hier stellt sich also eine Lücke ein, es vernotwendigt sich, zur weiteren Auswertung der regelmäBigen Beobachtungen auch bei Sturm Strombeobachtungen in Querschnitten anzustellen. Es dürfte genügen, wenn sie bei typischen Sturmlagen je einmal vorgenommen werden. Alsdann wird es möglich sein, die Werte des Einlaufs und Auslaufs bei Stürmen ziemlich genau zu berechnen. Jetzt müssen wir uns mit vorstehender Überschlagsrechnung begnügen.

Die Ursache des allgemeinen Überwiegens des Einlaufes über Auslauf bei Sturm geht zweifellos auf das Überwiegen westlicher Stürme im südwestlichen Ostseegebiet zurück. Es ist eine bekannte Erscheinung, daß sie in diesem Gebiet stets einen niedrigen Wasserstand zur Folge haben, der durch verstärktes Nachströmen ausgeglichen werden soll. Hierzu kommt, daß bei westlichen Winden aus der Nordsee Wasser ins Skagerrak und Kattegat getrieben wird, das bei solchen Wasserstandsverhältnissen einen willkommenen Ausgang durch die Belte und Sunde in die Ostsee findet. Wir lernen hier zum ersten Male eindringlich kennen, daß die hydrographischen Erscheinungen der Beltsee und des Sundes nicht aus sich selber verständlich sind, sondern nur durch Betrachtung des Umwassers genetisch gewürdigt werden können. Für den vorliegenden Fall sind wir leider gegenwärtig nicht in der Lage, die gegenseitige Abhängigkeit schärfer zahlenmäßig zu begründen, da 
Tabelle 1 a. Drogden,

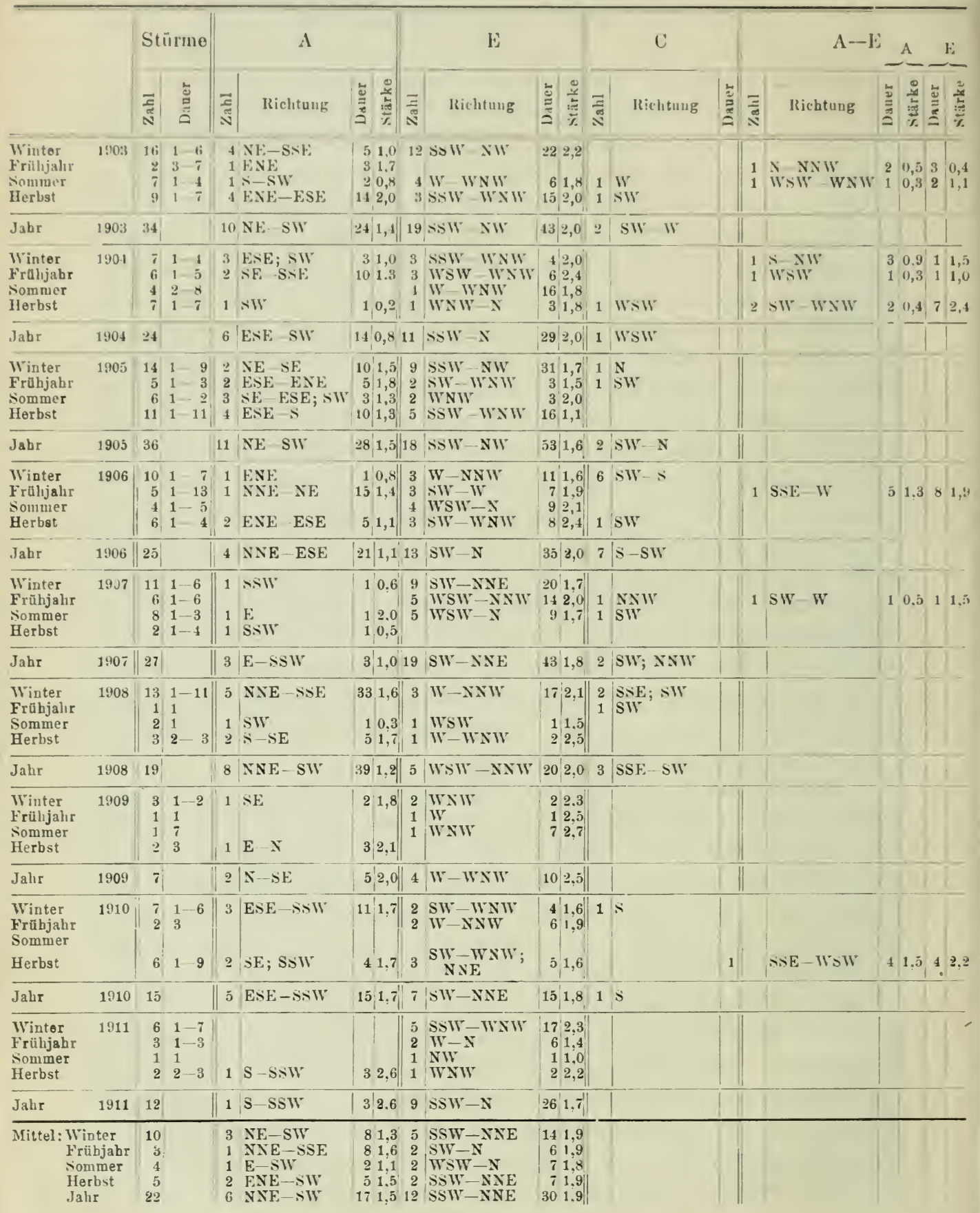


Sturm und Strom.

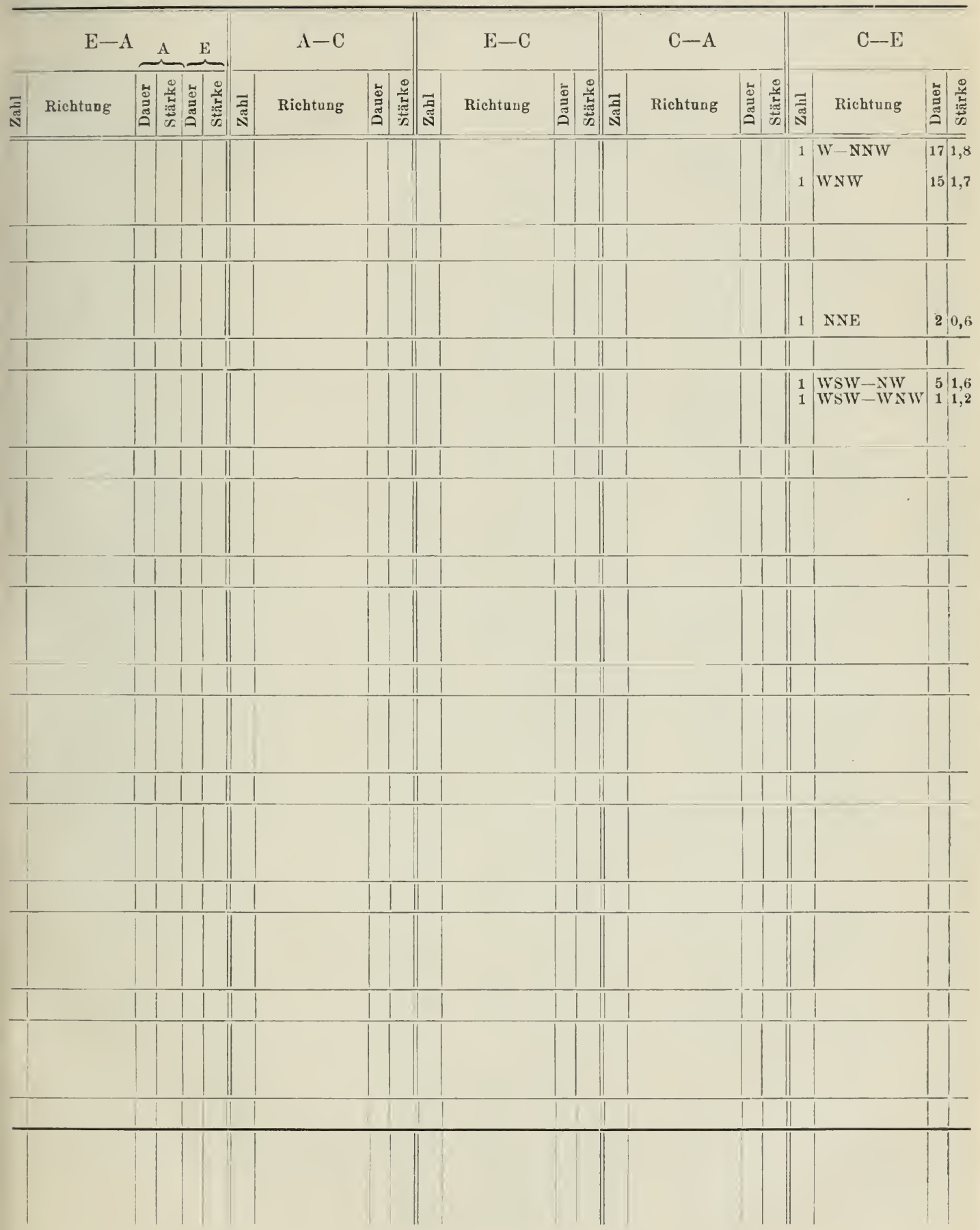


Tabelle 1e. Schultz Grund,

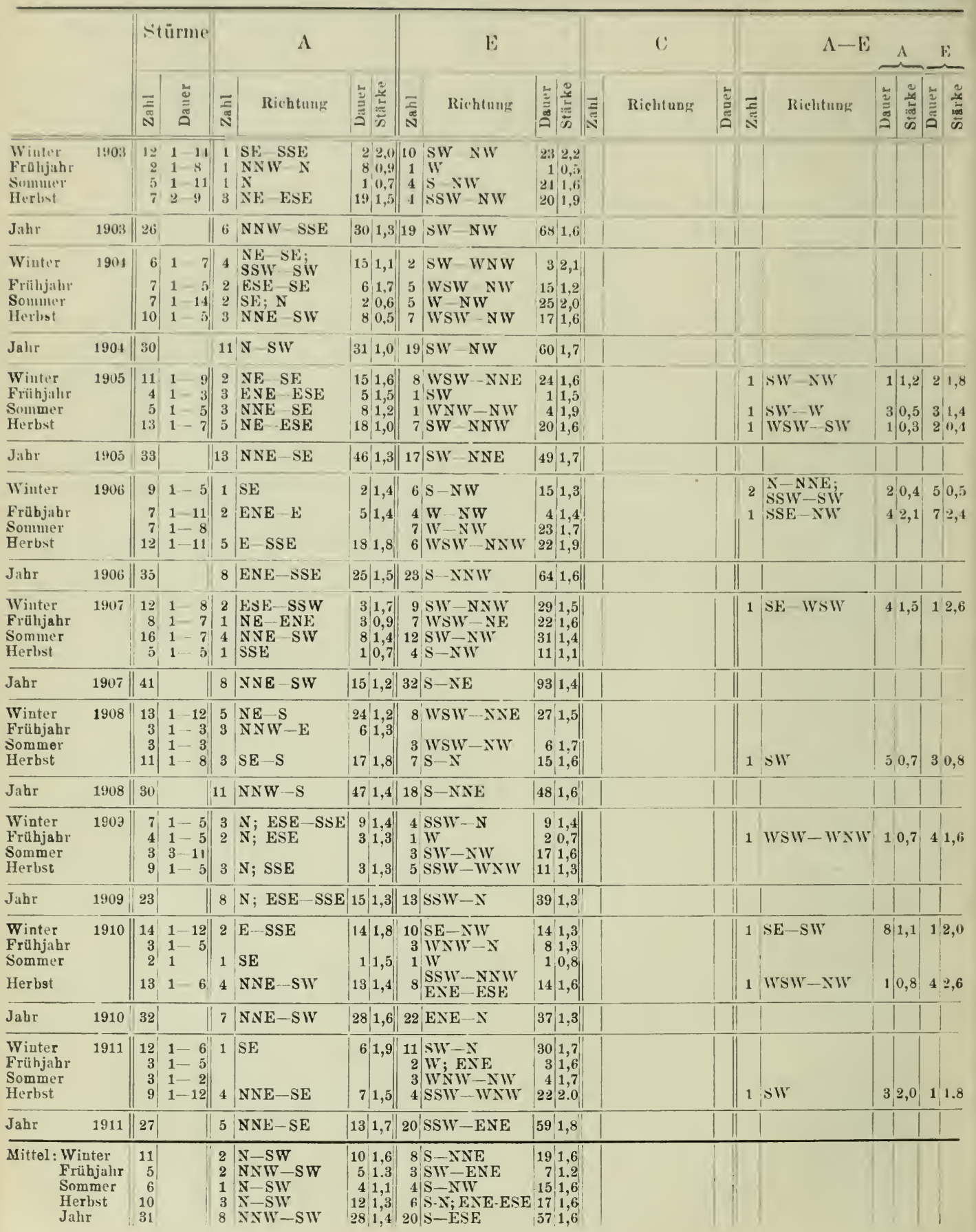


T'abelle 1d. Gjedser Rev.

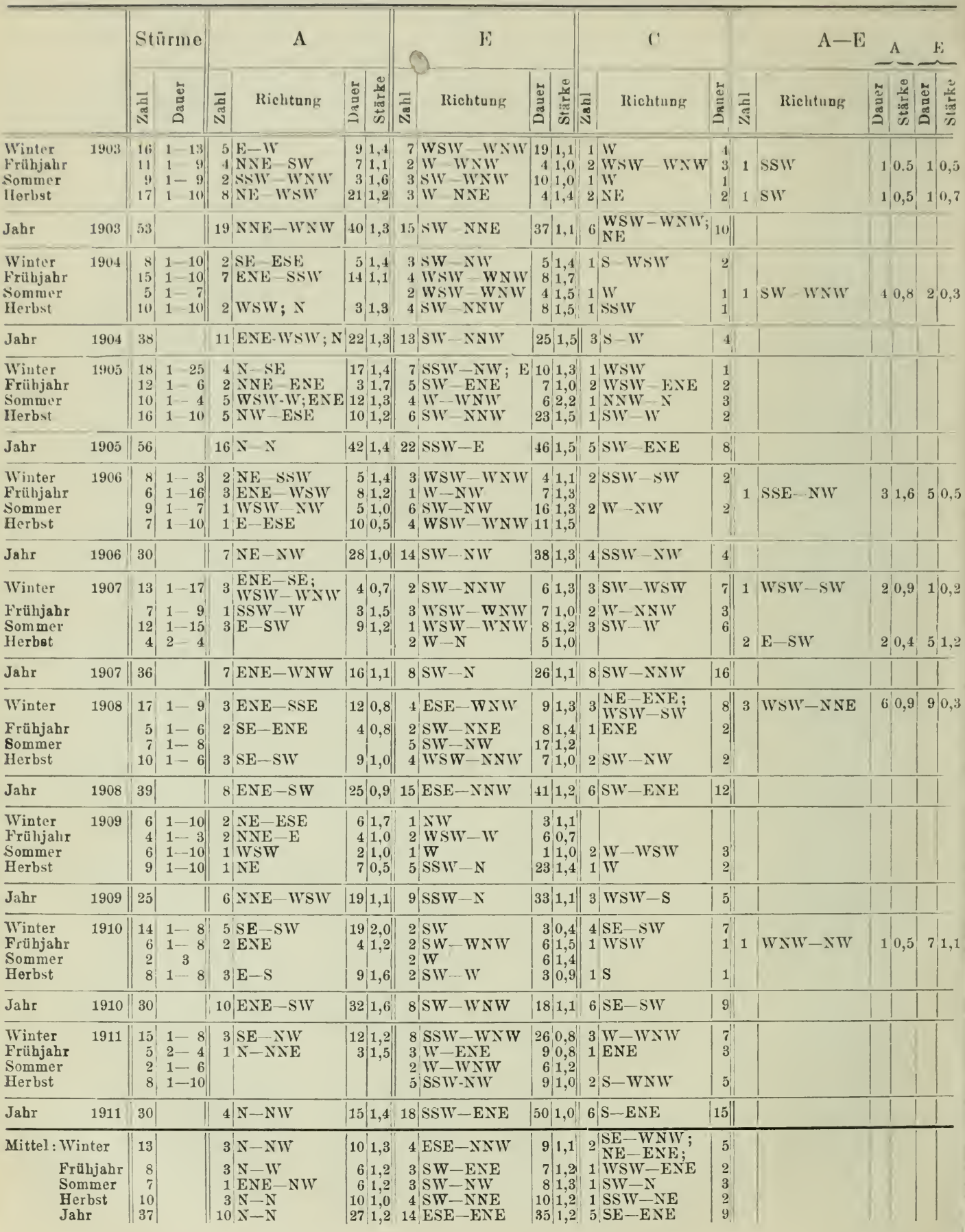


Sturm und Strom.

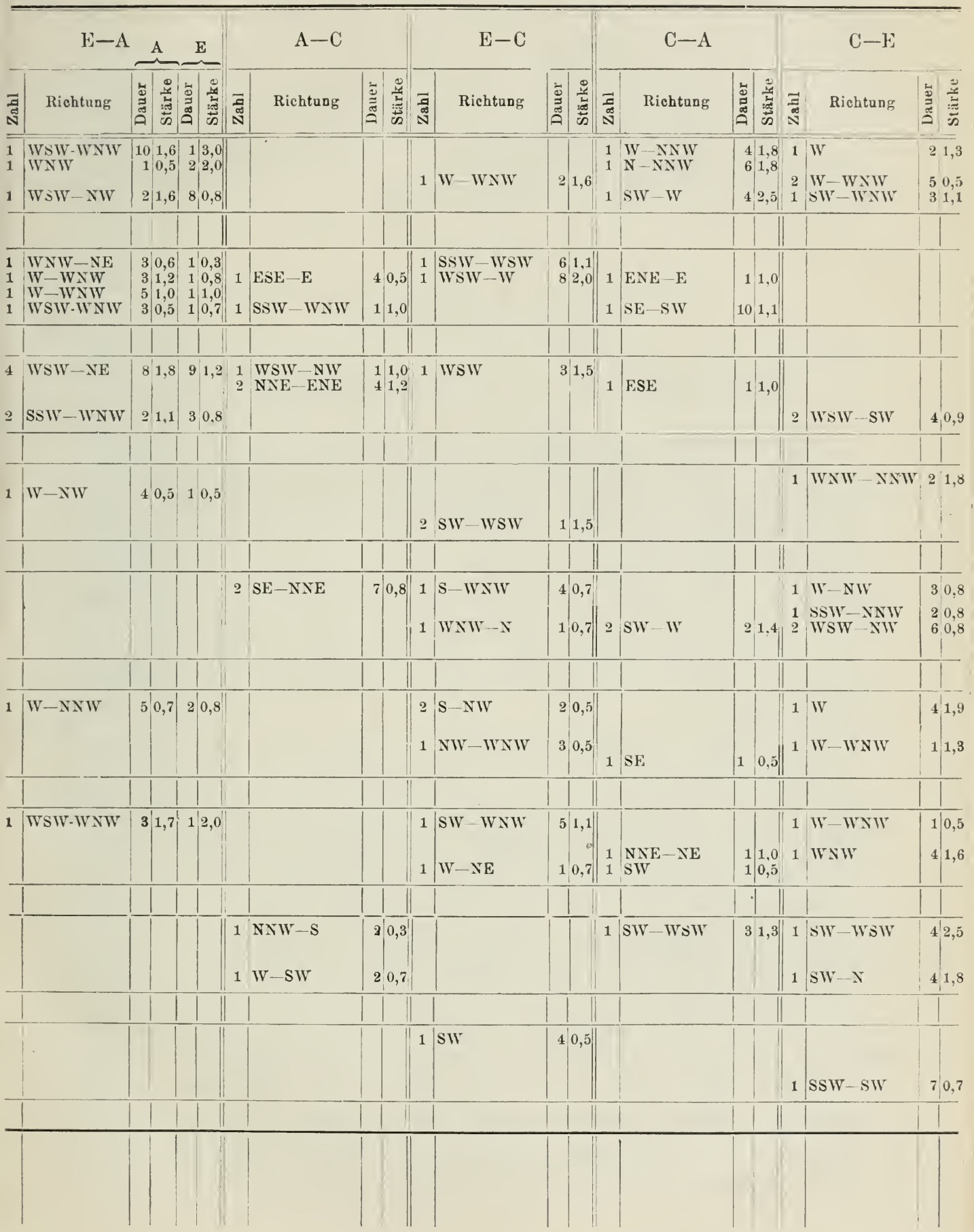


bei der vielseitigen Auswertung der Wasserstandsbeobachtungen in der südwestlichen Ostsee gerade die Beziehungen zwischen Sturm und Wasserstand nicht eingehender verfolgt wurden. Nur allgemein heißt es im Küstenstromwerk S. 751: Es besteht wohl kein Zweifel darüber, daß die mittleren Grenzwerte der Wasserstände vorzugsweise durch Richtung und Stärke der Winde in ihrer Verteilung über die Jahreszeiten bedingt werden. Die Betrachtung des gesanten Einlaufs und Auslaufs bringt uns hierüber die nötige Klarheit.

\section{Auslauf und Einlauf.}

Methodisches S. 32. - Die Monatsmittel S. 33. - Die Jahresmittel S. 34. - Die Wasserstandsschwankungen S. 35̃. - Die Stromgeschwindigkeit S. 37. - Der Wasseraustausch durch Sund und Stammbelt S. 39). - Tabellen S. 40.

In den Tabellen S. 40- 43 sind die Werte des Einlaufes, des Auslaufes, der Calmen und der Differenz Auslauf-Einlauf nach den Monatsmitteln, den Jahreszeiten und dem Jahresmittel für die einzelnen Jahre niedergelegt. Für diese Tabellen gilt mit Rücksicht auf die Vergleichbarkeit mit den Ergebnissen der Internationalen Meeresforschung wie für alle folgenden, daB für die .Jahresmittel die Zeit vom 1. Januar 1903 bis 31. Dezember 1911 zugrunde gelegt ist, für die Monatsmittel aber die Zeit vom 1. November 1902 bis 31. Oktober 1911. Die Jahreszeiten beginnen dementsprechend mit dem 1. Dezember 1902 und schließen mit dem 30. Norember 1911.

Die dänischen nautischen Jahrbücher veröffentlichen auf Grund der vierstündigen Beobachtungen bereits die monatlichen Prozentmittel. Für einige Monate, in denen wegen starken Eisganges die Feuerschiffe eingezogen waren und somit während einer Anzahl von Tagen die Beobachtungen ausfielen, habe ich unter Angabe der Zahlen für die Beobachtungstage die Mittel aus dem vorliegenden Material eingefügt. Ferner wurde die Differenz Auslauf-Einlauf für jeden Monat in die Tabelle eingestellt, ebenso die Calmen. Dann wurden die vier Mittelwerte für Auslauf, Einlauf, Auslauf-Einlauf und Calmen für jede Jahreszeit der neun Jahre berechnet, ebenso direkt aus den Monatsmitteln die vier Mittelwerte für die einzelnen Jahre. Unabhängig hiervon wurden die vier Mittelwerte der einzelnen Monate und Jahreszeiten für die neunjährige Periode aus den einzelnen Monats- resp. Jahreszeitenmitteln gewonnen. Da die Dezimalen nicht weiter berücksichtigt werden konnten, so ergeben sich infolge des Erhöhens bei den gesamten Monats- 
mitteln und Jahresmitteln mitunter Abweichungen um eine Einheit von Hundert, das gleiche gilt von der Differenz Auslauf--Einlauf.

Betrachten wir die Monatsmittel der korrespondierenden Beobachtungen auf Lappe Grund und auf Drogden, so zeigen alle für jeden Monat ein deutliches Überwiegen des Auslaufs über den Einlauf. Das Plus des Auslaufes schwankt bei Drogden zwischen 3 und $39 \%$ und bei Lappe Grund zwischen 12 und $53 \%$, so daß sich für das Jahresmittel der ersten Station eine Differenz von $22 \%$, für das der letzten eine solche ron $34 \%$ während der neunjährigen Periode ergibt.

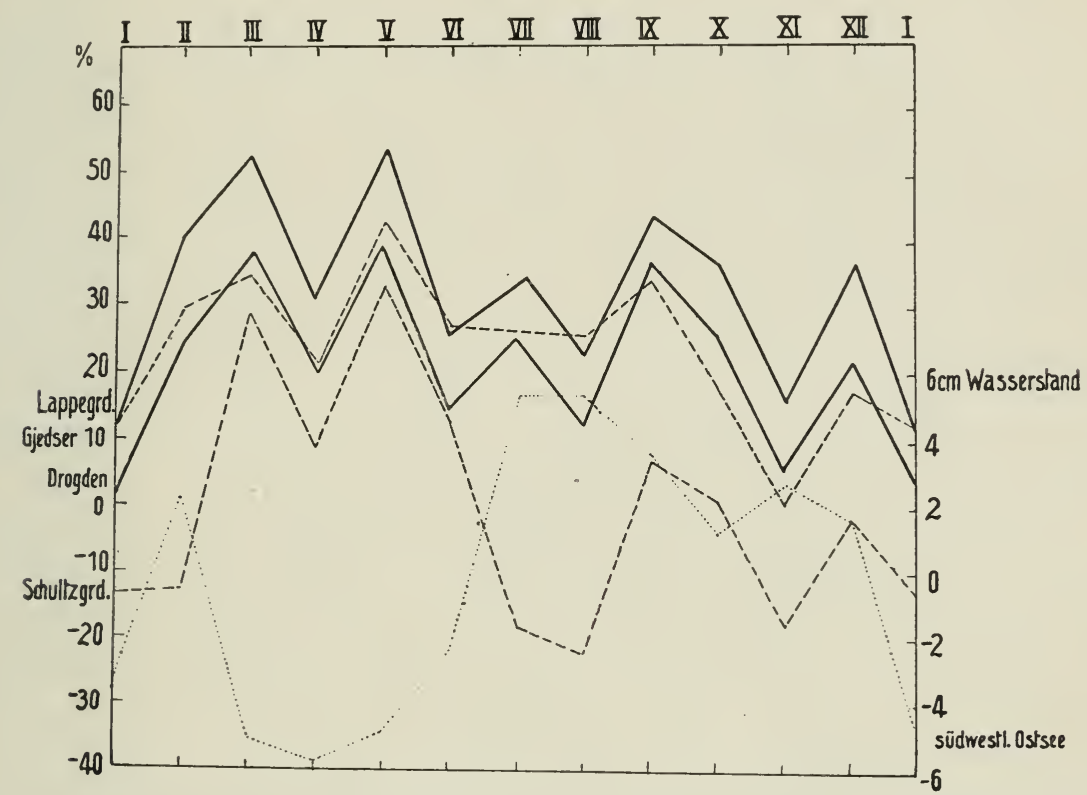

Fig. 1. Nonatsmittel der Differenz Auslauf-Einlauf vom 1. November 1902 bis 31. Oktober 1911 bei den Feuerschiffen Lappe Grund, Gjedser Rev, Drogden und Schultz Grund. Wasserstand der südwestlichen Ostsee von 1896-1905.

Die Schwankungen für die einzelnen Monate gehen auf beiden Stationen ganz und gar einander parallel. Eine deutliche Zunahme vom Februar bis Mai erleidet im April eine scharfe Unterbrechung. Zum Juni erfolgt eine ziemlich starke Abnahme, die nach einer leichten $\mathrm{Zu}-$ nahme im Juli bis zum August fortdauert. Einem stärkeren Steigen im September folgt dann eine Abnahme bis zum Januar, jedoch wiederum unterbrochen, und zwar durch eine ausgesprochene Zunahme im Dezember. Im Ganzen ergeben sich 5 Maxima, im März, Mai, Juli, September und Dezember. Ihnen stehen 5 Minima im Januar, April, Juni, 
August und November gegenüber. Alle Extreme sind ansgeprägt mit Ausnahme des .Julimaximums.

Der Parallelismus im C̈berwiegen des Auslaufes bei Lappe Grund und Drogden wiederholt sich zwischen Gjedser Rev und Schultz Grund. Maxima und Minima wechseln einander ab, bei Gjedser Rev um $4.2 \%$, bei Schultz Grund sogar um 55\% schwankend. Bei letzterem gibt es eine Anzahl Monate, in denen der Auslauf geringer ist als der Einlauf, im Maximum um 22" 2 im August, während das Maximum des Cberwiegens auf nur $33 \%$ im Mai kommt. Bei Gijedser Rev dagegen hat der Auslauf stets die Oberhand, wenn im November auch nur um $1{ }^{\circ}{ }_{0}$, so dafür im Mai mit $43 \%$ im Maximum. IDie Maxima und Minima sind auf Schultz Grund und Gjedser Rev denen der beiden Feuerschiffe des Sundes ähnlich mit Ausnahme des schon bei diesen nur schwach entwickelten Julimaximums. Es verschwindet, bei Schultz Grund tritt an seine Stelle sogar ein starkes Abnehmen. Ferner fällt bei dieser Station auf, daB das Anwachsen im Januar zwar auch vorhanden ist, aber außerordentlich gering, nur um $1 \%$. Alle vier Stationen besitzen ein gemeinsames Jahresmaximum im Mai, das das des März nur wenig übertrifft, dagegen kein gemeinsames Minimum. Dieses fällt bei den beiden Sundstationen auf den Januar, am Nordeıde des Großen Beltes dagegen auf August und bei Gjedser Rev auf November.

Stromstillen stellen sich bei allen Feuerschiffen ein. Bei Schultz Grund sind sie jedoch wie bei den Stürmen so selten, daß sie fast in allen Monatsmitteln für die einzelnen Jahre schon verschwinden und in den Monatsmitteln für die neunjährige Periode überhaupt nicht mehr zum Ausdruck kommen. Am stärksten sind sie bei Gjedser Rer, bei dem sie im Jahresmittel der Periode $26 \%$ ausmachen, während der gleiche Anteil bei Drogden und Lappe Grund auf $9 \%$ und $2 \%$ herabsinkt. Der hohe Prozentsatz bei Gjedser Rev hat seine Ursache in dem Gegensatz zwischen vorwaltendem Wind und vorherrschendem Strom. Der auslaufende Oststrom wird durch Westwinde zurückgehalten. Bei Drogden trifft dieses nur bei dem selteneren Nordwind ein, während die Nordausgänge von Sund und Großem Belt Stromstillen meistens nicht bei Rückstau, sondern beim Kentern des Stromes besitzen.

Im Jahresmittel der neunjährigen Periode zeigt sich deutlich der geringe Auslauf bei Schultz Grund. Er ist gleich Null, Auslauf und Einlauf stehen sich mit $50^{\prime \prime} / \%$ einander gegenüber. Bei Gjedser Rev beträgt dagegen im Jahresmittel das Uberwifgen des Auslaufes $24 \%$, bei Drogden $22^{\circ}$, bei Lappe Grund $34^{\%} \%$, mit anderen Worten, von 1903-1911 ist bei Lappe Grund 12\% mehr Auslauf-Einlauf als 
bei Drogden festgestellt worden, umgekehrt dagegen bei Schultz Grund $24^{\circ} \%$ weniger als bei Gjedser Rev.

Der Verlauf von Jahr zu Jahr zeigt in der neunjährigen Periode einen konformen Gang. Die Jahre 1903 und 1910 sind für den Sund ausgesprochene Maxima, 1904-1905 sowie 1911 sind Tiefstände. Von 1903-1205 und ron 1909-1911 zeigen auch Gjedser Rev und Schultz Grund denselben Verlauf, für die Zwischenzeit dagegen recht erhebliche Abweichungen.

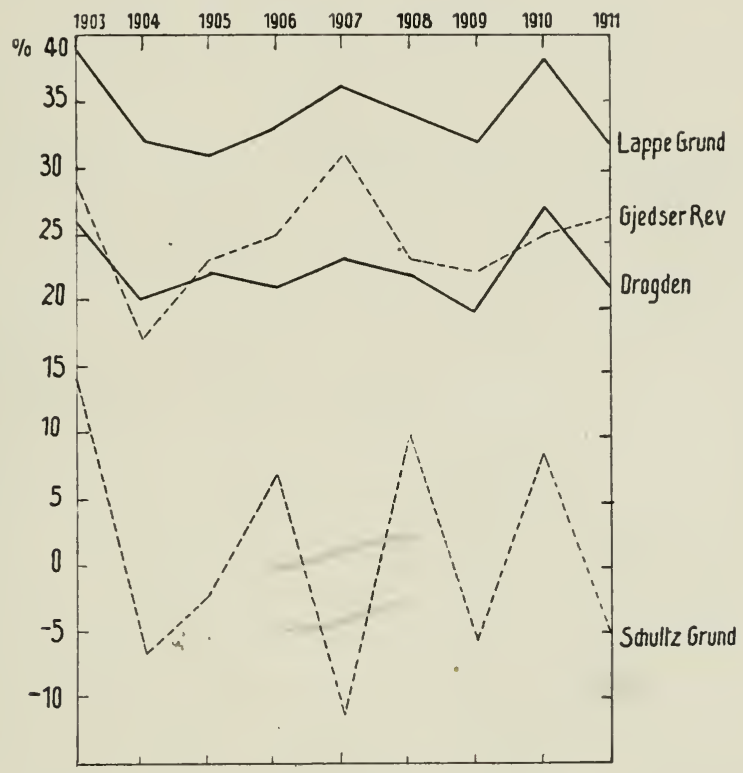

Fig. 2. Jahresmittel der Differenz Ausfluß - Einfluß bei den Feuerschiffen Lappe Grund, Giedser Rer, Drogden und Schultz Grund. 1. Januar 1903 bis 31. Dezember 1911.

Forschen wir nach der Ursache der jährlichen Schwankungen zwischen Auslauf und Einlauf, so weist der ausgesprochen ähnliche Gang zwischen den vier Feuerschiffen auf eine gemeinsame Ursache hin; da die Extreme gleichzeitig eintreten, leider nicht auf die Richtung ihrer Herkunft. Es liegt nahe, ihre Ausbildung in den jährlichen Wasserstandsschẃankungen der südwestlichen Ostsee zu suchen, indem ein hoher Wasserstand hierselbst einen Auslauf, ein niedriger einen Einlauf zur Folge hat, wie wir es für den letzten Fall schon bei den Stürmen kennen lernten. Allein es besteht hier kein durchgehender Parallelismus in dem Auftreten der Erscheinungen. Wir stellen nach dem Küstenstromwerk Seite 753 die dort gegebenen Mittel der monatlichen durch- 
schnittlichen Abweichungen für Vorpommern, Mecklenburg und Holstein zusammen, denen fast überall die Zeit 1896 - 1905 zugrunde gelegt ist, und fügen das Gesamtmittel hin\%u.

\begin{tabular}{|c|c|c|c|c|c|c|c|c|c|c|c|c|}
\hline & 1 & II & III & IV & V & VI & VII & VIII & IX & $X$ & XI & XII \\
\hline Vorpommern & -3 & +3 & -7 & -7 & -6 & -3 & +6 & +7 & +5 & +1 & +2 & +2 \\
\hline Mecklenburg & -3 & +1 & $-\check{\jmath}$ & -6 & -4 & -2 & +5 & +5 & +3 & +2 & +2 & +2 \\
\hline Holstein . & -4 & +3 & -3 & -5 & -4 & -2 & +5 & +4 & +3 & +1 & $1+-1$ & +1 \\
\hline
\end{tabular}

. Iittel $(\mathrm{cm}) \cdot \mid-3,3+2,3-5,0-6,0-4,7-2,3+5,3+5,3+3,7+1,3+1,7+1,7$

Es fällt ein deutliches Minimum in den April, ein ebenso deutliches Maximum in den Juli und August. Neben diesen Extremen bringen November und Dezember ein sekundäres Maximum und der Januar ein sekundäres Minimum. Wir haben die Kurve jenen über die monatlichen Differenzen zwischen Auslauf und Einlauf eingefügt (Fig. 1, S. 33). In der zweiten Jahreshälfte geht sie im großen und ganzen um je einen Monat der Kurve der Differenzen voraus, auch das Maximum der letzteren wird durch sie im Februar wiedergegeben, so daß sie zur Erklärung herangezogen werden kann. Dieses Ergebnis bleibt auch bestehen, wenn wir die Wasserstandsschwankungen an den südschwedischen und dänischen Küsten ins Auge fassen. Petterssons Zusammenstellungen haben gezeigt, wie an den Küsten Südschwedens das Hauptmaximum auf November oder Oktober fällt, ein sekundäres auf den Dezember, und Paulsens Diskussion zeigt, wie in den Gewässern der dänischen Inseln das Maximum in September, im Kattegat teilweise aber erst im Oktober auftritt. ${ }^{1}$ )

Allein einem Zusammenhang zwischen Auslauf und Wasserstandss:hwankungen steht ein Bedenken gegenübcr. Ein höherer Wasserstand der südwestlichen Ostsee kann sich nur dann in Auslauf verwandeln, wenn in Skagerrak und weiterhin in der Nordsee sich ein niedrigerer Wasserstand vorfindet. Wir haben auf Grund von Paulsens Berechnungen die monatlichen Differenzen des Wasserstandes zwischen Frederikshavn und Gjedser gebildet, indem wir die Differenz vom Mittelwasser der beiden Positionen in Anschlag brachten. Es sei ausdrücklich betont, daß Paulsens Reihe von 1893 bis 1902 läuft, jedoch dürfte sie lang genug sein, um die regelmäßigen Züge zum Ausdruck zu bringen. Das Ergebnis der Differenzen Gjedser-Frederikshavn ist in $\mathrm{cm}$ folgendes:

1) A. Pauls en, Die dānischen Wasserstandsmessungen und einige Resultate derselben mit Bezug auf den Einfluß des Windes auf die Höhe des Wasserstandes. Meteorol. Zeitschrift, Hannband, Braunschweig 1906. 


\begin{tabular}{c|c|c|c|c|c|c|c|c|c|c|c}
\hline I & II & III & IV & V & VI & VII & VIII & IX & X & XI & XII \\
\hline 2,9 & 12,6 & 10,4 & 11,9 & 11,3 & 7,6 & 6,5 & 6,0 & 6,0 & 3,1 & 1.8 & $-0,9$
\end{tabular}

Vergleichen wir sie mit der Kurve über die Differenzen AuslaufEinlauf, so erkennen wir sofort vom Minimum des Dezember ab bis zum Mai den gleichen Verlauf, nur daß die Wasserstandsdifferenzen um einen Monat vorauslaufen. In dem anderen Jahresteil ist dagegen kein Parallelismus bemerkbar. Dieses dürfte seine Ursache darin haben, daß die Differenzen zwischen Gjedser und Frederikshavn während dieser Zeit bedeutend geringer sind und deshalb nicht so durchgreifend wirken.

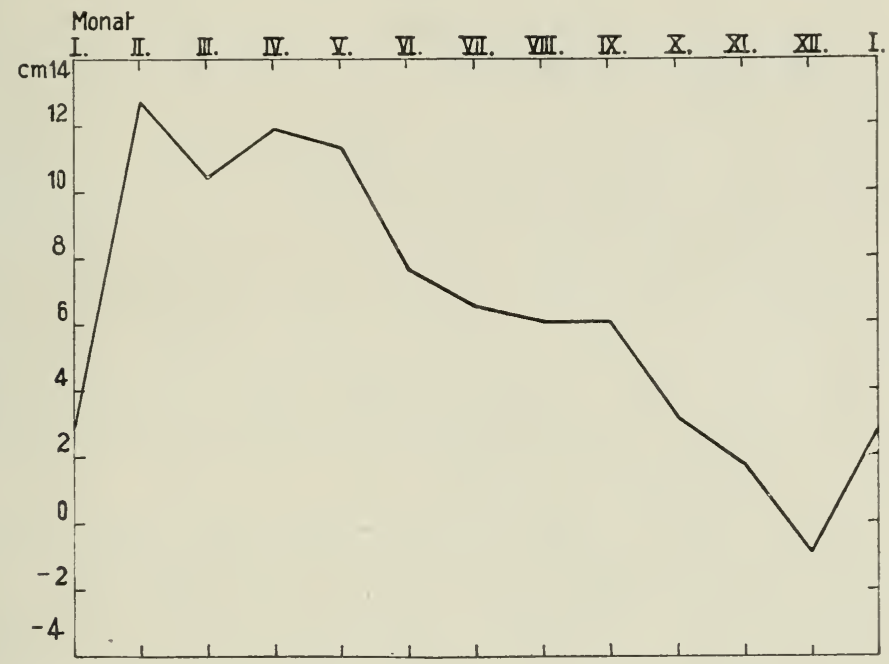

Fig. 3. Wasserstandsdifferenz Gjedser-Frederiksharn 1893-1903.

In diesen Monaten wandert das sommerliche Maximum der südwestlichen Ostsee bis zum Ausgang des Kattegat und findet erst im Ozean seinen Ausgleich.

Um die hydrographische Bedeutung von Einlauf und Auslauf richtig abschätzen zu können, haben wir die mittlere Stromgeschwindigkeit für die einzelnen Feuerschiffe berechnet. Das nautische Jahrbuch veröffentlicht bereits für die einzelnen Jahre die Monatsmittel, aus denen wir die Mittel für die einzelnen Jahre der neunjährigen Periode wie auch die Mittel der einzelnen Monate für die gleiche Zeit gewonnen haben. Der März 1909 ist hierbei mangelnder Bcobachtungen halber nicht verwertet; wir haben ihn nicht interpoliert, da ja gerade abnormer Zustände wegen, ungewöhnlich starken Eisganges halber, die Feuer- 
schiffe eingezogen werden muBten. Hingegen habe ich den Februar 1909 auf Station Lappe Grund, den das nautische Jahrbuch auch nicht herücksichtigt hat, doch mit in die Rechnung eingeführt, da die Beobachtumgen nur von vier Tagen fehlen.

Allgemein zeigt sich, daß die Monatsmittel der Stromgeschwindigkeit nur sehr wenig vom Jahresmittel abweichen. Es ergibt sich für den Sommer ein Nachlassen, das bei Drogden sein Minimum schon im Juni erreicht, bei Schult\% Grund in Juli, während es sich bei den beiden übrigen Feuerschiffen auf mehrere Monate erstreckt, von Juni bis August bei Latppe Grund und von April bis August bei Gijedser. Die Zeit des regsten Wasseraustausches ist bei Drogden der. Januar, ebenso bei Schultz Grund, nur daß sich bei ihm noch im März und November zwei gleich hohe Maxima einstellen, die bei Lappe Grund vier zwischen Oktober und März deutlich übertreffen. Bei Gjedser Rev ist ein Maximum nicht an einzelne Monate geknüpft, sondern erstreckt sich ron Januar bis März, während sich ein zweites auf den September beschränkt. Im Jahresmittel zeigt sich, daß bei Lappe Grund die lebhaftesten Strömungen herrschen, 0,3 Knoten in der Stunde mehr als bei Drogden. Schultz Grund übertrifft ebenso Gjedser Rev mit einem Plus von 0,4 , doch bleibt es um 0,3 gegen Lappe Grund zurück. Die im allgemeinen größere Geschwindigkeit des Sundes dürfte ihre Ursache in der Enge des Fahrwassers haben.

Die Schwankungen der gesamten Stromgeschwindigkeit gelten auch für Einlauf und Auslauf getrennt, die wir für Gjedser Rev und Lappe Grund berechnet haben. Die Abweichungen vom Mittel sind natürlich nur gering, das gleiche gilt für die Änderungen von Jahr zu Jahr, die hier in den Abweichungen rom neunịahrigen Mittel wiedergegeben seien.

\begin{tabular}{|c|c|c|c|c|c|c|c|c|c|c|c|}
\hline & & & 1903 & 1904 & 1905 & 1906 & 1907 & 1908 & 1909 & 1910 & 1911 \\
\hline & . & & $+0,3$ & & & & & & 0,0 & & \\
\hline gden & . & & 0.0 & + & 0 & $+0,1$ & 0.0 & $+0,1$ & $+0,1$ & 0,0 & $+0,2$ \\
\hline Grund & . & & $+0,1$ & $+0,1$ & $+0,1$ & +0.1 & 0,0 & 0,0 & 0.0 & $-0,1$ & 0,0 \\
\hline Gjedser Rev . & . & & 0,0 & 0,0 & $+0,1$ & 0,0 & $-0,1$ & 0,0 & 0,0 & 0,0 & $+0,1$ \\
\hline
\end{tabular}

Betrachtet man die monatliche Stromstärke mit den monatlichen Veränderungen des Auslaufes, so ergibt sich keine Ïhnlichkeit im Verlauf. Auch ein Vergleich mit den monatlichen Wasserstandsbewegungen fällt unbefriedigend aus; wohl ähneln die Kurven in dem gesamten Verlauf, aber sie sind gegenseitig um etwa 2-3 Monate verschoben: die größten und geringsten Stromgeschwindigkeiten folgen dem höchsten und niedrigsten Wasserstand nach. 
Multiplizieren wir bei den einzelnen Feuerschiffen die mittleren "Stromgeschwindigkeiten mit den Prozentzahlen für Einlauf und Auslauf, so erhalten wir unter der Voraussetzung, daß das Mittel der Stromgeschwindigkeiten sich gleichmäßig auf Einlauf und Auslauf verteilt, für den jährlichen Umsatz bei dem Produkt aus Zeitdauer (in Prozenten) und Geschwindigkeit (in Sm.) ein Uberwiegen des Auslaufes um 41,8 bei Lappe Grund, 20,8 bei Drogden, 12,0 bei Gjedser Rev und 0,0 bei Schultz Grund.

Wir können diese Zahlen noch schärfer fassen. Bei Lappe Grund und Gjedser Rev vermögen wir sie in Verbindung zu setzen mit dem Quantum Wassers, das infolge Süßwasserzufuhr zur Ostsee alljährlich seinen Ausweg aus dem Sund und der Arkonasee finden muß. Für Schulz Grund können wir es nicht, da wir keine Anhaltspunkte für eine Schätzung des Auslaufes durch den Kleinen Belt besitzen, für Drogden vermögen wir es nur ungenau, da die vorliegende Berechnung des Wasserhaushaltes der Ostsee den Sund, die Arkonasee und die Gotlandsee zusammengefaßt hat. ${ }^{1}$ ) Diese müssen an Zufuhr weniger Verdunstung einschließlich ihrer Zufuhrgebiete jährlich rund $510 \mathrm{ckm}$ Wasser hinausführen, ein Betrag, den wir mit $500 \mathrm{ckm}$ einkalkulieren wollen.

Ferner können wir das Verhältnis des Überwiegens des Auslaufens an beiden Stationen richtig beurteilen, indem wir die Auslaufgeschwindigkeit einfügen, die wir soeben für den monatlichen Gang schon besprochen haben. Ich habe sie bei Lappe Grund für die neunjährige Periode bedeutend größer als die Einlaufgeschwindigkeit gefunden, 1,4 gegenüber 0,9 , bei Gjedser Rev dagegen etwas kleiner, 0,7 gegenüber 0,8. Multiplizieren wir sie mit den Pruzentzahlen des Überwiegens vom Auslauf, so erhalten wir ein Verhältnis von Lappe Grund zu Gjedser Rev wie etwa $3: 1$.

Zu dem Auslauf der Süßwasserzufuhr gesellt sich der Auslauf eines Quantums Wasser, das ebenso groß wie der Einlauf ist. Für die Sundschwelle legen wir für 140 Tage im Jahr, an denen wir Einlauf fanden, den ganzen Querschnitt zugrunde; pflegt doch der Strom an der Oberfläche sich bei der geringen Tiefe meistens auf die ganze Wassermächtigkeit zu erstrecken. Wir erhalten bei einer angenommenen mittleren Geschwindigkeit ron $0,5 \mathrm{~km}$ in der Stunde, die eher zu niedrig als zu hoch gegriffen sein dürfte, $124 \mathrm{ckm}$ Einlauf. Für den Schnitt bei Gjedser Rev wollen wir bei Berücksichtigung des Bodenreliefs den

1) H. Spethmann, Der Wasserhaushalt der Ostsee. Zeitschr. Ges. f. Erdlsunde, Berlin 1912. 
'Tiablelle 2. Auslauf

Drogden

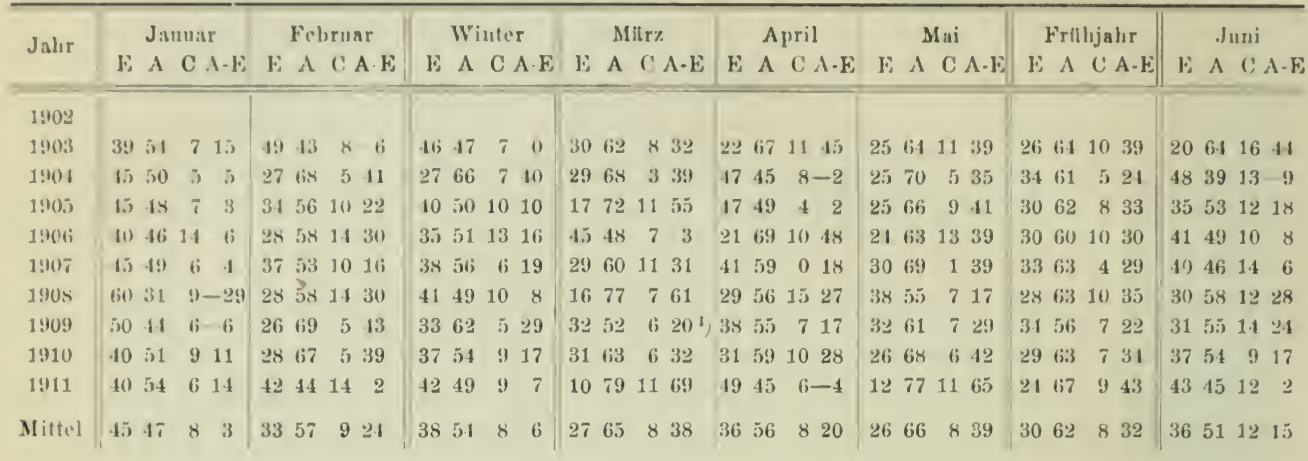

Lappe

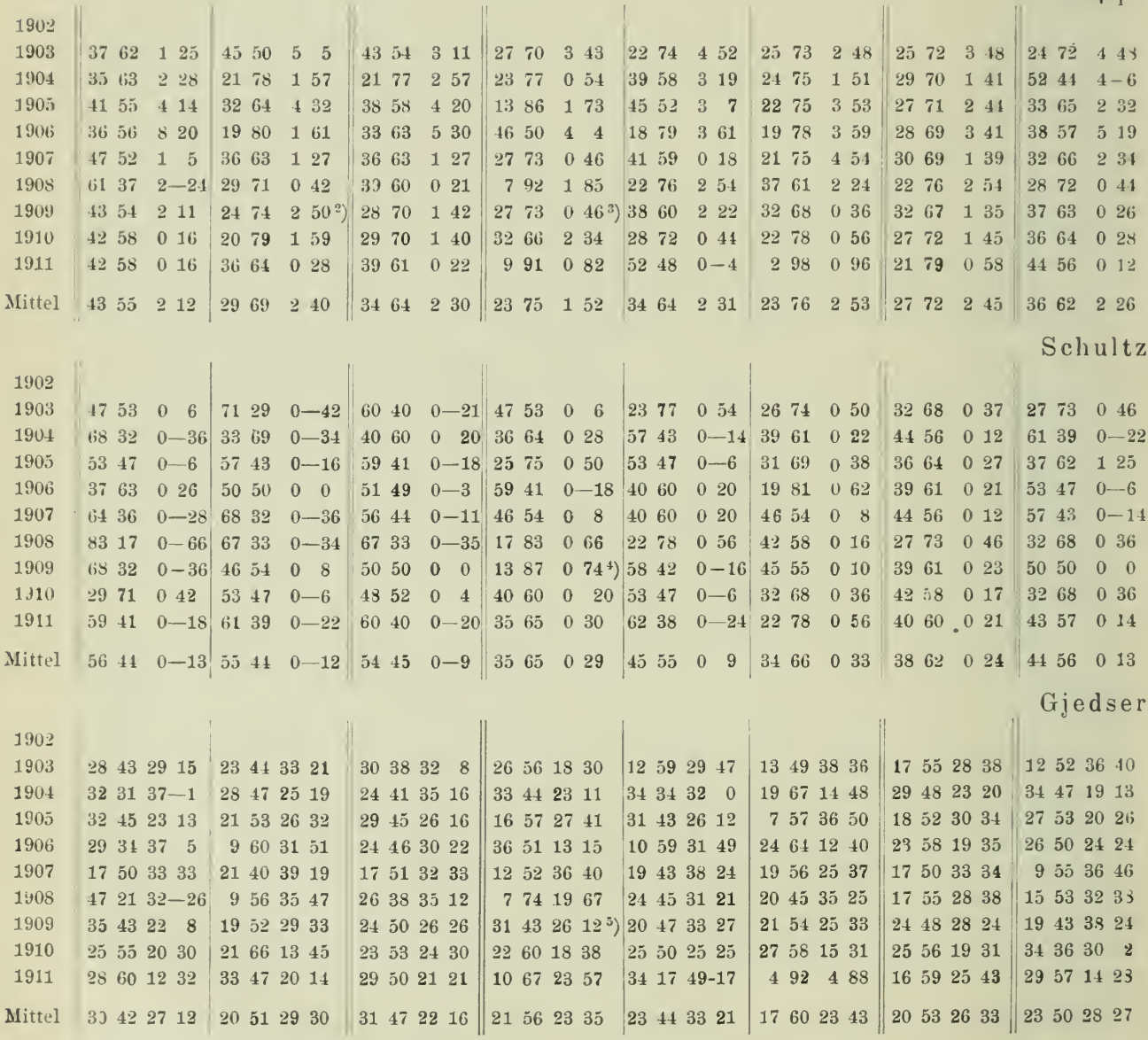

1) Nur 10 Tage beobachtet. 2) Nur an 24 Tagen beobachtet. 3) Nur an 19 Tagen heobachtet. 4) Nur an 9 Tagen 
und Einlauf in $\%$.

Drogden.

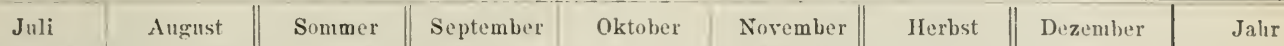

E A C A-E E A C A-E E A C A-E E A C A-E E A C A-E E A C A-E E A C A-E E A CA-E E E C CA-E

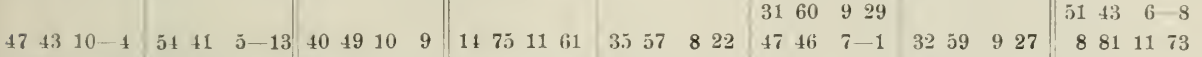

\begin{tabular}{llll|llll|lllll|llll|lllll|lllllll|llll}
23 & 63 & 14 & 10 & 35 & 45 & 17 & 13 & 35 & 50 & 15 & 15 & 21 & 71 & 8 & 50 & 39 & 57 & 4 & 18 & 42 & 47 & 11 & 5 & 34 & 58 & 8 & 24 & 11 & 47 & 12 & 6
\end{tabular}

\begin{tabular}{llll|llll|llll|llllllll|llllllll|llll}
33 & 51 & 11 & 13 & 28 & 55 & 17 & 27 & 34 & 53 & 13 & 19 & 30 & 66 & 4 & 36 & 36 & 53 & 11 & 17 & 29 & 52 & 19 & 23 & 32 & 57 & 11 & 25 & 38 & 50 & 12 & 12
\end{tabular}

\begin{tabular}{llll|llll|llll||llll|lllll|lllllll|llll|}
32 & 61 & 7 & 29 & 41 & 51 & 8 & 10 & 38 & 54 & 8 & 16 & 29 & 69 & $\mathbf{2}$ & 40 & 36 & 62 & 2 & 26 & 57 & 36 & 7 & -21 & 41 & 56 & 4 & 15 & 31 & 67 & 2 & 36
\end{tabular}

\begin{tabular}{llll|llll|llll|llll|llllllllllllllll|l}
25 & 62 & 13 & 57 & 45 & 47 & 8 & 2 & 37 & 52 & 12 & 32 & 35 & 56 & 9 & 21 & 25 & 63 & 12 & 38 & 35 & 53 & 12 & 20 & 32 & 57 & 11 & 26 & 35 & 58 & 7 & 23
\end{tabular}

\begin{tabular}{llll|llll|llll|llll|lllll|lllllllllll}
27 & 53 & 20 & 26 & 45 & 45 & 10 & 0 & 34 & 52 & 14 & 18 & 25 & 66 & 9 & 41 & 28 & 67 & 5 & 39 & 63 & 35 & 2 & -28 & 39 & 56 & 5 & 17 & 22 & 73 & 5 & 51
\end{tabular}

\begin{tabular}{lll|llll|llll|llll|lllllllll|llll|llll}
50 & 43 & $7-7$ & 29 & 65 & 6 & 36 & 37 & 54 & 9 & 18 & 18 & 77 & 5 & 59 & 53 & 44 & 3 & -9 & 34 & 53 & 13 & 19 & 35 & 58 & 7 & 23 & 44 & 44 & 12 & 0
\end{tabular}

\begin{tabular}{llll|llll|llll|lllll|lllllllll|llll|llll}
24 & 66 & 10 & 42 & 30 & 63 & 7 & 33 & 30 & 61 & 9 & 31 & 34 & 57 & 9 & 23 & 26 & 70 & 4 & 44 & 42 & 46 & 12 & 4 & 31 & 58 & 8 & 21 & 45 & 49 & 6 & 4
\end{tabular}

\begin{tabular}{llll|lll|llll|llll|llllllllllllllll}
31 & 63 & 6 & 32 & 49 & 47 & $4-2$ & 41 & 52 & 7 & 11 & 47 & 48 & 5 & 1 & 27 & 63 & 10 & 36 & 35 & 60 & 5 & 25 & 36 & 57 & 7 & 21 & 40 & 56 & 4 & 16
\end{tabular}

$33 \quad 58 \quad 9 \div 6$

$\begin{array}{llll}35 & 56 & 9 & 20\end{array}$

$\begin{array}{llll}34 & 56 & 11 & 23\end{array}$

$\begin{array}{llll}35 & 57 & 8 & 21\end{array}$

3.) $56 \quad 923$

$\begin{array}{llll}34 & 56 & 10 & 22\end{array}$

$\begin{array}{llll}36 & 55 & 8 & 19\end{array}$

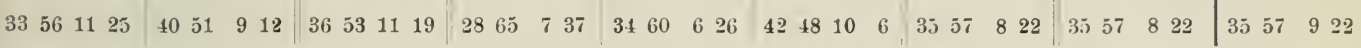
Grund.

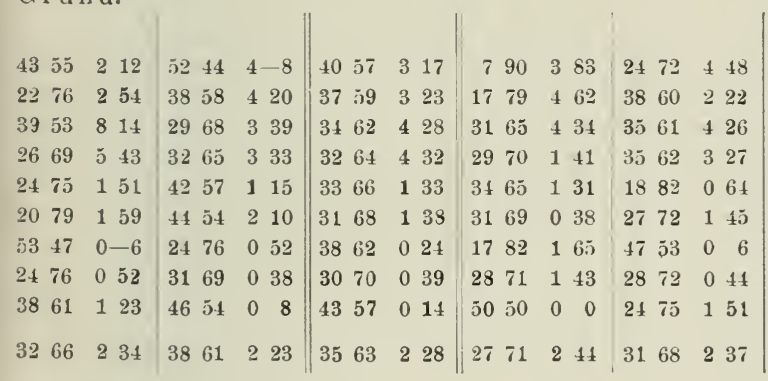

$\begin{array}{llll}26 & 72 & 2 & 46\end{array}$

$\begin{array}{llllllll}48 & 51 & 1 & 3 & 26 & 71 & 3 & 45\end{array}$ $4252 \quad 6 \quad 10 \quad 3264 \quad 3 \quad 31$ \begin{tabular}{llll|llll}
28 & 64 & 8 & 36 & 31 & 63 & 5 & 32
\end{tabular}

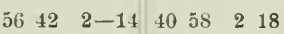
\begin{tabular}{llll|llll}
28 & 72 & 0 & 44 & 27 & 73 & 0 & 46
\end{tabular} $\begin{array}{llllllll}66 & 31 & 3-35 & 41 & 57 & 1 & 16\end{array}$ \begin{tabular}{llll|llll}
33 & 66 & 1 & 33 & 32 & 67 & 1 & 35
\end{tabular} \begin{tabular}{llll|llll}
33 & 59 & 3 & 21 & 31 & 67 & 1 & 36
\end{tabular} $\begin{array}{lllllllll}29 & 71 & 0 & 42 & 34 & 65 & 0 & 31\end{array}$ \begin{tabular}{|llll|llll}
41 & 57 & 3 & 16 & 33 & 65 & 2 & 32
\end{tabular}
$48 \quad 50 \quad 2 \quad 2$

$\begin{array}{llll}6 & 91 & 3 & 85\end{array}$ $4155+14$ $\begin{array}{llll}43 & 52 & 5 & 9\end{array}$ $\begin{array}{llll}26 & 74 & 0 & 48\end{array}$ $\begin{array}{llll}27 & 73 & 0 & 46\end{array}$ $\begin{array}{llll}17 & 83 & 0 & 66\end{array}$ $\begin{array}{llll}26 & 72 & 2 & 46\end{array}$ $\begin{array}{llll}39 & 60 & 1 & 21\end{array}$ $\begin{array}{llll}35 & 65 & 0 & 30\end{array}$ $\begin{array}{llll}30 & 68 & 2 & 37\end{array}$ $\begin{array}{llll}30 & 67 & 3 & 3\end{array}$ $\begin{array}{llll}33 & 65 & 3 & 32\end{array}$ $\begin{array}{llll}33 & 63 & 4 & 31\end{array}$ $\begin{array}{llll}32 & 65 & 3 & 33\end{array}$ $\begin{array}{llll}31 & 68 & 1 & 36\end{array}$ $\begin{array}{llll}32 & 66 & 1 & 31\end{array}$ $\begin{array}{llll}31 & 65 \quad 1 & 32\end{array}$ $\begin{array}{llll}31 & 69 & 1 & 33\end{array}$ $34 \quad 66 \quad 0 \quad 32$ $\begin{array}{llll}31 & 66 & 2 & 34\end{array}$

Grund.

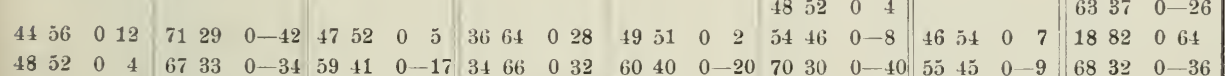
\begin{tabular}{llllllllllllllllllllllll|llll|llll}
85 & 15 & $0-70$ & 41 & 59 & 0 & 18 & 54 & 45 & $0-9$ & 58 & 42 & 0 & -16 & 56 & 44 & 0 & -12 & 48 & 52 & 0 & 4 & 54 & 46 & 0 & -8 & 67 & 33 & $0-34$
\end{tabular} \begin{tabular}{lllllllllllllllllllllllll|lllll}
52 & 47 & $1-5$ & 65 & 35 & $0-30$ & 57 & 43 & $0-14$ & 38 & 62 & 0 & 24 & 50 & 50 & 0 & 0 & 67 & 33 & 0 & -34 & 52 & 48 & 0 & -3 & 35 & 65 & 0 & 30
\end{tabular} 69 \begin{tabular}{lll|llll|lll|lllllllllllll|llll|llll}
61 & $0-38$ & 85 & 15 & $0-70$ & 70 & 30 & $0-41$ & 54 & 46 & $0-8$ & 29 & 71 & 0 & 42 & 58 & 42 & 0 & -16 & 47 & 53 & 0 & 6 & 52 & 48 & $0-4$
\end{tabular} \begin{tabular}{llllllll|llll|lllllllllllllllllll|llll}
31 & 69 & 0 & 38 & 57 & 43 & $0-14$ & 40 & 60 & 0 & 20 & 46 & 54 & 0 & 8 & 38 & 62 & 0 & 24 & 72 & 28 & 0 & -44 & 52 & 48 & 0 & -4 & 36 & 64 & 0 & 28
\end{tabular}

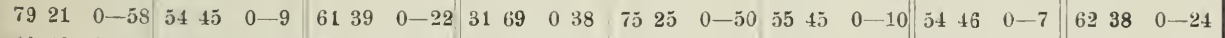
\begin{tabular}{lllllll|lll|lllllllllllll|llll|llll}
60 & 40 & $0-20$ & 44 & 56 & 0 & 12 & 45 & 55 & 0 & -9 & 49 & 51 & 0 & 2 & 43 & 57 & 0 & 14 & 58 & 42 & 0 & -16 & 50 & 50 & 0 & 0 & 60 & 40 & 0 & -30
\end{tabular} $\begin{array}{llllllllllllllllllllllllllllll}62 & 38 & 0-24 & 63 & 37 & 0-26 & 56 & 44 & 0-12 & 74 & 26 & 0-48 & 45 & 55 & 0 & 10 & 50 & 50 & 0 & 0 & 56 & 44 & 0 & -13 & 56 & 44 & 0 & -12\end{array}$ \begin{tabular}{|lll|lll|lll|llll|lllllllll||llll||llll|}
59 & 41 & $0-18$ & 61 & 39 & $0-22$ & 55 & 44 & $0-10$ & 46 & 53 & 0 & 7 & 50 & 51 & 0 & 1 & 59 & 41 & 0 & 18 & 52 & 48 & 0 & -3 & 51 & 49 & $0-2$ &
\end{tabular} $43 \quad 57 \quad 0 \quad 14$ $\begin{array}{llll}53 & 47 & 0-7\end{array}$ $\begin{array}{lll}51 & 49 & 0-2\end{array}$ $\begin{array}{llll}47 & 53 & 0 & 7\end{array}$ $\begin{array}{lll}56 & 44 & 0-11\end{array}$ $\begin{array}{llll}45 & 55 & 0 & 10\end{array}$ $53 \quad 47 \quad 0-6$ $\begin{array}{llll}46 & 54 & 0 & 8\end{array}$ $53 \quad 47 \quad 0-5$ Rev.

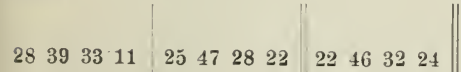
\begin{tabular}{lllll|llll|llll}
11 & 63 & 26 & 52 & 20 & 47 & 33 & 27 & 22 & 52 & 26 & 31
\end{tabular} \begin{tabular}{llll|llll|llll}
29 & 48 & 23 & 19 & 21 & 60 & 19 & 39 & 26 & 54 & 21 & 28
\end{tabular} \begin{tabular}{llll|llll|llll}
25 & 18 & 27 & 23 & 22 & 51 & 27 & 29 & 24 & 50 & 26 & 25
\end{tabular} \begin{tabular}{llll|llll|llll}
16 & 46 & 38 & 30 & 17 & 42 & 41 & 25 & 14 & 48 & 38 & 34
\end{tabular} \begin{tabular}{llllllll|llll}
13 & 47 & 40 & 34 & 35 & 40 & 25 & 5 & 21 & 47 & 32 & 26
\end{tabular} \begin{tabular}{lllllllll|llll}
33 & 37 & 30 & 4 & 12 & 62 & 26 & 50 & 21 & 47 & 31 & 26
\end{tabular}

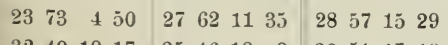

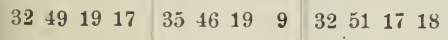
$23502727|24502526| 255027 \quad 27 \mid$ $\begin{array}{llll}18 & 52 & 30 & 34\end{array}$ \begin{tabular}{llllllll|llll||llll|llll}
2 & 73 & 25 & 71 & 27 & 36 & 37 & 9 & 26 & 37 & 37 & 11 & 18 & 49 & 33 & 31 & 13 & 44 & 43 & 31
\end{tabular} \begin{tabular}{lllllllll|llll||llll|lllll}
18 & 54 & 28 & 36 & 36 & 30 & 31 & -6 & 32 & 33 & 35 & 1 & 29 & 39 & 32 & 10 & 35 & 37 & 28 & 2
\end{tabular} \begin{tabular}{|llll|llll|llll||llll|llll|}
23 & 43 & 34 & 20 & 38 & 43 & 19 & 5 & 28 & 40 & 32 & 12 & 30 & 42 & 28 & 12 & 35 & 44 & 21 & 9
\end{tabular}

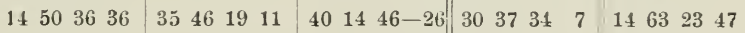
\begin{tabular}{llll|llll|llll|lllllllll}
17 & 67 & 16 & 50 & 6 & 64 & 30 & 58 & 38 & 39 & 23 & 1 & 20 & 57 & 23 & 36 & 23 & 39 & 39 & 15
\end{tabular} \begin{tabular}{llll|llll|lll|llllllll|l}
21 & 57 & 22 & 36 & 15 & 58 & 27 & 43 & 59 & 12 & 29 & -47 & 32 & 42 & 26 & 10 & 18 & 55 & 27 & 37
\end{tabular} \begin{tabular}{|llllllll|llll||llll|llll}
19 & 68 & 13 & 49 & 41 & 43 & 16 & 2 & 31 & 42 & 27 & 11 & 30 & 51 & 19 & 21 & 22 & 38 & 40 & 16
\end{tabular} \begin{tabular}{|lll|llll|llll|llll|llll|}
49 & 38 & $13-9$ & 32 & 58 & 10 & 26 & 34 & 46 & 20 & 8 & 38 & 47 & 14 & 8 & 26 & 44 & 30 & 18
\end{tabular} $\begin{array}{lll}33 & 58 & 95\end{array}$ 22562235
$3248 \quad 2016$

29472418 \begin{tabular}{lllll|lllll}
24 & 58 & 18 & 34 & 30 & 55 & 16 & 25
\end{tabular} \begin{tabular}{llll|llll}
34 & 35 & 31 & 1 & 28 & 46 & 26 & 18
\end{tabular}
50 $50 \quad 0 \quad 0$ $\begin{array}{lllll}20 & 48 & 32 & 29\end{array}$ $\begin{array}{llll}28 & 44 & 29 & 17\end{array}$ $\begin{array}{llll}26 & 49 & 25 & 23\end{array}$ $\begin{array}{llll}24 & 48 & 27 & 25\end{array}$ $\begin{array}{llll}18 & 49 & 33 & 31\end{array}$ $\begin{array}{llll}24 & 47 & 29 & 23\end{array}$ $\begin{array}{llll}25 & 48 & 27 & 22\end{array}$ 29541725 28541826 $\begin{array}{llll}25 & 49 \quad 26 & 24\end{array}$

bzobachtet. 5) Nur an 12 Tagen beobachtet. 


\begin{tabular}{|c|c|c|}
\hline & $\stackrel{=}{=}$ & 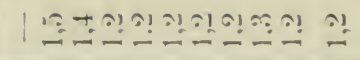 \\
\hline \multirow{13}{*}{ 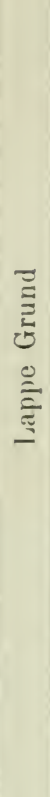 } & $\%$ & 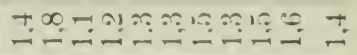 \\
\hline & $\therefore 0 N$ & \\
\hline & $1: 10$ & $1= \pm 010=0 !=0 \pm$ \\
\hline & Idas & 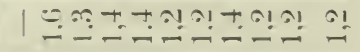 \\
\hline & $\sin$ & $1 \because \because \because 000=\because 00$ \\
\hline & ! & 1 $\because \because n=000=0$ \\
\hline & 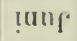 & 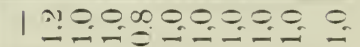 \\
\hline & אואי! & $100=01=00=$ \\
\hline & l!.udV & $1+m+=x_{0}=0,0 ?$ \\
\hline & 2.J? & 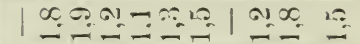 \\
\hline & यqक्ष & $19 \infty 0+\square$ \\
\hline & ued & $1010=0009 m$ \\
\hline & & 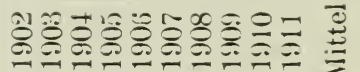 \\
\hline \multirow{14}{*}{ 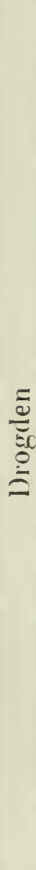 } & $\stackrel{\Xi}{\Xi}$ & 10000000000 \\
\hline & 'zodI & $=\because 0=\because=\because 0000$ \\
\hline & $\cdot 10 \mathrm{~N}$ & $\theta=0,0=0 x=\because \pm 0$ \\
\hline & 7.10 & $10,0 x+0,0=0$ \\
\hline & 7dəs & $1900=x 000=0$ \\
\hline & : & 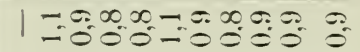 \\
\hline & $! n_{\Gamma} \cdot$ & $100 \pi=00000$ \\
\hline & țun & 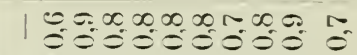 \\
\hline & !eIt & $1 \cong 00000=0 \Leftrightarrow 0$ \\
\hline & !!udF & 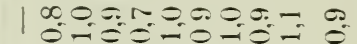 \\
\hline & ZIenT & 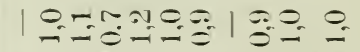 \\
\hline & iqक्ष & 1 $==0,00=0,01=$ \\
\hline & 'uer. & $1=\Xi 0100+0,001$ \\
\hline & & 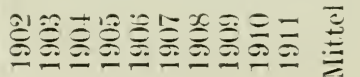 \\
\hline
\end{tabular}

\begin{tabular}{|c|c|c|}
\hline & $\cong$ & 1 $1=0000000$ \\
\hline & $\% 0(1$ & 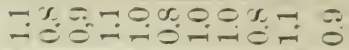 \\
\hline & N & $=00000000$ \\
\hline & . 190 & 190001000000 \\
\hline & flas & $1=0 \because=00 x+\infty 00$ \\
\hline & क्ञाIV & 1 I \\
\hline & !n! $\Gamma^{\circ}$ & 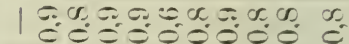 \\
\hline$=$ & 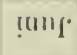 & | \\
\hline & !ִ & $10=0000000$ \\
\hline & {$\left[\begin{array}{ll}1.19 \\
Y\end{array}\right.$} & $100=100=10 \infty 0$ \\
\hline & ) & $1=\because \theta=\infty, 1000$ \\
\hline & $\therefore$ & 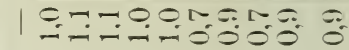 \\
\hline & 'UPe & $10,0100100=0$ \\
\hline & & 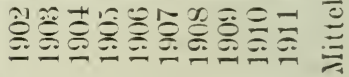 \\
\hline & $\stackrel{\Xi}{\Xi}$ & 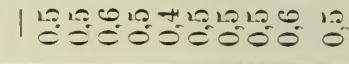 \\
\hline & $\cdot \mathrm{Ba}(\mathrm{I}$ & 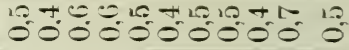 \\
\hline & $\triangle O N$ & 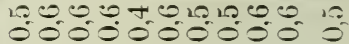 \\
\hline & $4: 10$ & 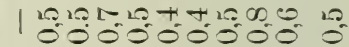 \\
\hline & zdos & | \\
\hline & बயम & 101001010100101010 \\
\hline & $!^{\mathrm{n}} \mathrm{\rho}$ & 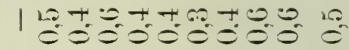 \\
\hline 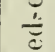 & !़ur & 1 a 000000000 \\
\hline & !ִ & $190000+10101010$ \\
\hline & I!.Idp & $10120+121212 t$ \\
\hline & Z.IEIT & 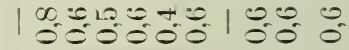 \\
\hline & •A㥩 & 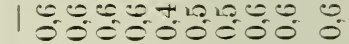 \\
\hline & uep & $1010+\operatorname{10} 1201250$ \\
\hline & & 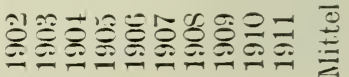 \\
\hline
\end{tabular}




\begin{tabular}{|c|c|c|}
\hline \multirow{14}{*}{ 节 } & $\stackrel{5}{\frac{\pi}{\pi}}$ & 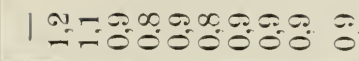 \\
\hline & " \%ә (I & 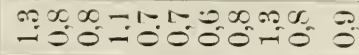 \\
\hline & $\cdot 10 \mathrm{~N}$ & $0+00=0=\infty=0=$ \\
\hline & 7.10 & $19+2000000=0$ \\
\hline & • 7 das & 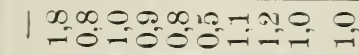 \\
\hline & siny & 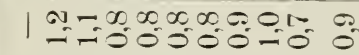 \\
\hline & $! n_{\Gamma^{*}}$ & 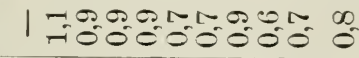 \\
\hline & IUIIי & 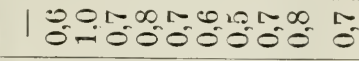 \\
\hline & एॉ & 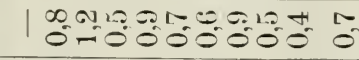 \\
\hline & {$\left[! . \mathrm{d} \mathrm{H}_{\mathrm{r}}\right.$} & 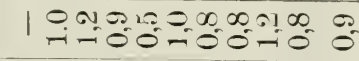 \\
\hline & ZIEIT & 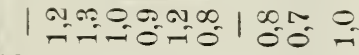 \\
\hline & $\mathrm{iq}^{4}$ & 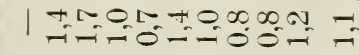 \\
\hline & 'ues & 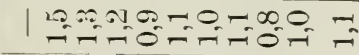 \\
\hline & & 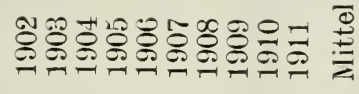 \\
\hline \multirow{14}{*}{ 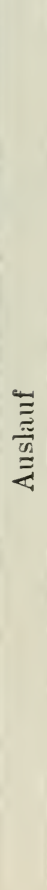 } & $\cong$ & 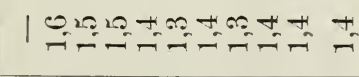 \\
\hline & 'zəd & 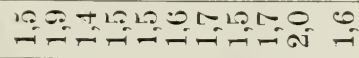 \\
\hline & $\cdot 10 \mathrm{~N}$ & 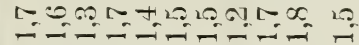 \\
\hline & 790 & 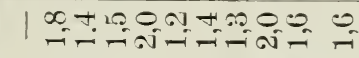 \\
\hline & '7dəs & $10,10 \Omega 0001020= \pm 10$ \\
\hline & : $811 \mathrm{Y}$ & 190000000000 \\
\hline & 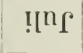 & $1+ \pm 0000=000$ \\
\hline & $\operatorname{lun} \Gamma^{\circ}$ & | \\
\hline & !以 & 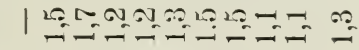 \\
\hline & I!Id & $10,0001200010+$ \\
\hline & Z.LEIT & 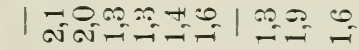 \\
\hline & 'دqәН & $1 \pm=1020+0.102000$ \\
\hline & 'uep & $100+20=01010$ \\
\hline & & 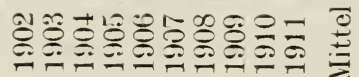 \\
\hline
\end{tabular}

\begin{tabular}{|c|c|c|}
\hline \multirow{14}{*}{$\frac{\stackrel{\Xi}{\Xi}}{\Xi}$} & ב & 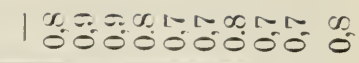 \\
\hline & "zə(I & $\begin{array}{l}0.1000000 n+\infty \\
-00000000\end{array}$ \\
\hline & $\triangle O N$ & 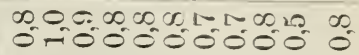 \\
\hline & 740 & 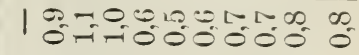 \\
\hline & $7 d \partial S$ & 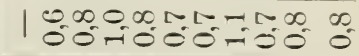 \\
\hline & $\cdot \stackrel{B n V}{ }$ & 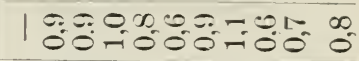 \\
\hline & $\varphi^{n} \rho^{\circ}$ & 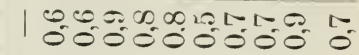 \\
\hline & !Uuم & $1=0.01001010=$ \\
\hline & אוני! & 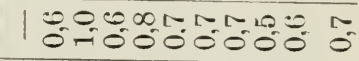 \\
\hline & I! $d^{d} \mathrm{~V}$ & 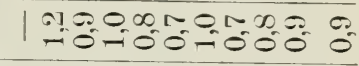 \\
\hline & ZIIII & 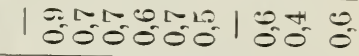 \\
\hline & - xqә & 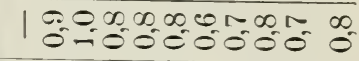 \\
\hline & 'Uе' & $1=0,0$ - $=0$ \\
\hline & & 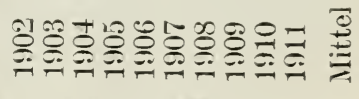 \\
\hline \multirow{14}{*}{ 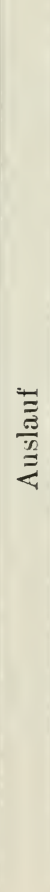 } & $\stackrel{\Xi}{\Xi}$ & 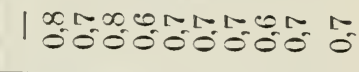 \\
\hline & 'zəब & 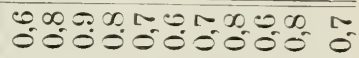 \\
\hline & ${ }^{\circ} \Lambda{ }^{\prime} \mathrm{N}$ & 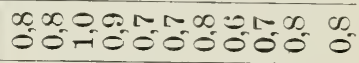 \\
\hline & 740 & $\begin{array}{l}\infty L \infty \in 00000 \\
00000\end{array}$ \\
\hline & zdas & 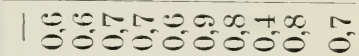 \\
\hline & : onv & 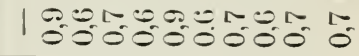 \\
\hline & $! \mathrm{n}_{\Gamma}$ & 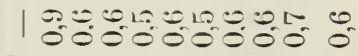 \\
\hline & !un & 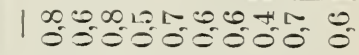 \\
\hline & איו"יו & 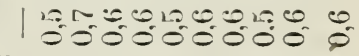 \\
\hline & {$[! .1 d \gamma$} & | \\
\hline & ZIEIT & 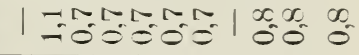 \\
\hline & :Iq & 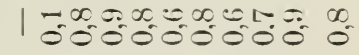 \\
\hline & 'ure & 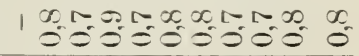 \\
\hline & & 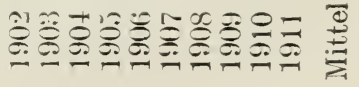 \\
\hline
\end{tabular}


Einlaufquerschnitt auf 0,05 q $k$ mm schätzen, $1 / s$ bis $1 / 9$ seiner ganzen Färhe. Nehmen wir als mittlere Einlaufgeschwindigkeit wiederum nur $0,5 \mathrm{~km}, 1$ so erhalten wir $219 \mathrm{ckm}$ pro Jahr. Als gesamter Einlauf ergeben sich also $343 \mathrm{ckm}$. Dieses ist cin scheinbar sehr hoher Wert gegenüber dem Auslaufquantum der Süßwasserzufuhr, doch zeigt ein vergleichender Úberschlag, daß man in der Tat mit einer solchen Größe zu rechnen hat. Lassen wir nämlich die 500 ckm Süßwasserzufuhr über beide Schwellen gleichmäßig auslaufen, ohne daß ein Einlauf geschieht, so würden sie beim Passieren in einem Jahre eine mittlere Auslaufgeschwindigkeit von $0,7 \mathrm{Sm}$. in der Stunde entfalten. Dieses stimnt zwar mit den Berechnungen für Gjedser Rev überein, bleibt aber stark hinter der Wirklichkeit bei Drogden zurück, wo der meisto Auslauf von statten geht und der Betrag 1,4 Sm. ausmacht. Führen wir den Wert des Einlaufes ein, so ergibt sich insgesamt ein Auslauf von $850 \mathrm{ckm}$ im Jahre, von dem entsprechend den gefundenen Verhältnissen rund $210 \mathrm{ckm}$ auf den Stammbelt und $640 \mathrm{ckm}$ auf den Sund kommen. Dieses ist natürlich nur ein rohes Ergebnis, es hat aber den Wert, eine ungefähre Vorstellung von den Wassermengen, die durch Sund und Belte zichen, zu wecken.

\section{Schwankungen des Salzgehaltes I.}

Drogden S. 44. - Lappe Grund S. 50. - Schultz Grund S. 52. - Gjedser IRev S. 54. - Fehmarnbelt S. 56. - Die monatlichen und die jährlichen Schwankungen S. 56. - Die Ursachen der Schwankungen S. 59. - Synoptisches Bild für die Schwankungen an den vier Feuerschiffen S. 61. - Tabellen S. 63.

Ein ruhiger, ungestörter Auslauf charakterisiert sich bei Drogden in einer sehr schön entwickelten, aber bei der geringen Tiefe der Station naturgemäß nicht sehr mächtigen Homohalinität. Die Differenz zwischen Oberfläche und $6 \mathrm{~m}$ resp. $8 \mathrm{~m}$ Tiefe beträgt nur wenige Zehntel Promille, für die großen Salzgehaltsschwankungen in der westlichen Ostsce ein sehr niedriger Wert. Ferner bewahrt der Salzgehalt eine ziemliche Konstanz, wie beispielsweise vom 13.-25. September 1906 und rom 19.-30. August 1909. Eine dritte Eigenschaft des gleichmäßigen Auslaufens ist ein verhältnismäBig niedriger Salzgehalt, der stets unter $10 \%$, in der Regel zwischen 7 und 8,5\% liegt. Der ruhige, gleichmäßig's Auslauf ließ sich besonders in dem windarmen April 1906 studieren. An einer Anzahl von Tagen war so gut wie gar keine Luftbewegung, wir greifen den 9.-11. April heraus. 


\begin{tabular}{|c|c|c|c|c|c|c|c|}
\hline & April 06 & $4 \mathrm{a}$ & $8 a$ & Mittag & $4 p$ & $8 p$ & Nitternacht \\
\hline \multirow[t]{3}{*}{ Wind } & 9. & C & C & SW 1 & C & C & C \\
\hline & 10. & C & C & WSII 2 & C & C & $\mathrm{C}$ \\
\hline & 11. & $\mathrm{C}$ & SW 1 & C & SSE 1 & $c^{\circ}$ & $\mathrm{C}$ \\
\hline \multirow[t]{4}{*}{ Strom } & 9. & A 0,3 & A 0,3 & A 0,3 & A 0,3 & A 0,3 & A 0,3 \\
\hline & 10. & A 0.3 & A $0, \tilde{5}$ & A 0,3 & A 0,5 & A 0,3 & A 0,3 \\
\hline & 11. & A 0,4 & A 0,3 & A 0,3 & A 0,5 & A 0,5 & A 0,5 \\
\hline & & $0 \mathrm{~m}$ & $3 \mathrm{~m}$ & $6 \mathrm{~m}$ & & & \\
\hline \multirow[t]{3}{*}{ Salz } & 9. & 7,8 & 7,8 & 7,8 & & & \\
\hline & 10. & 8,0 & 8,0 & 8,0 & & & \\
\hline & 11. & 7,8 & 7,8 & 7,8 & & & \\
\hline
\end{tabular}

Alle drei Kennzeichen sind leicht zu erklären. Die geringe Tiefe der Überlaufschwellen gestattet beim ruhigen Abfließen nicht den $\mathrm{Zu}$ tritt salzreichen Einlaufwassers; die Gleichmäßigkeit des Salzgehaltes geht auf das Ausströmen aus einem größeren Becken zurück, der niedrige Wert des Salzgehaltes auf die Landwärtslage des Zufuhrgebietes. Da dieses jährliche Schwankungen in seinem Salzgehalt ausführt, so müssen sie naturgemäß auch bei Drogden zum Ausdruck kommen. Wir werden hierauf bei der vergleichenden Betrachtung der monatlichen Veränderungen an den vier Feuerschiffen zurückkommen, dagegen wollen wir die Stürme, die Auslauf verursachen, schon jetzt würdigen.

In der Regel hat kurz vor oder nach ihnen Einlauf geherrscht. Ihre Wirkung macht sich alsdann in einer starken Verminderung des Salzgehaltes gegenüber dem vor kurzem oder gleich danach von Norden kommenden Wasser geltend. Namentlich gilt dieses für die Stürme aus östlicher Richtung, bei denen sich die gleichen Variationen in dem Verdrängen des Wassers in der Tiefe wie beim Einlauf an der Oberfläche einstellen, die wir sogleich bei Stürmen mit Einlauf näher würdigen werden.

Auslaufstürme schalten sich in einer längeren Periode ruhigen Auslaufens sehr selten ein. Das einzige klare, über die Fehlergrenze hinausgehende Beispiel, das ich gefunden habe, ist vom 20.-22. Oktober 1905.

\begin{tabular}{|c|c|c|c|c|c|c|c|}
\hline & Okt. 05 & $4 \mathrm{a}$ & $8 \mathrm{a}$ & Mittag & $4 \mathrm{p}$ & $8 \mathrm{p}$ & Mitternacht \\
\hline \multirow[t]{3}{*}{ Wind } & 20. & N 1 & NW 2 & WNW 1 & WSW 2 & SW 3 & SSW 5 \\
\hline & 21. & S 7 & S 6 & S 6 & SSE 5 & SSE 6 & SSE 4 \\
\hline & 22. & SSE 3 & ESE 3 & ESE 4 & $\mathrm{E} 4$ & $\mathrm{E} 4$ & ENE 4 \\
\hline \multirow[t]{3}{*}{ Strom } & 20. & A 1,0 & A 0,8 & A 1,5 & A 1,0 & A 1,0 & A 0,5 \\
\hline & 21. & A 0,5 & A 0,7 & A 0,7 & A 1,0 & A 1,3 & A 1,0 \\
\hline & 22. & A 1,0 & A 0,5 & A 1,3 & A 1,0 & A 0,7 & A 1,0 \\
\hline
\end{tabular}




\begin{tabular}{|c|c|c|c|c|}
\hline & ()lit. 0i) & () $\mathrm{mm}$ & $3 \mathrm{~m}$ & $6 \mathrm{~m}$ \\
\hline Saltz & $\begin{array}{l}2(1 . \\
21 . \\
22 .\end{array}$ & $\begin{array}{l}7,9 \\
7,7 \\
8,0\end{array}$ & $\begin{array}{l}8,1) \\
7,7 \\
8,11\end{array}$ & $\begin{array}{l}8,0 \\
7,7 \\
8,0\end{array}$ \\
\hline
\end{tabular}

Man erkennt als Effekt ein Herabdrücken des Salzgehaltes um ${ }^{3} / 10 \%$, der durch das Herantreiben des Oberflächenwassers der Arkonasee verursacht sein wird, nachdem es eine leichte Durchmischung in seinen obersten Lagen erhalten hat. Nach Nordoststürmen stellt sich mitunter auch ein Maximum ein. Am 30. November 1903, abends 8 Uhr und um Mitternacht herrschte bei Drogden ein Sturm aus ENE mit Stärke 9 und 8. Er brachte am nächsten Tage morgens 8 Uhr den auslaufendeu

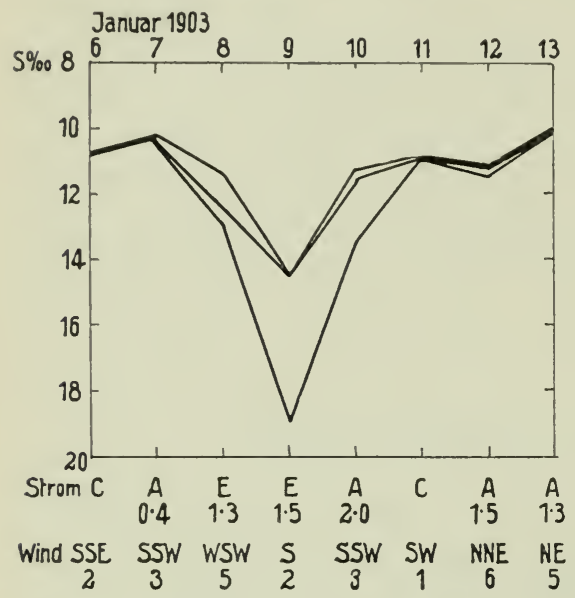

Fig. 4. Salzgehalt, Strom und Wind in $0 \mathrm{~m}, 3 \mathrm{~m}$ und $6 \mathrm{~m}$ Tiefe bei Feuerschiff Drogden vom 6.-13. Januar 1903. (Die oberste Kurve ist stets die von $0 \mathrm{~m}$, die unterste stets die von $6 \mathrm{~m}$ Tiefe.)

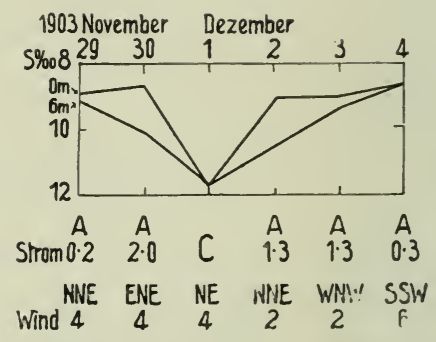

Fig. 5. Salzgehalt, Strom und Wind in $0 \mathrm{~m}$ und $6 \mathrm{~m}$ Tiefe beim Feuerschiff Drogden vom 29. November bis 4. Dezember 1903. (Die untere Kurve ist stets die von $6 \mathrm{~m}$ Tiefe.)

Strom zum Stehen, ohne aber einen Einlauf aufkommen zu lassen. Trotzdem stieg der Salzgehalt. Es scheint, daß aus der Tiefe etwas Wasser aus Norden nach Drogden gelangt ist. Durchmischtes Wasser aus Süden dürfte es deshalb nicht sein, weil der Salzgehalt schon am 'Tage vorher am Boden $\mathrm{zu}$ steigen anfing.

Für die Stürme mit Einlauf ist gleichfalls Homohalinität die Regel, aber mit bedeutend höherem Salzgehalt als bei Auslauf, woraus erhellt, daß der auslaufende Strom gänzlich ausgeschaltet wird. Es kann sich dieser Prozeß in verschiedenen Phasen abspielen. Zunächst das seltenere 
Vorkommnis, daß nicht Homohalinität beim einfließenden Wasser erreicht wird wie vom 6.- - J. Jalluar und vom 11.-13. Januar 1903. Am Morgen des 8. Januar tritt nach langsamem Kentern des Stromes bei WSW-Sturm, der nachmittags 4 Uhr Sturmstärke 7 erreicht, eine Zunahme des Salzgehaltes ein, verbunden mit einer Differenzierung, so daß am Morgen des 9. Januar zwischen Oberfläche und Boden $4,4^{\circ} \%$ Unterschied ist. Dieser gleicht sich langsam mit einlaufendem Strom innerhalb der nächsten beiden Tage wieder aus, so daß die Anschwellung des Salzgehaltes sich im großen und ganzen symmetrisch abspielt (Fig. 4 und 5). Einen unsymmetrischen Verlauf des gleichen Vorganges gibt der Anfang des November 1902, indern die Zunahme des Salzgehaltes als Folge eines Sturmes aus WNW sofort sich zwischen Boden und Oberfläche durchgehends bemerkbar machte, die Abnahme in der Tiefe sich aber um einen Tag verzögerte (Fig. 6).

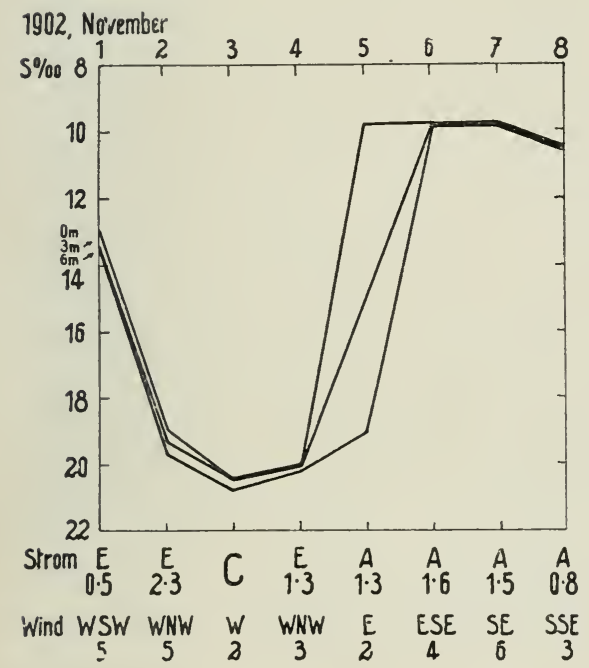

Fig. 6. Salzge!nalt, Strom und Wind in $0 \mathrm{~m}, 3 \mathrm{~m}$ und $6 \mathrm{~m}$ Tiefe bei Feuerschiff Drogden rom 1.-8. November 1902. (Die oberste Kurve ist stets die von $0 \mathrm{~m}$, die unterste die ron $6 \mathrm{~m}$ Tiefe.)

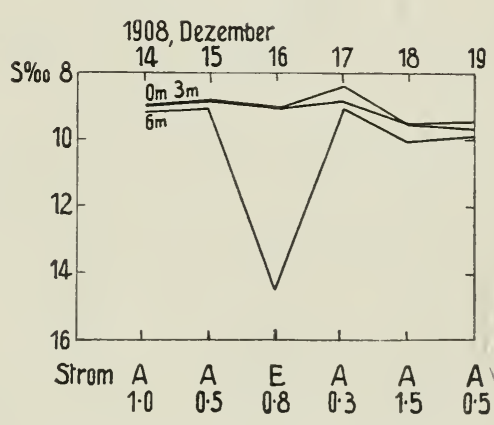

Fig. 7. Salzgehalt und Strom in $0 \mathrm{~m}$, $3 \mathrm{~m}$ und $6 \mathrm{~m}$ Tiefe bei Feuerschiff Drogden rom 14.-19. Dezember 1908. (Die oberste Kurve ist stets die von $0 \mathrm{~m}$.)

Das Gleiche kann sich mit der Zunahme vollziehen, wie der 12.-13. November 1906 bekundeten, denen zwei Tage später sogar erst das Maximum am Boden folgte, nachdem sich an der Oberfläche rorübergehend Auslauf eingestellt hatte. 


\begin{tabular}{l|l|l|l}
\hline Novir. 1906 & $11 \mathrm{~m}$ & $3 \mathrm{~m}$ & $6 \mathrm{~m}$ \\
\hline 11. & $2.2,8$ & 22,8 & 22,8 \\
1.2. & 21,9 & 24,9 & 25,1 \\
13. & 24.8 & 25,1 & 25,1 \\
14. & 12,8 & 17,1 & 24,6 \\
15. & 19,4 & 22,5 & 25,4 \\
\hline
\end{tabular}

Fragen wir nach dem Grunde der Salzgehaltszunahme, so ist klar, dab sie nicht auf einer Durchmischung beruhen kann. Wenn eine solche hier bei Stürmen zweifellos eintritt, wie uns die anderen Feuerschiffe noch dartun werden, so ist die Differenz zwischen Oberfläche und Boden doch so gering, daß ein Produkt beider nicht eine so starke Vermehrung des Salzgehaltes hervorzurufen imstande wäre. Sie kann lediglich durch die Zufuhr von Einlaufwasser aus dem Norden verursacht sein, wie auch solches nicht nur bei Stürmen, sondern gelegentlich auch bei ruhigem Wetter erfolgt, so vom 14.-18. Dezbr. 1908. Die Zunahme des Salzgehaltes spielt sich nur in $6 \mathrm{~m}$ Tiefe ab, selbst in $3 \mathrm{~m}$ Tiefe ist noch nichts von ihr zu bemerken (Fig. 7). Der Sprung kann nur durch Einlaufwass 2 r herbeigeführt worden sein, und in der Tat zeigt ein Vergleich zwischen Stromrichtung und Zunahme des Salzgehaltes fast immer eine Konformität der Erscheinungen, indem einlaufender Strom salzreicheres Wasser aus dem Norden heranfrachtet und mit auslaufendem Strom wieder salzärmeres aus der Arkonasee an seine Stelle tritt. In der Regel pflegt bei der Abwicklung dieses Prozesses die ganze Wassersäule eine einheitliche Stromrichtung anzunehmen, und auch das soeben angeführte Diagramm scheint auf einlaufenden Strom in der ganzen Wasserschicht hinzudeuten. Zieht man jedoch die gesamten vierstündigen Strombeobachtungen in Betracht, wie sie uns die folgend $\rightarrow$ Tabelle für diese Zeit ruhiger Witterung zeigt,

\begin{tabular}{|c|c|c|c|c|c|c|}
\hline Dezbr. 1908 & $4 \mathrm{a}$ & Sa & Mittag & $4 p$ & $8 p$ & Mitternacht \\
\hline 14. & A 1,0 & A 1,0 & A 0,3 & A 0.5 & A 0,3 & A 0,3 \\
\hline 15. & A 0,3 & A $0, \tilde{\delta}$ & A 1,0 & A 0,3 & A 0,3 & C \\
\hline 16. & C & $\mathrm{E} 0,8$ & $\mathrm{C}$ & $\mathrm{C}$ & C & C \\
\hline 17. & C & A 0,3 & A 0,3 & A 1,0 & A 1,0 & A 1,3 \\
\hline 18. & A $1, \overline{0}$ & A $1, \check{5}$ & A 1,2 & A 1,3 & A 1,0 & A 1,1$)$ \\
\hline
\end{tabular}

so erkennt man, daß der Strom bei dem längeren Stillstand um den 16. Dezember gerade während der Salzgehaltsbestimmung um 8 Uhr morgens am 16. ein wenig einwärts setzte; vor und nach 8 Uhr scheint er bei leichtem südlichen Wind in der Tiefe dieses auch schon getan zu haben, während er oberflächlich zum Stillstand gekommen ist. Der 
derart entwickelte Sprung kann sich in viel größerem Maßstabe ausbilden; so waren am 25. November 1902 in $6 \mathrm{~m}$ Tiefe 20,4\% Salzgehalt gemessen, in $3 \mathrm{~m}$ Tiefe aber nur $10,2 \%$ und in $0 \mathrm{~m} 9,5 \%$. Diese Phase, die an und für sich selten ist, stellt sich nur bei ruhigem Wetter ein.

Daß man bei Drogden auf Grund des Salzgehalts zwischen oberflächlich ausgehendem Strom und gleichzeitig am Untergrunde einlaufendem Strom unterscheiden kann, kommt bei der geringen Mächtigkeit des Wassers außerordentlich selten vor. Nachstehende Zahlen geben eins der wenigen Beispiele, bei frischem Winde, der nur am Mittag des 1. November 1910 einmal Sturmstärke 8 erreicht.

Strom:

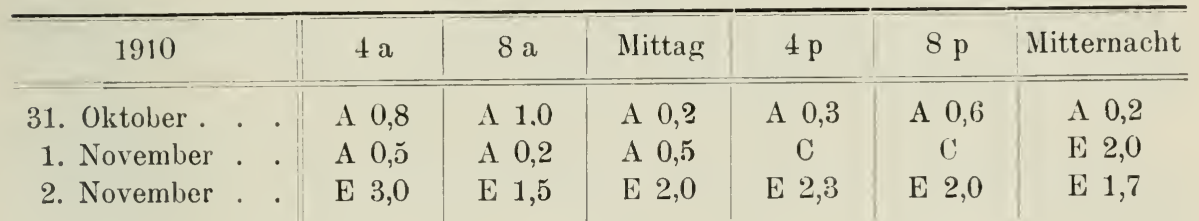

Salzgehalt:

\begin{tabular}{c||r|r|r}
\hline 1910 & $0 \mathrm{~m}$ & $5 \mathrm{~m}$ & $8 \mathrm{~m}$ \\
\hline \hline 31. Oktober . . . & 7,5 & 7,5 & 8,0 \\
1. November . . & 7,8 & 7,8 & 21,3 \\
2. November . . & 22,8 & 22,8 & 22,8
\end{tabular}

Bei oberflächlich auslaufendem Strom ist am 1. November 1910 8 Uhr morgens in $8 \mathrm{~m}$ Tiefe ein Salzgehalt von $21,3 \%$, eine Zunahme von $13,3 \%$ in 24 Stunden. Sie kann nur durch eingehenden Strom veranlaßt sein, der dann auch nach acht Stunden schon den ausgehenden Oberflächenstrom zum Stehen gebracht hat und nach sechzehn Stunden zur alleinigen Herrschaft gelangt ist. In der Regel geht dagegen eine Zunahme in der Tiefe bei fast noch unverändertem Salzgehalt an der Oberfläche und hier gleichzeitig ausgehendem Strom auf einen kurz dauernden Einlauf zurück, der die ganze Wassersäule beherrscht und der nicht zum Ausdruck käme, wenn nur 24stündige Strombeobachtungen vorlägen. Wir geben als solches Beispiel die Zahlen vom 8.-10. November 1908.

Strom:

\begin{tabular}{|c|c|c|c|c|c|c|}
\hline 1908 & $4 \mathrm{a}$ & $8 \mathrm{a}$ & Mittag & $4 p$ & $8 p$ & Mitternacht \\
\hline 8. November & A 1,0 & A 1,0 & A 0,8 & A 1,0 & E $1, \bar{j}$ & E 1,8 \\
\hline 9. November & A 1,3 & A 1,5 & A 1,0 & A 1,0 & C & E 1,0 \\
\hline 10. November & E 1,5 & E 1,5 & E 1,8 & E 1,8 & E $1, \check{\partial}$ & E 1,3 \\
\hline
\end{tabular}

Spethmann, Ozeanographie. Iydrogr. Suppl. z. V.Bd. 
Sialzgehalt:

\begin{tabular}{|c|c|c|c|c|}
\hline 1910 & & () $\mathrm{m}$ & $3 \mathrm{~m}$ & $6 \mathrm{~m}$ \\
\hline 8 November & . & 9,9 & 13,8 & $16 ; 7$ \\
\hline 9. November & . & 8,9 & 12,3 & 18,7 \\
\hline 10. Norember & . & 22,0 & $2 \cdot 2,1$ & $2 \cdot 2,1$ \\
\hline
\end{tabular}

Das Anwachsen des Salzgehaltes in $6 \mathrm{~m}$ Tiefe vom 8.- 9. November ist sicherlich eine Folge des bereits seit dem 8. November abends 8 Uhr einlaufenden Stromes. Ob er am ganzen nächsten Tage in der Tiefe noch vorhanden war und über ihm auslaufender Strom floB, oder ob der am Morgen des 9. November gemessene Salzgehalt von $18,7^{\circ}{ }_{00}$ schon wieder ein Abklingen eines überschrittenen Maximums war, läßt s:ch im vorliegenden Falle nicht definitiv entscheiden.

In einer Reihe anderer Vorkommnisse trifft das letztere aber zu. So ist bei ständig auslaufendem Oberflächenstrom, der eine Geschwindigkeit bis zu 2,5 $\mathrm{Sm}$. besaB, am 12. Juni 1909 doch der Salzgehalt in der Tiefe von $8 \mathrm{~m}$ beträchtlich gewachsen.

Salzgehalt:

\begin{tabular}{l|l|l|l}
\hline 1909 & $0 \mathrm{~m}$ & $5 \mathrm{~m}$ & $8 \mathrm{~m}$ \\
\hline \hline 11. VI. & 8,1 & 12,1 & 14,2 \\
12. VI. & 9,7 & 13,7 & 15,0 \\
13. VI. & 8,1 & 10,1 & 11,1
\end{tabular}

Dieser Zustand ist nicht anders zu erklären, als daß von Norden salzreicheres Wasser unter dem ausfließenden eindrang: er ist selten, im allgemeinen ist bei Drogden die Stromrichtung an der Oberfläche ein sehr feiner Wertmesser für den gesamten Salzgehalt. Die geringe Tiefe der Schwellen scheidet eben das Wasser des Kattegat scharf ron dem der Arkonasee.

Lappe Grund zeigt im Salzgehalt naturgemäß viel Verwandtschaft mit Drogden. Bei ruhigerem, längere Zeit dauerndem Auslauf ist bei ihm die Oberfläche mit einer Decke Auslaufwassers überzogen, die einen Salzgehalt ron $7,5 \%$, wie am 9. Dezember 1903, ausnahmsweise annehmen kann und die umso dünner wird, ie stationärer der Zustand ist. Sie erreicht alsdann keine 5-10 m, schon in $5 \mathrm{~m}$ Tiefe findet sich um 8-10\% salzreicheres Wasser; der Sprung kann hier sogar den hohen Betrag von $18,6 \%$ erreichen, wie es am 23. März 1912 vorgeliommen ist. In $15 \mathrm{~m}$ und mehr Tiefe stellt sich bei ruhigem Auslauf eine konstante Anreicherung von Wasser ein, dessen Salzgehalt zwischen 28 und $34^{\circ}$ oo 
gelegen ist. Er schwankt in den einzelnen Auslaufperioden in 20 und 23 m nur wenig, so keine $4 \%$ vo vom 26. Februar 1908 bis 28. März 1908. In 15 m Tiefe macht der Salzgehalt dagegen des öfteren eine sofort in die Augen fallende Wellung, die dem Salzgehalt der Tiefen von 5 und $10 \mathrm{~m}$ eigen ist. Sie bildet die Sprungschicht, die bald ein höheres, bald ein tieferes Niveau einnimmt, wie die auf- und abgehenden Kurven bekunden. Verfolgt man ihre Maxima und Minima, so kann man ab und zu gewisse Regalmäßigkeiten in Gestalt zwei- und dreitägiger Perioden erkennen, zumal wenn man die Kurven für $5 \mathrm{~m}$, $10 \mathrm{~m}$ und $15 \mathrm{~m}$ Tiefe gleichzeitig um eine gewisse Mittellage des Salzgehaltes verfolgt, aber es scheint, daß sich aus den 24 stündigen Beobachtungen allein keine Schlüsse über die Art und Ursache der Schwanliungen ableiten läßt, so daß wir es offen lassen müssen, ob seichesartige Bewegungen oder Gezeitenwogen vorliegen. Wir werden sie später bei Fehmarnbelt wiedersehen.

Diese Verhältnisse ruhigen Auslaufes werden durch Stürme, die den Auslauf verstärken, noch schärfer herausgearbeitet, wenn sich auch vorübergehend eine Störung infolge Durchmischens einstellt. So bekunden der 1. bis 8. November 1902, wie ein ESE-Sturm eine Durchmischung bringt (Fig. 8). Am besten lassen sich die Veränderungen überblicken, wenn auf eine längere Zeit auslaufenden Stromes bei südlichen und östlichen Winden ein Sturm aus etwa SE ankommt. Finen solchen Fall bietet die Zeit nach dem 8. Februar 1904, die als wesentlichen Einfluß das Wachsen der Mächtigkeit der Süßwasserdecke zeigt.

Umgekehrt macht sich auf Lappe Grund jeder Sturm mit Einlauf an der Oberfläche mit einer Anreicherung des Salzgehaltes bemerkbar. Hand in Hand mit ihr geht eine Abnahme der Zunahme in die Tiefe, so daß eine Durchmischung eintritt, die um so intensiver und andauernder ist, je stärker und länger der Sturm wird. Der 6.-13. Januar 1903 und der 18. und 30. Januar 1903 zeigen verschiedene Stadien einer nicht gänzlich erfolgten Durchmischung, am 7. April $190 \pm$ sehen wir eine, in der das Wasser von der Oberfläche bis $23 \mathrm{~m}$ Tiefe nur um $0,5 \%$ schwankt. Gleich beim Nachlassen des Sturmes setzt auch schon die Schichtung wieder ein, so daß bei mehrfachem Einlauf und Auslauf sich ebenso häufig ein Durchmischen und eine Schichtung vollzieht, wie der 2. und 4. November 1903 wiedergeben (vgl. Fig. 9). Die Neubildung der Schichtung pflegt schneller nach der Tiefe hin als nach der Oberfläche zu erfolgen, wo der nachlassende Wind im Verein mit dem nicht so schnell verlaufenden Seegang noch vermengend auf die 
Wasserteilchen wirkt. Yom 25.-28. November 1903 oder 6.-13. April 1904 ist dieses deutlich wahrnehmbar.

Neben der derart in der Regel sich vollziehenden Beeinflussung durch Einlaufstürme wollen wir eine bemerkenswerte Ausnahme bstrachten. Ein WNW brachte am 23. Juni 1904 eine Durchmischung, die bei einlaufendem Strom sich nicht in einer Abnahıne des Salz-

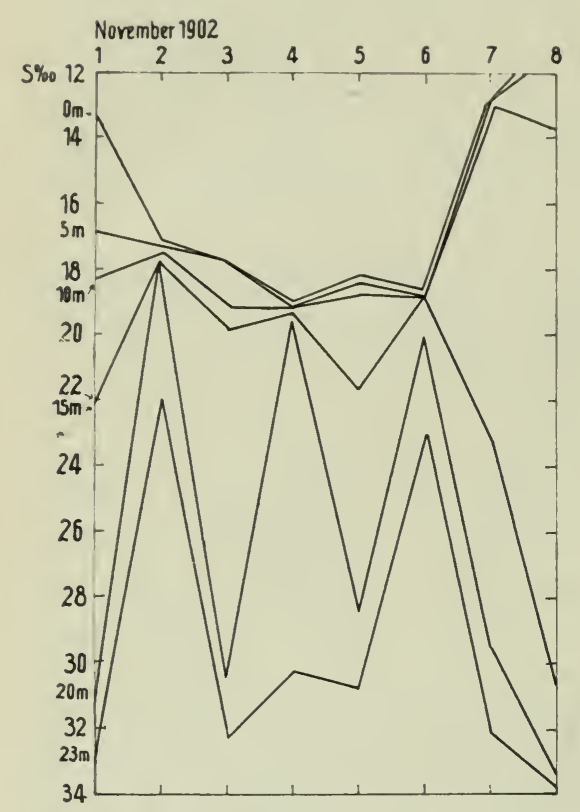

Fig. 8. Salzgehalt in $0 \mathrm{~m}, \overline{\mathrm{j}}, 10 \mathrm{~m}$, $15 \mathrm{~m}, 20 \mathrm{~m}$ und $23 \mathrm{~m}$ Tiefe bei Feuerschiff Lappe Grund rom 1.-8. Nov. 1902.

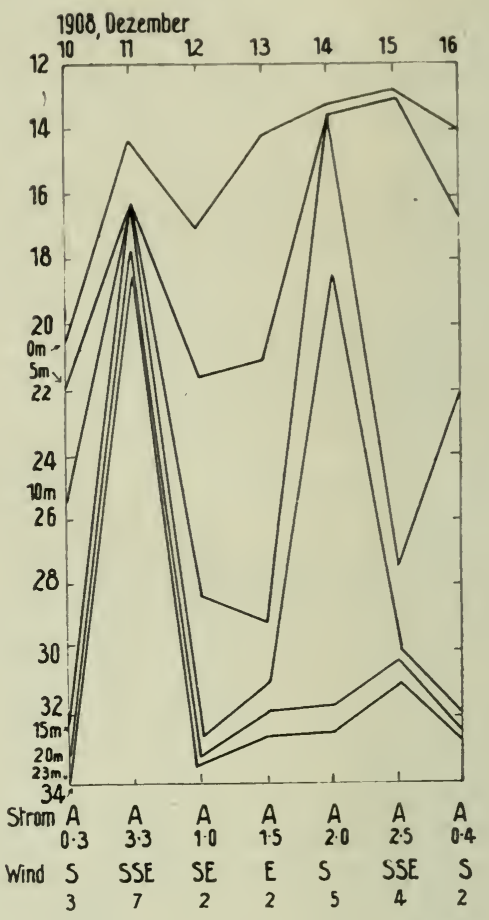

Fig. 9. Salzgehalt, Strom und Wind in $0 \mathrm{~m}, 5 \mathrm{~m}, 10 \mathrm{~m}, 15 \mathrm{~m}, 20 \mathrm{~m}$ und $23 \mathrm{~m}$ Tiefe beim Feuerschiff Lappe Grund vom 10. bis 16. Dezember 1908.

gehaltes bis zu $15 \mathrm{~m}$ Tiefe erkennen lieB. Die Ursache beruht darauf, daß schon seit dem 17. Juni Einlauf herrschte, der bereits am 20. Juni den Salzgehalt der Oberfläche auf $19 \%$ gehoben hatte. Bei einer solchen Sachlage geschieht eine Durchmischung ohne erhebliche Änderung des Salzgehaltes in den oberen Schichten (Fig. 10).

Die Einlauf- und Auslaufwirkungen auf Schultz Grund lassen sich nicht besonders klar überblicken, was mit örtlichen Abweichungen des generellen Verlaufes zusammenzuhängen scheint. Betrachten wir zu- 
nächst die kräftige Einwirkung der Stürme. Sie rufen viel səltener als bei den beiden behandelten Feuerschiffen einə Durchmischung hervor, wie am 4. Januar 1905, wo infolge eines stark einlaufenden Stromes, den Sturm aus westlichen Richtungen brachte, die Wassersäule von der Oberfläche bis zum Boden nur zwischen 26,0 und 26,1\% differierte. Im allgemeinen beträgt auch bei heftigen Luftbewegungen der Unterschied mehrere Promille sowohl bei Einlauf als auch bei Auslauf. Ja, Stürme spielen sich öfters ab, ohne die tieferen Schichten irgendwie zu beein-

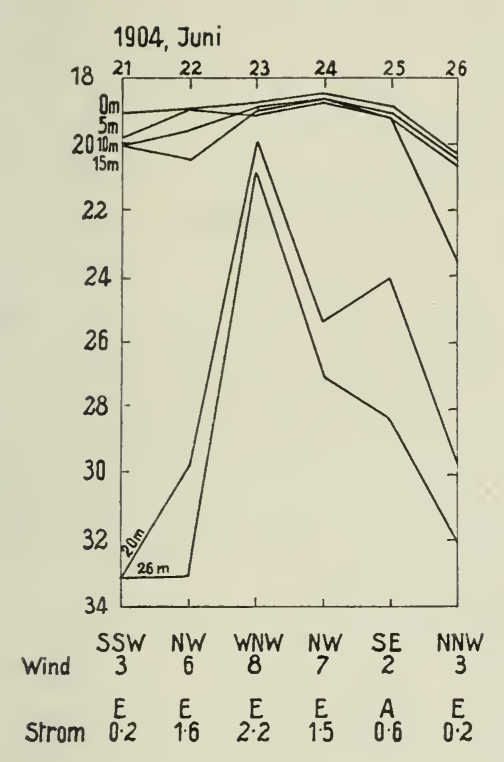

Fig. 10. Salzgehalt, Wind und Strom in $0 \mathrm{~m}, 5 \mathrm{~m}, 10 \mathrm{~m}, 15 \mathrm{~m}, 20 \mathrm{~m}$ und $26 \mathrm{~m}$ Tiefe beim Feuerschiff Lappe Grund rom 21.-26. Juni 1904.

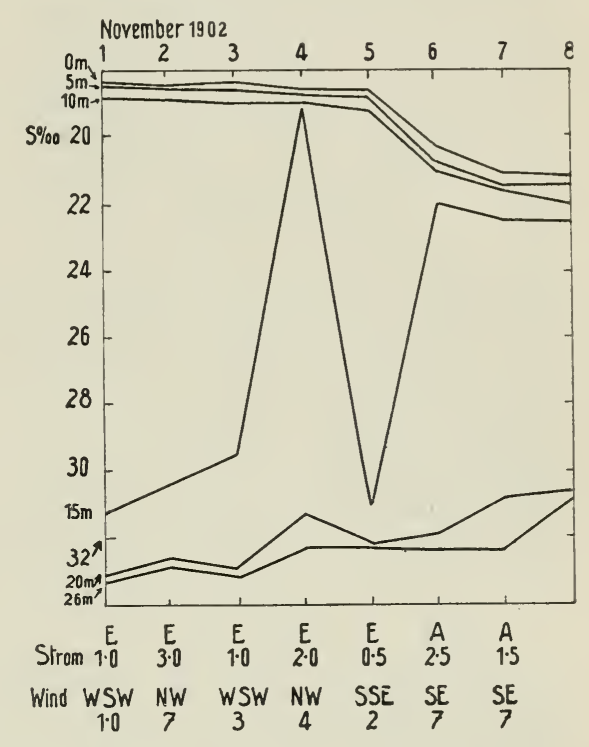

Fig. 11. Salzgehalt, Strom und Wind in $0 \mathrm{~m}, 5 \mathrm{~m}, 10 \mathrm{~m}, 15 \mathrm{~m}, 20 \mathrm{~m}$ und $26 \mathrm{~m}$ Tiefe beim Feuerschiff Schultz Grund rom 1.-8. November 1902.

flussen. Der Einlauf bringt vielfach, aber keineswegs immer, ein Anschwellen der Mächtigkeit des salzreichen Bodenwassers mit starker Sprungschicht darüber. Mitunter äußert sich aber der Einlauf nicht in einem solchen Anschwellen, sondern nur in einem Wachsen des Salzgehaltes, wie am 21. Juni 1904 in $20 \mathrm{~m}$ von 32,9 auf 33,2 und in $26 \mathrm{~m}$ von 33,2 auf 33,4 .

Starker Auslauf bringt begreiflicherweise süßeres Wasser an der Oberfläche, das scharf gegen salzreiches Wasser in der Tiefe absetzt, dessen Salzgehalt oft noch während des Sturmes gesteigert wird, augenscheinlich die Wirkung einer Reaktionsströmung, wie Anfang November 
1906. Der ansliufende Strom bietet keineswegs immer bei Sturm die niedrigsten Salzgehaltswerte, denen infolge der Durchmischung der obersten Schichten doch immer wieder salzreiches Wasser zugeführt wird. Hierbei kamn der Auslauf an der Oberfläche sogar eine Zunahne des Salzgehaltes erfahren, wie Fig. 11 zeigt, wo der Sturm am 6. morgens 4 Uhr losbrach. Dementsprechend treten die niedrigsten Salzwerte nicht bei Stürmen mit Durchmischung auf, sondern bei ruhigem, stetigem Auslauf. Bei diesem ereignete sich auch am 26. September 1909 der abnorm niedrige Salzgehalt von $9,7^{\circ} / 00$ an der Oberfläche und $11,0^{\prime \prime} / 00$ in $5 \mathrm{~m}$ Tiefe! Erst in $10 \mathrm{~m}$ Tiefe maß man $16,7 \%$ ! Andererseits treten bei ruhigem Auslauf auch Salzgehaltswerte ein, die keinesfalls besonders niedrig sind. So ereigneten sich beispielsweise vom 1.-10. Februar 1904 Schwankungen zwischen $18,9^{\circ} \%$ und $22,1 \%$ an der Oberfläche, trotzdem der Strom, wenn auch nicht sehr kräftigr, so doch ständig auslief. Er war dafür bis zu $15 \mathrm{~m}$ mächtig, wo das Wasser mit einem scharfen Sprung gegen solches von über 30,8\% absetzte. Auch der umgekehrte Fall kann eintreten, daß Einlauf auffallend salzarmes Wasser bringt. Es kommt aber mit Strom aus SE, der das nahe der Nordküste Fünens befindliche und vom Lande herrührende süße Wasser heranfrachtet, so am 29. September an'der Obesfläche einen Salzgehalt von $12,9^{0}{ }_{00}$. Er kann gemäß seiner Herkunft nicht sehr mächtig gewesen sein, denn $5 \mathrm{~m}$ tief waren bereits $19,4^{\prime \prime} \%_{00}$.

Nach der Diskussion der Salzgehaltsbewegungen auf drei Feuerschiffen können wir uns bei Gjedser Rev kurz fassen. Auslauf und Einlauf vollziehen sich bei ihm während ruhigen Wetters in der ganzen Mächtigkeit von $11 \mathrm{~m}$, wobei gegenüber Drogden infolge der größeren Tiefe eine leichte Zunahme des Salzgehaltes nach dem Boden in der Regel deutlich wahrzunehmen ist. Das Wechseln der Stromrichtung erfolgt oft schon an der Oberfläche, während es in der Tiefe noch nicht eingetreten ist, aber mit den verschiedenen Phasen der Verzögerung (14. bis 20. August $1^{\complement} 09$ ), die wir bei Drogden kennen lernten: auch gehören hier wie dort verschiedene Stromrichtungen übereinander zu den Ausnahmen, ereignen sich aber für die Dauer eines Tages doch öfters, wofür der 16. bis 18. Oktober 1905 als Beispiel angeführt sein mögen.

Salzgehalt:

\begin{tabular}{c|l|l|l}
\hline 1905 & $0 \mathrm{~m}$ & $5 \mathrm{~m}$ & $11 \mathrm{~m}$ \\
\hline $16 . \mathrm{X}$. & $\mathbf{1 3 , 0}$ & $\mathbf{1 3 , 0}$ & 13,1 \\
$17 . \mathrm{X}$. & 12,9 & $\mathbf{1 3 , 0}$ & $\mathbf{1 4 , 0}$ \\
$18 . \mathrm{X}$. & 11,9 & 12,2 & 12,4
\end{tabular}


Bei Stürmen sind diese Phasen natürlich schärfer ausgearbeitet, Figur 13 zeigt, wie erst nach vier Tagen die Unterschiede im Salzgehalt sich mindern. Einlauf bringt natürlich salzreicheres, Auslauf salzärmeres Wasser herbei (Figur 12). Stromstillen, die ein Charakteristikum für Gjedser Rev abgeben, ereignen sich meistens bei hohem Salzgehalt, da Einlauf den Auslauf zurückstaut. Als guten Beleg bringen wir die Messungen vom 11. bis 15. Februar 1903.

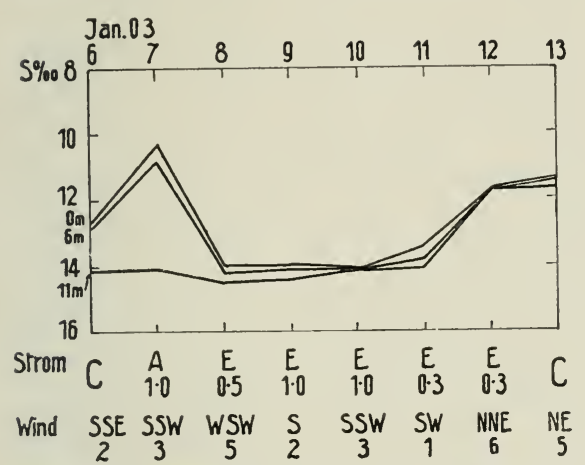

Fig. 12. Salzgehalt, Strom und Wind in $0 \mathrm{~m}, 5 \mathrm{~m}$ und $11 \mathrm{~m}$ Tiefe beim Feuerschiff Gjedser Rev vom 6.-13. Januar 1903. (Die höchste Kurve ist stets die von $0 \mathrm{~m}$, die tiefste stets die ron $11 \mathrm{~m}$ Tiefe.)

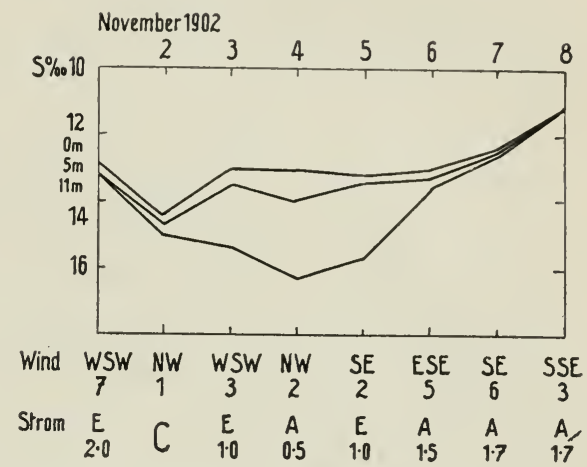

Fig. 13. Salzgehalt, Wind und Strom in $0 \mathrm{~m}, 5 \mathrm{~m}$ und $10 \mathrm{~m}$ Tiefe beim Feuerschiff Gjedser Rer rom 1.-8. Nov. 1902. (Die unterste Kurve ist stets die von $11 \mathrm{~m}$ Tiefe.)

Strom:

\begin{tabular}{c|c|c|c|c|c|c}
\hline 1903 & $4 \mathrm{a}$ & $8 \mathrm{a}$ & Mittag & $4 \mathrm{p}$ & $8 \mathrm{p}$ & Mitternacht \\
\hline \hline 11. II. & $\mathrm{E} 0,5$ & $\mathrm{C}$ & $\mathrm{C}$ & $\mathrm{A} 0,5$ & $\mathrm{C}$ & $\mathrm{C}$ \\
12. II. & $\mathrm{C}$ & $\mathrm{E} 2,0$ & $\mathrm{~A} 1,5$ & $\mathrm{C}$ & $\mathrm{C}$ & $\mathrm{C}$ \\
13. II. & $\mathrm{C}$ & $\mathrm{C}$ & $\mathrm{C}$ & $\mathrm{E} 1,5$ & $\mathrm{E} 2,0$ & $\mathrm{E} 1,0$ \\
14. II. & $\mathrm{C}$ & $\mathrm{C}$ & $\mathrm{A} 0,5$ & $\mathrm{~A} 0,7$ & $\mathrm{~A} 0,5$ & $\mathrm{~A} 1,0$ \\
15. II. & $\mathrm{A} 1,0$ & $\mathrm{~A} 0,5$ & $\mathrm{~A} 0,5$ & $\mathrm{C}$ & $\mathrm{C}$ & $\mathrm{A} 0,5$
\end{tabular}

Salzgehalt:

\begin{tabular}{c|c|c|c}
\hline 1903 & $0 \mathrm{~m}$ & $5 \mathrm{~m}$ & $11 \mathrm{~m}$ \\
\hline 11. II. & 16,4 & 16,4 & 16,4 \\
12. II. & 16,8 & 16,9 & 17,1 \\
13. II. & 18,1 & 18,3 & 18,4 \\
14. II. & 18,6 & 18,6 & 18,6 \\
15. II. & 16,3 & 17,3 & 18,0
\end{tabular}

Es kann aber auch bei Stromstille eine Abnahme eintreten, wie der 9.-11. September 1907 gelehrt haben. Ihre Ursache liegt nicht etwa in einer Differenzierung nach der Tiefe zu, so dab es scheint, als ob zwischen 
den Beobachtungszeiten doch leichte Strömungen geherrscht haben, wie es sich denn überhaupt nur um geringe Salygehaltsunterschiede handelt.

Eho wir an die Zusammenfassung der Ergebnisse herangehen, wollen wir noch Beobachtungen vom Feuerschiff Fehmarnbelt einschalten. Hier werden zwar auch regelmäßige Messungen wie an den dänischen Feuerschiffen ausgeführt, aber sie stehen nicht unter so scharfer Kontrolle und sind nicht so umfassend, so daß wir sio bei der Diskussion der Ergebnisse an den dänischen Schiffen einstweilen ausgeschaltet haben, um erst bei Besprechung der internationalen Terminfahrten auf sie zurückzukommen. Dagegen hat C. Carp vom 13. August bis 10. September 1909 auf Fehmarnbelt Salzgehaltsmessungen für verschiedene Tiefen halbtägig vorgenommen. Die Proben waren mit l'etterssons Wasserschöpfer genommen und sind im Berliner Institut für Meereskunde titriert worden, so daß wir die Resultate als kleine willkommene und zuverlässige Ergänzung mit den übrigen Feuerschiffen in Vergleich stellen können. Sie liegen glücklicherweise so, daß sie die Wiıkungen zweier Stürme und einer ruhigen Periode deutlich erkeinnen lassen. Bei der letzteren entwiclielte sich bei auslaufendem Strom zwischen 10 und $20 \mathrm{~m}$ Tiefe eine Sprungschicht mit einer durchschnittlichen Salzgehaltsdifferenz von etwa $4 \%$. Unter ihr war in der Regel Einlauf. Der eine Sturm rom 5. bis 6. September, der Einlauf brachte, zerstörte sie gänzlich und verursachte eine Durchmischung, so daß die Differenzierung des Salzgehaltes bis $26 \mathrm{~m}$ Tiefe nur $2 \%$ ausmachte und Einlauf in der ganzen Wassersäule herrschte. Der andere Sturm, gleichfalls mit Einlauf, machte sich nur bis 20 m Tiefe bemerkbar, der Salzgehalt am Boden wurde nicht mehr beeinflußt. Für die Stromrichtung in der Tiefe fehlen bei diesem Sturm die Beobachtungen.

Dio Salzgehaltsbewegungen zeigen, im ganzen betrachtet, an der Oberfläche den gleichen Gang, ein ausgeprägtes Minimum im Mai, das nur bei Schultz Grund einen Monat später fällt, ein ausgesprochenes Maximum im Januar, das gleichfalls bei Schultz Grund einen Monat nachhinkt. Zwei sekundäre Minima fallen auf März und September, ron denen das eine bei Schultz Grund sich ebenfalls um einen Monat verspätet, während das andere nicht entwickelt ist. Ferner bringt der Nordausgang des Sundes noch im Dezember ein sekundäres Minimum. An sekundären Maximis fällt eins fast bei allen gemeinsam auf Januar, zwei weitere im April und August fehlen bei Schultz Grund (Figur 14).

Die Gemeinsamkeit der Züge der jährlichen' Salzgehaltsbewegungen zeigt sich auch in den Veränderungen von Jahr zu Jahr, wie die Kurven von Fig. 15 dartun. 
Ozeanographie der südwestlichen Ostsee. I.

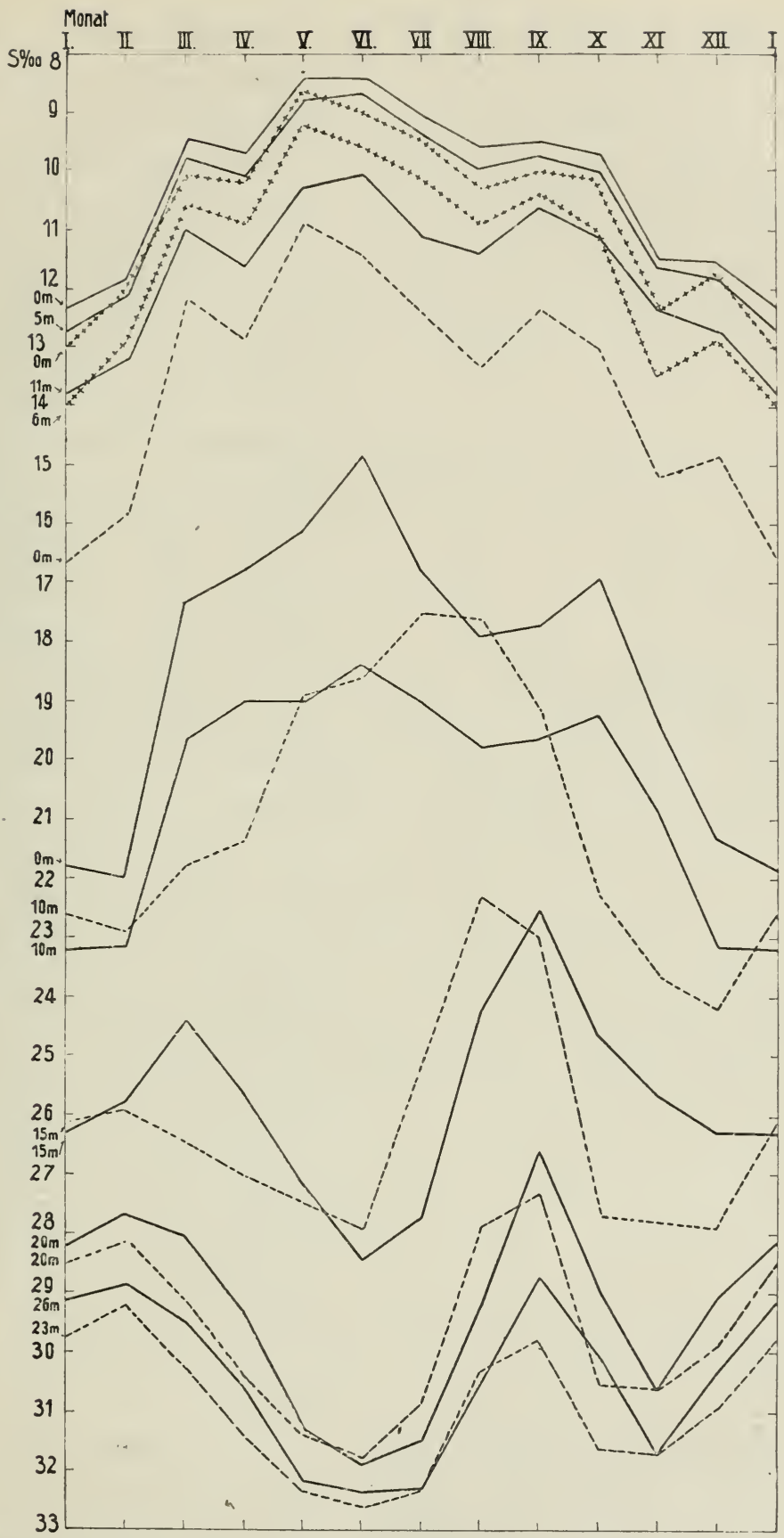

Fig. 14. Monatsmittel des Salzgehaltes bei den Feuerschiffen Drogden, Gjedser Rev, Lappe Grund und Schultz Grund 1902-1911. Kurven für Gjedser Rev und Schultz Grund ausgezogen, für Drogden gekreuzt, für Lappe Grund gestrichelt. 


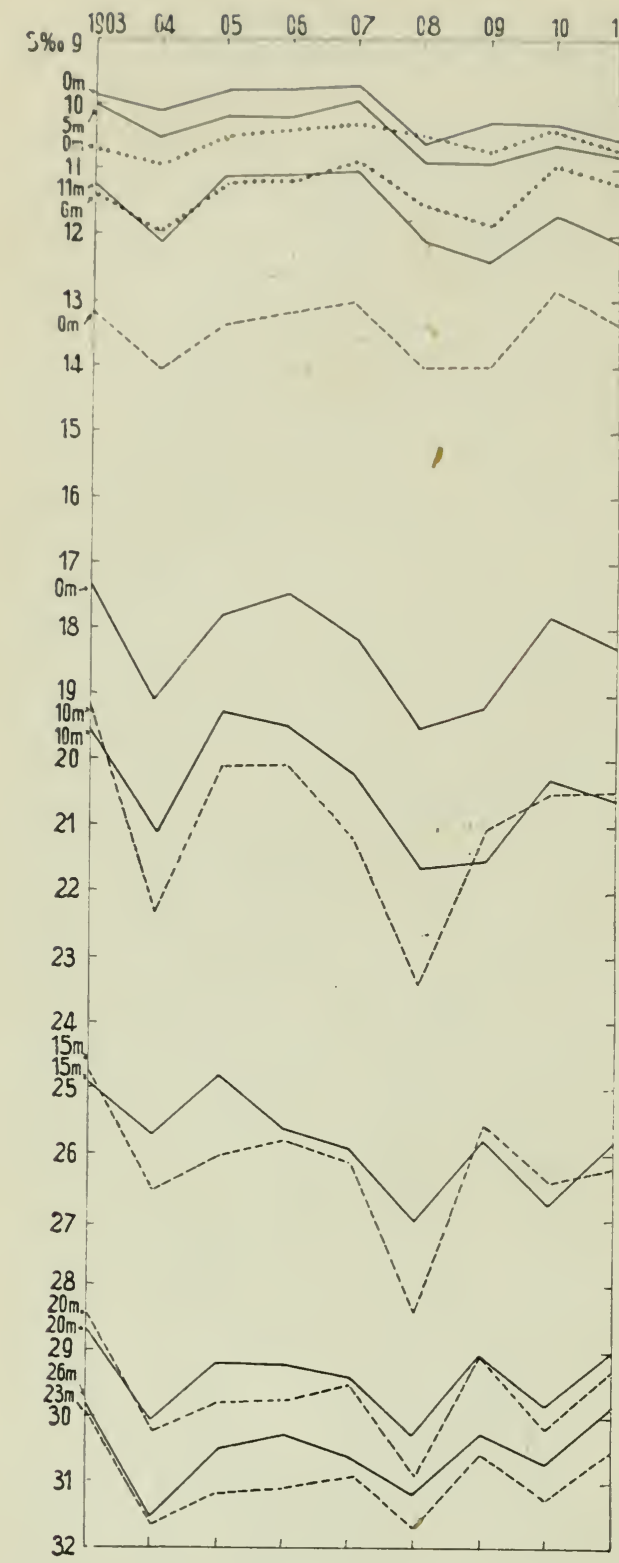

Fig. 15. Jahresmittel des Salzgehaltes bei den Feuerschiffen Drogden, Gjedser Rer, Lappe Grund und Schultz Grund 19031911. Kurven für Gjedser Rev und Schultz Grund ausg̨ezogen. für Drogden gekreuzt, für Lappe Grund gestrichelt.
Deutlich zeigen alle vier Kurven für die (Oberfliiche ein Minimum für das Jahr 190:3, ein Maximum für 190t. Ein anderes Minimum fällt mit Ausnahme bei Schult: Grund auf 1907, ferner besitzt der Sund ein zweites Maximum 1909, der Stammbelt und der GroBe Belt 1908. Auch für die Tiefe gibt sich ein harmonischer Verlauf von "Jahr zu Jahr kund. Lappe G̈rund und Schultz Grund korrespondieren in den großen Maximis, nur ein kleineres von 1906 fehlt bei Schultz Grund. Die Minima verlaufen dagegen nicht so ähnlich, doch sind die Unterschiede nur gering; allgemein liegt ein großes Minimum zwischen zwei Maximis von 1904 und 1908. Das gegenseitige Verhältnis vom Wasser der Oberfläche zur Tiefe ist nicht umgekehrt, hierin bringt das Jahresmittel eine Abweichung rom Monatsmittel. Ist der Salzgehalt eines Jahres an der Oberfläche gering, so pflegt sich auch ein Minimum in der Tiefe einzustellen und umgekehrt. Bei Gjedser Rev besteht für 1908 und 1909 eine kleine Abweichung, während bei Drogden die Tiefe die Bewegungen der Oberfläche wiederholt.

Die mittleren Extreme der Salzgehaltsschwankungen erreichen ihr größtes Ausmaß bei Drogden. Gemäß der geringen Tiefe der Schwelle sind die 
Salzgehalte in den nördlich und südlich anstoßenden Gewässern am kontrastreichsten. Den kleinsten Ausschlag besitzt Schultz Grund, vermag hier doch das Einlaufwasser sich am freiesten auf allen vier Feuerschiffen einzustellen. Die Differenzen der mittleren Extremo zwischen Boden und Oberfläche sind gering bei Drogden, über 1,0"\% gehen sie nicht bei Gjedser Rev, $1,2^{\circ}{ }_{00}$ und 4,0\% erreichen sich bei Schultz Grund und Lappe Grund. Wie za erwarten, sind sie bei den beiden Feuerschiffen größer an der Oberfläche als am Boden, was auch schon für Giedser Rev gilt. Die absoluten Extreme gibt folgende Zusammenstellung:

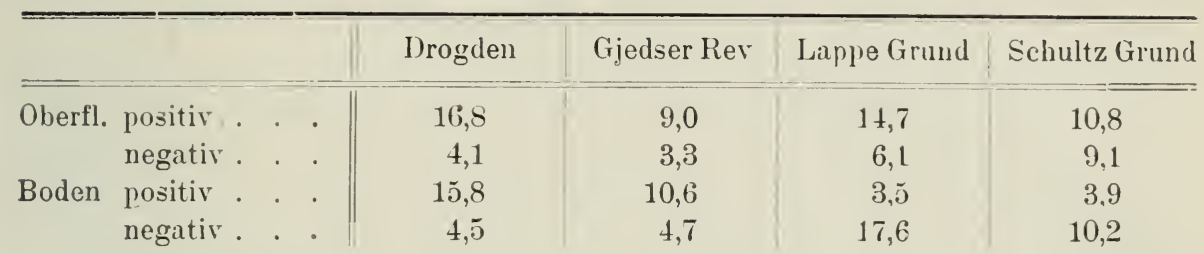

An der Oberfläche liẻzen bei allen vier Feuerschiffen die größten Abweichungen auf der positiven Seite; der Einlauf salzreichen. Wassers ist das anormale. An dem Boden finden wir das gleiche Bild nur bei den beiden südlichen Feuerschiffen, die beiden nördlichen besitzen dagegen die größte Abweichung auf der negativen Seite. Bei diesen kann sich der Salzgehalt nicht viel mehr über die an und für sich schon hohen Nittelwerte von $30,5 \%$ und $31,0 \%$ erheben, $34,5{ }^{\circ}$, 0 ist der höchste, gemessen am Boden ron Lappe Grund am 1. Norember 1906. An der Oberfläche sind dagegen nur 29,1, und zwar auf Schultz Grund, erreicht. Der niedrigste Wert ist natürlich auf Drogden gelegen, $6,5{ }^{\circ}{ }_{00}$ am 1. April 1904, während Gjedser Rev nur bis 6,8\% herabgeht. Aber auch Lappe Grund und Schultz Grund haben für ihre Lage auffallend niedrige Werte aufgewiesen, das erste $7,3{ }^{\circ}$ oo am 29. September 1909 und das letztere am 3. Juni $19119,2{ }^{\circ}{ }_{00}$, ein ganz gewaltig niedriger Salzgehalt bei dem fünfmal längeren Wege von der Arkonasee ins Kattegat als durch den Sund.

Auf die 0 m-Kurven der Monatsmittel kann eine Fülle von Möglichkeiten einwirken; der Wasserstand, die gesamte Süßwasserzufuhr in ihren verschiedenen Phasen, der Wind und das Eis sind die Hauptfaktoren. Trotz mancherlei Abweichungen zeigen die Linien die Grundzüge gemeinsam mit den Kurven der Differenzen der Monatsmittel zwischen Auslauf und Einlauf. Minimum und Maximum decken sich fast ganz bei Gjedser Rev, Drogden und Lappe Grund, bei Schultz Grund treten sie einen Monat später auf. Auch dis sekundären Minima im März und Septem- 
ber sind in der gleichen Weise wieder zu erkennen, ebenso die kleineren Maxima des April, des August und November. Wir sehen also zunächt den starken Einfluß der Verteilung von Einlauf und Auslauf. de stärker der Ausflub, um so geringer der Salygehalt. Diese genetischen Beziehungen bestätigen sich, wenn wir die Veränderungen von Jahr zu Jahr ins Auge fassen. Wir haben die Ergebnisse auf Figur 15 zusammengestellt, deren Vergleich uns die imnige gegenseitige Abhängigkeit zwischen Salzgehalt an der Oberfläche und dem Auslauf lehrt. Maxima und Minima decken sich fast völlig in ihren großen Zügen, nur in der Intensität unterscheiden sie sich. Lediglich die Spitze bei Schultz Grund für die Differenzen von 1908 fällt aus dem Gesamtbild heraus; eine Ursache vermögen wir hierfür nicht anzugeben.

Bei den Monatsmitteln trat nur der starke Auslauf des März nicht so scharf als Minimum in der Salzgehaltskurve in die Erscheinung. Seine Ursache führt uns auf die Wasserführung der Flüsse der südwestlichen Ostsee, deren Menge den Salzgehalt merklich beeinflussen muB. Die gewaltige Abnahme des Salzgehaltes im Frühjahr bis zum Juni findet ihren Vorläufer in den Schwankungen der Wassermengen der näheren größeren Ströme, wie die Pegelstände zeigen. Auf Grund längerer Reihen ergeben sich nach den Stromwerken folgende Werte für den Unterlauf von Oder und Weichsel.

Pegelstand in $\mathrm{cm}$.

\begin{tabular}{l||l|l|l|l|l|l|l|l|l|l|l|l|l|l|}
\hline & Jan. & Feb. & Mărz & April & Mai & Juni & Juli & Aug. & Sept. & Olit. & Nov. & Dez. \\
\hline Stettin . & 0,61 & 0,66 & 0,75 & 0,76 & 0,59 & 0,63 & 0,66 & 0,67 & 0,61 & 0,58 & 0,58 & 0,63 \\
Marienburg - & 2,32 & 2,49 & 2,95 & 2,92 & 2,00 & 1,54 & 1,42 & $1,3 \check{5}$ & 1,14 & 1,14 & 1,39 & 1,91
\end{tabular}

In der Tiefe zeigen die Salzgehaltsschwankungen bei Drogden der geringen Niveaudifferenz halber die kleinsten Abweichungen von der Oberfläche. Auch $5 \mathrm{~m}$ und $11 \mathrm{~m}$ bei Gjedser Rev wiederholen das Bild der Oberfläche, nur daß in $11 \mathrm{~m}$ Tiefe das sommerliche Minimum, das sich bei $0 \mathrm{~m}$ und $5 \mathrm{~m}$ auf 2 Monate ausbreitet, auf einen, den Juni, beschränkt und wir somit die einmonatliche Verschiebung, die Schultz Grund nach dieser Richtung erfährt, hier auch angedeutet finden. Auch bei Schultz Grund zeigen sich in $10 \mathrm{~m}$ Tiefe noch keine durchgehenden Abweichungen von der Oberfläche, nur daß das JuniMinimum nicht so scharf herausgearbeitet ist; bei Lappe Grund ergeben sich dagegen schon recht erhebliche Abweichungen. Die Kurve zeigt nur ein Minimum von Juni bis August, ein absolutes Maximum 
im Dezember und ein sekundäres Maximum im Februar. Der Verlauf dieser Kurven wird uns erst verständlich, wenn wir die Salzgehaltsbewegungen des tieferen Wassers betrachten. Am klarsten ist der Kontrast bei Schultz Grund zwischen $10-15 \mathrm{~m}$. Bei $15 \mathrm{~m}$ entspricht dem Juni-Minimum des höheren Wassers ein Juni-Maximum, die Kurve verläuft vom März bis August entgegengesetzt, dagegen nicht während des übrigen Teiles des Jahres, in welchem sie um einen Monat den Bewegungen des Salzgehalts in den höheren Wasserschichten vorauseilt. Ein ähnliches Bild bietet Lappe Grund. Auch auf ihm wird die Kurve nach der Tiefe hin einfacher, was in beiden Fällen seine Ursache in dem Ausschalten kleinerer Einwirkungen auf die Oberfläche hat, auch sie verläuft bei $15 \mathrm{~m}$ von Februar bis August entgegen jener der Oberfläche, auch sie geht für den Rest des Jahres der Oberfläche um einen Monat voraus.

Die Kurven bieten uns die Möglichkeit, die Mächtigkeit für Auslauf und Einlauf abzuschätzen. Der Gegensatz in den Salzgehaltsbewegungen zwischen $10-15 \mathrm{~m}$ Tiefe bei Schultz Grund weist auf eine jährliche Mittelgrenze von 12-13 m Tiefe hin, die die Zone der Oberflächenwasserbewegungen von denen der Tiefe trennt. Bei Lappe Grund scheint die Grenze schon bei 11-12 m Tiefe zu liegen, während Drogden und Gjedser Rev zu flach sind, als daß sie zum Ausdruck käme.

Nach Betrachtung der Salzgehaltsschwankungen an den einzelnen Feuerschiffen wollen wir ein synoptisches Bild der vier Stationen zu gewinnen trachten. Deutlich liegen die Einwirkungen ruhigen Auslaufes und ruhigen Einlaufes dar. Sie bringen einen konstanten niedrigen resp. hohen Salzgehalt. Diese klaren Verhältnisse werden durch Stürme gänzlich unterbrochen. Bei Gjedser Rev und Drogden führen sie schnell zu einer gänzlichen Durchmischung, bei Lappe Grund durchdringen sie meistens auch die ganze Wassersäule, nur bei Schultz Grund beschränkt sich der Prozeß in der Regel auf die oberen Schichten. Die Durchmischung erfolgt bei allen Stationen unmittelbar bei Eintritt des Sturmes, so daß sie auch für die dazwischen liegende Wasserfläche zu gelten hat. Wir haben, um diese weitgreifende ozeanographische Tatsache voll zu würdigen, eine Reihe von Stürmen in ihrer gleichzeitigen Wirkung an verschiedenen Stationen für eine Anzahl typischer Fälle ausgearbeitet, nehmen aber von der Wiedergabe der großen Tafeln an dieser Stelle Abstand. Besonders instruktive Beispiele bieten der 18. bis 30. November 1903, der 24. Oktober bis 15. November 1906 und der 13. August bis 10. September 1909. 
Die intensive gleichzeitige Durchmischung des Wassers der Beltsee und des Sundes bei Stürmen lehrt uns das wichtige Resultat, daß das In die Arlionasee einlaufende Wiasser keineswegs lediglich aus salzreichem Wasser an Boden besteht, sondenn sich bei Stürmen mit Einlauf aus einer Nisthung von Auslauf und Einlauf zusammensetzt. kbenso gewinnen wir das Ergebnis, dal der Auslauf bei Stürmen mit Auslauf nicht in einer stärkeren Ausfuhr von Oberflächenwasser der Arkonasee und Gotlandsee besteht, sondem gleichfalls in einer Mischung von Auslauf mit Einlauf. Die Beltsee ist demnach wïhrend einer Sturmperiode eine hydrographisehe Mischregion. Das so erzeugte Wasser gelangt ins Kattegat, wo es die Oberfläche deckt, oder in die Arkonasee, wo es zum Boden wandert. Stoßen wir hier also auf größeren Salzgehalt, so ist es keineswegs immer Wasser des Kattegat, das hierher gelangt ist, indem es bei Anreicherungen vor Schwellen diese gelegentlich stoßweise überschritt, sondern Mischung von Auslauf und Einlauf. Welche der beiden Wasserarten hierbei vorwaltet, ist sehwer abzuschätzen. Zwar überwiegen die Tage mit geringen Windgeschwindigkeiten zweifellos jene mit Stürmen, doch brauchen die Wasserpartikel zum Zurücklegen des Weges durch Beltsee und Sund eine gewisse Zeit. Legen wir beispielsweise für die kürzeste Strecke durch den Sund eine mittlere Stromgeschwindigkeit von 0,8 Knoten in der Stunde zugrunde, so erhalten wir für den rund etwa $30 \mathrm{Sm}$ langen Weg eine Wanderdauer von $1^{1} / 2$ Tag, während wir von Schultz Grund bis zur Darsser Schwelle die fünffache Zeit rechnen müssen. Hierzu kommt, daß die Wasserpartiliel nicht den kürzesten Weg zurücklegen, sondern neben der Rechtsdrängung infolge der Erdrotation auch noch vielfach Kurven infolge Kompensation des $W$ assers beschreiben werden, so daß wohl nicht zu hoch gegriffen wird, wenn wir die Wanderdauer verdoppeln. Alsdann wird meistens nur durchmischtes Wasser einfließen, da beim Sund Auslauf für ruhiges Wetter die Regel ist, in den Belten aber in 16 Tagen im Durchschnitt auf einen Sturm mit Einlauf reichlich $\mathrm{zu}$ rechnen ist.

Uber die Ursache der Salzgehaltsschwankungen in der Tiefe können wir uns erst an der Hand eines Materials von einer größeren Anzahl ron Stationen auslassen, wie es uns die internationalen Terminfahrten bieten. 
Tabelle 5. Monatsmittel des Salzgehaltes in ${ }^{0} 00$.

Drogden.

$0 \mathrm{~m}$.

\begin{tabular}{|c|c|c|c|c|c|c|c|c|c|c|c|c|c|c|c|c|c|}
\hline 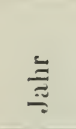 & 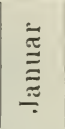 & 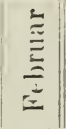 & $\stackrel{\stackrel{\bar{D}}{\Xi}}{\underline{\Xi}}$ & 䒿 & $\bar{\Xi}$ & $\bar{\Xi}$ & $\stackrel{\Xi}{\stackrel{\Xi}{\Xi}}$ & 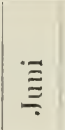 & 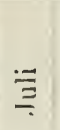 & 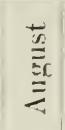 & $\underset{\Xi}{\stackrel{\Xi}{\Xi}}$ & 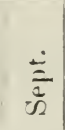 & $\frac{\frac{0}{0}}{\frac{ \pm}{5}}$ & 产 & 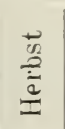 & $\stackrel{\dot{v}}{\vdots}$ & 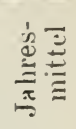 \\
\hline & & & & & & & & & & & & & & & & & \\
\hline & & & & $\begin{array}{r}10,8 \\
9,2\end{array}$ & $\begin{array}{r}9,6 \\
118\end{array}$ & & $\begin{array}{r}9,8 \\
10.2\end{array}$ & $\begin{array}{r}7,9 \\
11,2\end{array}$ & $\begin{array}{l}9,9 \\
8,7\end{array}$ & & & & & & & & 9 \\
\hline & & 12,7 & 12,8 & 8,6 & 12,4 & 8,1 & 9,7 & 8,3 & 9,9 & 9.2 & 9.1 & 10.6 & .0 & 4 & 10,3 & 13.2 & $10, \bar{x}$ \\
\hline & 1 & 9,3 & 11,3 & 13,4 & 8.2 & 7.9 & 9,8 & 9,1 & 9,6 & 9.6 & 9,4 & 9,2 & 10.3 & 15.6 & 11,7 & 11,7 & 10,4 \\
\hline & & 12,1 & 12,2 & 11,3 & 10,8 & 8,3 & 10,1 & 9,0 & 8.7 & 12,0 & 9.9 & 10,2 & 8,3 & & .4 & 0,3 & 10,3 \\
\hline & & 12 & 13,1 & 8,2 & 9,0 & 9 & 8,8 & 8,1 & 7,8 & $10, \overline{5}$ & 8,8 & 10,5 & 8,7 & 14 & &, 1 & 10,5 \\
\hline & & 10 & 11,3 & - & 9,2 & $9, \check{0}$ & 9,4 & 9,0 & 11,7 & 9,5 & 10,1 & 9,9 & 11,9 & & 10.9 & & 10,7 \\
\hline & & & 12.1 & 10,1 & 9,9 & 8,6 & $9 . \bar{\jmath}$ & 8,9 & 9,2 & 9,3 & 9,1 & 9,2 & 10,4 & & 10.7 & 12.9 & 10,4 \\
\hline 191 & 12 & 13 & 12.9 & 8,4 & 10.6 & 7.6 & 8.9 & 9.2 & 10.1 & 9.4 & 9.6 & 12.5 & $9 !$ & 14 & 12.3 & $\mid 11,3$ & 10,8 \\
\hline
\end{tabular}

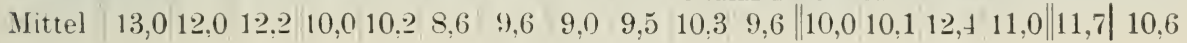
$6 \mathrm{~m}$ bis 31. Dezember 1908; $5 \mathrm{~m}$ rom 1. Januar 1909.

\begin{tabular}{|c|c|c|c|c|c|c|c|c|c|c|c|c|c|c|c|c|c|}
\hline$\stackrel{\Xi}{\Xi}$ & $\stackrel{\Xi}{\stackrel{\Xi}{\Xi}}$ & 竎 & $\stackrel{\stackrel{\mathscr{0}}{\Xi}}{ٍ}$ & $\stackrel{N}{\stackrel{N}{: ~}}$ & $\bar{\Xi}$ & $\stackrel{\Xi}{\equiv}$ & 导 & $\stackrel{\Xi}{\Xi}$ & $\Xi$ & 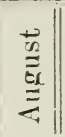 & 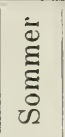 & 芯 & $\frac{\bar{d}}{\frac{\Delta}{0}}$ & $\frac{1}{2}$ & $\frac{\vec{n}}{\overrightarrow{0}}$ & $\stackrel{\text { อิ }}{\varrho}$ & 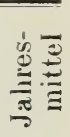 \\
\hline 1902 & 127 & 175 & 151 & 117 & 101 & 9. & 102 & 81 & $10=$ & 1) 0 & 105 & 10? & 100 & 11,0 & 111 & 15,9 & 113 \\
\hline $1904^{\circ}$ & 13,8 & 12,2 & 11,5 & 10,6 & 13,2 & 10,5 & 11,4 & 12.4 & 9.1 & 11.9 & 11,1 & 8,6 & 12.8 & 14,4 & 11.9 & 14,0 & 12,0 \\
\hline 1905 & 13,3 & 13,6 & 13.6 & 9,1 & 13,1 & 8,5 & 10,2 & 9,8 & 10,9 & 9,8 & 10,2 & 11,4 & 10.4 & 10,9 & 10.9 & 13,9 & 11,2 \\
\hline 1906 & 12,2 & 10.0 & 12,0 & 14,0 & 8.5 & 8,0 & 10.2 & $9 . \overline{5}$ & 10,1 & 10.1 & 9.9 & 9,6 & 12,3 & 17.6 & 13,2 & 12,9 & 11.2 \\
\hline 1907 & 13,4 & 12.9 & 13,1 & 11.8 & 11,1 & 8,6 & 10.5 & 9,6 & 8.9 & 12.7 & 10.4 & $10, \check{\partial}$ & 8,6 & 11,0 & 10.0 & 11,9 & 10.9 \\
\hline 1908 & 18,1 & 13.7 & 14,6 & 8,4 & 10,2 & 10,2 & 9,6 & 8,4 & 8,1 & 11,3 & 9.3 & 10,9 & 10,0 & 17,3 & 12,7 & 11,6 & $11 . \tilde{)}$ \\
\hline 1909 & 14,7 & 12,0 & 12,8 & - & 11.0 & 10.8 & 10.9 & 9,9 & 12.7 & 10,1 & 10.9 & 10,1 & 13,7 & 11,5 & 11.8 & 13,0 & 11.8 \\
\hline 1910 & 14,6 & 10,7 & 12,8 & 10,7 & 10,1 & 9,0 & 9.9 & 9,2 & 9,6 & 9,4 & 9.4 & 9,7 & 10,7 & 13.2 & 11.2 & 13,3 & 10,9 \\
\hline 1911 & 13.4 & 13,5 & 13.4 & 8.7 & 11,2 & 7.7 & 9,2 & 9,4 & 10,6 & 9,9 & 10.0 & 12.9 & 10.1 & 14.7 & 12,6 & 12,0 & 11,2 \\
\hline
\end{tabular}

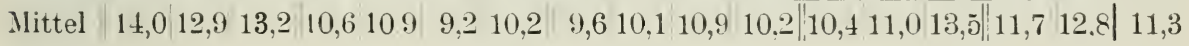

\section{Lappe Grund.}

$0 \mathrm{~m}$.

\begin{tabular}{|c|c|c|c|c|c|c|c|c|c|c|c|c|c|c|c|c|c|}
\hline$\stackrel{\Xi}{\Xi}$ & 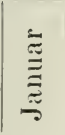 & 劳 & $\stackrel{\stackrel{\mathscr{U}}{\Xi}}{\stackrel{\Xi}{\Xi}}$ & 离 & $\overline{\bar{z}}$ & $\stackrel{\bar{\sigma}}{=}$ & 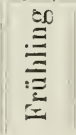 & 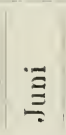 & $\fallingdotseq$ & 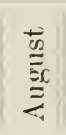 & 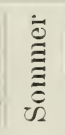 & $\overrightarrow{\vec{a}}$ & $\frac{\overline{0}}{\frac{0}{0}}$ & $\gtreqless$ & 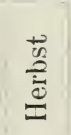 & $\stackrel{\text { ఏ }}{\varrho}$ & 苾 \\
\hline & & & & & & & & & & & & & & & & 14.5 & \\
\hline & 4,0 & 22.0 & 16,9 & 13,1 & 12.9 & 10,2 & 12,1 & & 12,3 & 15,4 & 12,4 & & 12.5 & & 13,2 & 9, & \\
\hline & 4,4 & 15,8 & 13.2 & 11. & 14,1 & 12,9 & 12.8 & 14,5 & 12,2 & & & & & & 14,0 & 16,5 & \\
\hline & & 16,2 & 16,1 & & 14,8 & 10,4 & 11,7 & 11 & 13,6 & & & & & 12.5 & 13,4 & 16.5 & 13,4 \\
\hline & & 12,1 & 14,4 & 16 , & 10 & 8,6 & 11,5 & & 11 & & & & & & 14.6 & 16.3 & 13, \\
\hline & & 14 & $15, \check{2}$ & & 11 & 10 & 12 & & & & & & & & & & 13 \\
\hline & & & 18,9 & 8,6 & 1 & 13,6 & 11.4 & & & & & & & & & & 14, \\
\hline & & 15 & 15.3 & - & 13,5 & 12,8 & 13,2 & & & 12,3 & & & & & & 16.1 & 14 \\
\hline & & 12 & $15, \check{\partial}$ & 11,8 & 12. & 10 & 11 & & & & & & & & & & 12, \\
\hline & & อ..4 & & & $1+?$ & 8.5 & 11.2 & & 20.0 & & & & & & & 14.3 & 13, \\
\hline
\end{tabular}

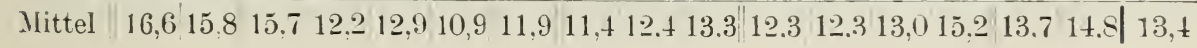




\section{Lappe Grund.}

Salzgehalt $\%$ (o)

$10 \mathrm{~m}$.

\begin{tabular}{|c|c|c|c|c|c|c|c|c|c|c|c|c|c|c|c|c|c|}
\hline 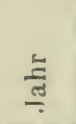 & 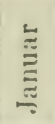 & 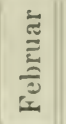 & 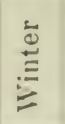 & 䒿 & 豆 & ت & $\underset{\Xi}{\stackrel{E}{\Xi}}$ & ఏ & $\equiv$ & 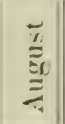 & 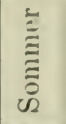 & $\underset{\tilde{C}}{\bar{c}}$ & $\frac{\frac{0}{0}}{\frac{0}{0}}$ & $\ddot{\bar{y}}$ & $\sum_{0}^{\frac{\pi}{d}}$ & $\stackrel{\text { c̀ }}{\stackrel{c}{a}}$ & 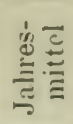 \\
\hline $\begin{array}{l}1 !(5) \cdot 2 \\
19003\end{array}$ & 2,2 & & 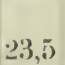 & & $17^{\circ}$ & & & 100 & 107 & & & & & & & & \\
\hline 1904 & 25,7 & 26,9 & 25.2 & 28.6 & 25,1 & 20,0 & 24,5 & 19,5 & 18,8 & 18,0 & 18,8 & 19,2 & 23 & 20,5 & 21,0 & 22,2 & 22,3 \\
\hline 1905 & 19,5 & 19.6 & 20,4 & $2,2,2$ & 22,4 & 17,$6 ;$ & $\because 0,7$ & 20,4 & 17,6 & 17,9 & 18,6 & 19,4 & 18,7 & 23,9 & 20,7 & 22,0 & 20,1 \\
\hline 1906 & 21,3 & 20.5 & 21,3 & 19,8 & $18, \overline{5}$ & 17,2 & 18,5 & 16,4 & 15.6 & 15,7 & 16,0 & 18,7 & 25 & 26,7 & 23,6 & 25,1 & 20,1 \\
\hline 1907 & 20 & 23,2 & 23,1 & & 21 & 18,0 & 20,2 & & 15 & & 17 & & & & 23,1 & & 21,2 \\
\hline 1908 & 26,2 & 26,1 & 27,1 & 24,0 & 27,0 & 24,0 & 25,0 & 21,9 & 21,0 & 19,1 & 20,7 & 20,1 & 23,7 & 22,9 & 22,2 & 24,2 & 23,4 \\
\hline $190 !$ & 23.5 & 20,5 & 22,7 & - & 23,7 & 20,4 & $2 \cdot 2,1$ & 18,9 & 19,5 & 18,6 & 19,0 & 18,7 & 23,8 & 20,8 & 21,1 & 23,6 & 21,1 \\
\hline 1910 & 22,1 & 22,7 & $2 \cdot 2,6$ & $17, \overline{0}$ & 18,0 & 18,1 & 17,9 & 19,0 & 16,9 & 17,4 & 17,8 & 21,2 & 22,9 & 25.5 & 23,2 & 24,4 & 20,5 \\
\hline 1911 & 21,6 & 23.0 & 233,0 , & & 18.7 & 19.1 & 19.7 & 17,9 & 16.8 & & 179 & 19,5 & & 21,0 & 20,4 & $28,1 \mid$ & 20,5 \\
\hline
\end{tabular}

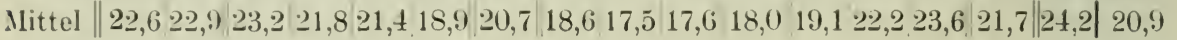

$15 \mathrm{~m}$.

\begin{tabular}{|c|c|c|c|c|c|c|c|c|c|c|c|c|c|c|c|c|c|}
\hline$\stackrel{\Xi}{ٍ}$ & 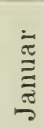 & 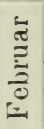 & $\stackrel{\stackrel{\Phi}{\Xi}}{\Xi}$ & لِّ & 冚 & $\underset{\Xi}{\bar{\Xi}}$ & 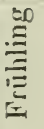 & $\stackrel{\Xi}{\Xi}$ & 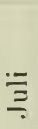 & 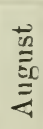 & 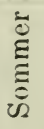 & 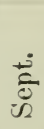 & $\begin{array}{l}\frac{0}{0} \\
\frac{0}{0} \\
\frac{ \pm}{0}\end{array}$ & రั & 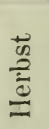 & $\stackrel{\text { ¿ }}{\varrho}$ & 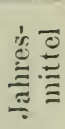 \\
\hline
\end{tabular}

1902

\begin{tabular}{lllll|llllllllllll|llllllllll}
1903 & 25,8 & 25,0 & 26,4 & 27,4 & 22,7 & 24,0 & 24,7 & 26,4 & 23,5 & 19,1 & 23,0 & 21,2 & 27,7 & 25,2 & 24,7 & 28,5 & 24 & 24 \\
1904 & 31,5 & 30.2 & 30,1 & 31,3 & 29,4 & 25,7 & 28,8 & 24,0 & 21,1 & 21,1 & 22,1 & 25,1 & 29,3 & 24,0 & 26.1 & 25,5 & 26 & 5
\end{tabular}

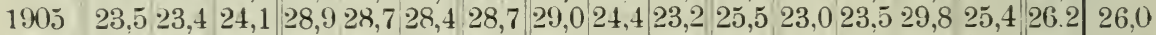

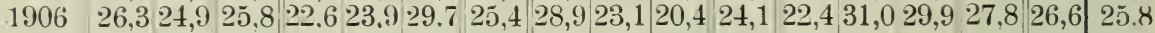

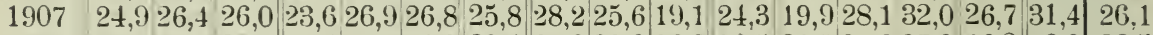

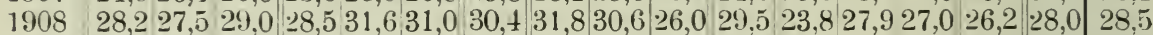

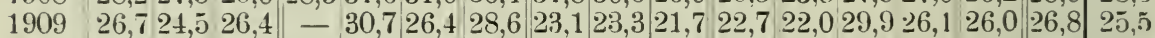

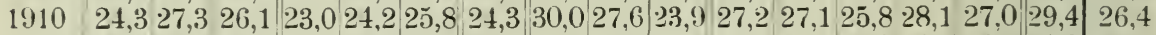

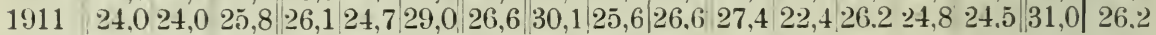

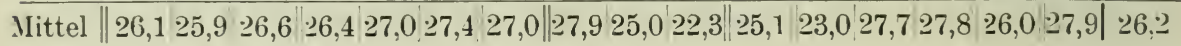

$20 \mathrm{~m}$.

\begin{tabular}{|c|c|c|c|c|c|c|c|c|c|c|c|c|c|c|c|c|c|}
\hline 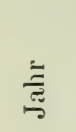 & 竎 & 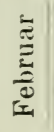 & 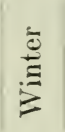 & $\stackrel{\text { 荧 }}{=}$ & $\overline{\bar{E}}$ & $\stackrel{\bar{E}}{\bar{z}}$ & $\stackrel{\infty}{\stackrel{\infty}{\Xi}}$ & $\underset{\Xi}{\Xi}$ & $\fallingdotseq$ & 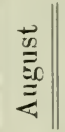 & 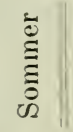 & $\stackrel{\vec{\Xi}}{\breve{\Xi}}$ & & $\begin{array}{l}3 \\
z\end{array}$ & $\frac{\overrightarrow{0}}{\grave{0}}$ & $\stackrel{8}{0}$ & 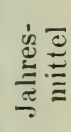 \\
\hline 190 & & & & & & & & & & & & & & 30,7 & & 30,7 & \\
\hline 03 & 28,4 & 27,0 & 28,7 & 29,6 & 27,5 & 29,3 & 28,8 & 30.2 & 30,0 & 23,5 & 27,9 & 24,3 & 31,1 & 29,8 & 28.4 & 30,3 & 28,4 \\
\hline 1904 & 32,7 & 1,8 & 31,6 & 32,1 & 31,8 & 30,9 & 31,6 & $\gtrless 9,9$ & 27,3 & 27,8 & § 8,3 & & & 27,9 & 29,8 & 28,5 & 30 \\
\hline & 25,8 & 25,5 & 26,6 & 31,0 & 31,9 & 31,7 & 31,5 & 32 & 31 & 29,7 & 31,1 & 27 & & & 26,5 & 29.1 & 29,8 \\
\hline 1906 & 29 & 28,6 & & 2 & & & 29,1 & & & 27,6 & 30 & & & 32,0 & 305 & 28,0 & 29,8 \\
\hline 1907 & 27 & 29 & 28 & 27 & 31 & 31,5 & 30,0 & 32 & 31 & 24,8 & 29,8 & 23,7 & 30,0 & 32.6 & 28,8 & 32,1 & 29.5 \\
\hline 1908 & 29, & 28,1 & 30,0 & 29,9 & 32,4 & 33,0 & 31.8 & 33,0 & 32,8 & 32,0 & 32.6 & 29,0 & 30,6 & 30,6 & 30,1 & 29,9 & 30,9 \\
\hline 1909 & 29 & 28,1 & 29,3 & - & 32,3 & 31,1 & 31,7 & 30,7 & 28,9 & 23.9 & 27,8 & 25,5 & 30.7 & 29,5 & 28,6 & 28,7 & 29.0 \\
\hline 1910 & 27,0 & 29,1 & 28,3 & 29,4 & ⒏9 & 31,1 & 29,8 & 32, & 31,6 & 30,9 & & & & 30,1 & 30,2 & 31,4 & 30,2 \\
\hline 191 & & & & & & & 299 & & & 308 & 317 & & & 283 & 28,5 & 31,6 & 29 . \\
\hline
\end{tabular}

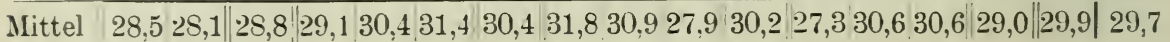




\section{Lappe Grund.}

Salzgehalt $\%$.

$23 \mathrm{~m}$.

\begin{tabular}{|c|c|c|c|c|c|c|c|c|c|c|c|c|c|c|c|c|c|}
\hline$\underset{\Xi}{\leftrightarrows}$ & 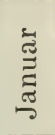 & 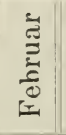 & 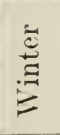 & $\stackrel{N}{\stackrel{N}{*}}$ & $\overrightarrow{\bar{a}}$ & $\stackrel{\pi}{\pi}$ & : & $\stackrel{\Xi \Xi}{\Xi}$ & בี & 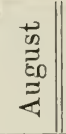 & 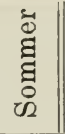 & $\begin{array}{l}\frac{3}{d} \\
\text { di }\end{array}$ & 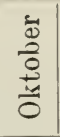 & है & $\frac{0}{0}$ & 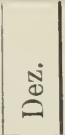 & 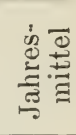 \\
\hline $\begin{array}{l}902 \\
903\end{array}$ & 29,4 & 28,2 & 29,6 & 30,2 & 29,2 & 30,6 & 30,0 & 31,7 & 31,7 & 27,5 & 30,3 & 26,3 & 32,1 & $\begin{array}{l}32,1 \\
31,0\end{array}$ & 29,8 & $\begin{array}{l}31,3 \\
31,0\end{array}$ & 29,9 \\
\hline 1904 & 3,1 & 32,4 & 32.2 & 32,7 & 32.5 & 32,4 & 32,5 & & 30,2 & 30,8 & 30,9 & 31,1 & 32, & 30,1 & 31,2 & 29,9 & 31,6 \\
\hline 905 & 27,6 & 26,8 & 28,1 & 31,8 & 32,8 & 32,4 & 32,3 & 32 & 32,9 & 31,5 & 32,4 & 30 , & 31,6 & 33,0 & 31,7 & 30,7 & 31,2 \\
\hline 906 & 30,6 & 30,1 & 30,5 & 27,0 & 30,1 & 33,3 & 30,1 & 33,5 & 33,0 & 30,9 & 32,5 & 30, & 32,7 & 32,4 & 31.9 & 29,0 & 31,1 \\
\hline .907 & 28 & 30,1 & 29,0 & 29,1 & 31,8 & 32,4 & 31,1 & 33,1 & 33,0 & 29,2 & 31,8 & 27, & 31,4 & 32,8 & 30,5 & $32 ; 5$ & 30,9 \\
\hline 19 & 30,4 & 28,3 & 30,4 & 30,3 & 32,9 & 33,3 & 32,2 & 33,4 & 33,2 & 32,8 & 33,1 & 31,3 & 31,5 & 31,2 & 31,3 & 31,3 & 31 , \\
\hline 1909 & 31,2 & 29,7 & 30,7 & - & 32,7 & 32,2 & 32,5 & 32,0 & 31,5 & 26,5 & 30,0 & 28,2 & 31,1 & 31,1 & 30,1 & 29,7 & 0,5 \\
\hline 1910 & 28,8 & 29,8 & 29,4 & 30,8 & 30,9 & 31,9 & 31,2 & 0 & 32,1 & 31,6 & 32,2 & 31,8 & 30,8 & 31,4 & 31,3 & 32,5 & 1 , \\
\hline 1911 & & 27,0 & & & 29 & 32.0 & 30,3 & 32 & 32,8 & & 32,4 & & & & 30, & 32,0 & 0,6 \\
\hline te & & 9 9 & & & & & & & & & & & & & & 309 & \\
\hline
\end{tabular}

Schultz Grund.

Salzgehalt $\%$.

$0 \mathrm{~m}$.

\begin{tabular}{|c|c|c|c|c|c|c|c|c|c|c|c|c|c|c|c|c|c|}
\hline$\stackrel{\Xi}{\Xi}$ & 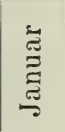 & 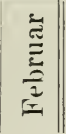 & $\stackrel{\stackrel{\Xi}{\Xi}}{\stackrel{\Xi}{\Xi}}$ & 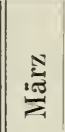 & $\overline{\bar{z}}$ & $\vec{\pi}$ & 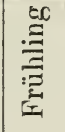 & $\stackrel{\Xi}{\Xi}$ & $引$ & 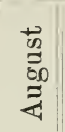 & . & $\begin{array}{l}\stackrel{0}{0} \\
\dot{0} \\
\ddot{\Omega}\end{array}$ & $\begin{array}{l}\tilde{0} \\
\frac{0}{0} \\
\frac{0}{ \pm 0} \\
0\end{array}$ & 官 & 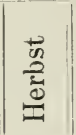 & ఏ̊ & 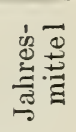 \\
\hline & 1,1 & 24,9 & 21.5 & & 19 & 12,4 & 16.6 & 12,3 & 14,0 & - & 13,2 & 17,8 & 15,5 & & 17,2 & & 17,3 \\
\hline & & 21,1 & 18,1 & & 19 & 20,5 & 18,9 & 16,8 & 19,7 & 18,0 & & & & 22,6 & 18,4 & 24,8 & 19,1 \\
\hline & 22 & 21,2 & 22,8 & 12,8 & 18,0 & 16,5 & 15,8 & 13, & 16,4 & 18,0 & 15,9 & & & 15,8 & 17,5 & 22,4 & 17 \\
\hline & & 19,5 & 20,9 & 20 & 13 & 11,3 & 15,2 & 13 & 15 & 16,4 & 15,1 & & & 20,3 & 18,6 & 22,5 & 17 \\
\hline & 20 & 19 & 21,0 & 230 & 14 & 16,4 & 18,1 & 15 & 18 & 20,2 & 17,8 & & & 1 & 15,4 & 23,0 & 18, \\
\hline & 26,5 & 28,2 & 25,9 & 13,6 & 14,9 & 15 & 15,8 & & & & & & & & & 23,4 & 19 \\
\hline & 21 , & 21,1 & 22,0 & - & 17.7 & 19 & 18,4 & & & & & & & & & 21 & 19,2 \\
\hline & & & 21,4 & 15,2 & 15 & 15 & 15,6 & & & & 15 & & & & 19,2 & 19,0 & 17,8 \\
\hline & & & 21,8 & & 166 & & 16 & & & 15 & 14 & & & & 20,7 & 17,5 & 18,3 \\
\hline
\end{tabular}

Mittel || 21,8|22,0|21,7||17,3|16,8|16,1||16,8||14,8 $16,8|17,9||16,3||17,7| 16,9|19,3||18,2||21,3| 18,2$

$10 \mathrm{~m}$.

\begin{tabular}{|c|c|c|c|c|c|c|c|c|c|c|c|c|c|c|c|c|c|}
\hline 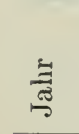 & 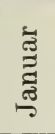 & 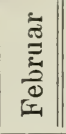 & $\begin{array}{l}\stackrel{\vec{D}}{\leftrightarrows} \\
\stackrel{\Xi}{=}\end{array}$ & 吢 & $\overline{\bar{L}}$ & 胥 & 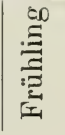 & $\stackrel{\Xi}{\Xi}$ & $\Xi$ & 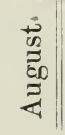 & 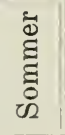 & 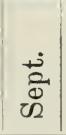 & $\begin{array}{l}\frac{0}{0} \\
\frac{0}{0} \\
\frac{7}{0}\end{array}$ & हे & 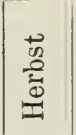 & 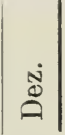 & 总 \\
\hline 03 & 3,4 & 25,7 & 23,2 & 19,8 & 20,8 & 15,5 & 18,7 & 16,7 & 16,5 & 19,4 & 17,5 & 19, & 17,8 & & 18,9 & & \\
\hline 0 & & 2,2 & $20, \tilde{0}$ & 20,3 & 22.3 & 22,7 & 21,8 & 20,6 & & 20,3 & 20,6 & & 18,6 & 23,0 & 19,7 & 25,5 & 21 \\
\hline 90 & 23 & 21,8 & 23.5 & 15,0 & 19,3 & 18,4 & 17.6 & 18, & 17, & 19,3 & 18,3 & 19,5 & 19,2 & 17,6 & 18,8 & 23,1 & 19 \\
\hline ( & 22,7 & 21,0 & 22,3 & 21,5 & 16,2 & 16,4 & 18,0 & 16,1 & 18 & 18,1 & 17,5 & 19 & & 1,8 & 20,0 & 24,1 & \\
\hline & & 21,7 & 22,7 & 23 & 17 & 18 & 19,9 & 17 & 2 & 20,7 & 19,6 & 20,5 & & 3,4 & $\mid 18,0$ & 25,7 & \\
\hline & 27,0 & 28,6 & 27,1 & 16,4 & 19,2 & 21,1 & 18,9 & 19 & & 20,2 & 9,6 & & & & 21,2 & & \\
\hline & & 22,5 & 23.4 & - & 19,9 & 22,1 & 21,0 & & & 22,2 & & & & & 20,8 & 22, & 21, \\
\hline & & 21,2 & 22,9 & 19,2 & 18 & & & & & & & & & & $\mid 21,3$ & 22,1 & 20 \\
\hline & & 4,2 & 23,3 & & 17, & 18,0 & & & & & & & & & $|22,2|$ & $20,9 \mid$ & 20 \\
\hline
\end{tabular}

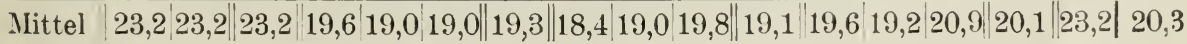

Spethmann, Ozeanographie. Hzdrogr. Suppl. z. V. Bd. 


\section{Sichultz tirind.}

Sialzgehalt $\%$ o.

$15 \mathrm{~m}$.

\begin{tabular}{|c|c|c|c|c|c|c|c|c|c|c|c|c|c|c|c|c|c|}
\hline$\stackrel{\Xi}{\Xi}$ & $\underset{\Xi}{\Xi}$ & है & $\stackrel{\stackrel{\Xi}{\Xi}}{=}$ & . & 总 & $\stackrel{\Xi}{=}$ & 永 & $\stackrel{\Xi}{\Xi}$ & 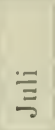 & 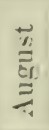 & 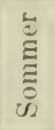 & $\stackrel{\vec{\Xi}}{\vec{U}}$ & & \% & 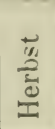 & 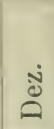 & 它离 \\
\hline & & & & & & & & & & & & & & & & (2) & \\
\hline
\end{tabular}

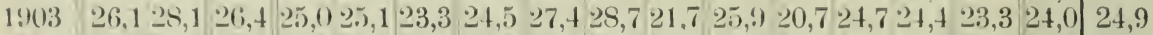

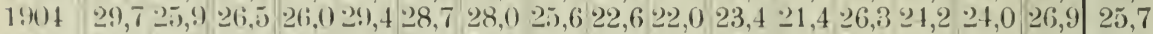

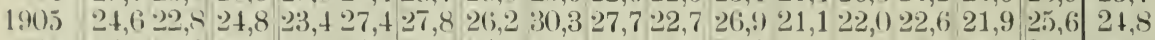

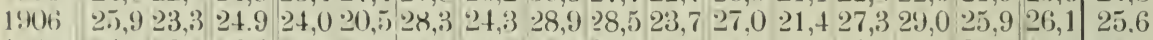

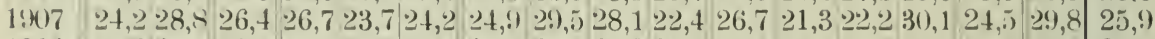

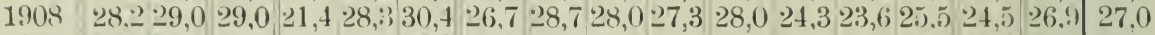

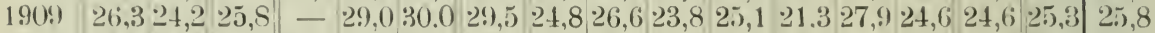

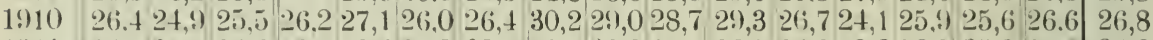

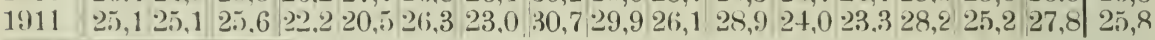

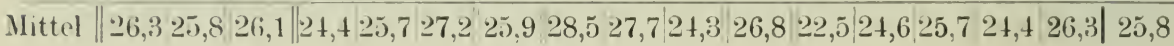

$20 \mathrm{~m}$.

\begin{tabular}{|c|c|c|c|c|c|c|c|c|c|c|c|c|c|c|c|c|c|}
\hline$\stackrel{\Xi}{\leftrightarrows}$ & 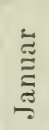 & $\frac{\ddot{\Xi}}{\tilde{\Xi}}$ & $\begin{array}{l}\bar{\Xi} \\
\vdots \\
\vdots\end{array}$ & 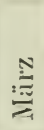 & $\overline{\bar{a}}$ & $\stackrel{\vec{J}}{\bar{z}}$ & $\stackrel{8}{\stackrel{B}{\Xi}}$ & $\stackrel{\Xi}{\Xi}$ & 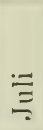 & 崖 & 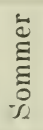 & $\underset{\widetilde{\sigma}}{\stackrel{\leftrightarrows}{\leftrightarrows}}$ & $\frac{\dot{0}}{\frac{0}{0}}$ & में & 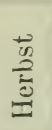 & 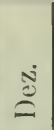 & 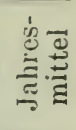 \\
\hline
\end{tabular}

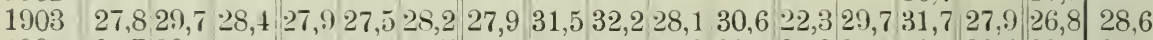

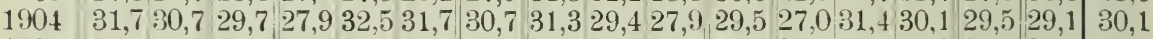

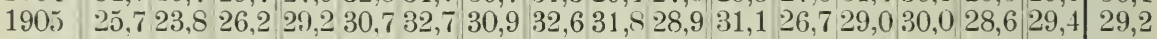

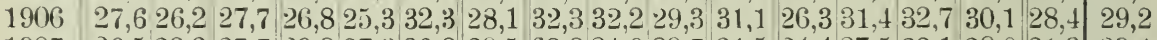

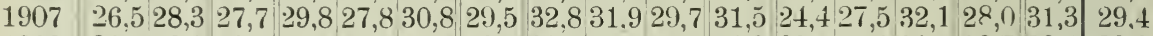

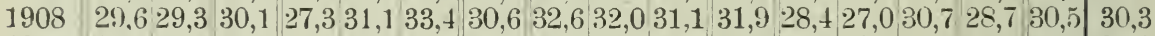

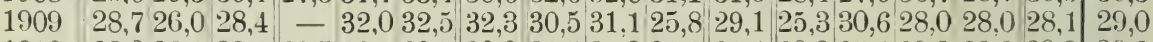

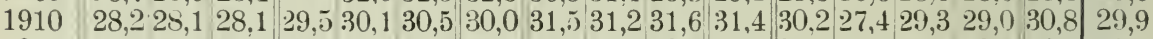

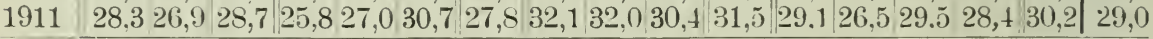

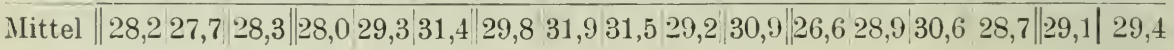

$26 \mathrm{~m}$.

\begin{tabular}{|c|c|c|c|c|c|c|c|c|c|c|c|c|c|c|c|c|c|}
\hline$\stackrel{\Xi}{\Xi}$ & 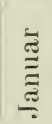 & $\frac{\vec{\Xi}}{\tilde{\Xi}}$ & $\begin{array}{l}\stackrel{\mathscr{0}}{\stackrel{\Xi}{\Xi}} \\
\stackrel{\Xi}{=}\end{array}$ & 胥 & 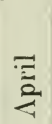 & $\overline{\mathrm{J}}$ & 吾 & $\Xi$ & 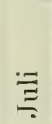 & 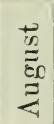 & 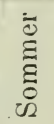 & 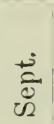 & $\frac{\dot{0}}{\frac{0}{0}}$ & 官 & 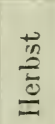 & $\stackrel{\dot{0}}{\stackrel{0}{0}}$ & 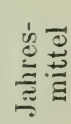 \\
\hline 1902 & & & & & & & & & & & & & & 31.8 & & 28,8 & \\
\hline
\end{tabular}

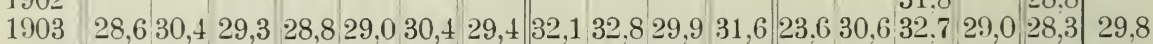

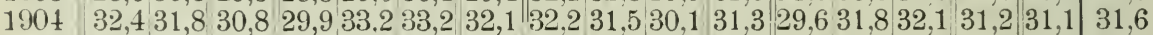

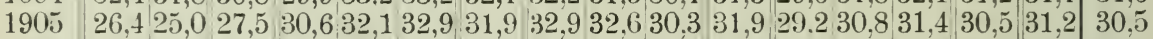

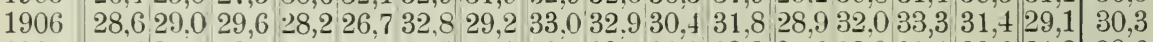

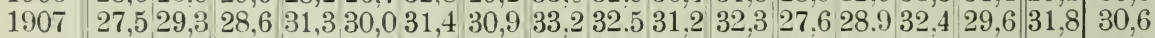

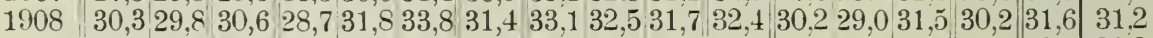

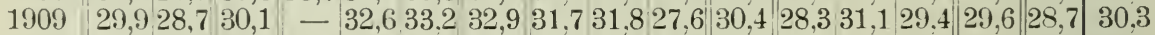

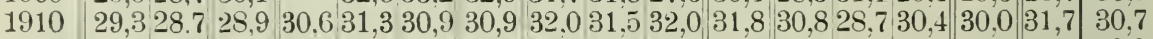

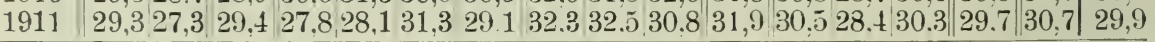

Mittel || 29,1 28,9 29,4 $29,5 \mid 30,5$ 32,2||30,9||32,4 32,3 30,4 $31,7 \cdot 28,7|30,131,7| 30,1 \quad 30,3 \mid 30,5$ 


\section{Gjedser Rer.}

Salzgehalt $\%$.

$0 \mathrm{~m}$.

\begin{tabular}{|c|c|c|c|c|c|c|c|c|c|c|c|c|c|c|c|c|c|}
\hline$\stackrel{\tilde{3}}{\stackrel{\Xi}{\leftrightarrows}}$ & $\stackrel{\Xi}{ٍ ٍ ~}$ & 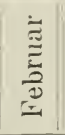 & $\stackrel{\stackrel{\Xi}{\Xi}}{\stackrel{\Xi}{=}}$ & $\stackrel{\text { : }}{\stackrel{N}{*}}$ & $\overline{\bar{g}}$ & 芸 & 竝 & $\Xi$ & $\overline{\bar{\Xi}}$ & $\stackrel{\substack{2 \\
3}}{3}$ & 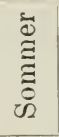 & $\begin{array}{l}\overrightarrow{0} \\
0 \\
\text { D. }\end{array}$ & $\frac{5}{0}$ & 3 & $\begin{array}{l}\vec{w} \\
\stackrel{\vec{w}}{\vec{d}} \\
\stackrel{\underline{\omega}}{=}\end{array}$ & ڤ̊ & 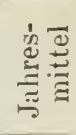 \\
\hline & & & & & & & & & & & & & & & 9 & & 9 \\
\hline 190 & & 10,2 & & 8,1 & 10,4 & 9,0 & 9,2 & & 8,2 & 10,1 & 9,4 & 7,8 & 10,6 & 13,2 & 10,5 & 13,1 & 10,1 \\
\hline $190 \tilde{~}$ & & 13,2 & 12,9 & 7,9 & $10, \tilde{\partial}$ & 7,8 & 8,7 & 7, & 8,6 & 8,2 & 8,1 & 9,9 & 10,0 & 8,7 & 9,5 & 12,3 & 9,8 \\
\hline 1906 & 10,3 & 9,8 & $10, \mathrm{~S}$ & 12,3 & 7,7 & 7,2 & 9,1 & 7, & 9,2 & 9,2 & 8,7 & 8,7 & 9,8 & 12,7 & 10,4 & 13,3 & 9 , \\
\hline 1907 & & 11,3 & 11,9 & 10,9 & 9,1 & $8, \overline{3}$ & 9,5 & 8,9 & 8,8 & 10,3 & 9,3 & 8,9 & 8,3 & 8,7 & 8,6 & 11,6 & 9,7 \\
\hline 1908 & 15,2 & 13,3 & 13,4 & 8,2 & 9,5 & 9,2 & 9,0 & 8,2 & 8,1 & 9,8 & 8,7 & 11,9 & 9,0 & 13,1 & 11,3 & 11,4 & 10,6 \\
\hline 1909 & 12,5 & 10,8 & 11,6 & - & 9,5 & 9,4 & 9,5 & 8,6 & 9,8 & 9,8 & 9,4 & 9,4 & 10,7 & 11,9 & 10,7 & 10,7 & 10,3 \\
\hline & & 9 & 11. & 9,4 & 9,9 & 8,5 & 9,3 & 8 & 10,2 & 9,3 & 9,4 & 9,4 & 10,2 & 13,3 & 11,0 & 11,2 & 10,3 \\
\hline 1911 & 13,6 & 11,9 & 12,2 & 9,7 & 10,4 & 8,0 & 9,4 & 8,7 & 9,8 & 9,2 & 9,2 & 10,3 & 10, & 13,2 & 11,2 & || $10,7 \mid$ & 10,5 \\
\hline & & & & & & & & & 90 & & 0 & & & 11 & 10,3 & 11,5 & 1 \\
\hline
\end{tabular}

$5 \mathrm{~m}$

\begin{tabular}{|c|c|c|c|c|c|c|c|c|c|c|c|c|c|c|c|c|c|}
\hline$\stackrel{\Xi}{\Xi}$ & $\stackrel{\Xi}{\Xi}$ & $\frac{\mathfrak{\Xi}}{\stackrel{\Xi}{\Xi}}$ & $\underset{\Xi}{\stackrel{\Xi}{\Xi}}$ & $\stackrel{\Xi}{:}$ & 离 & 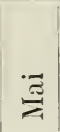 & 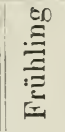 & $\Xi$ & 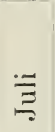 & 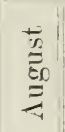 & 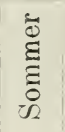 & 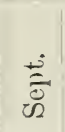 & $\frac{\dot{0}}{\frac{0}{0}}$ & 宛 & 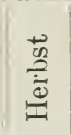 & 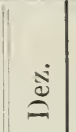 & $\frac{\dot{d}}{\stackrel{\Xi}{J}}$ \\
\hline & 115 & 161 & 13.1 & 9.6 & 10,1 & 7.8 & 9,2 & 7,6 & 8.5 & 10.8 & 9.0 & 9,0 & 8,6 & $\begin{array}{l}10,4 \\
11,9\end{array}$ & 9.8 & & 10, \\
\hline & & & & & 10 & 9,4 & 9.5 & 10.3 & & & 9.9 & 8,0 & 11,3 & 13.3 & 10,9 & 13,5 & \\
\hline 1905 & & 13.6 & 13,3 & 7.9 & 11,8 & 8.6 & 9,4 & 7,6 & 9,6 & 8.3 & 8.5 & 10,3 & 10,2 & 8,8 & 9,8 & 12,6 & \\
\hline 1906 & 10 & 10,0 & 11,1 & 12,8 & 7,9 & 7,4 & 9,4 & 8,6 & 9,7 & 9,6 & 9,3 & 9.0 & 10,1 & 12,9 & 10,7 & 13,6 & 10 , \\
\hline & & 1 & 12,2 & 11.4 & 9.2 & 8 , & 9 & 8 & 8,9 & 10,6 & 9.5 & 9,0 & 8.4 & 8,8 & 8.7 & 11,8 & \\
\hline & & 13 & 13.7 & 8.2 & 9.6 & 9.5 & 9. & 8.3 & 8.2 & 9.9 & 8.8 & 12.1 & 9,1 & 13.2 & 11.5 & 12,1 & \\
\hline & & & 12.1 & - & 10.3 & 10.3 & 10.3 & 9.3 & 10,4 & 10.6 & 10,1 & 9.5 & 11,4 & 124 & 11.1 & 10,9 & \\
\hline & & 10 & 11,7 & 9.8 & 10,1 & 8.9 & 9 & 8,8 & 10,7 & 9,5 & 9.7 & 9.6 & & 13.5 & 1 & 11,7 & \\
\hline 1911 & 13 & 12.1 & 12,6 & 10.2 & 10,8 & 8.1 & 9,7 & 9,1 & 0,1 & 9.6 & 9.9 & 10.5 & & 13,3 & 11,4 & & \\
\hline ittel & 12.7 & 12,1 & 12.2 & 9.8 & 10,1 & 8.7 & \begin{tabular}{|l}
9,6 \\
a
\end{tabular} & 8,7 & & 10.0 & 9,4 & 9.7 & 10,0 & 11,7 & $\| 10,6$ & 11,8 & \\
\hline
\end{tabular}

$11 \mathrm{~m}$

\begin{tabular}{|c|c|c|c|c|c|c|c|c|c|c|c|c|c|c|c|c|c|}
\hline$\stackrel{\Xi}{\Xi}$ & $\stackrel{\Xi}{\Xi}$ & 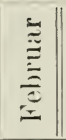 & 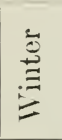 & $\stackrel{N}{\stackrel{N}{*}}$ & 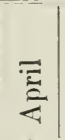 & 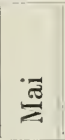 & 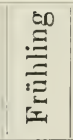 & $\stackrel{\Xi}{\Xi}$ & ב & $\begin{array}{l}\stackrel{+}{02} \\
\stackrel{3}{8} \\
\stackrel{\Xi}{u}\end{array}$ & 离 & $\underset{0}{\stackrel{0}{0}}$ & $\frac{\frac{5}{0}}{\frac{0}{0}}$ & 8 & $\frac{\vec{w}}{\stackrel{\overrightarrow{0}}{\overrightarrow{0}}}$ & $\stackrel{\dot{D}}{0}$ & 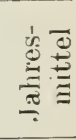 \\
\hline $\begin{array}{l}1902 \\
1903\end{array}$ & 12,5 & 16,5 & 13.7 & 11.6 & 11.8 & 8.8 & 10,7 & 8.6 & 10.5 & 12.7 & 10,6 & 9.5 & 8.9 & $\begin{array}{l}11,3 \\
13,2\end{array}$ & 10.5 & $\begin{array}{r}12,0 \\
9.2\end{array}$ & 11.2 \\
\hline 1904 & 12.9 & & & 10.3 & 12.9 & 11.5 & 11,3 & 12,8 & 11.5 & 12,0 & 12.1 & 8.5 & 13.0 & 13,7 & 11.7 & 14.4 & 12.1 \\
\hline 1905 & 13,2 & 14,0 & 13,9 & 8,2 & 13.3 & 11,2 & 10,2 & 8.5 & 12.0 & 9,3 & 9.9 & 10.9 & 10.9 & 9,0 & 10,3 & 13,1 & 11.1 \\
\hline 1906 & 11 & 10,3 & 11,6 & 13,5 & 9,1 & 8.1 & 10,2 & 9,9 & 10,9 & 11,2 & 10,7 & 10,0 & 11,1 & 13.5 & 11.5 & 15,1 & 11,1 \\
\hline 1907 & 13,7 & 11,8 & 13,5 & 13,4 & 10,4 & 10,1 & 11,3 & 9.8 & 9,8 & 11,3 & 10,3 & 10,0 & 8.7 & 9,5 & 9,4 & 13,0 & 11,0 \\
\hline 1908 & 16,6 & 15,6 & 15,1 & 8,7 & 10,8 & 11,5 & 10,3 & 9.6 & 9.0 & 11.7 & 10.1 & 13.0 & 10,9 & 14,3 & 12,7 & 13,6 & 12,1 \\
\hline 1909 & 14, & 14,0 & 14,1 & 1 & 12,7 & 12,1 & 12,4 & 10.8 & 12,4 & 12,0 & 11,7 & 10,0 & 13.2 & 13,2 & 12,1 & 11,3 & 12.4 \\
\hline 1910 & 14,3 & 11,2 & 12,3 & 11,5 & 11,8 & 10,1 & 11,1 & 9,6 & 11,9 & 10,1 & 10.5 & 10,5 & 12.2 & 14,2 & 12,3 & 12,4 & 11,7 \\
\hline 1911 & & & 13.6 & 10,9 & 12,1 & 9.2 & 10,7 & 10.9 & 11,9 & & 11,7 & 13,0 & 10,9 & 13,7 & 12,5 & $|11,7|$ & 12,1 \\
\hline ittel & 138 & 129 & 9 9 & 110 & 116 & 10.3 & 10,9 & 10,1 & 111 & 114 & 10,8 & 10,6 & 11.1 & 124 & 114 & 12.7 & 116 \\
\hline
\end{tabular}




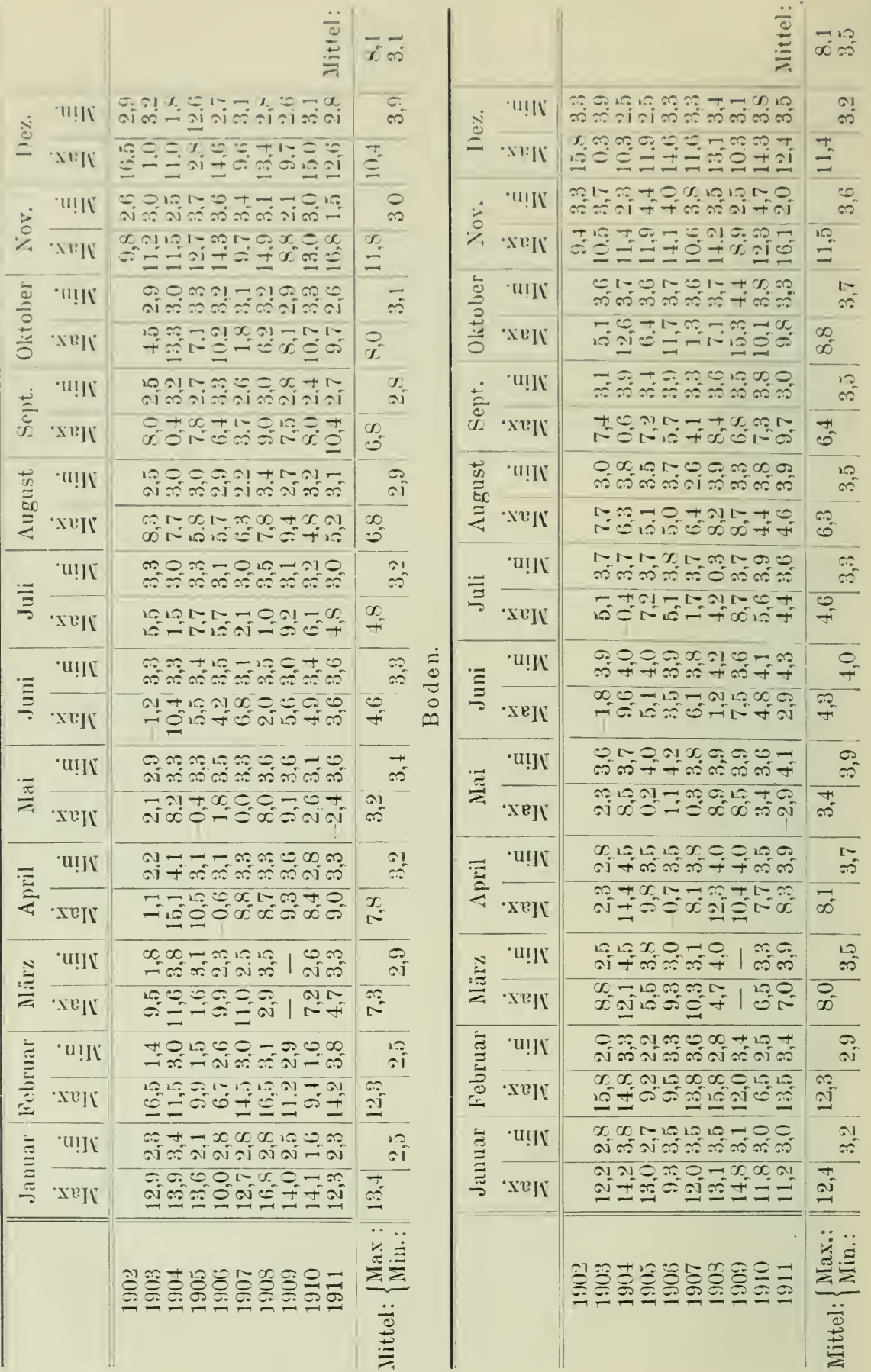




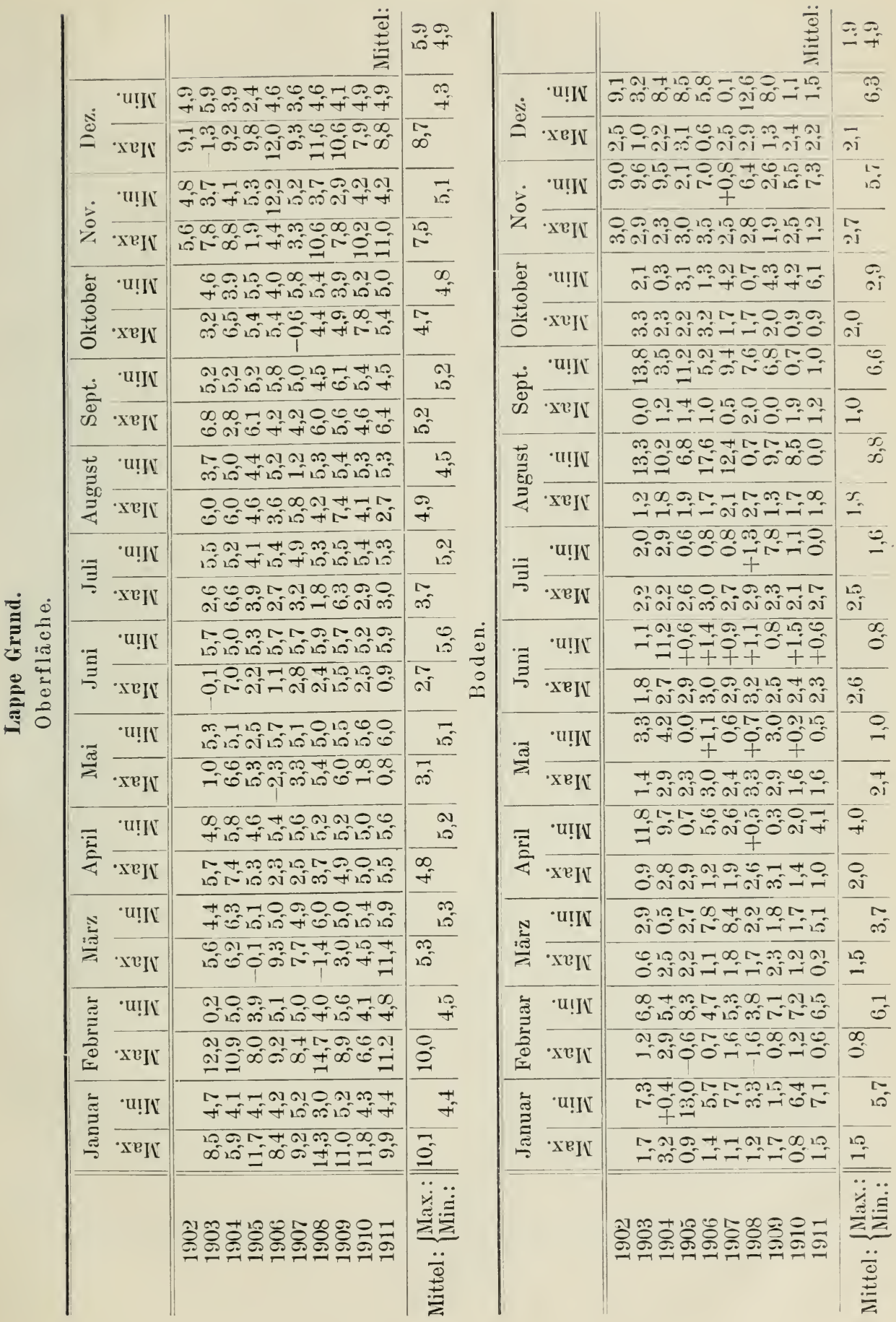




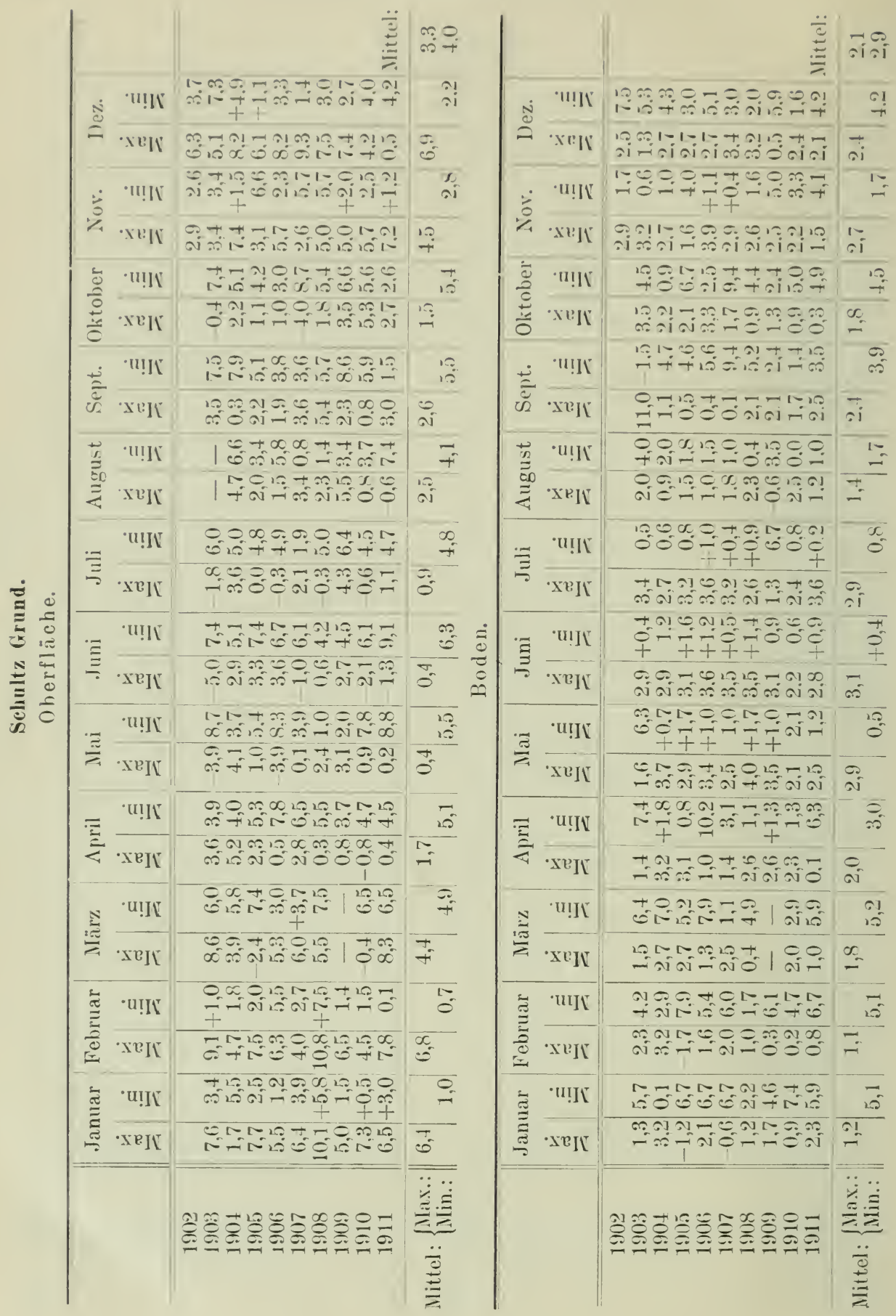




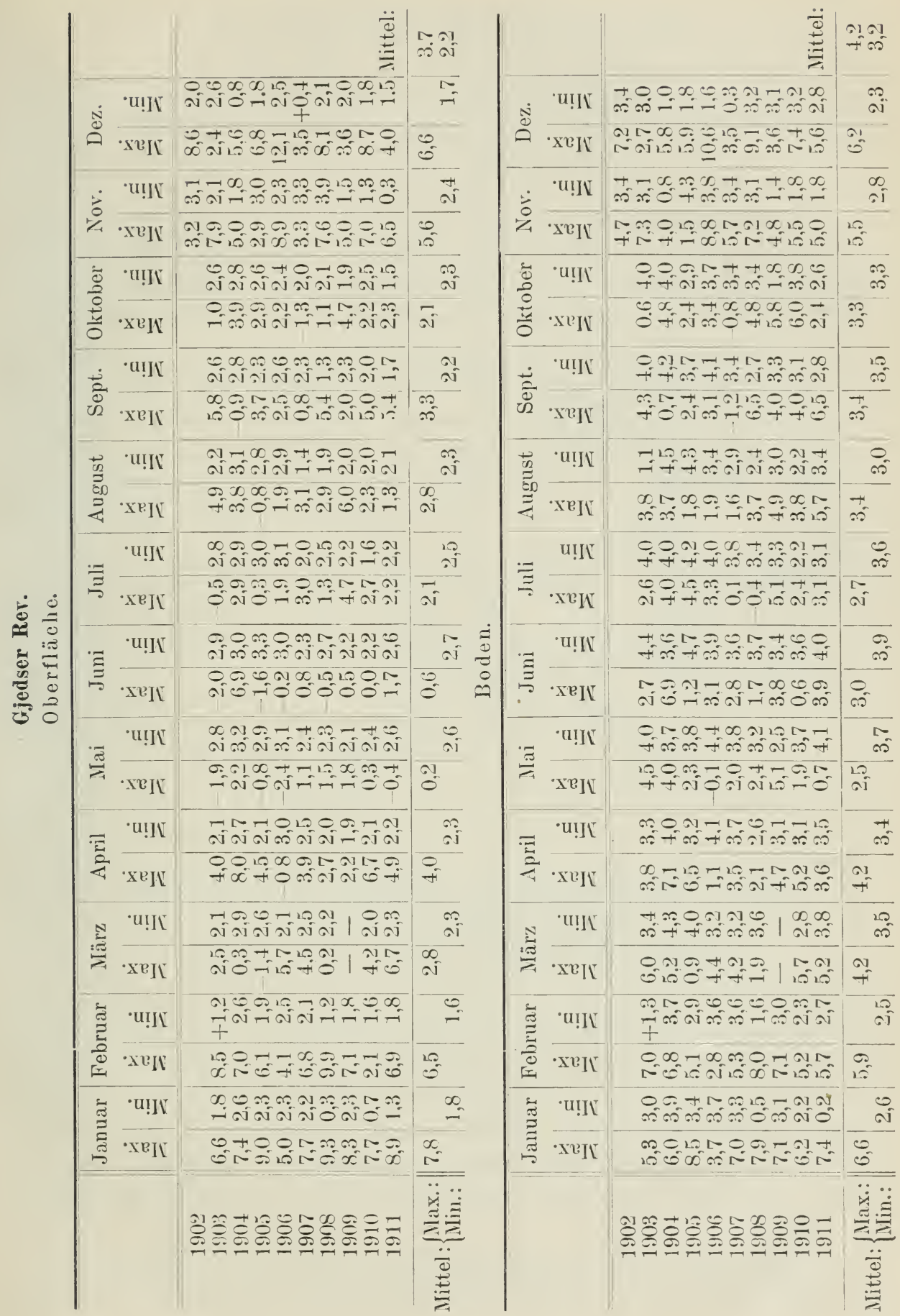




\section{Schwankungen des Salzgehaltes II.}

Die Ciewinnung der Beobachtungen S. 7:2. - Die Genangkeit der Beobarhlungen S. 74. - 1)er Gang des Salzgehaltes rom südlichen Kattegat \%ur Arkonasee S. 75. Die Extreme am Boden S. SO. - Was atlantische Wasser S. 82. - Die Extreme an der Oberfläche S. 84. - Die Schwankungen zwischen Boden und Oberfläche S. 85. Tabellen S. 86 .

Die Beobachtungen auf den internationalen Terminfahrten sind seit August oder November 1902 viermal im Jahre ausgeführt, in der Regel Anfang Februar, Mai, August und November. Trotzdem sie noch heute erfolgen, seit 1907 aber mit einer nicht unwesentlichen Modi-

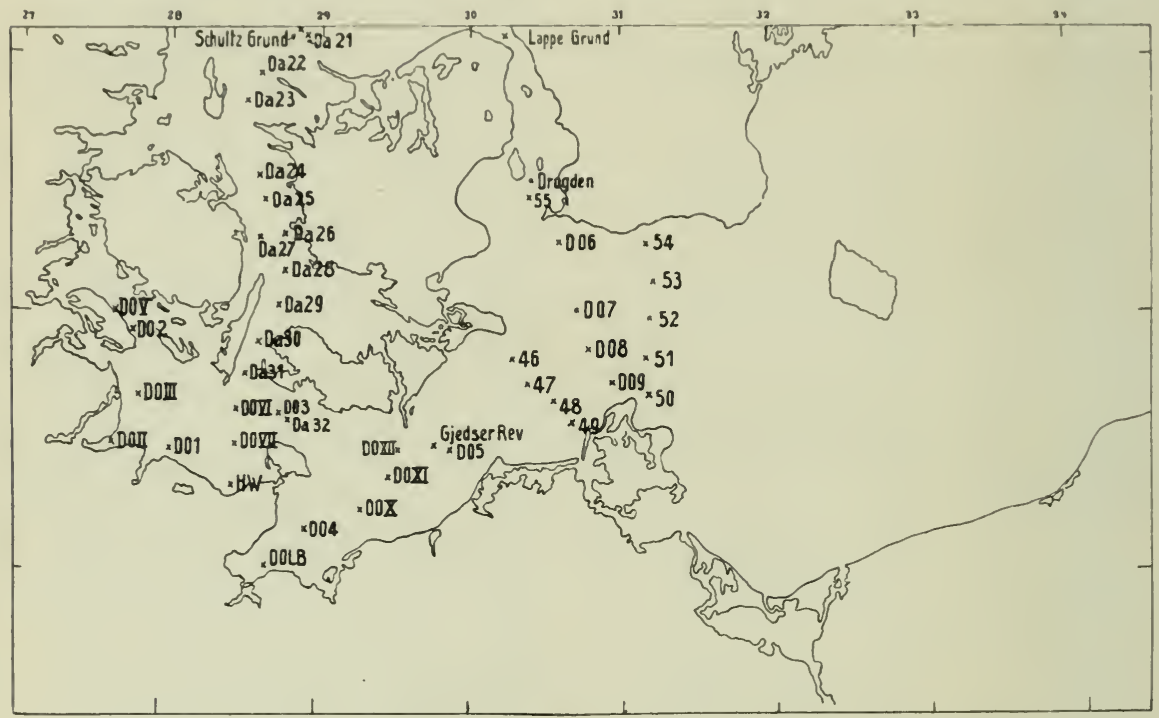

Fig. 16. Lage der Stationen für die internationalen Terminfahrten zwischen Kattegat und Gotlandsee.

(Synonyma der Stationsnummern siehe Tabelle S. 106.)

fikation in der Verteilung der Stationen und in der Wahl der Monate, liegt doch nur eine vierjährige einheitliche Periode von Beobachtungen für die dänischen und deutschen Stationen vor, die von November 1902 bis August 1906 läuft. Die großen Schwankungen, die wir auf Grund einer neunjährigen Periode kennen lernten, lehren uns, daß nur zu gleichen Zeiten gesammeltes Material miteinander verglichen werden kann, nicht aber solches aus verschiedenen Jahren oder Mittelbildungen für verschiedene Längen.

In der Regel liegen Beobachtungen von fast allen Stationen aus den Tiefen von $0 \mathrm{~m}, 5 \mathrm{~m}, 10 \mathrm{~m}, 15 \mathrm{~m}, 20 \mathrm{~m}, 30 \mathrm{~m}$ und je $10 \mathrm{~m}$ weiter 
vor. Fehlt eine Tiefe, so habe ich die Lücke durch Interpolation ergänzt. Lag sie in der Mitte, so habe ich die gleiche Progression des Salzgehaltes zwischen der nächst höheren und nächst tieferen Messung zugrunde gelegt, lag sie an der Oberfläche, so habe ich das Mittel der Differenzen zwischen $0 \mathrm{~m}$ und $5 \mathrm{~m}$ in den drei übrigen Serien aus derselben Jahreszeit in Anschlag gebracht. Die Werte für das untere Ende einer Stationsserie mußten zum weitaus größten Teile alle auf eine einheitliche Bodentiefe bei den einzelnen Stationen reduziert werden, denn die Entnahme der Salzgehaltsproben vom Boden, in der Regel einen halben Meter über dem Grunde, schwankt um einige Meter in der Tiefe, da die Lage der Stationen auf See begreiflicherweise nicht bei jeder Terminfahrt immer die gleiche ist, auch wenn dieselbe Position verzeichnet wird. Einige Male ist sie sogar absichtlich étwas verlegt worden, so bei dem Schnitt Arkona-Trelleborg mit Rücksicht auf die dort gelegten Kabel. Diese Ausnahmen von den Angaben in der Tabelle habe ich hier zusammengestellt.

D 010 5. II. 1906: $54^{0} 38^{\prime}, 14^{0} 47^{\prime} ; 4$ II. 1905 und 12. V. $1904: 15035^{\prime}$.

DO 9 31. X. 1902 bis 9. V. 1903: $54^{0} 43^{\prime}, 13^{\circ} 21^{\prime}$.

Do 8 31.X. 1902 bis 9. V. 1903: $54^{0} 55^{\prime}, 13^{0} 18^{\prime}$.

D 07 31.X. 1902 bis 9.V. 1903: $13^{0} 15^{\prime}$; 2. XI. 1904: $55^{0} 09^{\prime}, 13^{0} 05^{\prime}$.

D 06 31.X. 1902 bis 9. V. $1903: 55^{0} 16^{\prime} 13^{0} 13^{\prime}$; 2. XI. $190 \pm$ bis 2. II. 1905 : $55^{0} 13$

$12^{\circ} 58^{\prime}$

D 03 1. XI. $190 t$ bis 2. VIII. 1905̆: $54^{0} 37^{\prime}, 11^{0} 01^{\prime} ; 10$. V. $1904: 54^{0} 39,5^{\prime}, 10^{0} 57, \check{5}^{\prime}$. DO 2 29. X. 1902 bis 15. XI. 1903: $54^{\circ} 56^{\prime}, 10^{\circ} 26^{\prime}$.

Über die Lage der Stationen diene die Skizze auf S. 7) zur Orientierung, die genauen Positionen siehe S. 86-91.

Die Interpolation resp. Reduktion für das untere Ende, bei der es sich in der Regel nur um 1-2 m handelt, ist gleichfalls unter der Annahme gleicher Progression des Salzgehaltes geschehen, sei es, ob eine größere oder geringere Tiefe als die interpolierte gemessen war. In einigen Fällen ist jedoch davon abgesehen worden, indem beim Messen einer geringeren Tiefe augenfällig, wie es eine große Reihe anderer Fälle, die nicht interpoliert zu werden brauchte, dartat, nach einer ausgeprägten Sprungschicht eine Zunahme des Salzgehaltes unter der Sprungschicht nur ganz langsam erfolgte. Hätte ich die Differenzen zwischen den beiden letzten Beobachtungen in Anschlag gebracht, so wäre ein abnorm hoher, nicht der Wirklichkeit entsprechender Salzgehalt in die Rechnung eingeführt worden.

Die Mittel habe ich in den Tabellen S. 86-91 zusammengestellt. Das Datum gibt die Extreme der Bcobachtungstermine wieder. Bei einigen 
Stationen lagen Doppelserien mit nur wenigen Tagen Untersehied vor. Zu diesen sei bemerkt, dab ich die Serie rom 14. Angust 1904 bo 6 und rom 12. August 1901 für DO 7 fortgelassen habe, bei DO 8 jene rom 9. September 1904 und bei Da 23 jene vom 2.. Mai und 2. November, soweit sie Doppelserien waren, die zweite Messung rom 2. August 190:3 und 1. August 1904 und rom t. Februat 1903, 3. Februar 1904, 1. Februar 1905 und vom ㄹ. Februar 1906. Maßgebend war für die Auswahl eine günstige Zeitlage und Tiefenentnahme, so daß ein Interpolieren möglichst vermieden werden konnte.

Auf den deutschen Fahrten der Internationalen Meeresforschung brauchte man Richtersche Tiefseethennometer, die anfangs in der physikalisch-technischen Reichsanstalt in Charlottenburg geprüft wurden, später den internationalen Abmachungen entsprechend im Zentrallaboratorium in Kristiania. Sie waren teils in Fünftel, teils in Zehntel Grade geteilt. Als Wasserschöpfer diente bei den essten Fahrten der ron Pettersson-Nansen, dann der von Krümmel konstruierte. Neben Aräometerbestimmungen des Salzgehaltes, die gleich an Bord ausgeführt wurden mit geprüften Instrumenten in ähnlicher Weise wie auf den dänischen Feuerschiffen und mit Hilfe ron Knudsens Tabellen umgerechnet wurden, sind sämtliche Proben im Kieler Laboratorium gemäß den internationalen Bestimmungen und unter Benutzung von Normalwasser titriert worden. In der gleichen Art wurde das auf den dänischen und schwedischen Fahrten erzielte Material in Kopenhagen und Stockholm rerarbeitet. Es wurde fast auf dieselbe Weise cewonnen, nur in den benutzten Wasserschöpfern bestehen Abweichungen, indem man bald zu dem ron Pettersson-Nansen, bald zu dem von Knudsen oder Krümmel oder anderen verwandten Konstruktionen griff. $\left.{ }^{1}\right)$ Die Genauigkeit soll nach den internationalen Kongreßbeschlüssen zu Stockholm und Kristiana bis $0,05^{\circ}{ }_{00}$ gehen, in Wirklichkeit ist nach den praktischen Erfahrungen die Fehlergrenze etwa zwischen 0,02 und $0,03 \%$ Mille gelegen.

Trotz der großen Genauigkeiten sowohl hinsichtlich der Beobachtungsmethoden wie der Sorgfältigkeit in der Gewinnung der Messungen können die Ergebnisse doch nur als angenäherte Werte gegenüber den Naturverhältnissen betrachtet werden. Denn nicht nur die Zeit der Termine schwankt, sondern auch die Wasserzusammensetzung schwankt an den Terminen, und zwar teilweise recht erheblich, wie die Dis-

1) Nach den einzelnen Berichten über: Die Beteiligung Deutschlands an der internationalen ILeeresforschung und nach der Einleitung zu den einzelnen Jahrgīngen des Bulletin trimestriel. 
kussion an den Beobachtungen an den vier Feuerschiffen gezeigt haben dürfte. So kommt es auch, daß einander so dicht gelegene Stationen wie $\mathrm{Da} 32$ und DO 3 , beide in Fehmarnbelt in unmittelbarer Nachbarschaft, recht erhebliche Differenzen voneinander geben, und zwar ganz regelloser Art, wie die folgende kleine Zusammenstellung dartun möge, die die gegenseitigen Differenzen mit einer positiven Abweichung bringt, wenn bei DO 3 der Salzgehalt höher als bei Da 32 war, negativ im umgekehrten Sinne. Der Winter muß fortfallen, da wir über ihn bei Da 32 wegen fehlender Beobachtungen kein Mittel bilden können.

\begin{tabular}{c||c|c|c}
\multicolumn{2}{c}{ Salzgehaltsdifferenz } & D $03-D$ a 32 in ${ }^{0} 0^{\circ}$ \\
\hline Tiefe & Frühjahr & Sommer & Herbst \\
\hline \hline $0 \mathrm{~m}$ & $+0,92$ & $+0,32$ & $-0,79$ \\
$5 \mathrm{~m}$ & $+1,14$ & $-0,52$ & $-1,01$ \\
$10 \mathrm{~m}$ & $+0,17$ & $+2,35$ & $-1,30$ \\
$15 \mathrm{~m}$ & $-1,05$ & $-0,18$ & $-0,49$ \\
$20 \mathrm{~m}$ & $-0,72$ & $+1,24$ & $-0,55$ \\
$30 \mathrm{~m}$ & $-0,41$ & $+1,58$ & $+0,27$
\end{tabular}

Wir sehen Unterschiede in dem beträchtlichen Ausmaß von 2,35\% und lernen daraus, daß wir mit dem Ziehen von Schlußfolgerungen uns auf die größeren Differenzen beschränken müssen. Denn es kommt. noch hinzu, daß die Terminfahrten weder als Extreme noch als Mittel der Jahreszeiten angesehen werden können, wie aus einem Vergleich mit dem Gange der Beobachtungen an den Feuerschiffen sofort erhellt.

Aus den Berechnungen zeigt sich deutliche die Zunahme des Salzgehaltes von der Arkonasee zum Kattegat. Ein Salzgehalt an der Obcrfläche von $7,52 \%$ im Winter am Westende der Gotlandsee hebt sich auf $24,72 \%$ im südlichen Kattegat. Da die Oberflächenschwankungen in diesem größer sind als in der Gotlandsee, so sind die jahreszeitlichen Differenzen naturgemäß verschiedene.

Legen wir die Mittel des Schnittes Trelleborg-Arkona zugrunde, so betragen die Abweichungen die umstehenden Werte.

Wir sehen, daß das Steigen nicht immer wahrzunehmen ist. Im Winter haben Da 29, Da 26 und ganz beträchtlich Da 23 und 22 zusammen geringeren Salzgehalt als ihre südliche Nachbarstation. Im Frühjahr hat der Fehmarnbelt sowohl auf der deutschen wie auf der dänischen Station einen auffallend hohen Salzgehalt, die gleiche Abweichung weist im kleineren Maßstabe Da 27 auf. Im Sommer fällt wieder der Fehmarnbelt auf, und von Da 30-28 im südlichen Großen Belt nimmt der Salzgehalt nordwärts sogar während dreier Stationen ab. 
Abwoichungen der Salmgehaltsmittel vom Schuitt Arkona-Trelleborg für die oberflíche in ${ }^{0}{ }_{00}$.

\begin{tabular}{|c|c|c|c|c|c|}
\hline Station & Winter & I'rülụialır & Sommer & Hollost & Jahr \\
\hline DO 5 & 4,13 & $0,8.5$ & 0,72 & 2,44 & 1,54 \\
\hline I) a :32 & - & 4,66 & 5,05 & 5,61 & - \\
\hline 1003 & 8,60 & 5,58 & 5,27 & 4,83 & 6,07 \\
\hline $\mathrm{DO} 4$ & $7,0: 3$ & $3,2 \cdot 2$ & 3,16 & $4, \times 6$ & 4,82 \\
\hline 1) 01 & 9,38 & 7,50 & 5,81 & 7,48 & 7,033 \\
\hline DO 2 & $10,7+$ & 7,82 & 6,89 & 8,17 & 8,41 \\
\hline 1) a 31 & 11,23 & 4,15 & 4,37 & 6,61 & 6,59 \\
\hline 1) a 30 & 11,60 & 4,61 & 4,56 & 6,69 & 6,87 \\
\hline D a 29 & 11,29 & 5,23 & 4,53 & 7,02 & 5,02 \\
\hline 1) a 28 & 11,79 & 5,30 & 4,34 & 7,45 & 7,22 \\
\hline D) a 27 & 12,50 & 7,10 & 5,48 & 7,87 & 8,24 \\
\hline D a 26 & 10,87 & 5,23 & 4,89 & 7,32 & 7,33 \\
\hline ป) a 25 & 13,25 & 6,93 & 6,12 & 8,29 & 8,63 \\
\hline 1) a 24 & 13,57 & 7,91 & - & 9,39 & - \\
\hline 1) a 23 & 12,53 & 7,81 & 5,91 & 9,26 & 8,88 \\
\hline D a 22 & 13,23 & 8,43 & 7,08 & 9,87 & 9,64 \\
\hline D a 21 & 14,05 & 9,83 & 7,52 & 10,66 & 10,52 \\
\hline Da 20 & 14,50 & 9,98 & 8,42 & 11,59 & 11,13 \\
\hline Da 18 & 16,27 & 12,60 & 11,38 & 12,49 & 13,18 \\
\hline
\end{tabular}

Zeit und Mächtigkeit der Salzgehaltsextreme.

\begin{tabular}{|c|c|c|c|c|c|c|c|c|}
\hline \multirow{3}{*}{ Station } & \multicolumn{4}{|c|}{ Oberfläche } & \multicolumn{4}{|c|}{ Boden } \\
\hline & \multicolumn{2}{|c|}{ Maximum } & \multicolumn{2}{|c|}{ Minimum } & \multicolumn{2}{|c|}{ Maximum } & \multicolumn{2}{|c|}{ Minimum } \\
\hline & $\begin{array}{c}\text { Jahres- } \\
\text { zeit }\end{array}$ & $\mathrm{m}$ & $\begin{array}{c}\text { Jahres- } \\
\text { zeit }\end{array}$ & $\mathrm{m}$ & $\begin{array}{c}\text { Jahres- } \\
\text { zeit }\end{array}$ & $\mathrm{m}$ & $\begin{array}{c}\text { Jahres- } \\
\text { zeit }\end{array}$ & $\mathrm{m}$ \\
\hline Da 18 & W & $0-10$ & $\mathrm{~S}$ & $0--10$ & $\mathrm{~F}$ & $20-45$ & W S & $0-45$ \\
\hline I) a 20 & IV & $0--15$ & $\mathrm{~S}$ & $0-10$ & $\mathrm{H}$ & 27 & W S & 27 \\
\hline Da 21 & IV & $0--10$ & $\mathrm{~S}$ & $0-10$ & $\mathrm{H}$ & $30-36$ & W S & $30-36$ \\
\hline Da 22 & W & $0-10$ & $\mathrm{~S}$ & $0--10$ & $\mathrm{H}$ & $15-50$ & IV & $15-50$ \\
\hline Da 23 & W & $0-10$ & S & $0-5$ & $\mathrm{H}$ & $30-50$ & W & $20-50$ \\
\hline D a 25 & W & $0-15$ & $\mathrm{~S}$ & $0-1 \check{0}$ & $\mathrm{H}$ & 30 & W & $20-30$ \\
\hline Da 26 & W & $0-10$ & $\mathrm{~S}$ & $0-10$ & $\mathrm{~S}$ & $20-55$ & W & $20-555$ \\
\hline Da 27 & W & $0-10$ & $\mathrm{~S}$ & $0-5$ & $\mathrm{~S}$ & $20-25$ & IV & $20-25$ \\
\hline Da 28 & WV & $0-15$ & $\mathrm{~S}$ & $0-10$ & $\mathrm{~S}$ & $20-40$ & IV & $20-40$ \\
\hline Da 29 & IV & $0-15$ & $\mathrm{~S}$ & $0-10$ & $\mathrm{~S}$ & $20--30$ & IV & $20-30$ \\
\hline D a 30 & IV & $0-15$ & F & 0 & $\mathrm{~S}$ & $20-50$ & W & $20-50$ \\
\hline D a 31 & IV & $0-15$ & $\mathrm{~F}$ & 0 & $\mathrm{~S}$ & $20-31$ & W & $20-30$ \\
\hline DO2 2 & $\mathrm{IT}$ & $0-15$ & $\mathrm{~S}$ & $0-5$ & $\mathrm{~S}$ & $20-33$ & W & $30-33$ \\
\hline D 01 & W & $0-15$ & $\mathrm{~S}$ & $0-\overline{5}$ & W S & 19 & $\mathrm{~F} \mathrm{H}$ & $10--19$ \\
\hline DO 4 & IV & $0-15$ & $\mathrm{~S}$ & 0 & S & $20-22$ & IV & 22 \\
\hline DO 3 & WT & $0-15$ & $\mathrm{H}$ & $0-5$ & W S & $20-33$ & F H & $30-33$ \\
\hline D0 כ & IV & $0-15$ & $\mathrm{~S}$ & 0 & W S & $20-26$ & F H & $20-26$ \\
\hline D 06 & IV S & $0-29$ & $\mathrm{~F} \mathrm{H}$ & $0-29$ & W S & $0-29$ & F H & $0-29$ \\
\hline DO 7 & IV & $0-20$ & $\mathrm{H}$ & $0-20$ & W S & $30-37$ & $\mathrm{~F} \mathrm{H}$ & $30-37$ \\
\hline DO 8 & IV & $0-20$ & $\mathrm{~F}$ & $0-20$ & IV S & $30--44$ & $\mathrm{FH}$ & $30-44$ \\
\hline D 09 & IV S & $0-20$ & F H & $0-5$ & IV S & $30-34$ & F H & $3 t$ \\
\hline
\end{tabular}


Da 26 zeichnet sich wieder durch geringere Werte aus, ebenso das Südende des Samsöbeltes. Auch im Herbst fällt Da 26 aus der Reihe heraus und Da 23 bleibt um ein geringes gegen Da 24 zurück. Eine kleine Unregelmäßigkeit zeigt dann noch Da 19 im Kattegat.

Im Jahresmittel steht $\mathrm{Da} 26$ zu niedrig. Die Ursache dürfte die ausgesprochene östliche Lage im Großen Belt sein, indem der süßere Auslauf nach dieser Seite gedrängt wird. Das umgekehrte scheint für die Stationen des Fehmarnbelt zu gelten, wenigstens reicht mutmaßlich im Frühjahr und Sommer das Einlaufwasser an der Oberfläche bis zu den Stationen. Daß bei der sommerlichen Abnahme von Da 30-28 eine Beeinflussung durch Smaalands Fahrwasser vorliegt, indem von diesem sich eine Decke süßen Wassers ausbreitet, ist wahrscheinlich.

Am Boden finden wir auf dem Wege von dem Kattegat zur Ostsee im Winter eine Ausnahme in der Abnahme bei Da 26, Da $\leftleftarrows 8$, DO 3, im Frühjahr bei $\mathrm{Da} 25$ und $\mathrm{Da} 28$, im Sommer ist keine vorhanden, im Herbst bei Da 23. Die Abweichungen am Boden sind also geringer als an der Oberfläche. Da 28 zeichnet sich durch hohen Salzgehalt aus, was auch im Jahresmittel zutage tritt. Eine Begründung vermag ich. weder aus der Position noch aus der Tiefe zu geben. Die Zunahme bei $\mathrm{Da} 26$ im Winter dürfte den Hinweis bieten, daB in dieser Jahreszeit das Bodenwasser östlich Sprogö läuft.

Auch für die Abweichungen haben wir versucht, die Mächtigkeit zu ermitteln. In $30 \mathrm{~m}$ zeigt eine aus der Reihe fallende Zunahme im Winter Da 31 und DO 3, im Frühjahr Da 25, Da 28, Da 31, im Sommer Da 23, Da 25, Da 29, Da 31, im Herbst Da 24, im Jahresmittel Da 31; für $20 \mathrm{~m} \mathrm{Da} \mathrm{20,} \mathrm{für} 15 \mathrm{~m} \mathrm{Da} \mathrm{22} \mathrm{und} \mathrm{Da} \mathrm{21,} \mathrm{Da} \mathrm{27,}$ $\mathrm{Da} 30$, Do 3, für $10 \mathrm{~m} \mathrm{Da} 22$, Da 27, Da 30, für $5 \mathrm{~m} \mathrm{Da} 27$ und Da 31. Da 20 in den tieferen Schichten muß seine Ursache in Zirkulationen im Kattegat haben, Da 27 in einer Trennung des Großen Beltes bei Sprogö in eine westliche und östliche Rinne, bei Da 30 führt Smaalands Fahrwasser mutmaßlich eine geringe Herabsetzung herbei.

Wir haben die jahreszeitlichen Veränderungen vom südlichen Kattegat an durch den Großen und Stammbelt bis in die Arkonasee in Schnitten dargestellt. Bei der Mittelbildung aus nur 4 Jahren ist es selbstverständlich, daß damit nur ein ungefähres Bild gewonnen werden kann und daß bei den großen Veränderungen, die der Salzgehalt zu jeder Jahreszeit erfährt, auch die Werte unter sich nicht entsprechen können. Kommt es doch mehrfach vor, daß selbst in der Mittelbildung der Salzgehalt nicht regelmäßig nach der Tiefe zunimmt, sondern sich 
II. Spethmann.

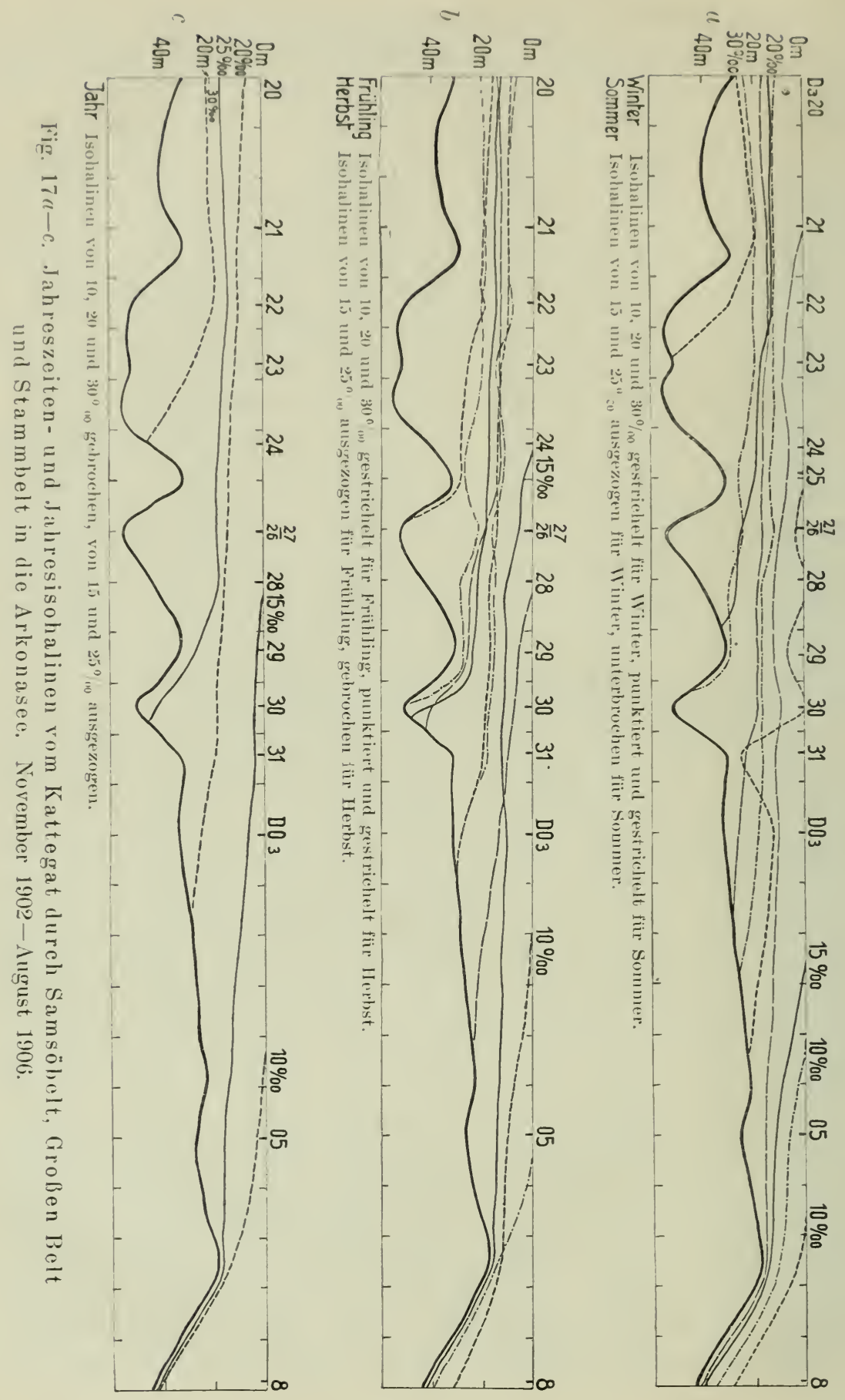


etwas vermindert. Näheres Studium zeigt, daß hierbei nicht eine genetische Ursache mitspielt, sondern daß die Abweichungen auf Beobachtungsungleichheiten zurückgehen.

Wir sehen sofort, daß das $30^{\circ} \%$ Wasser im Sommer und Herbst am weitesten einwärts dringt, bis fast zum Südende des Großen Beltes. Einmal wurde es sogar in über 10 m Mächtigkeit in Da 30 gefunden, am 1. August 1905, während es in Winter nie über $\mathrm{Da} 28$ hinausgegangen ist. Wie große Ausnahmen es aber geben kann, zeigt eine Fahrt von Ende Mai 1909. Man fand am Boden der deutschen Station des Fehmarnbeltes $30,81 \%$ ! Nicht so großen Schwankungen ist das Ausbreitungsgebiet von $25 \%$ unterworfen, das meistens nicht über den Großen Belt hinausgeht. Nur im Sommer treffen wir es bei der Mittelbildung auch in DO 3 an, in der Kadetrinne ist es in den Terminen niemals bemerkt worden. Der höchste Salzgehalt, den man hier auf allen Terminfahrten zusammengenommen beobachtete, betrug $24,94 \%$ am 2j. Mai 1909. Darauf fulgt $24,60^{\circ}$ \% von $23-24 \%$ wurde des öfteren konstatiert.

Entgegengesetzt zu den Bodenschichten greift das süßere Wasser im Frühjahr am weitesten aus der Arkonasee heraus. Im Winter geht in der Mittelbildung die $10^{\circ}$ on Isohaline nur bis zur Darsser Schwelle, im Frühjahr bis zum Anfang des Fehmarnbeltes. Daß sie sich gelegentlich bis Schultz Grund erstrecken kann, erwähnten wir schon. Die $15 \%$ Isohaline reicht im Sommermittel sogar bis durch den Samsöbelt, während sie im Winter nicht aus dem Stammbelt herausgeht. Sie drängt auch die $20^{\circ} \%_{00}$ Isohaline etwas zurück, wenngleich bei dieser noch eine andere unbekannte Ursache im Zurückweichen mitzuwirken scheint, indem sie im Frühjahr und Herbst am weitesten nordwärts geht.

Würdigen wir das Gesamtbild der Schnitte, so zejgen sie, wie sich die Gegensätze im Winter auf den ganzen Längsschnitt verteilen, in den drei übrigen Jahreszeiten sich dagegen erst vom südlichen Großen Belt an einwärts einstellen. Im Jahresmittel kommt diese Wanderung natürlich nicht zum Ausdruck, es zeigt, wie die Isohalinen, je weiter landeinwärts, umso stärker gekrümmt sind.

Betrachtet man die Veränderungen ron Jahreszeit zu Jahreszeit, so nimmt man, wie auf Grund der Ergebnisse an den dänischen Feuerschiffen zu erwarten war, deutlich Schwankungen an ihnen wahr. Am leichtesten müssen sie in der Tiefe zu überblicken sein, die den wenigsten Einflüssen ausgesetzt ist. Um sie zu verstehen, wollen wir nicht einen Horizontalschnitt durch ein Niveau legen, sondern wählen die jeweiligen Bodenangaben. 
I 11 les

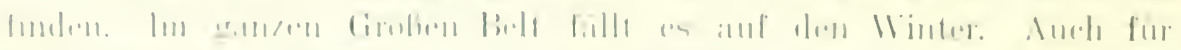

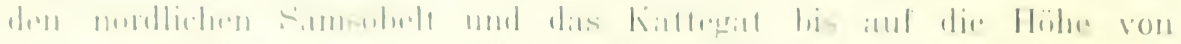

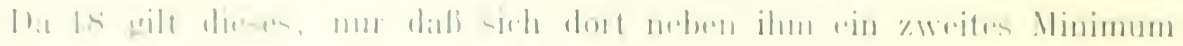

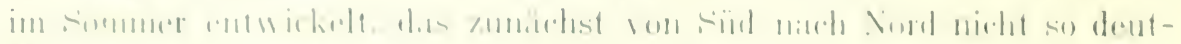

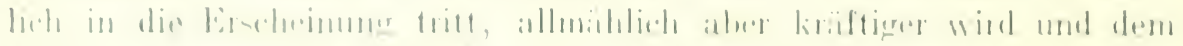

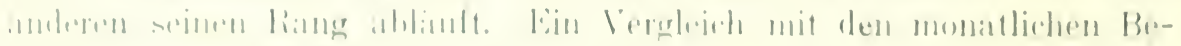

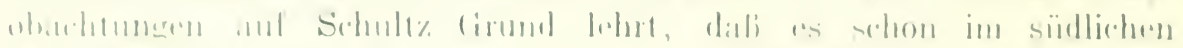

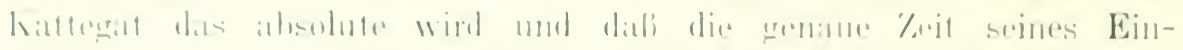

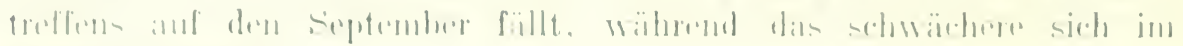

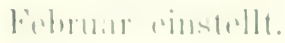

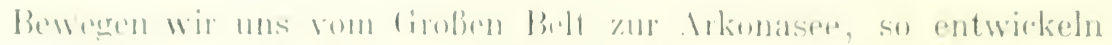

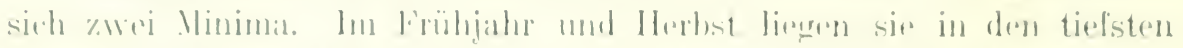

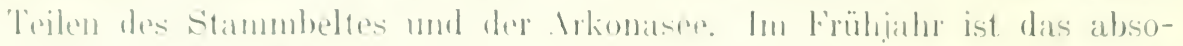

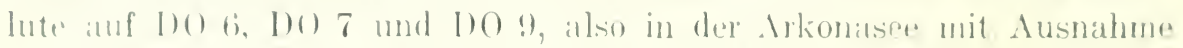
rom Do \&. Dieses besitzt das absolute Minimmon im Herbst, das grledehe Bild gilt für den Boden der Kadetrime und der Kieler Bucht, während sich der Alsenbelt dem Groben Belt anschließt. J)ie Verhältnisse am Boden vom Fehmambelt sind ungeklärt, die gregenseitigen Differenzen gering und auf der deutschen umb dinischen Station einander widersprechend.

Ln Klarheit über das Auftreten der Minima im Stammbelt und in der. Irkonasee zu gewinnen. habe ich die Beobachtungen auf Fehmarnbelt-Fenerschiff herangezogen, das auf 2n m Tiefe gelegen ist und Aufschlub über die salzgehaltsschrankungen in den größeren Tiefen gibt. Jie - nicht veröfentlichten - Messungen von 1903 bis 1911 sind bereits von Ruppin in Dekadenmitteln rerechnet. die wir in Monatsmittel neu umgewertet haben. Die Messungen finden zwar am Morgen und Abend statt, standen aber nicht unter so scharfer Kontrolle wie jene ron den vier besprochenen Feuerschiffen. Außerdem sind größere Lüchen in den Reihen vorhanden, so daß wir das Feuerschiff bis jetzt unberüchichtigt gelassen haben. In der Mittelbildung für neun Jahre dürften wir aber doch ein ungefähres Bild über den (iang des Salzgehaltes gewinnen. trotzdem, wie Ruppin sagt, die einzelnen Beobachtungen mit erheblichen Fehlern belastet sein mögen und besonders hin und wieder offensichtliche schreibfehler vorkommen. Am Feuerschiff zeigen sich gleichfalls die beiden Ninima im April und Oktober, Am Schultz Grund fielen sie aul Februar und September, also ein bis zwei Monate frïher. Da die Maxima auf Schultz Grund auch um den gleichen Betrag früher gelegen sind als auf Fehmarnbelt (Schultz Grund 
Juni und November, Fehmarnbelt Juli und Januar), so ist der Schluß nahe, daß die zeitliche Differenz durch den Weg bedingt ist, den das einlaufende Wasser zurückzulegen hat, um von dem einen Feuerschiff zu dem andern zu gelangen. Für die Resultate auf den internationalen Terminfahrten ergibt sich demnach, daß das Frühjahrsminimum des Stammbeltes und der Arkonasee das fortgepflanzte Winterminimum des Großen Beltes ist.

Sofort drängt sich der Gedanke auf: Da auch das herbstliche Minimum sich von Schultz Grund-Feuerschiff bis Fehmarnbelt um einen Monat verzögert, wie kommt es, daß es durch die Terminbeobachtungen im Großen Belt nicht zum Ausdruck gebracht ist. Da es ziemlich genau in die Mitte zwischen zwei Terminbeobachtungen fällt, so kann es sich der Wierlergabe entzogen haben. Würden wir beispielsweise auf Schultz Grund nur im Maï und August beobachten, so würde uns ein zwischen ihnen gelegenes Maximum verloren gehen. Auch bei dem Maximum des Winters drängt sich die Frage auf, wo es in den Terminbeobachtungen des Großen Beltes bleibt. Auf Da 25 nördlich Sprogö ist es noch wahrzunehmen. Mutmaßlich steht ein anderer Grund dahinter als gerade das Fallen zwischen Terminbeobachtungen, vielleicht spielt der Stromeinfluß in der talartigen Zusammenfassung des Wassers in der Rinne des Großen Beltes eine Rolle.

An Maximis stellen sich in der Arkonasee und im Stammbelt zwei ein, eins im Winter und eins im Sommer, von denen bald das eine, bald das andere das absolute ist. Im Schnitt Rügen-Schonen übertrifft mit Ausnahme im deutschen Küstengebiet das winterliche Maximum. Der Salzgehalt am Boden bei Arkona geht mit dem am Boden der Kadetrinne und des Fehmarnbeltes parallel und weist auf eine genetische Zusammengehörigkeit von einströmendem Wasser hin. Denn diese Ausnahmen werden im Großen und Kleinen Belt die Regel, wo ein Salzgehaltsmaximum am Boden im Sommer existiert, das sich in das Kattegat hinein erstreckt und dort nach den Terminbeobachtungen scheinbar auf den Herbst übergeht, gleichzeitig entwickelt sich hier aber auch ein Maximum im Frühjahr.

Ob das Sommermaximum sich aus dem Frühjahrsmaximum des Kattegat entwickelt, vermögen wir erst durch Vergleich mit den Ergebnissen auf Schultz Grund zu entscheiden, von dem wir schon für das Tiefenwasser die beiden Maxima kennen lernten. Das im Frühjahr ist das größere, so daß wir unser Bild vervollständigen können. Das in die Beltsee einlaufende Frühjahrsmaximum (Juni auf Schultz Grund) macht sich in der Arkonasee erst im Sommer bemerkbar. 
Wir haben ein Durchwandern der Extreme des Salygehaltes durch die Beltsee kennen gelemt. Um die Ursache zu ergründen, wollen wir zunächst prüfen, ob ein Zusammenhang zwischen Oberflächenextremen und Bodenextremen besteht, derart, daB auf Reaktionswirkungen geschlossen werden kann. Betrachten wir, ob Minima an der Oberfläche mit Maximis am Boden zusammenfallen. Wir stellen es gar nicht fest in der Arkonasee, dagegen im Stammbelt bis zum Alsenbelt, im Großen Belt von Da 29 bis Da 25, gar nicht im Samsöbelt und in dem südlichen Kattegat. Umgekehrt fallen Ninima am Boden zusammen mit Maximis an der Oberfläche in der Lübecker Bucht, im Alsenbelt und im Großen Belt bis zum Beginn des Kattegat. Die Verteilung ist also recht unregelmäßig und würdo das noch mehr sein, wenn monatliche Beobachtungen rorlägen, wie für die dänischen Feuerschiffe. Bei diesen korrespondieren die Kurven für den Salzgehalt am Boden mit denen für die Oberfläche weder bei Lappe Grund noch bei Schultz Grund. Versuchen wir deshalb außerhalb der Beltsee Anhaltspunkte für die Herkunft des Salzgehaltes zu erhalten.

Zu dem Ende habe ich zunächst die Bodenschwankungen auf den Feuerschiffen Anholt Knob und Skagens Rev herangezogen, ' für die Ruppin die Mittel für 1903 bis 1908 schon gebildet hat. Wir dürfen sie mit unserer Periode 1903 bis 1911 in Verbindung setzen, wie ein Vergleich der Ruppinschen Zahlenwerte für Schultz Grund mit den meinen lehrt. Für die Minima ergibt sich, daß das absolute für Schultz Grund im September einen Monat früher auf Anholt Knob und Skagens Rev eintritt, dagegen das kleine des Februar nicht. Bei den Maximis kommt das größere des Juni auf Anholt Knob im Mai und Juni durch gleiche Werte zum Ausdruck, auf Skagens Rev aber ganz deutlich im Mai. Das kleinere des November verschiebt sich dagegen ebenfalls nicht.

Nähern wir uns noch mehr dem Weltmeere, so kommen wir auf die Station Skag 8, jener Station, die Pettersson für seine Golfstromschwankungen herangezogen hat. Wir haben aus den Terminbeobachtungen, die für sie vorliegen, die Höhenlage von $25^{\circ}{ }_{00}$ interpoliert und ein ausgesprochenes Minimum für Mai gefunden, also mit einer Verfrühung von zwei bis drei Monaten, dagegen Skagens Maximum rom November gleichfalls im November. Hier liegt vielleicht ein Kausalzusammenhang zwischen den Schwankungen des Atlantischen Wassers mit den Schwankungen des Salzgehaltes am Boden ron Skagens Rev vor. Leider gestatten die Einzelbeobachtungen auf Skag 8 nicht, ihn weiter zu verfolgen, da vier Beobachtungen im Jahre zu wenig sind 
und wir nicht wissen, ob nicht noch andere Schwankungen vorliegen. Vorläufig scheint der Zeitunterschied zwischen Maximis und Minimis verschieden groß zu sein.

Stellen wir nun die Ergebnisse tabellarisch zusammen, um einen Überblick über die Fortpflanzung der Salzgehaltsextreme zu gewinnen.

\begin{tabular}{|c|c|c|c|c|c|c|}
\hline \multirow[b]{2}{*}{ Mitte des Skagerrak } & \multirow{2}{*}{\multicolumn{2}{|c|}{. }} & \multicolumn{2}{|c|}{ II a x im a } & \multicolumn{2}{|c|}{ M in im a } \\
\hline & & & November & - & Mai & - \\
\hline Skagens Rev . . . & . & . & November & Mai & August & Februar \\
\hline Schultz Grund & . & . & November & Juni & September & Februar \\
\hline Fehmarnbelt . & . & . & Dezember & Juli & Oktober & April \\
\hline Arkonasee . & . & . & Februar & August & November & Mai \\
\hline
\end{tabular}

Wenn wir die Ergebnisse dieser Reihen mustern, so ist zu bedenken, daß für die Arkonasee nur die Terminbeobachtungen vorlagen, für die übrigen Stationen aber Nonatsmittel. Betrachten wir das generelle Bild, so erkennen wir ein deutliches Einwärtswandern der Extreme. Das große Novembermaximum im Skagerrak macht sich in dem gleichen Monat bis Schultz Grund bemerkbar, einen Monat später auf Fehmarnbelt und wahrscheinlich in der ersten Januarhälfte in der Arkonasee, was nicht schärfer zu erkennen ist, da die Terminfahrten einen Monat später fallen. Ich habe versucht, hier Klarheit auf Grund von Fahrten außerhalb der vier Termine zu schaffen, wie sie seit 1907 ausgeführt sind. Doch waren in ihnen keine Anhaltspunkte für die Arkonasee enthalten, während sie für die Beltsee das Ausgeführte bestätigen.

Langsamer bewegt sich augenscheinlich das Minimum des Skagerrak. Nach den vorliegenden Beobachtungen geurteilt, braucht es ein halbes Jahr bis zur Arkonasee. Daß ein Ninimum sich langsamer fortbervegt als ein Maximum, ist verständlich, daß es diesem gegenüber aber gerade auf der Strecke von der Mitte des Skagerrak bis nach Skagens Rev eine Verzögerung von drei Monaten erleidet, ist auffallend. Setzt man das Maiminimum des Skagerrak einen Monat später an, da die Terminbeobachtungen ja nicht mit dem absoluten Extrem zusammenfallen könnten, so ist die Verspätung von zwei Monaten immerhin noch bemerkenswert. Einstweilen vermögen wir ein retardierendes Moment nicht aufzudecken.

Von Skagens Rev an können wir in ähnlicher Weise ein zweites Maximum und Minimum verfolgen, für das wir bei den wenigen Beobachtungen in Skag 8 keine Anzeichen finden. Seine zeitliche Terspätung auf dem Wege zur Gotlandssee weist aber auch auf eine Herkunft aus dem Skagerrak hin. 
Wir sind somit zu dem Ergebnis gelangt, daß die Schwankungen der Extreme in der Arkonaseo dio Ausläufer von Schwinkungen des Atlantischen Wassers an der südnorwegischen Küste verkörpem. Die große Herbstanschwellung des Atlantischen Wassers macht sich im Winter in der Arkonasee hemerkbar.

Auf der Oberfläche wird auf allen Stationen das salzreichste Wasser im Winter angetroffen. Das Minimum ist nicht so einheitlich entwickelt. Im Kattegat und Samsöbelt fällt es deutlich auf den Sommer, und einheitlich gilt das gleiche fast für den ganzen Großen Belt, nur daß3 die Differenz gegenüber dem Frühjahr abzunehmen scheint. Am Südausgang des Großen Beltes versehiebt sich das Oberflächenminimum auf diese Jahreszeit. Wir finden das gleiche auf der dänischen Station des Fehmainbelt und in der Arkonasee mit Ausnahme von DO 7; um $0,6^{\circ} \%$ ist im Sommer der Salzgehalt geringer bei der Kadetrinne, um $0,24 \%$ auf DO 3, um $0,01^{\circ}{ }_{00}$ in der Lübecker Bucht. Soweit man diesen geıingen Werten Gewicht beilegen darf, scheint sich in diesem Gebiet der Ubergang eines Frühjahrminimums gegen das eines Sommerminimums zu vollziehen oder sich wenigstens etwas zu verspäten. Hierfür spricht anch das um einen Monat spätere Auftreten des Minimums bei Schultz Grund, das bei Lappe Grund wegen der bedeutend kürzeren Entfernung zwischen Arkonasee und Kattegat nicht wahrzunehmen ist. Die Ursache der Verzögerung liegt in der Zeit, die das Zurücklegen des Weges für das Süßwasser erfordert.

Frïhjahr und Herbst bieten natürlich Übergänge zwischen den Extremen. Der Salzgehalt des Herbstes ist überall größer als der des Frühlings mit Ausnahme einiger weniger Stationen. Diese sind DO 3, wieder im Gegensatz zu dem benachbarten Da 32, und DO 7. Bei dem letzteren fehlen $0,02{ }^{\circ} / 00$ im Herbst, um das Frühjahr zu erreichen, so daß hier kein besonderer Wert auf das Ergebnis zu legen ist. Für DO 3 geht der auffällige Mangel von $0,64 \%$ vielleicht darauf zurück, daß es zeitweise in Auslaufwasser des Fehmarnbeltes gelegen ist, das sich in der Regel auf die Nordseite beschränkt, wie wir noch kennen lernen werden. Das Maximum liegt auf Fehmarnbelt im Januar, auf Schultz Grund im Februar, woraus erhellt, daß es deutlicher als das Minimum eine Verzögerung auswärts erleidet. Es tritt auf Fehmarnbelt und auf Schultz Grund im Juni ein.

In $5 \mathrm{~m}$ Tiefe lassen sich die Schwankungen noch schärfer überblicken. Ton Da $30 \mathrm{im}$ südlichen Großen Belt nordwärts ist ein höchster Salzgehalt im Sommer beobachtet worden, nur Da 20 fällt aus dem Rahmen heraus. Von da an westwärts ist das Minimum im Frühjahr mit 
Ausnahme wieder bei DO 7. Da diese Station beide Male von dem allgemeinen Bilde abweicht, scheint keine zufällige Ursache obzuwalten. Die Station liegt ziemlich in der Mitte zivischen Arkona und Trelleborg, so daß vielleicht zur Erklärung heranzuziehen ist, daß zu ihm die Süßwasserzufuhr seitens des Landes mit einer Verspätung gelangt.

Für die Schwankungen an der Oberfläche lernen wir also ein Herauswandern aus der Gotlandsee kennen. Im Kattegat finden wir das Minimum im Juni, auf Anholt Knob und Skagens Rev im gleichen Monat, scheinbar ohne Verzögerung, doch dürfen wir nicht aus den Augen lassen, daß der Sund ein wichtiges Austrittstor für das süßere Oberflächenwasser ist. So kommt es, daß wir bei Lappe Grund dem Minimum schon im Mai begegnen.

Auf Grund der gewonnenen Erfahrung wollen wir zu einer Analyse der Schwankung zwischen der Oberfläche und dem Boden schreiten Wir haben auf der Tabelle S. 76 die Niveaus festgestellt, bis zu denen sich die Extreme der Oberfläche und des Bodens geltend machen, wobei auch darauf geachtet wurde, daß das gegenseitige Verhältnis von zwei Extremen das gleiche blieb. Die Tiefenzahlen geben ein überraschend klạres Bild. Die Schwankungen der Bodenmaxima machen sich nur bis $20 \mathrm{~m}$ hinauf bemerkbar, vom nördlichen Großen Belt an nordwärts mit Ausnahme von Da 22 und in der Arkonasee nur bis $30 \mathrm{~m}$. Die Station DO 6 macht eine Ausnahme, die darauf zurückgeht, daß die Extreme an der Oberfläche und am Boden zu gleichen Zeiten eintreten.

Die Maxima der Oberfläche lassen sich bis zu 15 m Tiefe dort verfolgen, wo die Bodenmaxima bis zu $20 \mathrm{~m}$ heraufreichen, wovon der nördliche Große Belt bei Sprogö eine Ausnahme macht. In der Arkonasee reichen die Schwankungen bis $20 \mathrm{~m}$ hinunter. Überall stoßen die Maxima des Bodens und der Oberfläche aneinander, ohne eine Lücke freizulassen. Nur die zweiten Bodenmaxima gehen auf DO 1, DO 3 und DO 5 lediglich 5-10 $\mathrm{m}$ hoch. Ein Zwischenraum stellt sich in Samsöbelt mit Ausnahme von Da 22 und im südlichen Kattegat ein, er ist 10-15 m mächtig. Die Minima des Bodens gehen fast genau so hoch wie die Maxima. Nur im Fehmarnbelt und Alsenbelt sind sie bis $30 \mathrm{~m}$ Ticfe beobachtet, dafür bis $10 \mathrm{~m}$ nördlich der Kieler Förde. Die Minima der Oberfläche gehen hingegen nicht tief, der größte Teil 5-10 m, einige machen sich nur an der Oberfläche bemerkbar, hingegen verlaufen sie in der Arkonasee bis $30 \mathrm{~m}$. Sie lassen eine deutliche Lücke gegenüber dem Minimum des Bodens. 
Dinnit ist keineswegs festgestellt, daß hier eine Zwischenschicht existiert, da die Maxima sich an der Oberfläche und an Boden in der Regel nicht einander entsprechen. Nur von Sprogï an bis gegen das Südendo des Großen Beltes existiert eine gewisse GesetzmäBigkeit, indem, wis wir schon bemerkten und die beigegebene Tabelle zeigt, einem winterlichen Oberflächenmaximum ein Bodenminimum parallel geht, einem sommerlichen Oherflächenminimum ein Borlenmaximum. Bei dem Zusammentreffen der beiden letzten ereignet es sich dann, daß eine Zwischenschicht von reichlich $10 \mathrm{~m}$ Mächtigkeit eingeschaltet ist, die weder den Veränderungen am Boden noch denen an der Oberfläche ummittelbar folgt. Hier verschieben sich die Extreme auf andere Jahreszeiten, doch ist in den Beobachtungen der vier Stationen keine RegelmäBigkeit zu erkenner.

\section{Tabelle 7. Salzgehaltsmittel von November 1902 bis August 1906 für die internationalen Terminstationen.}

\section{GotIandsee.}

Salzgehalt in $\%$.

DO 10. $54^{0} 35^{\prime} 15^{0} 30^{\prime}$

\begin{tabular}{|c|c|c|c|c|c|}
\hline $\begin{array}{l}\text { Jahreszeit .. } \\
\text { Datum ... }\end{array}$ & $\begin{array}{l}\text { Winter } \\
\text { 4. II.-19. II. }\end{array}$ & $\begin{array}{c}\text { Frühjahr } \\
\text { 3. V.-12. V. }\end{array}$ & $\begin{array}{c}\text { Sommer } \\
\text { 4. VIII.-16. VIII. }\end{array}$ & $\begin{array}{c}\text { Herbst } \\
-\end{array}$ & Jahresmittel \\
\hline $\begin{array}{r}0 \mathrm{~m} \\
5 \% " \\
10 " \\
15 " \\
20 " \\
30 " \\
40 " \\
50 " \\
60 "\end{array}$ & $\begin{array}{r}7,52 \\
7,53 \\
7,53 \\
7,57 \\
7,41 \\
7,53 \\
7,54 \\
8,14 \\
13,33\end{array}$ & $\begin{array}{r}7,44 \\
7,40 \\
7,43 \\
7,42 \\
7,44 \\
7,47 \\
7,54 \\
8,42 \\
12,71\end{array}$ & $\begin{array}{r}7,31 \\
7.30 \\
7,31 \\
7,32 \\
7,34 \\
7,36 \\
7,45 \\
8.91 \\
12,79\end{array}$ & $\begin{array}{l}- \\
- \\
- \\
- \\
- \\
-\end{array}$ & $\begin{array}{l}- \\
- \\
- \\
- \\
-\end{array}$ \\
\hline
\end{tabular}

\section{Arkonasee.}

Salzgehalt in $\%$.

DO Q. $54^{\circ} 44^{\prime} 13^{\circ} 17^{\prime}$

\begin{tabular}{|c|c|c|c|c|c|}
\hline $\begin{array}{l}\text { Jahreszeit . } \\
\text { Datum . . }\end{array}$ & $\begin{array}{l}\text { IVinter } \\
\text { 1. II.-18. II. }\end{array}$ & $\begin{array}{l}\text { Frühji hi } \\
\text { 3. V.-11.V. }\end{array}$ & $\begin{array}{l}\text { Sommer } \\
\text { 3. VIII. - 15. VIII. }\end{array}$ & $\begin{array}{c}\text { Herbst } \\
\text { 31.X.-17.XI. }\end{array}$ & Jahresmittel \\
\hline $\begin{array}{r}\text { Tiefen: } \\
5 \\
10 " \\
15 " \\
20 " \\
30 " \\
34 "\end{array}$ & $\begin{array}{r}8,50 \\
8,53 \\
9,74 \\
9.86 \\
10,88 \\
12,20 \\
12,28\end{array}$ & $\begin{array}{r}7,73 \\
7,75 \\
8,14 \\
8,31 \\
8.78 \\
9,70 \\
10,36\end{array}$ & $\begin{array}{r}7,81 \\
7.81 \\
8,15 \\
8.60 \\
9,23 \\
12,65 \\
13,48\end{array}$ & $\begin{array}{r}7,76 \\
7,75 \\
7,79 \\
7,86 \\
8,31 \\
9, \tilde{5} 8 \\
10,65\end{array}$ & $\begin{array}{r}7,95 \\
7,96 \\
8,46 \\
8,66 \\
9,30 \\
11,03 \\
11,69\end{array}$ \\
\hline
\end{tabular}


00 8. $5 t^{0} 5 t^{\prime} 13^{\circ} 12^{\prime}$

\begin{tabular}{r|c|c|c|c|c}
\hline Jahreszeit . & $\begin{array}{c}\text { Winter } \\
\text { Datum . . . }\end{array}$ & $\begin{array}{c}\text { Frühjihr } \\
\text { 1. II.-18. II. }\end{array}$ & $\begin{array}{c}\text { Sommer } \\
\text { 3. V.-11. V. }\end{array}$ & $\begin{array}{c}\text { Herbst } \\
\text { 3. VIII.-15. VIII. 31.X.—17. XI. }\end{array}$ & Jahresmittel \\
\hline Tiefen: 0 m & 8,22 & 7,60 & 7,68 & 7,99 & 7,87 \\
$5 \%$ & 8.15 & 7.62 & 7,66 & 8,00 & 7,86 \\
$10 "$ & 8,19 & 7,64 & 7,66 & 8,00 & 7,87 \\
$15 "$ & 8,41 & 7,64 & 7,70 & 7,99 & 7,94 \\
$20 "$ & 8,45 & 7,76 & 7,81 & 8,00 & 8,01 \\
$30 "$ & 10,11 & 9,20 & 10,28 & 8,42 & 9,50 \\
$40 "$ & 14.11 & 12.28 & 14,19 & 9,48 & 12,52 \\
$44 "$ & 16,84 & 15,40 & 15,70 & 12,46 & 15,10
\end{tabular}

D० 7. $55^{0} 06^{\prime} 13^{0} 06^{\prime}$

Datum . . . .| 2. II.-18. II. 3. V.-11. V. 3. VIII.-15. VIII 31.X.-16.XI.

\begin{tabular}{rr|r|r|r|r}
\hline \hline Tiefen: $0 \mathrm{~m}$ & 8,39 & 7,76 & 7,61 & 7,74 & \multicolumn{1}{c}{7,88} \\
$5 \%$ & 8,37 & 7.74 & 7,63 & 7,75 & 7,87 \\
$10 "$ & 8.37 & 7,76 & 7,65 & 7,77 & 7,89 \\
$15 "$ & 8,49 & 7,76 & 7,63 & 7,78 & 7,90 \\
$20 "$ & 8,70 & 7,81 & 7,77 & 7,90 & 8,05 \\
$30 "$ & 11,12 & 8,37 & 10,17 & 9,01 & 9.67 \\
$37 "$ & 14,89 & 11,62 & 14,28 & 13,15 & 13,49
\end{tabular}

DO 6. $55^{0} 16^{\circ} 513^{\circ} 01^{\prime}$

Datum . . . || 2. II.-17. II. 3. V.-11. V. 3. VIII - 15. VIII. 31.X.-16 XI.

\begin{tabular}{r|r|r|r|r|r}
\hline \hline Tiefen: $0 \mathrm{~m}$ & 8,77 & 7,61 & 7,91 & 7,65 & 7,99 \\
5 & 8,80 & 7,58 & 7,90 & 7,64 & 7,98 \\
$10 "$ & 8,90 & 7,59 & 7.91 & 7,63 & 8,01 \\
$15 "$ & 9,14 & 7.60 & 8,04 & 7,64 & 8,11 \\
$20 "$ & 9,49 & 7.61 & 7,92 & 7,65 & 8,17 \\
$29 "$ & 10,52 & 7,63 & 8,51 & 7,72 & 8,60
\end{tabular}

Stammbelt.

Salzgehalt in $\%$.

DO 5. $54^{0} 28^{\prime} 12^{\circ} 15^{\prime}$

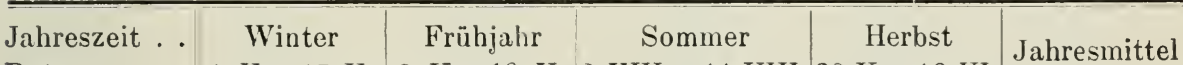

Datum .... 1. II.-17.II. 2. V.-10. V. 2. VIII.-14. VIII.30.X.-16. XI.

\begin{tabular}{rr|r|r|r|r|r}
\hline Tiefen : $0 \mathrm{~m}$ & 12,60 & 8,53 & 8,47 & 10,23 & 9,46 \\
5 & 12,66 & 8,54 & 9,25 & 10,15 & 10,15 \\
$10 "$ & 13,35 & 9,73 & 11,95 & 10,59 & 11,16 \\
15 & 15, & 15,61 & 15,04 & 14,93 & 11,51 & 14,27 \\
$20 "$ & 16,74 & 16,33 & 16,87 & 12,35 & 15,57 \\
$26 "$ & 17,98 & 16,99 & 18,82 & 13,65 & 16,86
\end{tabular}

Da 32. $54^{0} 35^{\prime} 511^{\circ} 03^{\prime} 5$

\begin{tabular}{|l|l|l|} 
Datum .... & 1. V.-3. V. & 1. VIII.-3. VIII. $\mid$ 2. XI.-3.XI.
\end{tabular}

\begin{tabular}{r|r|r|r|r|r}
\hline \hline Tiefen : $0 \mathrm{~m}$ & - & 12,34 & 12,70 & 13,41 & - \\
5 & - & 12,63 & 13,90 & 13,64 & - \\
$10 "$ & - & 15,15 & 13,17 & 14,58 & - \\
$15 "$ & - & 17,20 & 16,99 & 15,80 & - \\
$20 "$ & - & 18,95 & 19,30 & 17,67 & - \\
$30 "$ & - & 20,54 & 25,48 & 20,26 & -
\end{tabular}


$003.54^{\circ}: 3\left(3^{\prime} 11^{\circ} 02^{\circ}\right.$

\begin{tabular}{|c|c|c|c|c|c|}
\hline $\begin{array}{l}\text { Jahreszeit . . } \\
\text { Datum ... }\end{array}$ & $\begin{array}{l}\text { Wintor } \\
\text { 1. 11. }-17.11 .\end{array}$ & $\begin{array}{l}\text { Frühịiahr } \\
\text { 2. V. S. V. }\end{array}$ & $\begin{array}{c}\text { Sommer } \\
\text { 2. V111.-11. V111. }\end{array}$ & $\begin{array}{c}\text { Herlst } \\
\text { 3().X-15 XI. }\end{array}$ & Jihresmittel \\
\hline $\begin{array}{r}\text { Tiefen: } 0 \text { m } \\
5 \% " \\
10 " \\
15, " \\
20 " \\
30 " \\
30 "\end{array}$ & $\begin{array}{l}17.07 \\
17.07 \\
18.30 \\
21.95 \\
20.4 ! \\
21.8 .5 \\
2.219\end{array}$ & $\begin{array}{l}13,26 \\
13,77 \\
14,98 \\
16,1 \% \\
18,23 \\
20,1: 3 \\
2(1,29)\end{array}$ & $\begin{array}{l}13.02 \\
13.38 \\
15.52 \\
16,81 \\
20,54 \\
27,06 \\
27,49\end{array}$ & $\begin{array}{l}12 .(i 2 \\
12,(6 i 3 \\
13,28 \\
15,31 \\
17,12 \\
20,5 \% 3 \\
20,42\end{array}$ & $\begin{array}{l}1399 \\
14,21 \\
15.52 \\
17,56 \\
19,10 \\
2.2,39 \\
22,60\end{array}$ \\
\hline
\end{tabular}

\section{Iiibecker Bucht.}

Salzgehalt in $\%$.

DO 4. $54^{\circ} 10^{\prime} 11^{\circ} 16^{\prime}$

\begin{tabular}{|c|c|c|c|c|c|}
\hline $\begin{array}{l}\text { Jalıreszeit . . } \\
\text { I)atum . . . }\end{array}$ & $\begin{array}{l}\text { IVinter } \\
\text { 1. II. }-17 . \text { II. }\end{array}$ & $\begin{array}{l}\text { Frülijahr } \\
\text { 2. V.-10. V. }\end{array}$ & $\begin{array}{l}\text { Sommer } \\
\text { 2. VIII.-14.VIII. }\end{array}$ & $\begin{array}{c}\text { Herbst } \\
30 . \mathrm{X}-16 \mathrm{XI} .\end{array}$ & Jahresmittel \\
\hline $\begin{array}{r}0 \mathrm{~m} \\
5 \\
10 " \\
15 " \\
20 " \\
2.2 "\end{array}$ & $\begin{array}{l}15,50 \\
15.52 \\
16.06 \\
16.23 \\
17,30 \\
17,79\end{array}$ & $\begin{array}{l}11,90 \\
12,39 \\
14,41 \\
16,09 \\
17,56 \\
18,19\end{array}$ & $\begin{array}{l}10.91 \\
10.92 \\
12,20 \\
14,95 \\
20,84 \\
21,80\end{array}$ & $\begin{array}{l}12,65 \\
12,70 \\
13,32 \\
15,41 \\
17,23 \\
17,86\end{array}$ & $\begin{array}{l}12,74 \\
1:, 88 \\
14,00 \\
15,67 \\
18,23 \\
18,91\end{array}$ \\
\hline
\end{tabular}

Kieler Bucht.

Salzgehalt in ${ }^{0} / 00$.

DO 1. $54^{\circ} 29^{\prime} 510^{\circ} 21^{\prime}-22^{\prime}$

\begin{tabular}{|c|c|c|c|c|c|}
\hline $\begin{array}{l}\text { Jahreszeit . } \\
\text { Datım . . . }\end{array}$ & $\begin{array}{l}\text { Winter } \\
\text { 30. I.-16. II }\end{array}$ & $\begin{array}{l}\text { Frūhijahr } \\
\text { 1. V.-9. V. }\end{array}$ & $\begin{array}{l}\text { Sommer } \\
\text { 1. VIII.-13. VIII. }\end{array}$ & $\begin{array}{c}\text { Herbst } \\
\text { 29.X.-14.XI. }\end{array}$ & Jahresmi \\
\hline $\begin{array}{r}\text { Tiefen: } 0 \mathrm{~m} \\
5 \% " \\
10 " \\
15 " \\
19 "\end{array}$ & $\begin{array}{l}17,85 \\
17,82 \\
18,57 \\
19,62 \\
20,05\end{array}$ & $\begin{array}{l}15,18 \\
15,16 \\
16,40 \\
18,50 \\
19,39\end{array}$ & $\begin{array}{l}13.56 \\
13.61 \\
15.59 \\
19,26 \\
20,34\end{array}$ & $\begin{array}{l}15,27 \\
15,25 \\
15,37 \\
16,27 \\
16,65\end{array}$ & $\begin{array}{l}15,47 \\
15,46 \\
16,48 \\
18,41 \\
19,11\end{array}$ \\
\hline
\end{tabular}

Kleiner Belt.

Salzgehalt in $\%$.

DO 2. $5 t^{0} 56^{\prime} 10^{0} 1 ?^{\prime}$

\begin{tabular}{|c|c|c|c|c|c|}
\hline $\begin{array}{l}\text { Jahreszeit . . } \\
\text { Datum ... }\end{array}$ & $\begin{array}{l}\text { Winter } \\
\text { 31.I.-16. II. }\end{array}$ & $\begin{array}{c}\text { Frühįahr } \\
\text { 2. V.-10. V. }\end{array}$ & $\begin{array}{c}\text { Sommer } \\
\text { 2.VIII-13.VIII. }\end{array}$ & $\begin{array}{c}\text { Herbst } \\
29 \mathrm{X} .-15 . \mathrm{XI} .\end{array}$ & Jahresmittel \\
\hline $\begin{array}{r}\text { Tiefen: } 0 \mathrm{~m} \\
5 \\
10 " \\
15 " \\
20 " \\
30 " \\
33 "\end{array}$ & $\begin{array}{l}19,21 \\
19,16 \\
19,37 \\
19,57 \\
19,70 \\
20,74 \\
21,88\end{array}$ & $\begin{array}{l}15,50 \\
15,47 \\
16,23 \\
18,23 \\
19,33 \\
21,15 \\
22,40\end{array}$ & $\begin{array}{l}14.64 \\
14,72 \\
16,80 \\
19.04 \\
20,89 \\
24,82 \\
25,12\end{array}$ & $\begin{array}{l}15,96 \\
15,94 \\
16,31 \\
17,10 \\
17,73 \\
21,35 \\
22,29\end{array}$ & $\begin{array}{l}16,33 \\
16,32 \\
17,18 \\
18,49 \\
19,91 \\
22,02 \\
22,92\end{array}$ \\
\hline
\end{tabular}




\section{Großer BeIt.}

Salzgehalt in $\%$.

Dа 31. $54^{0} 43^{\prime} 910^{\circ} 46^{\prime} \ddagger$

\begin{tabular}{|c|c|c|c|c|c|}
\hline $\begin{array}{l}\text { Jahreszeit . } \\
\text { Datum ... }\end{array}$ & $\begin{array}{l}\text { Winter } \\
\text { 2. II.-3. II. }\end{array}$ & $\begin{array}{l}\text { Frühiahr } \\
1 . \mathrm{V}-2 . \mathrm{V} .\end{array}$ & $\begin{array}{l}\text { Sommer } \\
\text { 1. VIII-2. VIII. }\end{array}$ & $\begin{array}{c}\text { Herbst } \\
\text { 2.XI-3. XI. }\end{array}$ & Jahresmittel \\
\hline $\begin{array}{r}\text { Tiefen: } 0 \text { m } \\
5 " n \\
10 " \\
15 " \\
20 " \\
30 "\end{array}$ & $\begin{array}{l}19,70 \\
19,76 \\
19,80 \\
19,84 \\
19,85 \\
21,29\end{array}$ & $\begin{array}{l}11,83 \\
13,17 \\
13,94 \\
16,80 \\
20,83 \\
24.57\end{array}$ & $\begin{array}{l}12,12 \\
15,06 \\
13.37 \\
15,68 \\
23,48 \\
28,83\end{array}$ & $\begin{array}{l}14,40 \\
14,49 \\
15.00 \\
16,16 \\
20,03 \\
22,04\end{array}$ & $\begin{array}{l}14,51 \\
15.62 \\
15.53 \\
17,12 \\
21,05 \\
24,18\end{array}$ \\
\hline
\end{tabular}

D а 3०. $54^{0} 52 ‘ 510^{0} 50^{\prime} \check{0}$

Datum ....|| 2. II.--3. II. 1. V.-2. V. 1. VIII - 2. VIII. 2.XI-3.XI.

\begin{tabular}{r|r|r|r|r|r}
\hline \hline Tiefen: $0 \mathrm{~m}$ & 20,07 & 12,29 & 12,31 & 14,45 & 14,79 \\
5 & 20,17 & 13.12 & 13,07 & 14,66 & 15,26 \\
$10 "$ & 20,18 & 14.87 & 15,56 & 15,58 & 16.55 \\
$15 "$ & 20,19 & 15,44 & 18,29 & 19,14 & 18,27 \\
$20 "$ & 20,22 & 20,32 & 27,04 & 20,33 & 21,98 \\
$30 "$ & 20,26 & 24,31 & 28,46 & 23,36 & 24,10 \\
$40 "$ & 21.27 & 24,91 & 28,69 & 25,00 & 24,97 \\
$50 "$ & 21,29 & 25,13 & 28,94 & 25,14 & 25,13
\end{tabular}

Da 29. $55^{\circ} 01^{\prime} 511^{0} 02^{\prime} 2$

Datum ... . || 2. II.-3. II. 1. V.-2. V. 1. VIII.-2. VIII. 1.XI.-3.XI.

\begin{tabular}{rr||l|l|l|l}
\hline \hline Tiefen: $0 \mathrm{~m}$ & 19,76 & 12,91 & 12,28 & 14,81 & 14.94 \\
5 & 19,80 & 13,36 & 12,71 & 14,98 & 15,21 \\
$10 "$ & 20,24 & 14,02 & 13,69 & 15,34 & 15,82 \\
$15 "$ & 20,30 & 15,91 & 16,64 & 19,85 & 18,18 \\
$20 "$ & 20,80 & 22.38 & 26,77 & 22,39 & 23,09 \\
$: 0 "$ & 23,93 & 26,27 & $30,2 \pm$ & 27,03 & 26,87
\end{tabular}

Da 28. $55^{0} 11^{\prime} 911^{0} 05^{\prime} 3$

Datum .... 2. II. -3. II. 1. V.-2. V. 1.VIII-2.VIII. 1.XI.-2. XI.

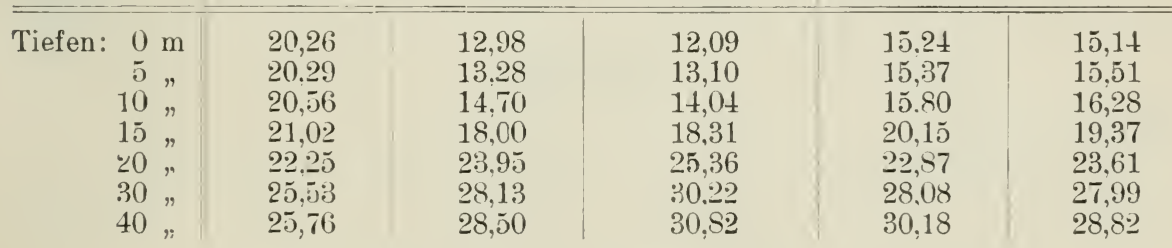

Da 27. $55^{\circ} 19^{\prime} 210^{0} 53^{\prime} 4$

Datum.... 1. II. 1. V.-2. V. 1. VIII.-2. VIII. 1. XI.

\begin{tabular}{r|r|r|r|r|r|}
\hline Tiefen: $0 \mathrm{~m}$ & 20,97 & 14,78 & 13.23 & 15,66 & 16,16 \\
5 & 21.17 & 15.14 & 14,51 & 15,98 & 16,70 \\
$10 "$ & 21,76 & 16,50 & 16,80 & 18,99 & 18,51 \\
$15 "$ & 22,60 & 21,85 & 22,24 & 23.42 & 22.53 \\
$20 "$ & 23,96 & 26,25 & 27.98 & 26.73 & 26,23 \\
$25 "$ & 25,04 & 28,03 & 30,95 & 29.86 & 28,47
\end{tabular}


Da 26.5$\left.)^{0} \div 0^{\prime}+11^{\prime \prime}(1)^{\prime}\right)^{\prime} 3$

\begin{tabular}{|c|c|c|c|c|c|c|}
\hline $\begin{array}{l}\text { Jahresze } \\
\text { Datum }\end{array}$ & eiten & $\begin{array}{l}\text { Winter } \\
\text { 1. II.-2. II. }\end{array}$ & $\begin{array}{l}\text { Frühjahr } \\
\text { I. V.-?. V. }\end{array}$ & $\begin{array}{l}\text { Sommer } \\
\text { 1. VIII.-2. VIII. }\end{array}$ & $\begin{array}{c}\text { Herbst } \\
\text { 1. XI. - 2. XI. }\end{array}$ & Jahresmittel \\
\hline Tiefen: & $\begin{array}{ll}0 & \mathrm{~m} \\
5 & \\
10 & " \\
15 & " \\
25 & " \\
30 & " \\
40 & " \\
50 & " \\
55 & "\end{array}$ & $\begin{array}{l}19,31 \\
20.14 \\
20,68 \\
21,23 \\
24,52 \\
25,67 \\
26,36 \\
26,75 \\
27,80\end{array}$ & $\begin{array}{l}13,91 \\
14,69 \\
15,53 \\
19,89 \\
25,51 \\
28.07 \\
24,15 \\
28,46 \\
28,64\end{array}$ & $\begin{array}{l}12,64 \\
12,54 \\
14,01 \\
25,68 \\
30,02 \\
30,87 \\
30,98 \\
31,00 \\
30,97\end{array}$ & $\begin{array}{l}15,11 \\
15,50 \\
16,08 \\
19,94 \\
25,32 \\
28,22 \\
29,13 \\
29,70 \\
29,87\end{array}$ & $\begin{array}{l}15,25 \\
15,79 \\
16,58 \\
21,69 \\
26,34 \\
28,21 \\
28,66 \\
28,98 \\
29,32\end{array}$ \\
\hline
\end{tabular}

Da 25. $55^{\circ} 29^{\prime} 8 \quad 10^{\circ} 52^{\prime} 6$

\begin{tabular}{l|l|l|l|} 
Datum .... 1. II. & 1. V.-2.V. & 1.VIII.-2.VIII. & 1. XI.
\end{tabular}

\begin{tabular}{r|r|r|r|r|r}
\hline \hline Tiefen: $0 \mathrm{~m}$ & 21,72 & 14,61 & 13,87 & 16,08 & 16,57 \\
5 & 21,83 & 15,24 & 14.82 & 16,40 & 17,07 \\
$10 \%$ & 22,18 & 16,84 & 15,87 & 17,99 & 18,22 \\
15 & 22,94 & 21,84 & 20,77 & 21,73 & 21,82 \\
$20 "$ & 25,55 & 27,06 & 28,79 & 28,14 & 27,38 \\
$30 "$ & 26,92 & 30,60 & 31,02 & 31,14 & 29,92
\end{tabular}

Da 24. $55^{\circ} 36^{\prime} 610^{\circ} 48^{\prime} 8$

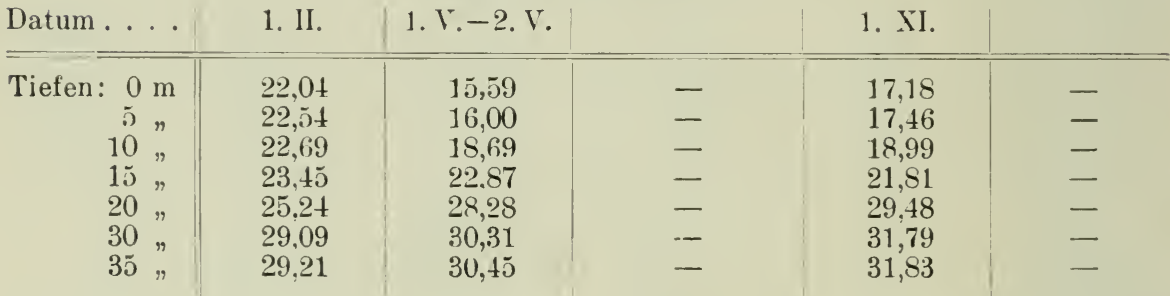

Samsöbelt.

Salzgehalt $\%$

Da 23. $55^{\circ} 46^{\prime} 710^{\circ}+8^{\prime} 8$

\begin{tabular}{|c|c|c|c|c|c|}
\hline $\begin{array}{l}\text { Jahreszeiten } \\
\text { Datum . . . }\end{array}$ & $\begin{array}{l}\text { Winter } \\
\text { 1. II. }-4 . \text { II. }\end{array}$ & $\begin{array}{l}\text { Frūhijahr } \\
\text { 1. V.-2. Ir. }\end{array}$ & $\begin{array}{l}\text { Sommer } \\
\text { 1. VIII-3. VIII. }\end{array}$ & $\begin{array}{c}\text { IIerbst } \\
\text { 1. XI.-3. XI. }\end{array}$ & Jahresmittel \\
\hline $\begin{array}{r}\text { Tiefen: } 0 \mathrm{~m} \\
5 \\
10 " \\
15 " \\
20 " \\
30 " \\
40 " \\
50 "\end{array}$ & $\begin{array}{l}21,00 \\
21,69 \\
22,49 \\
23,75 \\
25,89 \\
28,66 \\
29,17 \\
29,63\end{array}$ & $\begin{array}{l}15.49 \\
15.83 \\
16,25 \\
23.23 \\
28,80 \\
30.71 \\
30.61 \\
30,68\end{array}$ & $\begin{array}{l}13,66 \\
13,93 \\
18,10 \\
26,11 \\
30,09 \\
30,91 \\
31,17 \\
31,34\end{array}$ & $\begin{array}{l}17,05 \\
17,09 \\
19,01 \\
23,45 \\
29,7 \cdot 2 \\
31,06 \\
31,61 \\
32,06\end{array}$ & $\begin{array}{l}16,80 \\
17,14 \\
18.96 \\
24,14 \\
28,63 \\
30,34 \\
30,64 \\
20,93\end{array}$ \\
\hline
\end{tabular}


Da 22, $55^{0} 56^{\prime} 911^{\circ} 00^{\prime} 2$

\begin{tabular}{c|c|c|c|c|c}
\hline $\begin{array}{c}\text { Jahreszeiten } \\
\text { Datum. . . }\end{array}$ & $\begin{array}{c}\text { Winter } \\
\text { 1. II.4. II. }\end{array}$ & $\begin{array}{c}\text { Frühjahr } \\
\text { 2. V. }\end{array}$ & $\begin{array}{c}\text { Sommer } \\
\text { 1. VIII.-2. VIII. }\end{array}$ & $\begin{array}{c}\text { Herbst } \\
\text { 2. XI.-3. XI. }\end{array}$ & Jahresmittel \\
\hline \hline Tiefen: 0 m & 21,70 & 16,11 & 14,83 & 17,66 & 17,58 \\
$5 "$ & 21,78 & 17,06 & 15,00 & 17,76 & 17,90 \\
$10 "$ & 23,14 & 19,06 & 17,46 & 21,62 & 20,32 \\
$15 "$ & 26,16 & 28,60 & 27,52 & 29,07 & 27,84 \\
$20 "$ & 29,52 & 30,59 & 29,95 & 31,64 & 30,43 \\
$30 "$ & 30,07 & 31,41 & 30,70 & 32,51 & 31,17 \\
$40 "$ & 30,87 & 31,50 & 31,18 & 32,76 & 31,58 \\
$50 "$ & 31,19 & 31,55 & 31,42 & 32,93 & 31,77
\end{tabular}

Da 21. $56^{0} 07^{\prime} 911^{0} 11^{\prime} 1$

Datum....|| 1. II.-4. II. $\quad$ 2. V. | 1. VIII.-2. VIII. 2. XI.-3. XI.

\begin{tabular}{c||c|c|c|c|c}
\hline \hline Tiefen: $0 \mathrm{~m}$ & 22,52 & 17,51 & 15,27 & 18,45 & 18,44 \\
$5 \%$ & 22,68 & 18.41 & 16,35 & 19,01 & 19,12 \\
$10 "$ & 23,47 & 19,39 & 17,26 & 19,68 & 19,95 \\
$15 "$ & 26,33 & 25,82 & 26.07 & 27,26 & 26,37 \\
$20 "$ & 30,83 & 30,50 & 30,69 & 32,44 & 31,13 \\
$30 "$ & $31, \cdots 5$ & 32,91 & 31,68 & 33,36 & 32,30 \\
36, & 31,37 & 32,99 & 31,72 & 33,45 & 32,38
\end{tabular}

\section{Kattegat.}

Salzgehalt $\%$.

Da 20. $56^{\circ} 22^{\prime} 311^{\circ} 48^{\prime} 6$

\begin{tabular}{l||c|c|c|c|c}
\hline \hline Jahreszeiten & Winter & Frühjuhr & Sommer & Herbst & Jahresmittel \\
Datum . . . . & 1. II.-4. II. & 1. V.-2. V. & 1. VIII.-3. VIII. & 1. XI.-3. XI. &
\end{tabular}

\begin{tabular}{r||r}
\hline \hline Tiefen: $0 \mathrm{~m}$ & 22,97 \\
$5 "$ & 23,43 \\
$10 "$ & 24,31 \\
$15 "$ & 25,44 \\
$20 "$ & 28,09 \\
$27 "$ & 31,73
\end{tabular}

\begin{tabular}{l|l}
17,66 & 16,17 \\
19,26 & 19,48 \\
20,54 & 19,92 \\
27,21 & 22,25 \\
32,84 & 27,36 \\
33,27 & 32,04
\end{tabular}

19,38
19,44
20,36
22,14
30,75
33,84

19,05

20,15

21,28

24.26

29.76

32,72

Da 19. $56^{0} 35^{\prime}+11^{\circ} 51^{\prime} 3$

\begin{tabular}{l|l|l|} 
Datum .... & 1.V.-2. V. $\mid$ X. XI.-3.XI.
\end{tabular}

\begin{tabular}{r||l|l|l|l|l}
\hline Tiefen: $0 \mathrm{~m}$ & - & 17,90 & - & 19,13 & - \\
5, & - & 18,35 & - & 19,39 & - \\
$10 "$ & - & 20,61 & - & 20,29 & - \\
$15 "$ & - & 27,01 & - & 21,59 & - \\
$20 "$ & - & 29,87 & - & 30,24 & - \\
$30 "$ & - & 33,46 & - & 33,89 & -
\end{tabular}

Da 18. $56^{\circ} 47^{\prime} 311^{\circ} 47^{\prime} 0$
Datum .
I. $\mathrm{V}$.
1. VIII.
1. XI.-3.XI.

\begin{tabular}{r||l|l|l|l|l}
\hline \hline Tiefen : 0 m & 24,72 & 20,28 & 19,13 & 20,28 & 21,10 \\
$5 "$ & 24,78 & 20,76 & 20,28 & 20,40 & 21,56 \\
$10 "$ & 25,46 & 21,69 & 21,27 & 22,16 & 22,65 \\
$15 "$ & 28,73 & 29,69 & 27,05 & 27,10 & 28,14 \\
$20 "$ & 31,12 & 32,84 & 30,04 & 31,37 & 31.34 \\
$30 "$ & 32,73 & 33,65 & 31,76 & 33,83 & 32,99 \\
$40 "$ & 33,31 & 33,97 & 33,05 & 33,81 & 33,54 \\
$45 "$ & 33,45 & 34,08 & 33,09 & 33,91 & 33,63 \\
\end{tabular}




\section{Synoptisches Bild der Salzgehaltsverteilung.}

Dio Arkonaseo S. 92. - Die Funlitionen der Kactetrinne und des Sundes S. 95. Der Stammbelt S. :6. - Die Kieler Bucht S.9S. - Der Große Belt und der Samsö Belt S. 99. - Ias südliche Kintegat S. 160, - Tabellen S. 102.

Einige Detailzüge in den behandelten Wasserflächen zu gewinnen, gestatten die Beobachtungen, die seit 1907 in schnellerer Reihenfolge und mit Einschub neuer Stationen erfolgen. Ihre Positionen sind in die Skizze S. 72 eingetragen. Wir können Schnitte auf Grund von Serien konstruieren, die, was für unser Gebiet sehr wichtig und unbedingt notwendig ist, alle an einem einzigen Tage genommen sind. Am günstigsten stellt sich die Interpretation für die Arkonasee, wo sich die regelmäßigen Terminfahrten mit solchen außer der Reihe auf das glücklichste verbinden.

Für die Speisung der Gotlandsee mit ozeanischem Wasser ist der Schnitt Schonen-Rügen von größter Wichtigkeit. Er wird von Süden nach Norden durch die Stationen DO 9, DO 8, DO 7 und DO 6 verkörpert. Er ist schon oft behandelt worden, allerdings auf Grund eines weniger ausgiebigen Nateriales, und hervorragende Ozeanographen wie Krümmel haben sich spezieller mit ihm befaBt. Und doch ist auf allen Darstellungen ein meines Erachtens wichtiger morphologischer Zug fortgelassen, die Möenbank, die in seiner nördlichen Hälfte gelegen ist und den Ausgang des Arkonabeckens in zwei Arme teilt, wie ihn die Querschnitte auf Seite 93 wiedergeben.

Betrachten wir sie, so nehmen wir auf allen nördlich der Möenbank einen auffallend geringen Salzgehalt wahr. Ich habe mich nicht für berechtigt gehalten, Isohalinen, die auf der immerhin $29 \mathrm{~m}$ tiefen Station DO 6 nicht angetroffen waren, wohl aber bei DO 7, nördlich über die Möenbank hinauszuführen. Denn was könnte es für eine natürlichere Grenzfläche zwischen zwei so stark voneinander abweichenden und doch so nahen Stationen geben als eine Scheidewand, die sich auf über $20 \mathrm{~m}$ erhebt? Vergleicht man die Schnitte mit ihrer Lage in der Arkonasee, so ergibt sich sofort ungezwungen der Schluß, daß der Einlauf sich durch den Stammbelt südlich der Möenbank, der Auslauf nördlich von ihr bewegt. Studieren wir die Einzelheiten, so erkennen wir am Südrand ein Aufwärtsdrängen der Isohalinen, ein deutliches Merkmal, daß das einströmende Wasser sich besonders nahe der deutschen Küste bewegt. Daß diese Regel auch noch etwas weiter östlich gilt, zeigen einige Schnitte, die von schwedischer Seite auf den Stationen Ekman A 50-54 zwischen Arkona und Schonen genommen wurden. 
Namentlich am 15. Mai 1910 drängte sich das zahlreiche Wasser scharf an unsere Küste heran.

Nur im Winter folgt das Bodenwasser nicht dieser allgemeinen Erscheinung, wie uns verständlich wird, wenn wir erwägen, daß in dieser Jahreszeit auch durch den Sund ein Einlauf in stärkerem Maße erfolgt. Schon im Herbstschnitt ist dieser Bau angedentet. Im nördlichen Teilbecken finden wir in der Regel nur einen Salzgehalt zwischen 8 und $9 \%$; lediglich im Winter, wenn der Auslauf am reichsten und der Salzgehalt am höchsten ist, begegnen wir auch der 10\% Isohaline.

Ober die Verteilung in der Arkonasee können wir auf Grund des Schnittes Ekman A 50-54, der seit 1907 mehrfach wiederholt wurde und der etwas östlicher liegt als der deutsche Schnitt, noch ein genaucres Bild entworfen. 1907 zeigte sich sowohl Mitte Juli wie in den ersten Augusttagen ein gleicher Grundzug. Der hohe Salzgehalt lagert am Boden des Arkonabeckens. In Ekman A 50 unmittelbar nördlich Arkona war er nicht mehr zu bemerken, bis $35-39 \mathrm{~m}$ Tiefo wurde als Maximum $8,87^{\circ} /$ on gefunden; in gleicher Tiefe war auf dem nördlicher gelegenen Ekman A 52 auch noch kein größerer Salzgehalt zu konstatieren, sondern erst unter $40 \mathrm{~m}$ stellte er sich ein und bedeckte als dünne Lage von etwa $5 \mathrm{~m}$ Mächtigkeit den Boden. Auf dem schon höher gelegenen Ekman A 53 finden wir nur bis $9 \%$ am Boden, und an der Küste Schonens nur 8,13\% am Boden, also das süßere Auslaufwasser wieder nahe der schwedischen Küste. Bedeckt wird die ganze Südhälfte des Schnittes von homohalinem Wasser mit 7,52/00, das bei Ekman A $5131 \mathrm{~m}$ mächtig ist. Daß häufig an der Oberfläche nahe der Südseite das salzreiche Wasser angetroffen wird, zeigt auch ein Schnitt vom Dornbusch auf Hiddensö nach der Südostküste Möens vom 18. Juli 1907. In der Tiefe tritt dagegen dieser Zug nicht so klar hervor.

Im einzelnen kann dieses Bild mannigfach gestört werden. So treffen wir am 2. Februar 1905 auf DO 6 einen Salzgehalt an, der ron der Oberfläche bis zum Boden in $29 \mathrm{~m}$ Tiefe zwischen 10,81\% und $14,04^{\%}$ oo gelegen ist, eine Steigerung, wie sie nie wieder beobachtet wurde. In den letzten Tagen hatte ein einlaufender Strom bei Drogden hohe Salzgehaltswerte gebracht, am Morgen des 1. Februar sogar $20,0^{\circ}{ }_{00}$. Ein ähnlicher Fall für die gesamte Wassersäule liegt nicht mehr vor, wohl begegnet uns für den 17. Februar 1903 und 14. August 1904 ein starkes Anwachsen des Salzgehaltes am Boden, das sogar $20,55^{\circ}$ ion erreicht; beidemal hatte vorher Einlauf durch den Sund geherrscht, bei dem in dem einen Falle sogar Wasser von $27,1{ }^{\circ}$ on über die Drogdenschwelle gelangt war. 
Umgekehrt können wir bei DO 7 in der ganzen Wassersäule auf sehr niedrigen Salzgehalt treffen; am 3. November 1905 maß man am Boden nur $7,63^{\circ}$, ein Wert, der bei DO 8 auf $8,33^{\circ}, 00$ stieg und bei DO 9 erst $10,73^{\circ}{ }_{00}$ erreichte. Die Verteilung beweist, daß in diesem Becken für sich gleichfalls auf der einen Seite Einlauf, auf der anderen Auslauf erfolgen kann, mit Rechtsdrängung infolge der Erdrotation, wie wir noch vielfach wiedersehen werden. Auch das umgekehrte Bild vermag sich zu entwickeln, wie am 31. Oktober 1902, wo man am Boden von DO $98,28^{\circ}{ }_{00}$ maB, bei DO $813,86^{\circ} / 00$ und bei DO $713,25 \%$, nördlich der Möenbank dagegen nur $8,08 \%$, eine Verteilung, wie sie ja schon in der Mittelbildung zum Ausdruck kam. Auf allzu kleine Salzgehaltsunterschiede dürfen wir hierbei kein Gewicht legen, da die Beobachtungen nicht genau gleichzeitig erfolgen, sondern sich in der Regel über einen Tag erstrecken. Dagegen scheint vom Sommer bis Herbst sich gern nördlich von Rügen eine mächtige, bis zum Boden reichende Süßwasserschicht einzustellen, wie namentlich der Schnitt Ekman A $50-54$ vom 19. Juli sehr schön dartut.

$\mathrm{DaB}$ ein abnorm hoher Salzgehalt aber nicht nur seinen Weg durch den Sund genommen zu haben braucht, sondern auch durch die Kadetrinne gefunden haben kann, zeigt ein Blick auf die Salzgehaltswerte am Boden der Kadetrinne. Wir führten schon auf, daB hier fast $25 \%$ erreicht werden kann! Dieses salzreiche Wasser sinkt nach Passieren der Darsserschwelle natürlich sofort in die Tiefe des Arkonabeckens. Wir können nicht den jeweiligen Salzgehalt an beiden Orten miteinander vergleichen und, wenn wir am Boden der Arkonasee einen hohen Wert finden, nicht aber am Boden der Kadetrinne, den Schluß ziehen, das Bodenwasser der Arkonasee müsse den Sund passiert haben. Die Zufuhr durch die Kadetrinne kann schon wieder aufgehört haben, während das salzreiche Wasser noch am Boden der Arkonasee lagert. Der ausführlich besprochene rasche Wechsel des Salzgehaltes auf den Stationen der dänischen Feuerschiffe gibt uns hierfür einen wichtigen Anhalt.

Daß das Einlaufwasser sich am Boden längere Zeit fest halten kann, zeigt sein häufiges scharfes Absetzen gegen das Auslaufwasser. Schon Gehrke hat auf die große Steigerung aufmerksam gemacht, die sich in den letzten vier Metern vollziehen kann. Die häufig gefundene scharfe 'Trennung deutet auch darauf hin, daß die Durchmischung infolge von Stürmen in der Regel nicht bis zum Boden reicht. Als Beispiel mögen zwei Serien von DO 6 vom 12. und 14. August 1904 dienen, zwischen denen sich ein westlicher Sturm entwickelte, der au 
Gjeder Rer während drei Wachen, auf Drogden während zwei Warhen Sturmstärke 7 und mehr erreichte. I)ie Verhältnisse waren folgende:

\begin{tabular}{|c|c|c|c|c|c|c|}
\hline & $0 \mathrm{~m}$ & $5 \mathrm{~m}$ & $10 \mathrm{~m}$ & $15 \mathrm{~m}$ & $20 \mathrm{~m}$ & $29 \mathrm{~m}$ \\
\hline $\begin{array}{l}\text { 12. August } 1904 \\
\text { 14. August } 1904\end{array}$ & $\begin{array}{l}8,160^{0} 00 \\
\left.8,1,2^{0}\right)_{0}\end{array}$ & $\begin{array}{l}8, i \cup \% \\
8,10^{\prime \prime}\end{array}$ & $\begin{array}{l}8,12^{0}{ }_{00} \\
7,97^{\circ} 1_{00}\end{array}$ & $\begin{array}{l}8,24^{0 / 00} \\
8,12^{0 /} 00\end{array}$ & $\begin{array}{l}7.85 \% \% \\
5,33: 3 \% 00\end{array}$ & $\begin{array}{r}9,04^{\prime \prime} \% 00 \\
10,70^{\circ} \%\end{array}$ \\
\hline
\end{tabular}

Wir sehen die Einmischung des Sturines in Zufuhr salzreichen Wassers in der Tiefe.

Daß auch der Sund hieran beteiligt zu sein vermag, ohno daß es auf Feuerschiff Drogden beobachtet werden kann, zeigen einige wenige Serien aus dieser Wasserstraße, die unsere Ergebnisse für Drogden ergänzen. Am j. August 1907 wurden von der schwedischen Seite eine Serie in der Flintrinne und zwei dicht aufeinander folgende bei Helsingborg genommen. In der Flintrinne zeigte sich von $0 \mathrm{~m}$ bis $9,5 \mathrm{~m}$ Tiefe ein zwischen 8,50" $\ldots$ und 8,59\% ${ }^{\circ}$ schwankender Salzgehalt. Auf Drogden hatte man am Morgen des gleichen Tages 8,9 bis $9,5^{\circ}$ on gefunden. Bei Helsingborg schwankte der Salzgehalt zwischen $11,74^{\circ} \%$ und $33,08^{\prime \prime}{ }_{00}$; eine Sprungschicht lag zwischen 20-23 m. Lappe Grund bekundet für diese Zeit ein ganz ähnliches Bild, der Sprung war hier zwischen $15-20 \mathrm{~m}$.

Ein dagegen von den Fenerschiffsbeubachtungen recht abweichender Fall wurde am 1. Juli 1909 beobachtet. Auf Drogden war am Morgen dieses Tages in $8 \mathrm{~m}$ Tiefe $7,8^{\circ}{ }_{00}$ gefunde.. cin Wert, der auch an den Tagen vorher nicht überschritten wurde. In 10,5 m Tiefe der Flintrinne wurden dagegen $19,56^{\circ}{ }_{00}$ konstatiert. Während sich durch die Drogdenrinne ruhiger Auslauf vollzieht, läuft gleichzeitig salzreiches Wasser über die tiefere Flintschwelle ein. Wir ersehen daraus, daß die Beobachtungen auf dem Feuerschiff Drogden allein nicht maBgrebend sind!

Wie das salzreiche Wasser seinen Weg aus der Kadetinne in die Arkonasee vornimmt, wissen wir nicht. Man fand am 12. Dezember 1908 auf der Dirsser Schwelle in $15 \mathrm{~m}$ Tiefe nur $9,29^{\circ}{ }_{00}$, womit nicht gesagt ist, daß es diesen Rüclien nur stoßweise überschreitet. Es kann sich auch weiter nördlich halten, so traf man am 5. Juni 1908 in der Hjelmbucht südlich Möen salzreiches Bodenwasser.

Ließe sich mittels der Terminfahrten eine Beziehung zwischen dem Salzgehalt am Grund der Kadetrinne zu dem jeweiligen Salzgehalt in $11 \mathrm{~m}$ Tiefe bei Gjedser Rer finden, so wären wir in der Lage, uns über den Verlauf der Salzgehaltsbewegungen am Boden der Kadetrinne eine ungefähre Torstellung zu machen. Leider aber ist es nicht mög- 
lich, wie ich bei der Bildung der Abweichungen zwischen $11 \mathrm{~m}$ Tiefe bei Gieder Rev und 26 in in der Kadetrinne für die regelmäßigen Terminzeiten fand. Am einheitlichsten sind die Abweichungen im Mai, wo sie zwischen $\overline{5}, \bar{\jmath}$ und $9,0^{\circ} \%$ schwanken, am größten im Februar, wo sie sich zwischen 0,7 und $11,3 \%$ bewegen, so daB sie nicht weiter verwertet werden können. Auch zeigt sich kein einheitlicher Zusammenhang zwischen relativ hohem Salzgehalt an beiden Stationen.

Das Bild im Umkreis der Kadetrinne wird durch eine größere Zahl von Serien aus dem .Jahre 1909 vervollständigt. Für den 2. Mai dieses Jahres läßt sich ein Schnitt südlich Falster bis zwei Drittel Weges nach Warnemünde konstruieren. Er zeigt oberflächlich salzreiches Wasser, bis $13,98^{\circ}$ on von Süden aus bis zur Mitte der Strecke, dann eine Abnahme, nördlich $54^{0} 30^{\prime}$ bis auf $8,71^{\circ} \%$. Am Boden vollzieht sich nicht eine ähnliche Verteilung, sondern der höhere Salzgehalt zeigt eine deutliche Abhängigkeit von der Tiefe. Dort, wo sich von Fehmarnbelt kommend der schmalste $\mathrm{Paß}$ zur Kadetrinne einstellt, treffen wir 23,08 " ${ }_{00}$. die beiderseits bei Höhenzunahme des Bodens rasch abnehmen. Eine ähnliche Verteilung zeigen der 20. Mai 1904 und der 17. Mai 1909, letzterer mit dem Hinweis, daß über die niedrige Schwelle im Westen der Kadetrinne 18-19\% salzreiches Wasser häufiger einströmt, jedenfalls nicht nur gelegentlich stoßweise. Daß das Bild sich aber auch gänzlich verändern kann, lehrt ein Schnitt vom 9. Februar 1909, der in der Nähe des $12^{\circ}$ ö. L. wperflächlich das salzreichste Wasser in der Mitte brarłte, am Boden aber eine unregelmäßige Verteilung zeigte.

Für das Gebiet der Mecklenburger Bucht zeigt der Fehmarnbelt in Querschnitten deutlich eine Abnahme des Salzgehaltes von der deutschen Küste aus zur dänischen. Am 7. Februar 1909 hat man $20,12^{\circ}{ }_{00}$ in $12 \mathrm{~m}$ Bodentiefe auf der südlichen Seite gegenüber $19,02 \%$ auf der nördlichen gefunden. Das gleiche Bild begegnet uns in Querschnitten am Ostende der Mecklenburger Bucht wieder (17. April 1909). Die Verteilung in der Tiefe der Bucht weist darauf hin, daß das Einlaufwasser sich bis zum Ausgang der Lübecker Bucht erstreckt, wie der relativ hohe Salzgehalt dartut, den man auf DO 4 so oft gegenüber DO X angetroffen hat. Weitere Züge aufzudecken, sind die Schnitte der Lübecker Bucht zu lückenhaft. Auch für die Oberfläche scheint eine weit größere Zahl ron Beobachtungen notwendig zu sein, um klar zu sehen. Oft zeigt sich, daß auf der mecklenburger Seite der Salzgehalt höher ist als in der Mitte der Bucht, doch kommen auch Ausnahmen vor, wie am 1 Nai 1909. An der Oberfläche scheint das salzreiche Wasser ziemlich in die Lübecker Bucht gedrängt zu werden. 
Für die Verhältnisse im Obergangsgebiet vom Kleinen Belt zum Fehmambelt gestatten uns Beohachtungen, die vom November 1908 bi.s. Juni 1909 ausgeführt wurden, eine Anzahl Serien zu Schnitten zu gruppieren. Einen Srhnitt haben wir in der Talrichtung des Kleinen lieltes gelegt, von der Nordostseite Alsens, wo dicht der Küste zwei Stationen liegen, nach der nördlichen Hälfte der Schwelle Fehmarn-Langeland südlich ihrer Lücko und nach DO 3 im Fehmarnbelt. Das Material gestattet ihn im ganzen zwölfmal zu konstruieren, so daß ein ungefähres Bild über die Wasserverteilung gewonnen werden kann. Auf der Oberfläche zeigt sich nicht immer eine konstante Abnahme des Salzgehaltes nach der Arkonasee zu. DO VI hat wiederholt etwas geringeren Silzqrehalt, im November und Dezember 1908 wie im Mai, August und Oktober 1909. Ebenso hat die nördlichere Station im Alsenbelt wiederholt weniger Salzgehalt als die südlichere, im Januar, März und April 1909 .

Cber die weitere Salzgehaltsverteilung an der Oberfläche unterrichten uns zwei Schnitte, die ich senkrecht zueinander durch die Kieler Bucht gelegt habe. Der eine läuft von der Eckernförder Bucht über Gabelsflach und DO VII nach Fehmarnbelt, bildet also mit dem soeben besprochenen einen spitzen Winkel. Er umfaßt die Stationen DO II, DO 1, DO VII und DO 3 und läßt sich achtmal konstruieren. Der andere läuft von der Hohwachtbucht nach Norden und umspannt die Stationen Hohwachtbucht, DO VII und DO VI und kann dreimal entworfen werden. Der hohe Salzgehalt, den wir auf Station DO VI fanden, setzt sich südwärts bis zur Mitte zwischen Langeland und Wagrien fort. Nur einmal lag an der deutschen Küste das Maximum, am 22. März 1909, in der Regel nimmt der Salzgehalt aber nach Osten hin ab. Zur Eckernförder Bucht steigt er dagegen immer wieder, so daß wir für die Kieler Bucht annehmen dürfen, daß salzreiches Oberflächenwasser sich rom Alsenbelt entlang der Festlandküste an der Ostseite der Kieler Bucht bis zur Bucht von Eckernförde bewegt, dann ostwärts zum Fehmarnbelt nördlich ron Stoller Grund und Gabelsflach. Dieser Auffassung steht das Bedenken gegenüber, daß zwischen dem Südende des Alsenbeltes und der Eckernförder Bucht eine Station DO III gelegen ist, die meistens geringeren Salzgehalt zeigt als DO II in der Eckernförder Bucht. Sie liegt weiter ab rom Lande, so daB sie ron diesem Strom nicht mehr erreicht werden mag, es ist aber auch angängig, daB der Auslauf aus der Flensburger Förde und der Schlei bei ihr den Salzgehalt herabdrückt.

In $20 \mathrm{~m}$ Tiefe ist bei den Schnitten, bei denen alle vier. Stationen vertreten sind, nur einmal in Fehmarnbelt der höchste Salzgehalt ge- 
messen worden, am 21. Mai 1909. Sonst treffen wir ihn immer auf der Fehmarn-Langelandsschwelle, mit Ausnahme vom 15. August 1909, wo er im nördlichen Alsenbelt gelegen ist. Der hohe Salzgehalt bei DO VI deutet darauf hin, daß das durch den Großen Belt einströmende Bodenwasser zunächst seine Richtung nach Süden beibehält, ehe es nach Osten abbiegt. Daß Wasser aus dem Kleinen Belt an der Salzgehaltssteigerung schuld ist, scheint mir dagegen nicht, da die Steigerung gegenüber dem südlichen Alsenbelt stets deutlich ausgeprägt ist. Am Boden kommt die Salzgehaltsabnahme dagegen meistens nicht zum Ausdruck, was nicht verwundern darf, beträgt doch der 'Tiefenunterschied vielfach über $10 \mathrm{~m}$.

Wie weit sich der Einfluß von Wasser aus dem Großen Belt am Boden der Kieler Bucht bemerkbar macht, ist auf Grund des vorliegenden Materials nur schwer zu unterscheiden. Die Hohwachtbucht besitzt auf den zwei Serien, die hierfür in Frage kommen, einen höheren Salzgehalt als die gerade nördlich von ihr gelegene Station DO VII, so daß er nur von Westen gekommen sein kann. Daß dieses indessen nicht immer geschieht, beweisen die Salzgehalte an Boden des Längsschnittes, die kein klares Bild enthüllen. Leider fehlt gerade in der Mitte der Kieler Bucht, etwa auf $10^{\circ} 30^{\prime}$ östl. Länge liegend, eine Station, von der eine größere Anzahl von Beobachtungen zur Verfügung ständen.

Aus dem nördlichen Kleinen Belt liegen nur wenige Tiefenbeobachtungen vor. Am 27. Juli fand man am Boden seines nördlichen Ausganges in $35 \mathrm{~m}$ Tiefe $22,00 \%$, während drei Tage vorher im Großen Belt 32,14\% bei Revsnäs konstatiert wurde. Schnitte können wir für dieses Gebiet nicht konstruieren.

Für die Salzgehaltsverteilung im Großen Belt und Samsöbelt liegen von dänischer Seite nicht außerhalb der Terminfahrten gelegene Stationsbearbeitungen vor, sondern Beobachtungen, die während der Fahrt in je einer Tiefo gewonnen wurden. Es kommen zwei Fahrten in Betracht, vom 21. Januar 1908 zwischen 8 h 52 a. m. bis 12 h 55 p. m. und rom 4. Februar $19099 \mathrm{~h} 46$ a. m. bis 5 . Februar $1 \mathrm{~h} 37 \mathrm{p}$. m.; die letzte war während der Dunkelheit unterbrochen. Beide zeigen oberflächlich eine allmähliche Abnahme des Salzgehaltes. 1908 war er in der Hauptrinne westlich Agersö 25,23\% an der nördlichen Ostseite Langelands treffen wir $24,56 \%$ und $25,91 \%$, und in ähnlicher Höhe bewegt sich der Salzgehalt westlich der Insel nördlich vom Ausgang rom Svendborgsund. 1909 betrug der Salzgehalt auf der Höhe der Kjertemindebucht $23,28 \%$, am Südende Langelands $20,34 \%$. Die Abnahme erfolgt nicht kontinuierlich. 
In der Längstichtung des (iroken Beltes litssen sich verschiedentlich Zunahmen und Abnahmen in kleinem Maßstabe erkennen, deren Ursichen uns verstïndlich werden, wenn wir die Querprofile betrachten. Stellen wir für die strecke zwischen Lolland und Langeland die Gegensätze \%wischen beiden Seiten für ungefähr gleiche Breiten zusammen, so erhalten wir folgendes Bild:

\begin{tabular}{|c|c|c|c|c|c|}
\hline \multicolumn{3}{|c|}{ Westscite } & \multicolumn{3}{|c|}{ Ostseite } \\
\hline $5 t^{0} 5 t^{\prime}, 1$ & $10^{0} 51^{\prime}, 6$ & $20,(151 \%$ & $5.4^{0} 533^{\prime} .8$ & $10^{6} 57^{\prime}, 0$ & $18.62^{\circ} / 00$ \\
\hline $47^{\prime}, 6$ & $49^{\prime}, 5$ & $2(0,5,5)$ & $47^{\prime}, 8$ & $56^{\prime} 3$ & 17,25 \\
\hline $46^{\prime}, 5$ & $48^{\prime}, 2$ & 2()$, 41$ & $46^{\prime}, 5$ & $57^{\prime}, 1$ & 15,52 \\
\hline $45^{\prime}, 5$ & $47^{\prime}, 1$ & 20,44 & $45^{\prime} .3$ & $57^{\prime}, 9$ & 18,03 \\
\hline $44^{\prime}, 2$ & $46^{\prime}, 7$ & 20,34 & $43^{\prime}, 5$ & $58^{\prime}, 9$ & 19,60 \\
\hline $41^{\prime}, 4$ & $50^{\prime}, 8$ & 21,69 & $41^{\prime}, 4$ & $11^{\circ} 00^{\prime}, 1$ & 20,37 \\
\hline
\end{tabular}

Die Tabelle läßt ein salzhaltiges Einlaufwasser an der Westseite, ein salzärmeres Auslaufwasser an der Ostseite ron diesem Stück des Großen Beltes erkennen. Die Trennung ist nicht etwa eine Folge ron geringen Zeitunterschieden in den Beobachtungen, sondern eine örtliche. Denn als der Dampfer, der zuerst in Nord-Südrichtung an der Westseite gefahren war, dann sich auf der Höhe des nördlichen Langeland von neuem der westlichen Seite zuwandte, traf er die gleichen Verhältnisse wie am Anfange seiner Beobachtungen wieder an. Ferner ist aus der Tabelle eine ungleichmäßige Abnahme des Salzgehaltes zu erkennen, namentlich an der Ostseite. Sie wird daher rühren, daß die Positionen nicht im gleichen Abstande ron der Küste gelegen sind.

Dieses allgemeine Bild wird des mehrfachen bestätigt durch Querschnitte, braucht aber nicht immer einzutreten. Die Abweichungen schwanken aber nur um geringe Beträge. Daß sich das Wasser dabei nicht nur seitlich nebeneinander bewegt, sondern auch übereinander, zeigen die rerschiedenen Strombeobachtungen, die im Großen Belt ausgeführt wurden. So maß man am 17. Norember 1908 im Langelandsbelt bei $15 \mathrm{~m}$ Tiefe aus Süden kommenden Strom. in $42 \mathrm{~m}$ Tiefe aus Norden laufenden. Daß die Bewegungen aber auch komplizierter werden können, lehren dänische Strommessungen vom 5. Mai 1910 in der Westrinne bei Sprogö. Man fand beispielsweise, am Nachmittag $5 \mathrm{~h}$ in $2,5 \mathrm{~m} \mathrm{~S} 33 \mathrm{E}$, in $5 \mathrm{~m} \mathrm{~S} 33 \mathrm{E}$, in $10 \mathrm{~m} \mathrm{~S} 11 \mathrm{~W}$, in $15 \mathrm{~m} \mathrm{~N} 57 \mathrm{E}$, in $20 \mathrm{~m} \mathrm{~S} 12 \mathrm{~W}$, in $25 \mathrm{~m} \mathrm{~S} 12 \mathrm{~W}$. Bis $10 \mathrm{~m}$ Tiefe war der Strom kräftig, dann wurde er schwächer.

Die Fortsetzung des Auslaufes ins Kattegat möge den BeschluB dieser Einzelbilder bringen. Ihre Darstellung wird durch eine Novemberfahrt von 1907 ermöglicht. Während des 2. November wird überall 
auf der Oberfläche des Großen Beltes ein Salzgehalt unter $12 \%$ angetroffen. Zwölf Stationen weit vom Nordende an bis wenige Kilometer nördlich Sprogö schwanken die Werte zwischen 12 und $11^{\%} /{ }_{00}$, in der Nähe dieser Insel zwischen 11 und $10,50 \%$. Das süßere Wasser ist nur eine dünne Lage, die nach Süden an Mächtigkeit zunimmt. So wird zwischen $55^{0} 36^{\prime}, 5$ und $36^{\prime}, 8$ bei 0 Meter $11,42^{\circ} \%$ gemessen, bei $7,7 \mathrm{~m} \mathrm{23,10^{ \circ }}$ on mit regelmäBiger Tieferlegung des Sprunges, zwischen $55^{0} 28^{\prime}, 6$ und $55^{0} 28^{\prime}, 2$ bei $0 \mathrm{~m} 11,80^{\circ} \%$, bei $8 \mathrm{~m} 12,72^{\circ} \%$, bei $13 \mathrm{~m}$ $26,13 \%$. In größeren Tiefen herrscht überall der normale hohe Salzgehalt. Nach Süden hin können wir die Verhältnisse nicht weiter verfolgen, da die Fahrt bei Sprogö abbricht. Sie erstreckte sich vorher über den ganzen östlichen Teil des südlich Anholt gelegenen Kattegat, begann am 1. November 1907 bei Kullen, bewegte sich zuerst nordwärts, dann nordnordwestlich über den Kleinen Mittelgrund. Die Rückfahrt ging über Anholt Knob und Hesselö in den Samsöbelt. Die Verteilung des Salzgehaltes an der Oberfläche dieses Gebietes gibt über den Weg des Auslaufwassers keinen Fingerzeig auf Grund dieser Fahrt, mag es aus dem Sund oder aus dem Großen Belt stammen. Auch die Mächtigkeit der Oberschicht läßt keinen Schluß zu. Ihre untere Grenze habe ich, da keine Beobachtungen von Meter zu Meter vorliegen, durch rechnerische Mittelbildung aus der letzten Tiefe, in der noch Auslaufwasser angetroffen wurde, und der ersten, in der Einlauf war, gewonnen. Durch dieses angenäherte Verfahren ergab sich, daß von Kullen bis zum Großen Mittelgrund die Mächtigkeit des Auslaufes allmählich von $7 \mathrm{~m}$ auf $22 \mathrm{~m}$ stieg, dann folgte nördlich des Sattels, der sich von Anholt gegen Osten erstreckt, über $14 \mathrm{~m}$ eine Abnahme bis auf $10 \mathrm{~m}$ Tiefe, die zunächst beibehalten wird, um bis $57^{\circ} 29^{\prime}$ auf $15,5 \mathrm{~m}$ zu steigen. Hier bog der Kurs des Schiffes nach Süden, wo man nunmehr bis nördlich Anholt wieder eine geringer werdende Mächtigkeit, bis zu 7,5 m antraf. Südlich Anholt setzt dann von neuem eine unvermittelte Zunahme auf $11 \mathrm{~m}$ ein, die allmählich bis zum Nordrande des Großen Beltes auf 7-8 $\mathrm{m}$ geht. Hier ist dann eine zwischen 10,5 $\mathrm{m}$ und $14 \mathrm{~m}$ schwankende Mächtigkeit gefunden, die scheinbar nicht an die Tiefenverhältnisse geknüpft ist.

Das Gesamtbild, das diese Fahrt zeitigt, ist eine Decke relativ süßen Wassers, die vom Nordende des Sundes und Großen Beltes an Mächtigkeit zunimmt, um auf der Schwelle im Osten Anholts auffallend abzunehmen und erst weiter nordwärts wieder zuzunehmen. Wir dürfen wohl kaum annehmen, daß diese eine Fahrt die normalen Verhältnisse wiederspiegelt. 
Tahelle 8. Synoptische schnitte für den salzgehalt in "

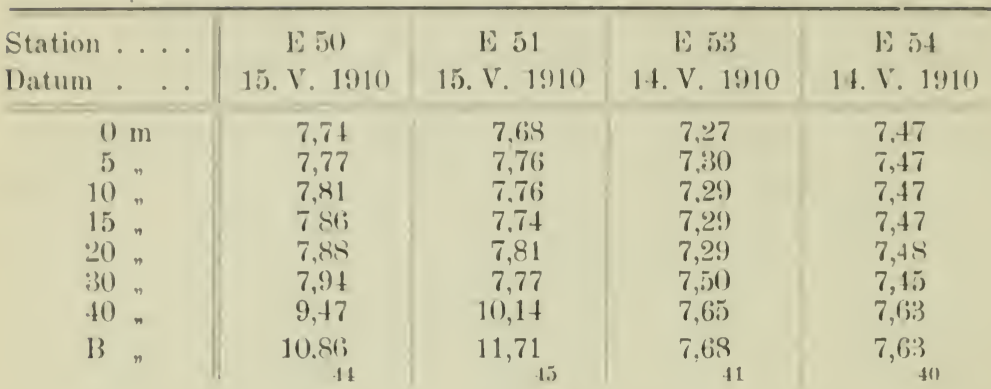

\begin{tabular}{|c|c|c|c|c|c|c|}
\hline $\begin{array}{l}\text { Station } \\
\text { Datum }\end{array}$ & $\cdots$ & $\begin{array}{c}\text { E } 49 \\
\text { 18. VII. } 1907\end{array}$ & $\begin{array}{c}\text { E } 48 \\
\text { 18. VII. } 1907\end{array}$ & $\begin{array}{l}\text { E } 47 \\
\text { 18. VII. } 1907\end{array}$ & $\begin{array}{c}\text { E } 46 \\
\text { 18. VII. } 1 \subseteq 07\end{array}$ & $\begin{array}{l}\text { E } 45 \\
\text { 17. VII. } 190\end{array}$ \\
\hline $\begin{array}{r}0 \\
5 \\
10 \\
15 \\
20 \\
\mathrm{P}\end{array}$ & $\begin{array}{l}\text { m } \\
" \\
" \\
"\end{array}$ & $\begin{array}{l}7,97 \\
7,97 \\
7,99 \\
7,99 \\
-\end{array}$ & $\begin{array}{l}7,63 \\
7.63 \\
7,63 \\
7.70 \\
7,72\end{array}$ & $\begin{array}{l}7,47 \\
7,47 \\
7.47 \\
7,63\end{array}$ & $\begin{array}{l}7,48 \\
7,50 \\
7.50 \\
8.10 \\
-\end{array}$ & $\begin{array}{r}7,77 \\
7,79 \\
7,90 \\
10.59 \\
13,91 \\
13.91\end{array}$ \\
\hline & $\eta$ & 6.99 & $\begin{array}{r}1,88 \\
22,2\end{array}$ & 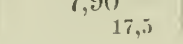 & 0.2 & \\
\hline
\end{tabular}

\begin{tabular}{|c|c|c|c|c|}
\hline $\begin{array}{l}\text { Station . } \\
\text { Datum . }\end{array}$ & $\begin{array}{l}\cdots \\
\ldots\end{array}$ & $\begin{array}{c}\text { DO IS } \\
\text { 17. VIII. } 1909\end{array}$ & $\begin{array}{c}\text { DOF } \\
\text { 17. VIII. } 1909\end{array}$ & $\begin{array}{c}\text { D O VIII } \\
\text { 17. VIII. } 1909\end{array}$ \\
\hline $\begin{array}{r}0 \mathrm{~m} \\
5 \\
10 \\
15 \\
20 "\end{array}$ & $\begin{array}{l}\mathrm{m} \\
" \\
" \\
"\end{array}$ & $\begin{array}{l}11,15 \\
11,11 \\
13,08 \\
18,22 \\
19,61\end{array}$ & $\begin{array}{l}13,33 \\
13,28 \\
15,07 \\
16,87 \\
17,90\end{array}$ & $\begin{array}{l}12,83 \\
13,09 \\
13,35 \\
15.21 \\
18,57\end{array}$ \\
\hline B, & $"$ & 20,17 & $\begin{array}{r}18,8 t \\
25\end{array}$ & $\begin{array}{r}21,69 \\
23\end{array}$ \\
\hline
\end{tabular}

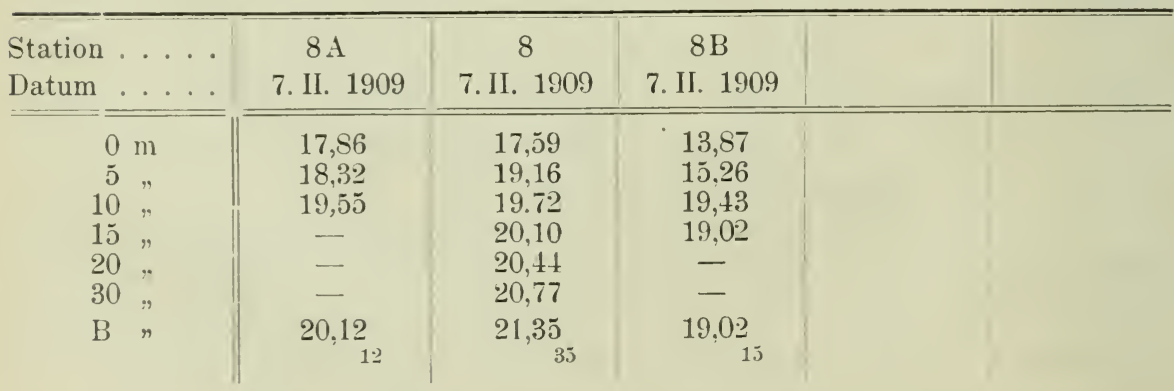

Die untenstehende kleinere Zahl bei $\mathrm{B}$ gibt die jeweilige Bodentiefe an. 


\begin{tabular}{|c|c|c|c|c|c|c|c|}
\hline $\begin{array}{l}\text { Station } \\
\text { Datum }\end{array}$ & & $\begin{array}{c}54^{0} 15^{\prime} \\
12^{0} 0^{\prime} \\
17 . I V .1909\end{array}$ & $\begin{array}{c}5 t^{0} \quad 19^{\prime} \\
12^{0} 03^{\prime} \\
\text { 17. IV. } 1909\end{array}$ & $\begin{array}{c}54^{0} \quad 2.3^{\prime} \\
\left.11^{0} \quad 5 !\right)^{\prime} \\
\text { 17. IV. } 1909\end{array}$ & $\begin{array}{c}54^{0} .28^{\prime} \\
11^{0} 55^{\prime} \\
\text { 17. IV. } 1909\end{array}$ & $\begin{array}{c}54^{0} 29^{\prime} \\
11^{0} 5^{\prime} \\
\text { 17. IV. } 1909\end{array}$ & $\begin{array}{r}54^{0} 32^{\prime} \\
11^{0} 55^{\prime} \\
17 . \text { IV. } 1909\end{array}$ \\
\hline $\begin{array}{r}0 \\
5 \\
10 \\
15 \\
20 \\
\text { B }\end{array}$ & $\begin{array}{l}m \\
" \\
" \\
"\end{array}$ & $\begin{array}{c}11, \times 9 \\
- \\
- \\
- \\
- \\
12,21 \\
7\end{array}$ & $\begin{array}{c}10,52 \\
10,02 \\
11,76 \\
16,71 \\
18,24 \\
19\end{array}$ & $\begin{array}{l}10,28 \\
10,32 \\
11,26 \\
17,7 \cdot 2 \\
- \\
18,06 \\
17,5\end{array}$ & $\begin{array}{r}8,50 \\
8.59 \\
9,96 \\
17,59 \\
22,56 \\
23,50 \\
21\end{array}$ & $\begin{array}{c}8,50 \\
8,68 \\
8.87 \\
- \\
- \\
15 \check{05}\end{array}$ & $\begin{array}{c}8.33 \\
8.47 \\
- \\
- \\
- \\
8,51 \\
6,5\end{array}$ \\
\hline
\end{tabular}

\begin{tabular}{|c|c|c|c|c|c|c|c|c|}
\hline $\begin{array}{l}\text { Station. . } \\
\text { Datum . }\end{array}$ & $\begin{array}{c}0 \mathrm{XI} \\
\text { 1S. XII. } \\
190 \mathrm{~S}\end{array}$ & $\begin{array}{c}0 \mathrm{X} \\
\text { 16. XII. } \\
1908\end{array}$ & $\begin{array}{c}4 \\
\text { 18. XII. } \\
1908\end{array}$ & $\begin{array}{c}\text { Lüb. I3 } \\
\text { 18. XII. } \\
1.908\end{array}$ & $\begin{array}{c}0 \mathrm{XI} \\
16 . \mathrm{II}^{\circ} \\
1909\end{array}$ & $\begin{array}{r}\text { OX } \\
16 . \mathrm{IV} . \\
1909\end{array}$ & $\begin{array}{c}4 \\
16 \mathrm{IV} . \\
1909\end{array}$ & Lüb. 1). \\
\hline $0 \mathrm{~m}$ & 12,23 & 11.13 & 17.21 & 17,97 & $10.1 t$ & 11.47 & 13,15 & - \\
\hline & 12.72 & 13,86 & 17,21 & 18.89 & 10.23 & 1162 & 13,17 & $\ldots$ \\
\hline 10 & 14,60 & 16,59 & 17,63 & 19,61 & 13.51 & 13,06 & 13.19 & - \\
\hline 15 & 16,28 & - & 18,68 & 20,90 & 15,41 & 13,60 & 14.79 & .. \\
\hline 20 & $2 \cdot 2,34$ & - & 2126 & - & 17,97 & 17,99 & 18,70 & - \\
\hline $30 "$ & - & - & - & & - & - & & - \\
\hline B , & $\underset{20}{22,34}$ & $\begin{array}{r}17,14 \\
11\end{array}$ & 21.79 & 21,24 & 17.97 & 2 & 20,34 & - \\
\hline
\end{tabular}

Datum .. 22 I. 09

22. I. 09

1. V.09 1. V.09 1. V. 09

\begin{tabular}{r|c|c|c|c|c|c|c|c}
\hline $0 \mathrm{~m}$ & 15,95 & - & 16,04 & - & 12,45 & 11,42 & 10,05 & - \\
5 & 16,53 & - & 17,47 & - & 12,63 & & 10,12 & - \\
$10 "$ & 17,12 & - & 18,03 & - & 12.81 & & 14,54 & - \\
$15 "$ & 17,41 & - & 18,26 & - & 12,99 & 17,00 & 17,68 & - \\
$20 "$ & 17,70 & - & 19,87 & - & 14,96 & 19,91 & 21,41 & - \\
$30 "$ & - & - & - & - & - & - & - & - \\
B " & 17,76 & - & 20,19 & - & 14,96 & 22,23 & 22,16 & - \\
& 21 & & 21 & & 20 & 24 & 21 &
\end{tabular}

\begin{tabular}{l|l|l|l|l|l|l|l|l|l|l|l|l} 
Datum .. & 8. II. 09 & 8. II. 09 & 8. II. 09 & 8. II. 09 & 21.V. 09 & 21.V. 09 & 21.V. 09
\end{tabular}

\begin{tabular}{|c|c|c|c|c|c|c|c|}
\hline $0 \mathrm{~m}$ & 14,33 & 13,62 & 14,96 & 15.14 & 13,12 & 12.74 & 13,15̃ \\
\hline $5 \%$ & 15,05 & - & 15.05 & 14.90 & 13,30 & 13,66 & 13,12 \\
\hline 10 & 17,29 & - & $1 \check{0}, 43$ & 15,84 & 13,48 & 12,99 & 13.21 \\
\hline & 18,04 & 一 & 18,33 & 18,24 & $13,89)$ & 13,06 & 13,26 \\
\hline 20 & 19.29 & - & 20,17 & 20,16 & 20,06 & 16,42 & 20,99 \\
\hline 30 " & - & - & - & - & - & - & - \\
\hline$B$ n & $\begin{array}{c}19,29 \\
23,5\end{array}$ & $\begin{array}{r}18,98 \\
25\end{array}$ & 20,91 & $\begin{array}{r}20,55 \\
21\end{array}$ & $23,01_{21}$ & $\begin{array}{r}24.94 \\
25,5\end{array}$ & 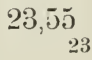 \\
\hline
\end{tabular}

Datum .. 25. III.09

23.III. 09 24.III. 09 3. VI. 09

3. VI 09

\begin{tabular}{|c|c|c|c|c|c|c|c|c|}
\hline $0 \mathrm{~m}$ & 9,51 & - & 11,22 & 12.59 & $9,1 \tilde{5}$ & - & 11,46 & - \\
\hline $5 \%$ & 11,0 Ј & $\ldots$ & 12,58 & 13,33 & 9,15 & - & 11.46 & - \\
\hline 10 & 13,41 & - & 14,29 & 14,39 & 11,96 & - & 12.72 & - \\
\hline $15 "$ & $15,8 t$ & - & 15,53 & 15.71 & 15,28 & 一 & 14,40 & - \\
\hline $20 "$ & - & - & 18,91 & 16.35 & - & - & 21,46 & - \\
\hline $30 n$ & - & - & - & - & - & - & - & - \\
\hline B " & $\begin{array}{r}16,09 \\
17\end{array}$ & - & $18,66_{24}$ & $16,3 \check{2}$ & $\begin{array}{r}20,64 \\
19\end{array}$ & - & 21,46 & - \\
\hline
\end{tabular}

Die untenstehende kleinere Zahl bei $\mathrm{B}$ gibt die jeweilige Bodentiefe an. 


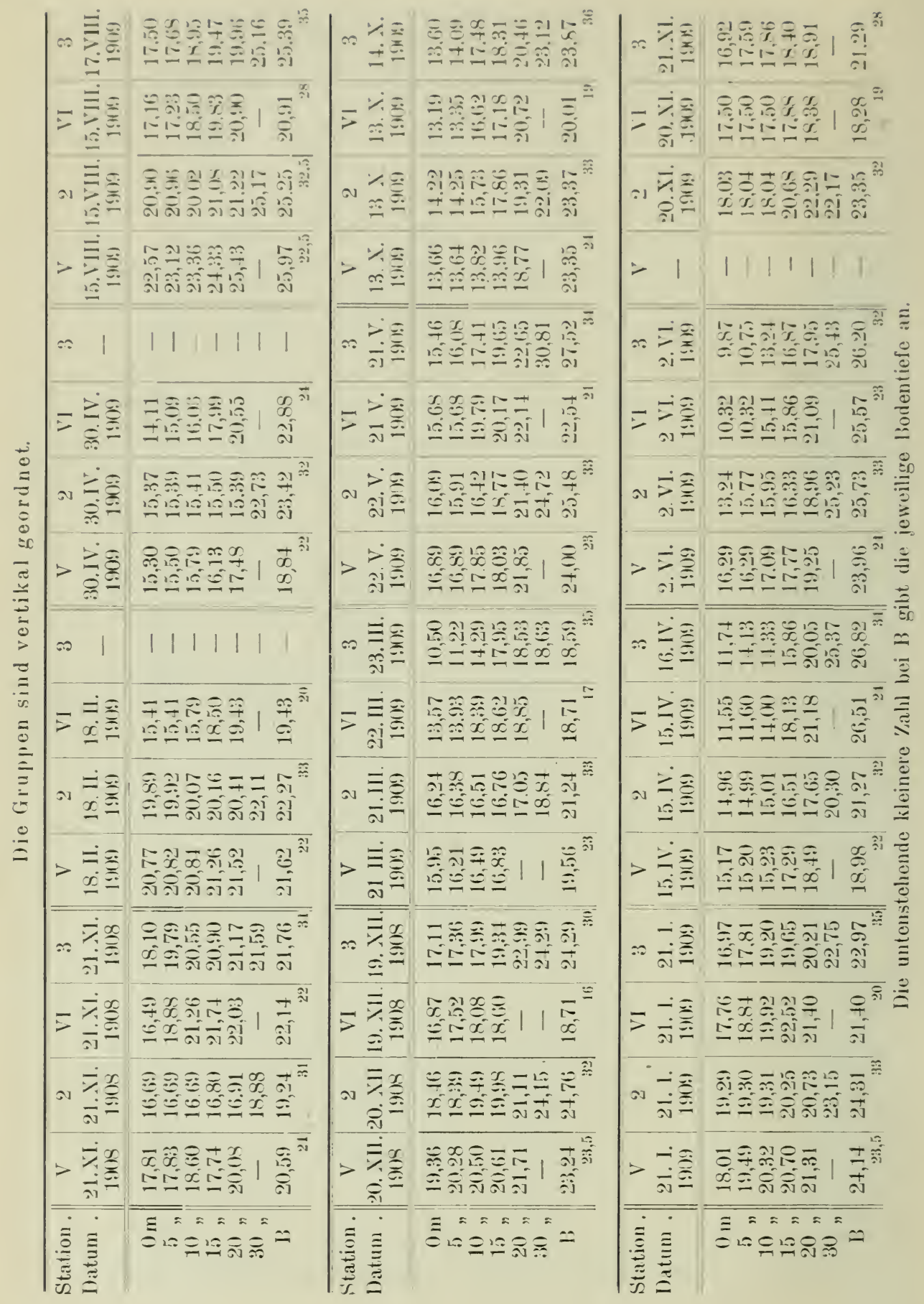




\begin{tabular}{|c|c|c|c|c|c|c|}
\hline & 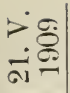 & 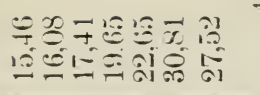 & $\therefore$ 三咅 & 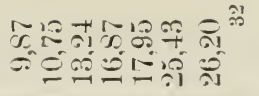 & & \\
\hline 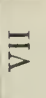 & ஸे & 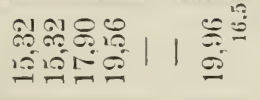 & $\equiv 1$ & 1111 & & \\
\hline- & 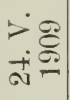 & 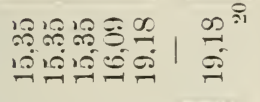 & 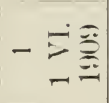 & 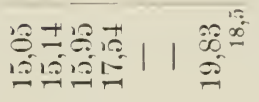 & & \\
\hline$=$ & $\begin{array}{l}>0 \\
\dot{\sigma} \\
\dot{\sigma}\end{array}$ & 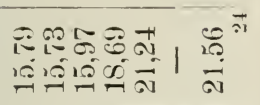 & 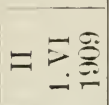 & 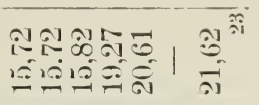 & & \\
\hline$\infty$ & $=\dot{g}$ & 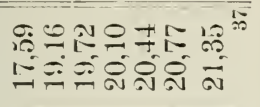 & 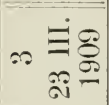 & 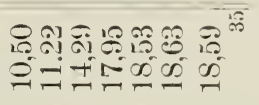 & $\infty$ 己 & 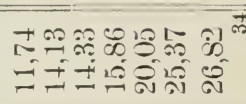 \\
\hline 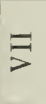 & $\begin{array}{l}\exists \\
\stackrel{g}{g}\end{array}$ & 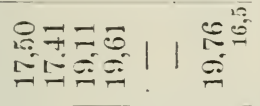 & 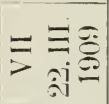 & 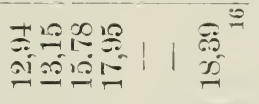 & $\sqsupseteq$ & 1111 \\
\hline- & 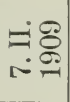 & 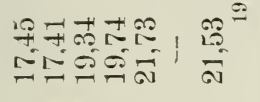 & 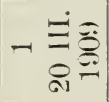 & 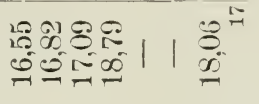 & 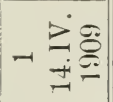 & 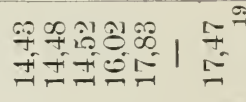 \\
\hline$\exists$ & $\begin{array}{l}-\dot{g} \\
\omega \\
\dot{0}\end{array}$ & 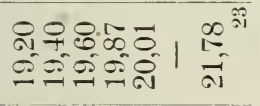 & 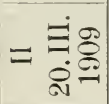 & 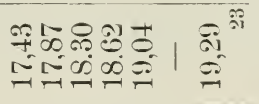 & 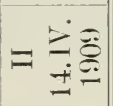 & 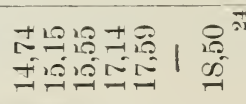 \\
\hline$\infty$ & 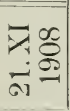 & 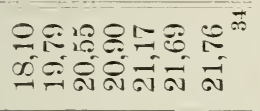 & 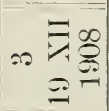 & 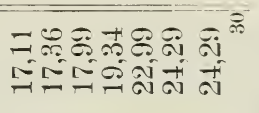 & 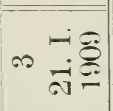 & 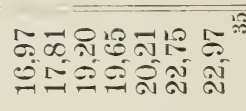 \\
\hline$=$ & 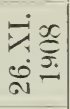 & 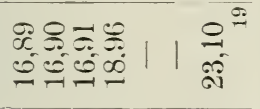 & 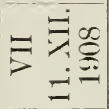 & 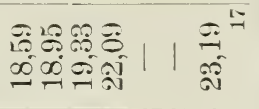 & $\equiv$ & 111 \\
\hline$\neg$ & $\begin{array}{l}-\infty \\
\dot{0} \\
\dot{0}\end{array}$ & 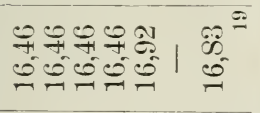 & 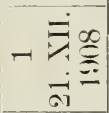 & 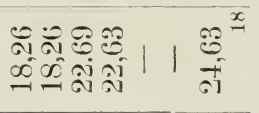 & - $\dot{0} \dot{0}$ & 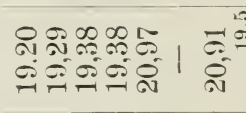 \\
\hline 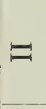 & 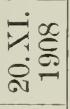 & 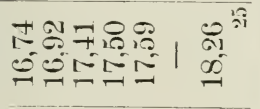 & 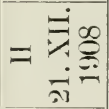 & 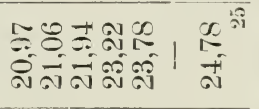 & $=\frac{-\dot{\delta}}{\dot{\Omega}}$ & 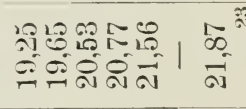 \\
\hline & & 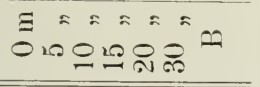 & & 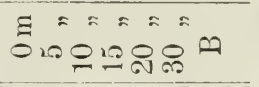 & & 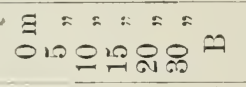 \\
\hline$\frac{3}{0}$ & $\begin{array}{l}\exists \stackrel{\infty}{\Xi} \underset{=}{=} \\
=\end{array}$ & 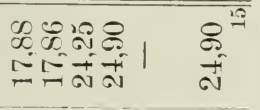 & 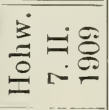 & $\stackrel{0}{=}|1| \sigma^{\circ}$ & 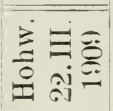 & 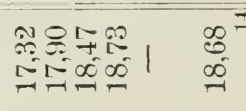 \\
\hline 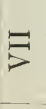 & 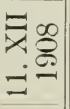 & 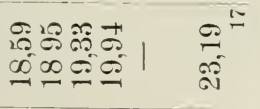 & 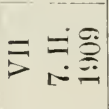 & 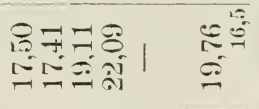 & 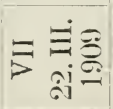 & 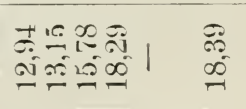 \\
\hline 5 & 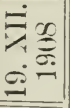 & 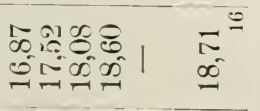 & $\Rightarrow \doteq \tilde{\Xi}$ & 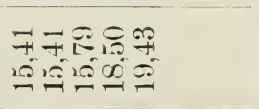 & 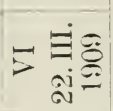 & 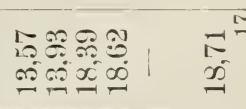 \\
\hline 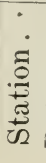 & 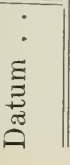 & 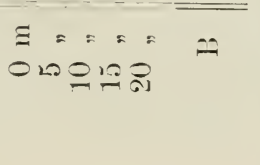 & 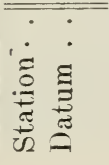 & $\begin{array}{l}\Xi=2= \\
=12=100\end{array}$ & 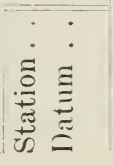 & $\begin{array}{l}\Xi==== \\
0100108\end{array}$ \\
\hline
\end{tabular}


Verzeichnis der Synonyma der Stationbezeichnungen.

\begin{tabular}{|c|c|c|}
\hline $\begin{array}{c}\text { Lis August } 1906 \text {; } \\
110 \text { stseo }\end{array}$ & $\begin{array}{c}\text { Bis August } 1907 \\
\text { D Beltsee }\end{array}$ & $\begin{array}{c}\text { Seit August } 1907 \\
110\end{array}$ \\
\hline 1 & I & I \\
\hline 2 & $\mathrm{IV}^{\mathrm{T}}$ & IV \\
\hline 3 & V'III & VII \\
\hline 4 & IX & VIII \\
\hline 5 & XIII & $\mathrm{I}$ \\
\hline 6 & 6 & 6 \\
\hline 7 & 7 & 7 \\
\hline 8 & \& & 8 \\
\hline !) & 9 & () \\
\hline 10 & & XIII \\
\hline
\end{tabular}

Der Karte S. T2 sind die bis 1906 verwandten Bezeichnungen zugrunde gelegt. Die mit römischen Ziffern eingetragenen Stationen sind die späteren Ergänzungsstationen. Man beachte den Unterschied zwischen DOX der Karte und dem Synonym DO5 = DOX.

\section{Zukünftige Aufgaben.}

In dem Cbergangsgebiet der Beltsee und des Sundes bekundet der Salzgehalt die schärfsten Gegensätze in der Zusammensetzung des Wassers. Mit ihm haben wir deshalb die ozeanographische Betrachtung der șüwestlichen Ostsee eröffnet.

Er hängt in hohem Maße von Einlauf und Auslauf ab. Stürme aus SSW bis ENE bescheren Einlauf, solche aus NNE bis NNW Auslauf. Bei weniger heftigen Luftbewegungen kommt der gleiche Einfluß des Windes nicht so scharf zum Ausdruck. Aus der Verteilung ron Einlauf und Auslauf und ihren Stromgeschwindigkeiten läßt sich für die Beltsee berechnen, daß das jährliche Einlaufquantum rund $350 \mathrm{ckm}$ ausmacht, das Auslaufquantum $850 \mathrm{ckm}$. Von diesen Wassermengen bewegen sich beim Einlauf etwa $220 \mathrm{ckm}$ durch den Stammbelt und $125 \mathrm{ckm}$ durch den Sund. Am Auslauf ist der Stammbelt mit $210 \mathrm{ckm}$ und der Sund mit 640 clim beteiligt.

Die monatlichen Differenzen zwischen Auslauf und Einlauf kommen in den Monatsmitteln des Salzgehaltes der Oberfläche zum Ausdruck, während sie mit denen der Tiefe nicht immer harmonieren. Eine intensive, häufig eintretende Durchmischung stört jedoch das gesamte Bild, so daß das in die Arkonasee einlaufende Wasser keineswegs aus salz- 
reichem Bodenwasser besteht, sondern aus einer Mischung von Auslauf und Einlauf. Das gleiche gilt für das Auslaufwasser.

Die Salzgehaltsextreme des Bodens wandern rom Kattegat in die Gotlandsee hinein. Die große Herbstanschwellung des Atlantischen Wassers macht sich im Winter in der Arkonasee bemerkbar. Die Schwankungen an der Oberfläche wandern hingegen aus der Gotlandsee hinaus. Zwischen beiden schaltet sich in der Regel keine Zwischenschicht ein.

Das sind einige der wichtigsten Züge, die uns der Salzgehalt enthüllt. Wir müssen nunmehr daran gehen, sie zu prüfen und zu erweitern durch eine Auswertung der Temperaturbeobachtungen, was im nächsten Abschnitt der vorliegenden Studien geschehen soll. Ehe wir ihn eröffnen, wollen wir jedoch kurz darlegen, welche Aufgaben uns zur Erweiterung des Bildes vom Salzgehalte besonders wichtig erscheinen. Natürlich drängt sich eine Fülle ozeanographischer Wünsche zur Klärung von Fragen auf, wir wollen aber nur jene herausgreifen, die sich mit verhältnismäßig geringen Unkosten im Anschluß an die jetzt bestehenden ozeanographischen Unternehmungen ausführen lassen.

Zunächst sei eine Gruppe von Forschungen genannt, deren-Vornahme sowohl auf den Feuerschiffen wie bei den Fahrten der Internationalen Meeresforschung dringend erwünscht erscheint. Bei der Beurteilung der großen Wasserbewegungen der Ostsee ist eine möglichst gute Kenntnis ihres Wasserhaushaltes von eminenter Bedeutung. Eine solche läßt sich aber nur dann erreichen, wenn die bis jetzt in ihrer Größe unsicheren Faktoren des Niederschlages und der Verdunstung auf dem freien Wasser einigermaßen scharf gefaßt werden können. Dazu ist erforderlich, daß Verdunstungs- und Regenmessungen vorgenommen werden. Die Verfahren der Verdunstungsmessung sind in den letzten Jahren mehrfach praktisch erprobt worden. Die günstige Aufstellung von Regenmessern wird zunächst mit einigen Schwierigkeiten zu kämpfen haben, die sich auch bei kardanischem Aufhängen im Maste nicht ganz beseitigen lassen. Immerhin dürfte aber hier die Durchführung in der Wirklichkeit besser zum Ziele leiten als theoretische Erwägungen über die Ausführbarkeit.

Als eine dringende Aufgabe der Internationalen Meeresforschung sehe ich es an, die langjährigen Untersuchungen auf den dänischen Feuerschiffen so zu ergänzen, daß es möglich ist, sie in wichtigen Fragen, für die sie ein einzig dastehendes Material liefern, auszuwerten. Es handelt sich namentlich um die Kenntnis der hydrographischen Position der 
Fenerschiffo zu ihrer Lmgebung; insonderheit zu dem Querschnitt der Wasserstraßen im Stammbelt und im südlichen Sund. Die Notwendigkeit, eine solche Kenntnis zu erlangen, zeigen die wenigen Beobachtungen in der Flintrinne. Es würde genügen, daß bei typischen Wetterlagen Ergänzungsbeobachtungen in den Querschnitten angestellt werden, wio ich es schon auf S. 23 erwähnte.

Wenn weitere Fahrten unternommen werden, so wäre eine Station in der Mitte der Kileler Bucht, wie schon bemerkt, recht willkommen; dringend erforderlich sind solche südlich der Kadetrinne nach der deutschen Küste hin wie in den tiefsten Fläehen nördlich der Möenbank in dem Schnitt Arkona-Schonen. DO6 liegt hier schon zu weit nördlich. Besondere Bedeutung würde ferner Fahrten in der Arkonasee Ende Dezember oder Anfang Januar zum Studium des atlantischen Wassers zukommen. Sei es nun, daß solchen Aufgaben künftighin bei den Fahrten der Internationalen Meeresforschung nachgegangen wird, sei es, daß die Fahrten sich in den bisherigen Bahnen bewegen, für unumgänglich notwendig erachte ich es wegen der sich rasch ändernden Wasserbewegungen in der südwestlichen Ostsee, daß die Zeiten, während der die Beobachtungen auf den einzelnen Stationen ausgeführt sind, ständig veröffentlicht werden. Es ist mir wiederholt, namentlich bei der Station in der Kadetrinne und dem Feuerschiff Gjedser Rev, nicht möglich gewesen, die Beobachtungen der Internationalen Meeresforschung mit jenen auf den Feuerschiffen in Beziehung zu setzen, weil die Stundenangaben im Bulletin der Internationalen Meeresforschung fehlten!

Hinsichtlich der Feuerschiffe wäre es im höchsten Grade wünschenswert, daB die Beobachtungen auf Fehmarnbelt nach den gleichen Gesichtspunkten erfolgen wie auf den dänischen Feuerschiffen, namentlich hinsichtlich der Zeiteinteilung und der Kontrolle durch Titrieren. Die Untersuchungen, die Carp hier vorgenommen hat, ${ }^{1}$ ) lassen das Verlangen nach gelegentlichen vielstündigen Beobachtungen wach werden; dankbar muß es anerkannt werden, daß solche in der neuesten Zeit auf dänischen Feuerschiffen ausgeführt sind. $\left.{ }^{2}\right)$

$\left.{ }^{1}\right)$ O. Carp, Der Fehmarnbelt im August und September 1909. Dissertation. Berlin 1912.

2) Vgl. J. P. Jacobsen, Beitrag zur Hỵdrographie der dänischen Gewāsser. Medd. fra Kommissionen for Harundersögelse, Serie Hỵdrografi, Bd. II, Ar. 2. Kopenhagen 1913. 


GC

274

564

Bd.I
Spethmann, Hans Studien zur Ozeanographie der südwestlichen Ostsee

PLEASE DO NOT REMOVE CARDS OR SLIPS FROM THIS POCKET

UNIVERSITY OF TORONTO LIBRARY 


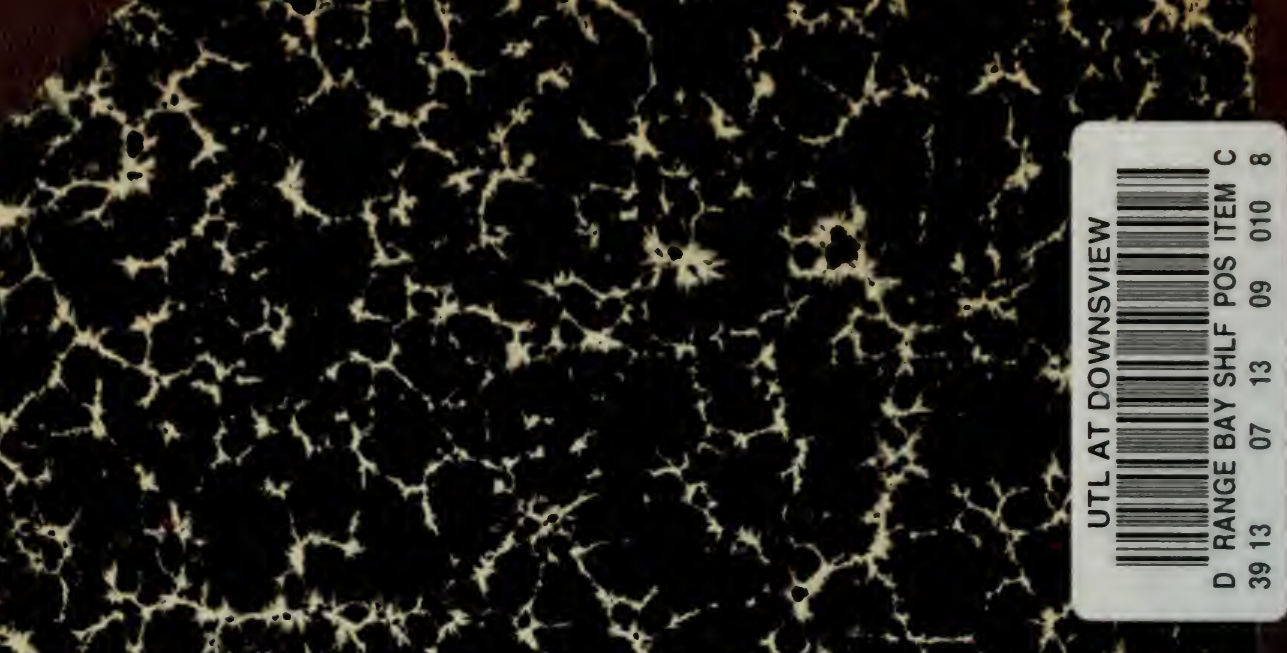
$x^{2}+10 x$

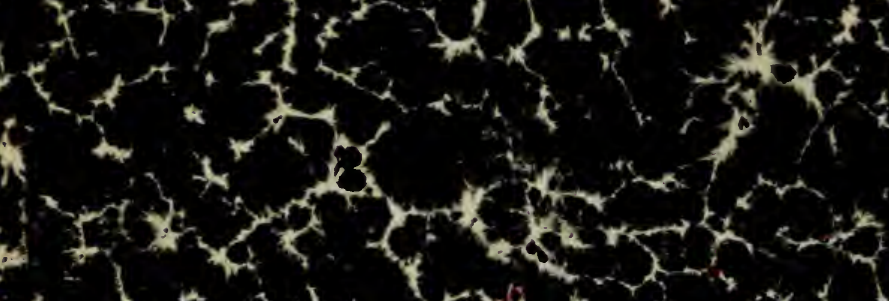

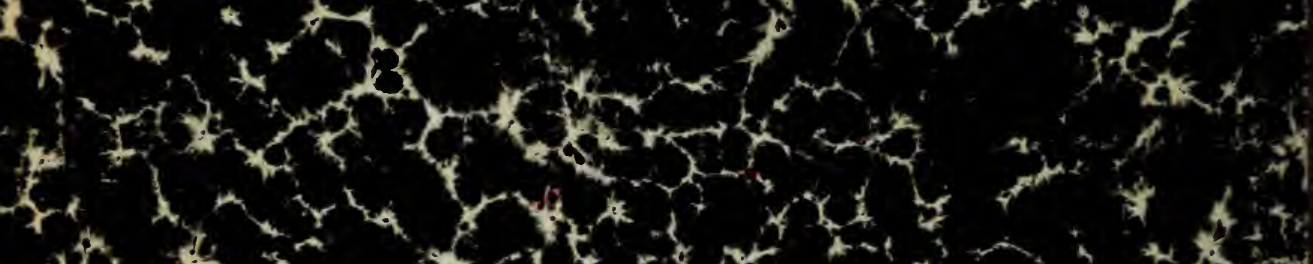

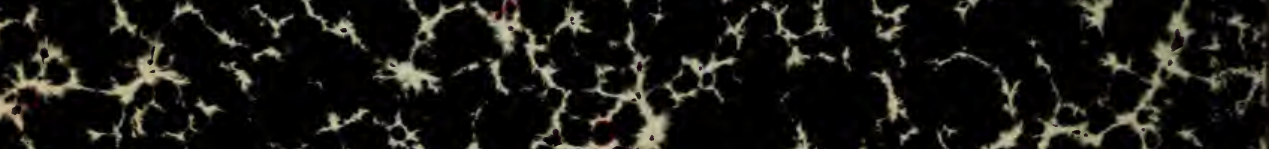

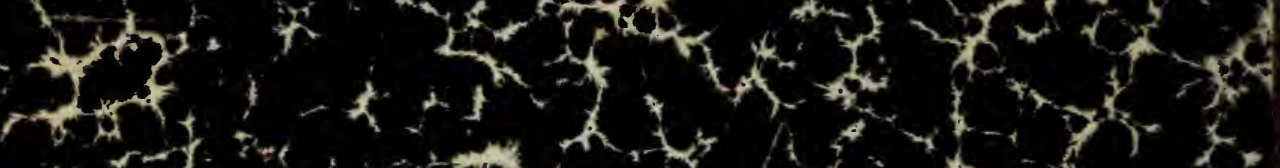

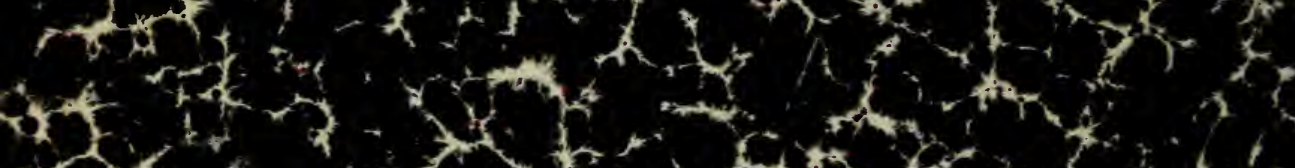

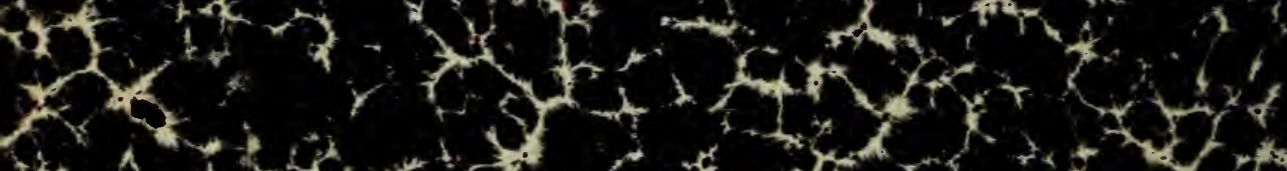
( $x^{2}+x^{2}-x^{2}+x^{x}$

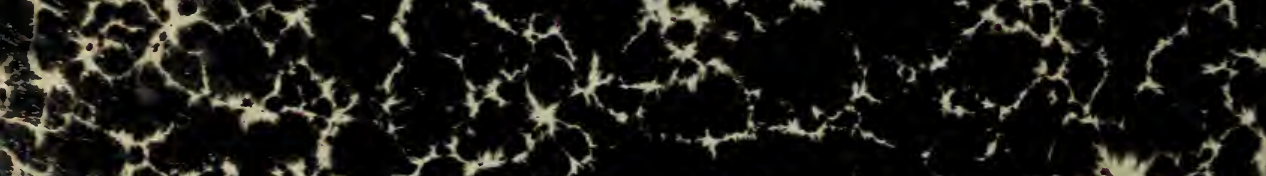

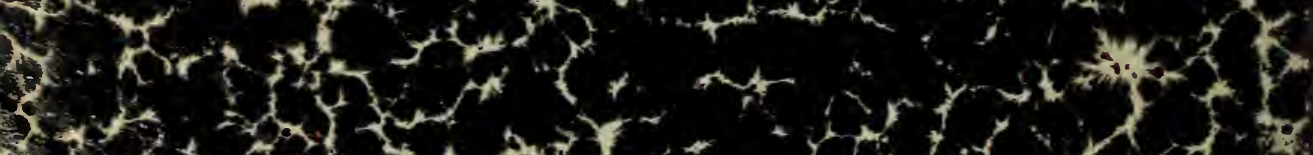

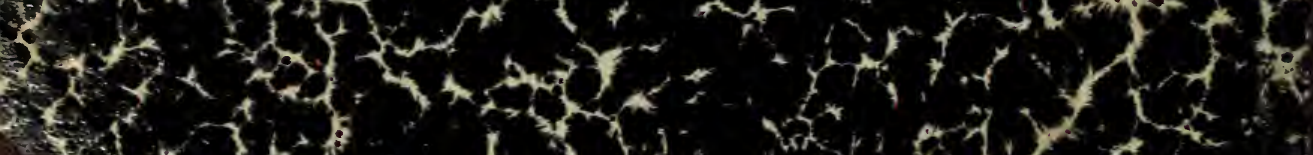

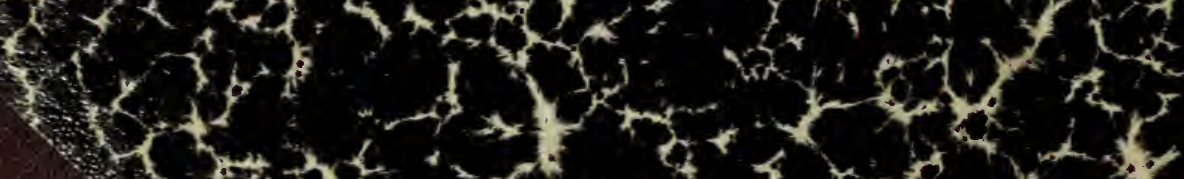
$4+3(-2+2+2$ 